\title{
WestVirginiaUniversity
}

THE RESEARCH REPOSITORY @ WVU

Graduate Theses, Dissertations, and Problem Reports

2015

\section{Plasmonic Enhancement Mechanisms in Solar Energy Harvesting}

Scott K. Cushing

Follow this and additional works at: https://researchrepository.wvu.edu/etd

\section{Recommended Citation}

Cushing, Scott K., "Plasmonic Enhancement Mechanisms in Solar Energy Harvesting" (2015). Graduate Theses, Dissertations, and Problem Reports. 5423.

https://researchrepository.wvu.edu/etd/5423

This Dissertation is protected by copyright and/or related rights. It has been brought to you by the The Research Repository @ WVU with permission from the rights-holder(s). You are free to use this Dissertation in any way that is permitted by the copyright and related rights legislation that applies to your use. For other uses you must obtain permission from the rights-holder(s) directly, unless additional rights are indicated by a Creative Commons license in the record and/ or on the work itself. This Dissertation has been accepted for inclusion in WVU Graduate Theses, Dissertations, and Problem Reports collection by an authorized administrator of The Research Repository @ WVU.

For more information, please contact researchrepository@mail.wvu.edu. 


\title{
Plasmonic Enhancement Mechanisms in Solar Energy Harvesting
}

\author{
Scott K. Cushing
}

\author{
Dissertation submitted to the \\ Eberly College of Arts and Sciences \\ at West Virginia University \\ in partial fulfillment of requirements \\ for the degree of \\ Doctor of Philosophy \\ in \\ Physics
}

\author{
Alan Bristow, Ph. D., Chair \\ Nianqiang Wu, Ph. D., Co-Chair \\ Aldo Romero, Ph. D. \\ Cheng Cen, Ph. D. \\ Lawrence Hornak, Ph. D. \\ Department of Physics and Astronomy \\ Morgantown, West Virginia \\ 2015
}

Keywords: solar energy, plasmonics, photovoltaics, photocatalysis, water splitting, hydrogen generation, scattering, light trapping, hot electrons, hot carriers, PIRET, resonant energy transfer, local field enhancement, dephasing, nanoparticles 


\section{Abstract}

\section{Plasmonic Enhancement Mechanisms in Solar Energy Harvesting}

\section{Scott K. Cushing}

Semiconductor photovoltaics (solar-to-electrical) and photocatalysis (solar-to-chemical) requires sunlight to be converted into excited charge carriers with sufficient lifetimes and mobility to drive a current or photoreaction. Thin semiconductor films are necessary to reduce the charge recombination and mobility losses, but thin films also limit light absorption, reducing the solar energy conversion efficiency. Further, in photocatalysis, the band edges of semiconductor must straddle the redox potentials of a photochemical reaction, reducing light absorption to half the solar spectrum in water splitting. Plasmonics transforms metal nanoparticles into antennas with resonances tuneable across the solar spectrum. If energy can be transferred from the plasmon to the semiconductor, light absorption in the semiconductor can be increased in thin films and occur at energies smaller than the band gap.

This thesis investigates why, despite this potential, plasmonic solar energy harvesting techniques rarely appear in top performing solar architectures. To accomplish this goal, the possible plasmonic enhancement mechanisms for solar energy conversion were identified, isolated, and optimized by combining systematic sample design with transient absorption spectroscopy, photoelectrochemical and photocatalytic testing, and theoretical development. Specifically, metal@semiconductor nanostructures were designed to modulate the plasmon's scattering, hot carrier, and near field interactions as well as remove heating and self-catalysis effects. Transient absorption spectroscopy then revealed how the structure design affected energy and charge carrier transfer between metal and semiconductor. Correlating this data with wavelength-dependent photoconversion efficiencies and theoretical developments regarding metal-semiconductor interactions identified the origin of the plasmonic enhancement.

Using this methodology, it has first been proven that three plasmonic enhancement routes are possible: i) increasing light absorption in the semiconductor by light trapping through scattering, ii) transferring hot carriers from metal to semiconductor after light absorption in the metal, and iii) non-radiative excitation of interband transitions in the semiconductor by plasmon-induced resonant energy transfer (PIRET). The effects of the metal on charge transport and carrier recombination were also revealed. Next, it has been shown that the strength and balance of the three enhancement mechanisms is rooted in the plasmon's dephasing time, or how long it takes the collective electron oscillations to stop being collective. The importance of coherent effects in plasmonic enhancement is also shown. Based on these findings, a thermodynamic balance framework has been used to predict the theoretical maximum efficiency of solar energy conversion in plasmonic metal-semiconductor heterojunctions. These calculations have revealed how plasmonics is best used to address the different light absorption problems in semiconductors, and that not taking into account the plasmon's dephasing is the origin of low plasmonic enhancement Finally, to prove these guidelines, each of the three enhancement mechanisms has been translated into optimal device geometries, showing the plasmon's potential for solar energy harvesting.

This dissertation identifies the three possible plasmonic enhancement mechanisms for the first time, discovering a new enhancement mechanism (PIRET) in the process. It has also been shown for the first time that the various plasmon-semiconductor interactions could be rooted in the plasmon's dephasing. This has allowed for the first maximum efficiency estimates which have combined all three enhancement mechanisms to be performed, and revealed that changes in the plasmon's dephasing leads to the disparity in reported plasmonic enhancements. These findings are combined to create optimal device design guidelines, which are proven by fabrication of several devices with top efficiencies in plasmonic solar energy conversion. The knowledge obtained will guide the design of efficient photovoltaics and photocatalysts, helping usher in a renewable energy economy and address current needs of climate change. 


\section{Acknowledgements}

There are many and numerous thanks to be given upon the completion of this thesis. First and foremost, I must thank my advisors Dr. Nianqiang Wu and Dr. Alan Bristow. Without their constant guidance this thesis would have never reached fruition, let alone the current level it is at. Although not an advisor, I must also give my most sincere thanks to Dr. Jiangtian $\mathrm{Li}$, a postdoctor in Dr. Wu's group. Dr. Li joined our university right as Dr. Wu and I were planning the samples needed to separate the plasmonic enhancement mechanisms, and without his beautiful structures and magical ability to synthesize whatever my mind could think of, this thesis and work could never be completed. Dr. Li's abilities are on display throughout this thesis, and I thank him for the many years of fruitful collaboration. Along with Dr. Li, there are myriad people in both Wu's and Bristow's lab groups which I owe my gratitude, too many to list from my 4 years undergraduate and 4 years graduate at West Virginia University without leaving off names. Similarly to the Shared Research Facilities and University Staff. My thanks go out continually for your support, and if I do not get a chance to thank you in person before you read this thesis- again, thank you.

On a more personal note, I must also sincerely thank my parents for not only raising me into what I am today, but also ignoring the messes and broken experiments that still fill our garage, and understanding my absence from home while chasing my love for science. To my other friends and family, if there is any difference between the two, I am similarly grateful. You all have kept me laughing. Finally, I must extend my gratitude to my thesis-spanning girlfriend, Madeline Vandevender. When I first started as a scientist, I thought that the forwards in textbooks and papers thanking their husband or wife was simply a nice statement. Now I know that without these kind of people in your life, whether commiserating about a broken experiment, keeping me awake late in the lab, or pulling shifts to help edit a proposal the night before it is due- I could not be what I am. Thank you.

Thank you to God for watching over me, thank you all for the laughter and love, and thank you to any future reader that may come upon this thesis. I hope you enjoy the journey as much as I did. 


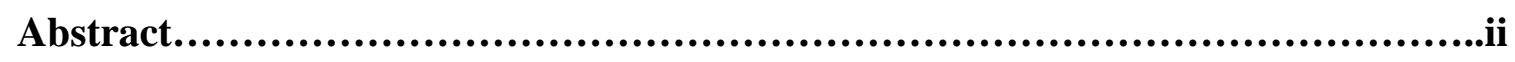

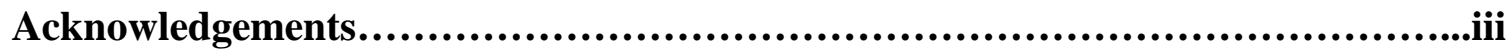

Table of Contents......................................................................

1. Introduction: Plasmonic Enhancement Mechanisms of Solar Energy Harvesting

1.1 Solar Energy: Semiconductor Photovoltaics and Photocatalysis..........................1

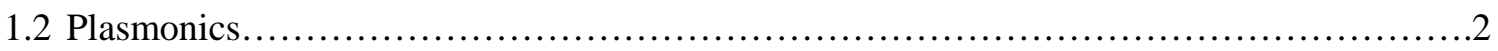

1.3 Confusion in How a Plasmon can Enhance Solar Energy Conversion........................ 7

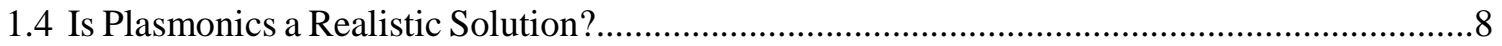

1.5 Summary and Goal of Thesis ......................................................

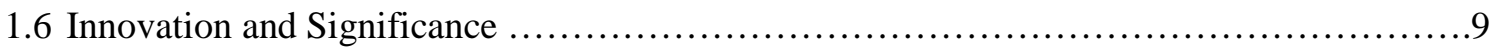

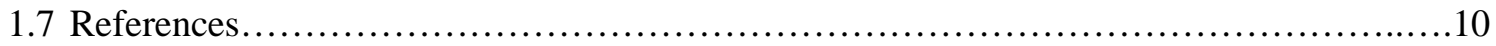

\section{Light Trapping Enhancement}

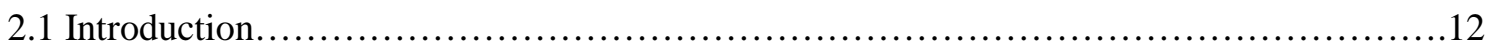

2.2 LSPR: Balancing Back-Scattering versus Plasmonic Absorption.........................13

2.3 SPP: High Surface Area, High Light Trapping Geometry................................15

2.3.1 Methods................................................................ 16

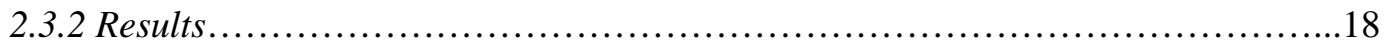

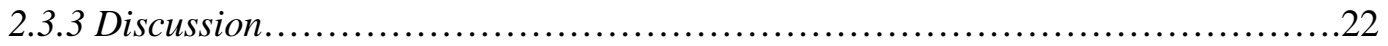

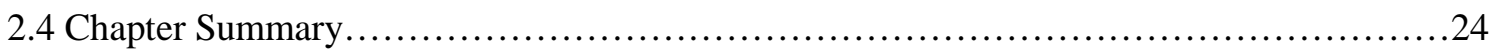

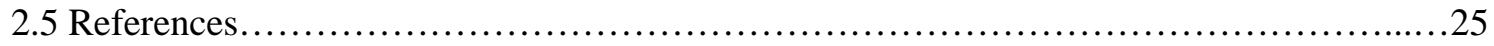

\section{Hot Carrier Enhancement}

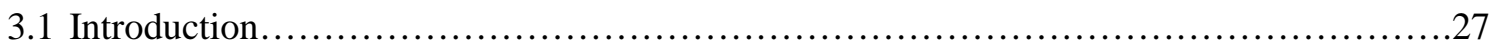

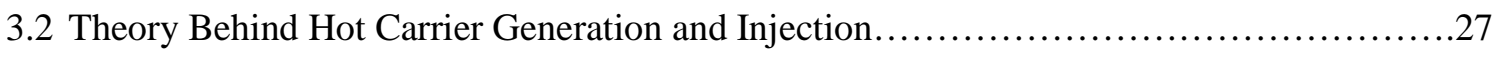

3.3 Gold Nanoparticles: Electron Relay versus Hot Electron Source........................29

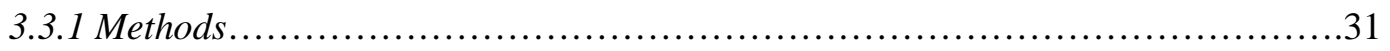

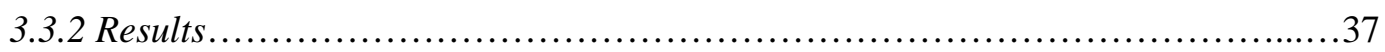

3.3.3 Discussion.................................................................

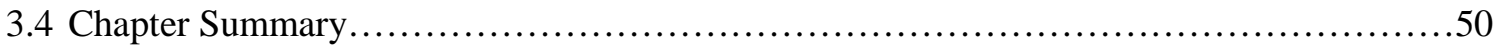

3.5 References..................................................................... 51

\section{Near Field Enhancement}

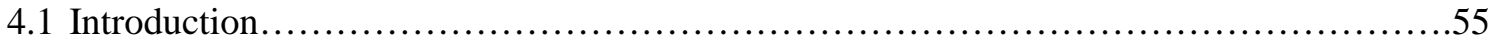

4.2 Isolating the Near-Field Plasmonic Enhancement Mechanism...............................55

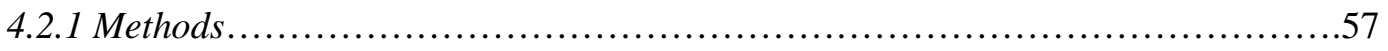

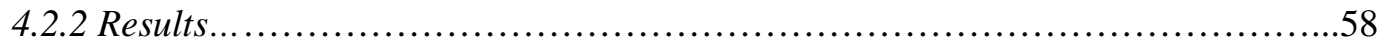

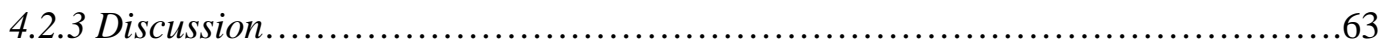

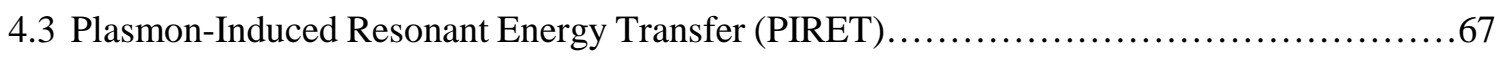

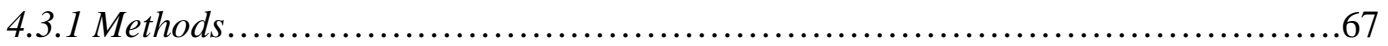




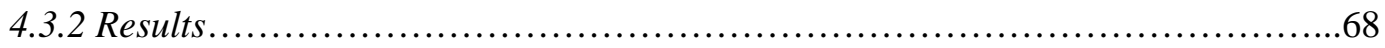

4.3.3 Discussion................................................................ 76

4.3.4 Supplementary Calculations............................................ 77

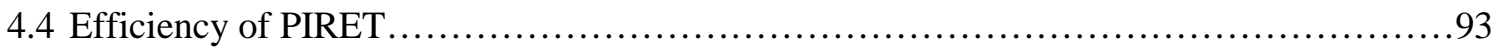

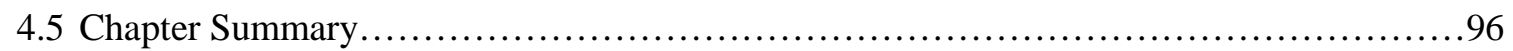

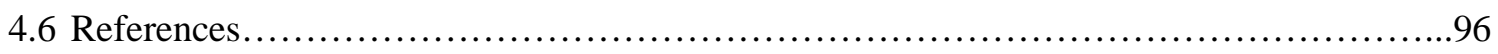

\section{Outlook: Dephasing, Control, and Maximum Efficiency}

5.1 Introduction................................................................99

5.2 Dephasing and Maximum Efficiency of Plasmonic Enhancement........................99

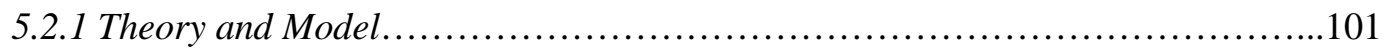

5.2 .2 Results................................................................106

5.2 .3 Discussion................................................................... 110

5.2.4 Supplementary Calculations............................................. 112

5.3 Engineering and Controlling Plasmonic Enhancement Mechanisms......................115

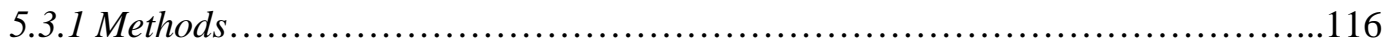

5.3 .2 Results...................................................................

5.3 .3 Discussion............................................................ 120

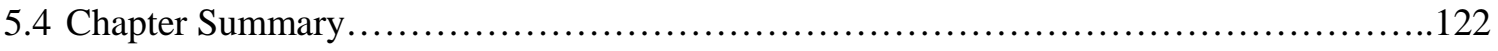

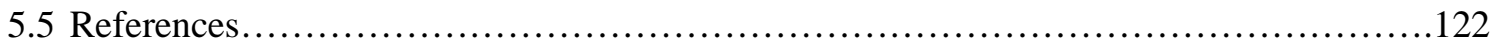

\section{Conclusions}

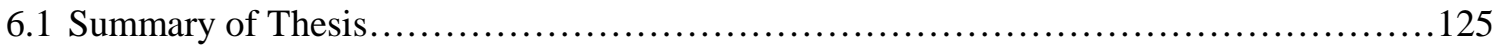

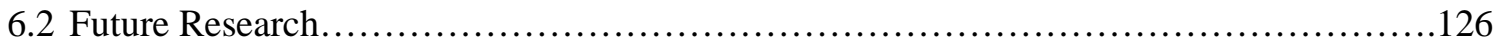

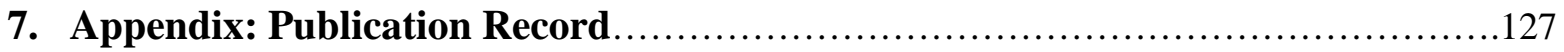




\section{Chapter 1: Introduction}

\subsection{Solar Energy: Semiconductor Photovoltaics and Photocatalysis}

A renewable, clean energy source must be found to meet the pressing issues of climate change and the world's ever increasing energy demands. Solar energy is one of the most promising solutions. ${ }^{1-6}$ The sun provides 173,000 TW of energy to Earth every day, of which only $13 \mathrm{TW}$ would be needed to meet current energy demands. ${ }^{7}$ The difficulty of solar energy is therefore not finding the energy source, but rather converting sunlight at a cost that is competitive with standard fuel sources to prompt global adoption.

Solar energy can be directly converted into electricity through photovoltaics, ${ }^{1-3}$ or converted into a fuel for storage and later use through photocatalysis. ${ }^{4-6}$ In both techniques a semiconductor absorbs incident light to promote an electron across the valence to conduction band energy gap, creating an excited electron hole pair (Figure 1.1a). In photovoltaics, the excited electron and hole pair must be spatially separated to drive a current, with the voltage given by the energetic offset of valence to conduction band. In photocatalysis, the excited electron hole pair must also be separated, but now to drive a redox reaction with the driving potential given by the offset of $\mathrm{VB}$ and $\mathrm{CB}$ from the redox potentials of the reaction.
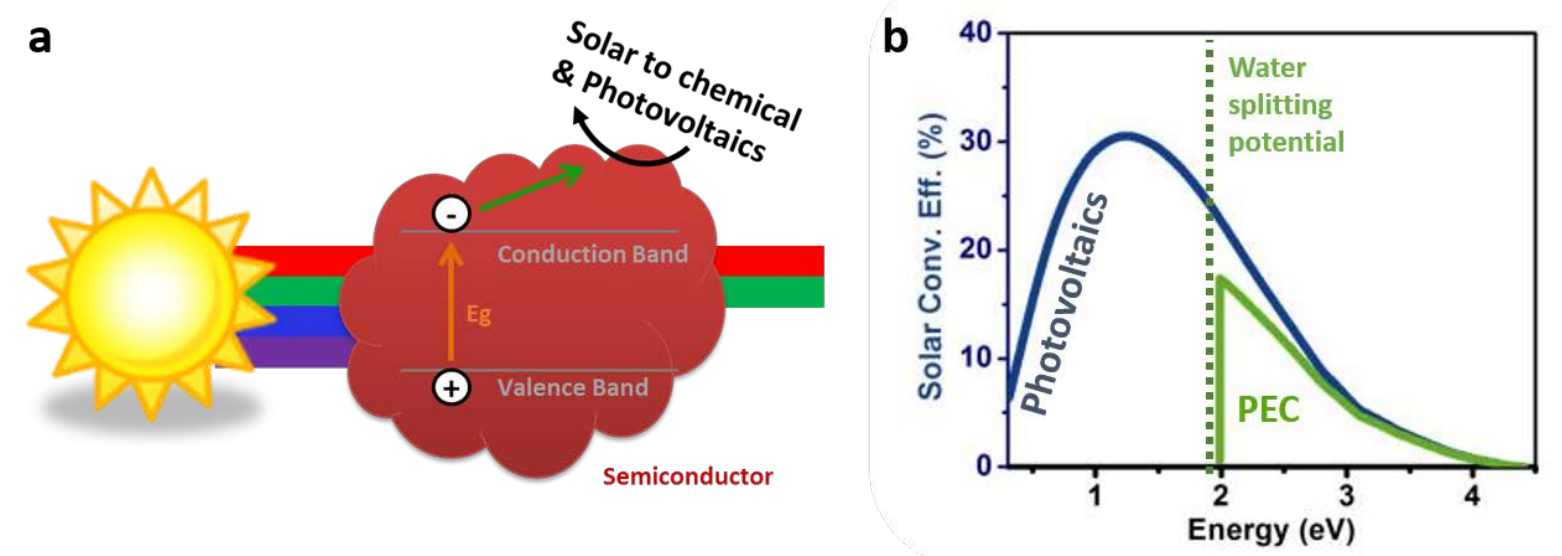

Figure 1.1. Solar energy conversion. a, Sunlight with energy larger than the semiconductor band gap can excite electron-hole pairs in the semiconductor, allowing for conversion of solar energy to electricity or a fuel. $\mathbf{b}$, The maximum thermodynamic efficiency of solar-to-electrical and solar-to-chemical conversion, balancing band gap energy against thermalization, calculated using the AM0 standard.

The efficiency of photovoltaic and photocatalytic devices have similar origin. Efficient solar energy harvesting hinges on i) a high absorption coefficient across the solar spectrum, ii) excitation of carriers with long lifetimes before recombination, and iii) excitation of carriers with high mobility. ${ }^{8}$ However, the maximum efficiency of the two solar energy conversion techniques differs because of how the excited carriers are utilized to create an output. In photovoltaics, the maximum efficiency is thermodynamically determined by the balance of energy lost by carriers with energy in excess of the semiconductor band gap versus light not absorbed at energies less than the band gap. ${ }^{9}$ Following the work of Shockley-Queisser, this leads to a maximum efficiency of $\sim 30 \%$ at $1.3 \mathrm{eV}$ for a single semiconductor (Figure 1.1b). In photocatalysis, the same thermalization issues exist, but the VB and CB must also match the redox potentials. ${ }^{10}$ This limits the band gap to $1.8-2 \mathrm{eV}$ for water splitting, which uses four photons to create hydrogen and oxygen fuels, and subsequently limits the maximum efficiency to $\sim 18 \%$. If the solar cell is expanded to be composed of two semiconductors, improving light absorption by allowing two band gaps 
and improving charge separation by the resulting p-n junction and built in potential gradient, the maximum efficiencies rise to $\sim 42 \%$ and $\sim 30 \%$ for photovoltaics and photocatalysis, respectively. ${ }^{11,12}$ If light concentration and three semiconductors are used, the efficiency for photovoltaics can rise to $\sim 60 \%{ }^{11}$

The idea of solar energy conversion through both photocatalysis and photovoltaics is not new. ${ }^{1-6}$ Years of research progress have actually allowed the thermodynamics limits in Figure $1.1 \mathrm{~b}$ to be approached. ${ }^{13}$ Yet, solar energy is still not widespread. This is because the increase in efficiency has come with a large subsequent increase in cost. The maximum efficiency limits in Figure $1.1 \mathrm{~b}$ are based only on the semiconductor's band gap, and assume 100\% light absorption and 100\% quantum yield on the conversion of absorbed photons to current or reaction. Obtaining near 100\% light absorption in practice requires thick semiconductor films. However, with thicker semiconductors come increased recombination and larger charge migration distances, balancing out any increase in efficiency.

Increased efficiency can thus only be achieved by either making thicker, higher crystallinity and defect free semiconductors, which is expensive, or using thinner, even nanostructured, semiconductor films coupled with charge extraction layers and small diffusion distances. ${ }^{8,14}$ The second approach, coupled with improved manufacturing techniques, has proven highly successful at raising efficiencies while continuing to drop module costs. ${ }^{15}$ However, another impasse has been met. As previous generation solar technologies mature and gains slow from addressing charge migration and recombination issues, the efficiency to cost ratio of solar energy has yet to meet the ratio needed to match traditional fossil fuel sources and achieve widespread commercial acceptance. ${ }^{14}$ This is because the thin semiconductor layers used to combat recombination issues and decrease manufacturing constraints on defects also decrease light absorption. Unlike recombination and migration issues, the light absorption per unit thickness is intrinsic to the semiconductor and cannot be easily changed. This issue is compounded in photocatalysis, for which the reaction's driving potential can already limit light absorption to half the solar spectrum.

The solution to this problem is to increase light absorption in current solar architectures without having to add complexity through doping new states or developing new materials which adds cost. One approach to solving this problem is to integrate an antenna into the solar cell. If the antenna had a smaller physical volume but larger absorption cross section than the same thickness of semiconductor, light could be captured, concentrated, and transferred to increase absorption while maintaining the thin films and nanoscale designs needed to combat charge migration and recombination. This goal can be met by plasmonics as discussed in the next section.

\subsection{Plasmonics}

The free conduction electrons of a metal are influenced by a time-dependent force opposite that of the changing electromagnetic field of the incident light (Figure 1.2a). The resulting motion of the electrons will be oscillatory, but 180 degrees out of phase due to the charge of the electron, and with damping caused by Ohmic losses. ${ }^{16}$ Like all oscillators, the conduction electrons have a characteristic frequency, in this case known as the plasma frequency ${ }^{16}$

$$
\omega_{p}=\sqrt{\frac{n e^{2}}{m_{e f f} \epsilon_{0}}} .
$$

The plasma frequency depends on the density of electrons $(n)$ and the effective mass $\left(m_{\text {eff }}\right)$, and corresponds to how easily the electrons can move in response to the incident field. Additionally, $e$ is the charge of an electron and $\epsilon_{0}$ is the permittivity of free space. ${ }^{16}$ On average, the free conduction 
electrons in the bulk of the metal do not oscillate against a restoring force, so unlike a mass on a spring, there is not a single resonant frequency. Instead the motion is similar to a mass being dragged in a viscous fluid, and will differ based on whether the electrons can respond quickly enough to the driving force of the incident field. If the light has a frequency above the plasma frequency (in the ultraviolet (UV) range for metals), the electrons will not oscillate and the light will simply be transmitted or absorbed in interband transitions. ${ }^{16}$ If the light has a frequency smaller than the UV range, the electrons will oscillate 180 degrees out of phase with the incident light, causing a strong reflection. ${ }^{17}$ The combination of plasma frequency and interband transitions gives metals their characteristic color. Mathematically this behavior is described by the real part of the dielectric constant $\left(\epsilon^{\prime}{ }_{\text {metal }}\right)^{17}$

$$
\frac{\epsilon_{\text {metal }}^{\prime}}{\epsilon_{0}}=1-\left(\frac{\omega_{p}}{\omega}\right)^{2}
$$

When the frequency of light is greater than the plasma frequency, the real part of the dielectric constant is positive and light is transmitted. ${ }^{17}$ When the frequency of light is less than the plasma frequency, the real part of the dielectric constant is negative, and the majority of light is reflected (Figure 1.2(d, e)). ${ }^{17}$ The dielectric constant therefore decides whether or not the metal electrons can oscillate at the given frequency of light. In Figure 1.2, the convention is used that the positive imaginary part of the dielectric constant corresponds to the Ohmic losses. To avoid confusion, it should be noted that the opposite convention of a negative imaginary part of the dielectric constant referring to loss is also found frequently in literature.

If the bulk metal is now shrunk to a thin film, the oscillations will only exist at the surface, leading to propagating charge waves known as surface plasmon polaritons (SPPs) (Figure 1.2b). ${ }^{18,19}$ The word "polariton" refers to the transformation of the bulk, volume oscillations to travelling surface charge waves. The interface between the metal $\left(\epsilon_{\text {metal }}\right)$ and the surrounding medium $\left(\epsilon_{\text {diel }}\right)$ places additional constraints on what frequencies the metal electrons can oscillate in the incident field. This limits the continuous spectrum of Equation 1.2 (all frequencies below $\omega_{p}$ ) for all incident angles to a fixed wave vector and frequency for a given interface. The resulting quantization is why the name is switched from plasma to plasmon.

The resonance condition to excite the SPP is given by ${ }^{18,19}$

$$
k_{S P P}=\frac{\omega}{\mathrm{c}} \cdot \sqrt{\frac{\epsilon_{\text {metal }} \cdot \epsilon_{\text {diel }}}{\epsilon_{\text {metal }}+\epsilon_{\text {diel }}}},
$$

which gives the dispersion curve for the SPP. The dispersion curve shows the wave vector of light necessary to excite a SPP for a given interface (Figure 1.2f). The wave vector or momentum of the oscillating charge wave is always greater than that of the massless photon. ${ }^{18,19}$ Therefore SPP cannot be directly excited by incident light, but can be only excited by a prism in the Kretschmann geometry, or by a grating to supply the extra momentum. ${ }^{1}$ The dispersion curve in Equation 1.3 gives the angle for which the grating or the prism can supply the necessary momentum to excite the SPP. At this angle, light will be absorbed, leading to a dip in the reflection or transmission spectrum (Figure 1.2g).

When the metal electrons oscillate, the real part of the dielectric constant is negative, therefore cancelling out the denominator in Equation 1.3, leading to a resonance condition at ${ }^{18,19}$ 


$$
\omega_{S P P}=\frac{\omega_{p}}{\sqrt{\left(1+\epsilon_{\text {diel }}\right)}}
$$

The dependence of the SPP frequency on the dielectric constant at the interface is what transforms the bulk plasma oscillations into a useful antenna. The local EM field resulting from the charge oscillations of the SPP extends $\sim 100-200 \mathrm{~nm}$ into the dielectric. ${ }^{20}$ If the local environment changes within this distance, the dielectric constant will differ, and the SPP frequency will shift from that in air. This can be understood conceptually as the dielectric screening the charge at the interface and reducing repulsion between adjacent electrons, effectively reducing the energy needed to drive oscillations and red-shifting the oscillation frequency. As the SPP frequency changes, so do both the dispersion curve and the angle at which the SPP can be excited, modulating the experimentally measured reflectance.
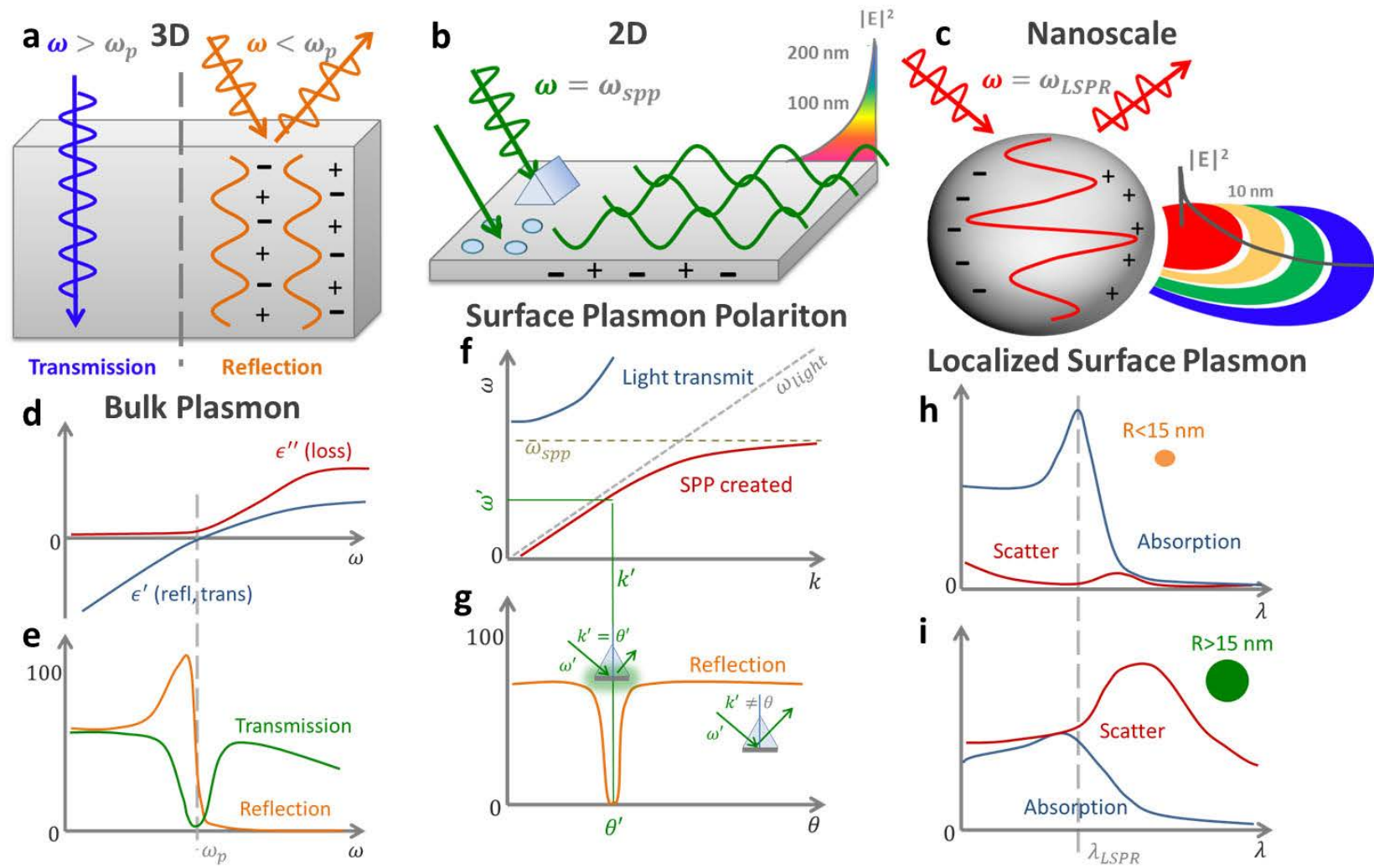

Figure 1.2. Volume, surface, and localized surface plasmon resonances. a, The plasma frequency of a metal describes the frequency below which the conduction electrons oscillate in the incident field. $\mathbf{d}$, These oscillations lead to a negative real part of the dielectric constant and, e, increased reflection from the metal. b, On a 2D surface, electron oscillations lead to propagating charge waves known as surface plasmon polaritons (SPPs). These oscillations are coupled to an electromagnetic field which propagates along the interface and with amplitude that exponentially decreases away from the interface. f, The SPP can only be excited at certain wave vectors and exists as a field that decays evanescently from the surface. g, The momentum matching condition leads to the SPP resonance only existing at certain incident angles. c, Localized surface plasmon resonance exists when the metal nanoparticle is smaller than the incident wavelength, making the electron oscillations in phase. The collective oscillations lead to a large absorption and scattering cross section, as well as an amplified local EM field. h, For small particles less than $\sim 15 \mathrm{~nm}$, the absorption dominates and the absorption cross-section is large. i, For nanoparticles greater than $\sim 15 \mathrm{~nm}$, the scattering cross-section dominates. The EM field is taken as polarized in the plane of incidence in the figures.

The narrow absorption line shape and high angular specificity of the SPP have allowed excellent signal-to-noise ratio and figure of merit to be obtained for SPP-based sensors, however the narrow 
linewidth limits the usability as an antenna for the broad solar spectrum. ${ }^{18}$ The SPP can achieve light confinements greater than the $4 n^{2}$ of dielectrics, but much of the incident energy is stored in the kinetic energy of the travelling polariton wave instead of the potential energy of the local field. ${ }^{21}$ This means most absorbed light is eventually turned into heat instead of allowing possible conversion into light absorption in the semiconductor, making an inefficient antenna. Further, the necessary coupling geometry to excite the SPP, whether a grating or external prism, greatly complicates solar cell construction, increasing costs and offsetting any benefit.

The restrictions of SPP can be overcome by changing a two-dimensional (2D) metal film to a nanoparticle. The incident electric field will be constant across the nanoparticle if it is smaller than the wavelength of light, inducing a uniform displacement of the electron density and a strong restoring force from the positive ionic core background (Figure 1.2c). ${ }^{22}$ The restoring force leads to a characteristic oscillation frequency in the metal electrons similar to a simple harmonic oscillator. This phenomenon is known as localized surface plasmon resonance (LSPR). ${ }^{22}$ LSPR can be excited directly by the incident field because the geometry of the nanoparticle supplies the additional momentum. ${ }^{22}$ The local environment-induced change in the LSPR peak position can therefore be detected using a simple UV-Visible spectrometer without need of additional gratings or prisms (Figure 1.2h).

The exact conditions for LSPR can be solved for a nanosphere using Mie theory or a simple harmonic oscillator model, ${ }^{23-25}$ as the extinction (absorption + scattering) cross-section is expressed,

$$
\sigma_{\text {ext }}=9\left(\frac{\omega}{c}\right)\left(\epsilon_{\text {diel }}\right)^{\frac{3}{2}} V \frac{\epsilon_{\text {metal }}^{\prime \prime}}{\left(\epsilon_{\text {metal }}^{\prime}+2 \epsilon_{\text {diel }}\right)^{2}+\left(\epsilon_{\text {metal }}^{\prime \prime}\right)^{2}},
$$

Equation 1.5 shows that when the electrons in the metal oscillate and the real part of the dielectric function is negative, the denominator will vanish, leading to a strong resonance condition at

$$
\omega_{L S P R}=\frac{\omega_{p}}{\sqrt{\left(1+2 \epsilon_{\text {diel }}\right)}},
$$

which will shift with change in the local dielectric environment. The coherent oscillations of the electrons make the absorption and scattering cross-section at resonance several orders of magnitude larger than the physical size of the nanoparticle, given by $V=4 / 3 \pi R^{3}$.

Several key differences exist between LSPR and SPP that must be taken into account when designing an antenna for solar energy. First, the factor of 2 in front of the interfacial dielectric constant depends on the geometry of the nanoparticle. The LSPR peak position will change with shape in addition to the metal used and the local environment (Figure 1.3). ${ }^{26-28}$ The larger the nanoparticles, the smaller the repulsion for electrons at opposite surfaces, and the more red-shifted the plasmon will be. ${ }^{29-31}$ Second, the confined electron oscillations in LSPR lead to an intense local EM field, which can be several orders of magnitude stronger than the incident field strength. In nanoparticles with sharp edges, the field will be concentrated similar to a lightning rod, increasing the local field intensity further and improving the possible coupling to a semiconductor (Figure 1.4). ${ }^{26-27,32,33}$ The EM field in LSPR decays in $~ 10-30 \mathrm{~nm}$ and is therefore more sensitive to changes in distance from the surface of the metal and the local refractive index of the surrounding environment than the SPP that decays in $\sim 100-200 \mathrm{~nm}$, and can also allow for higher localization of incident light, increasing the efficiency of the antenna on a per-volume basis. 

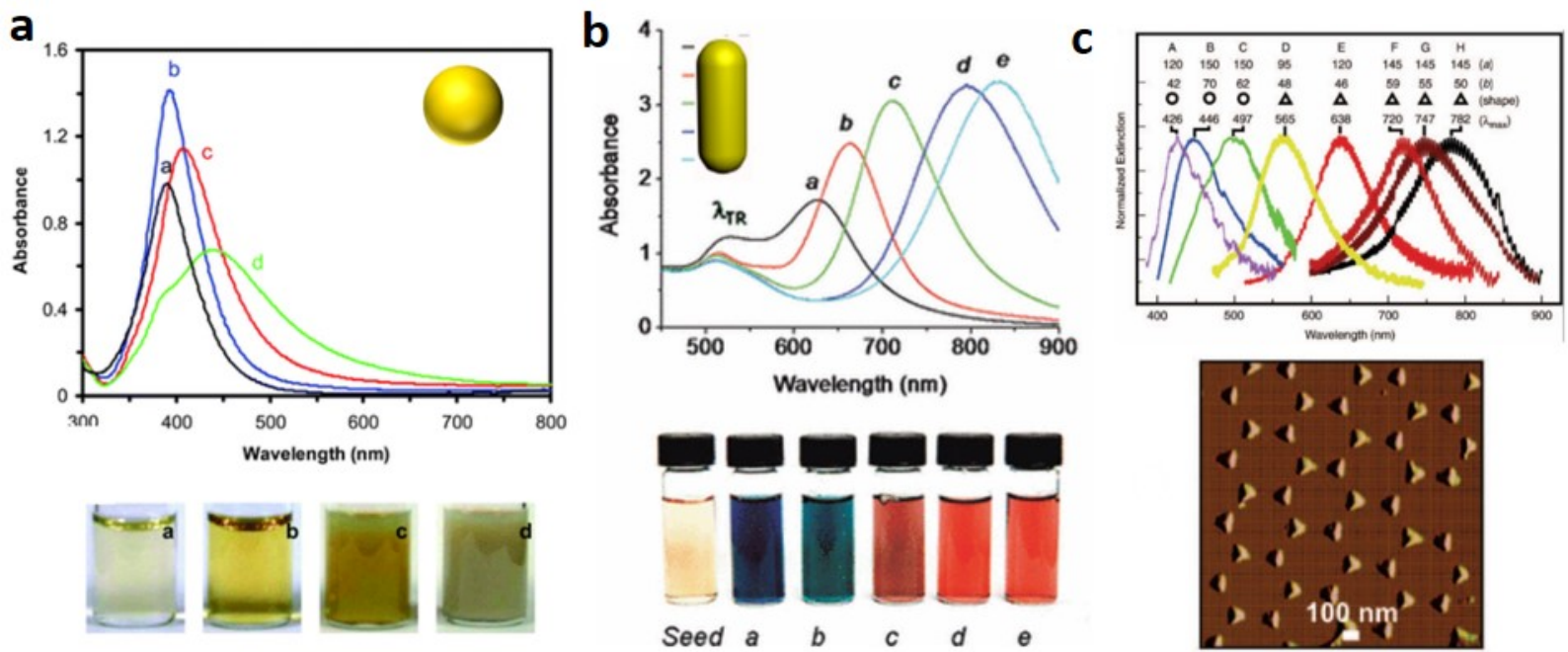

Figure 1.3. Representative plasmonic nanostructures for plasmonics. a, Extinction spectra (top), and optical images (bottom) of different sized Ag nanospheres in aqueous solutions (Reprinted with permission from ref. 29, Copyright 2005, the Royal Society of Chemistry), b, Au nanorods with various aspect ratios (Reprinted with permission from ref. 30, Copyright 2010, Elsevier B.V.), c, and various Ag triangle arrays prepared by nanosphere lithography Reprinted with permission from ref. 31, Copyright 2005, Materials Research Society.
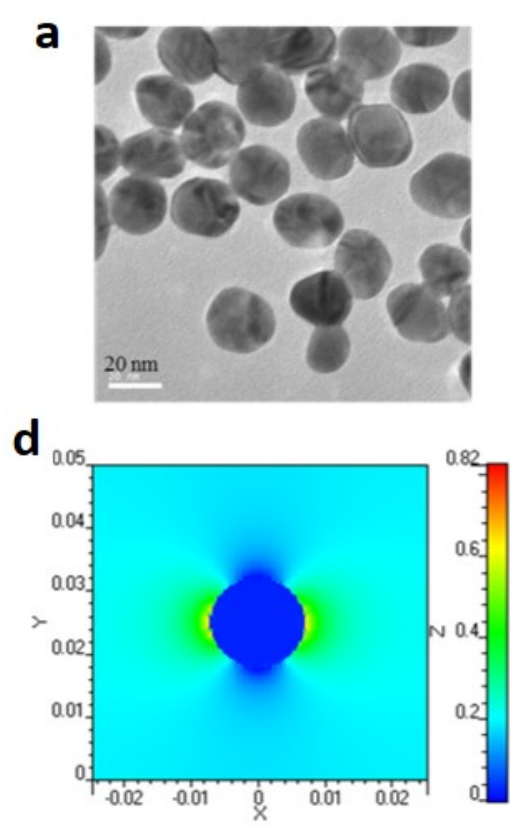

b

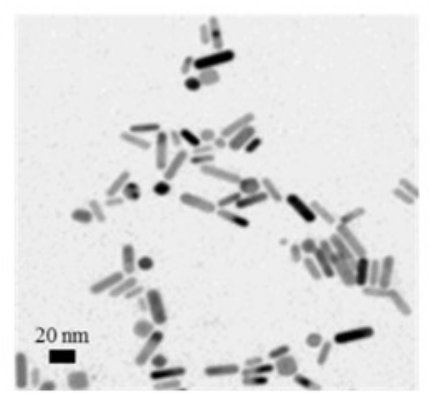

$\mathbf{e}_{0.05}$

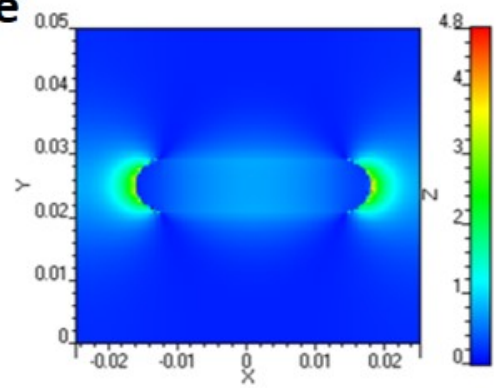

C

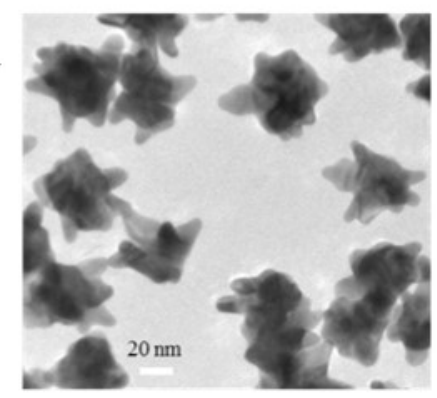

f

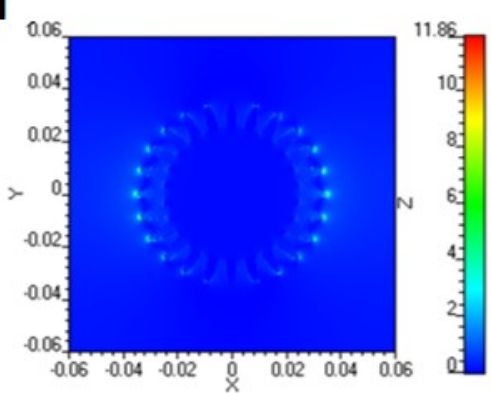

Figure 1.4. TEM images and electric field distributions of (a,d) Au nanosphere, (b,e) Au nanorod and (c,f) Au nanostar synthesized by wet-chemistry methods. Reprinted with permission from ref. 26, Copyright 2012, IOP Publishing.

Since the LSPR can be excited by incident light, the plasmon can also re-radiate its energy into the far field as scattering, with the size of the particle determining if absorption or scattering dominates (Figure 1.2(h, i)). ${ }^{23}$ In small metal nanoparticles (less than $\sim 15 \mathrm{~nm}$ ) electron-electron scattering quickly converts the energy of the LSPR into heat, which translates into a strong absorption. ${ }^{22,34,35}$ In larger particles the electron-electron surface scattering is reduced, and the 
energy of the plasmons will be re-radiated, leading to a strong scattering cross-section. ${ }^{22,34,35}$ The radiative damping and electron-electron scattering make the lifetime of the LSPR much shorter than that of the SPP. ${ }^{36}$ Since the spectral width is inversely related to the lifetime, LSPR has a broader absorption peak than SPP, making it ideal for capturing large amounts of the solar spectrum in a single antenna. The absorption line width can be narrowed or broadened by optimizing the geometry and using planar arrays of metal nanoparticles.

Finally, since both SPP and LSPR have local fields, coupling can occur between SPP/SPP, SPP/LSPR, and LSPR/LSPR when the supporting metal structures are brought within the local field decay length. ${ }^{18}$ The coupling can lead to an enhanced local field and shifting in the spectral position due to hybridization between the modes. ${ }^{37}$ For example, the local field enhancement of two spheres goes from $\sim 10$ to $\sim 10^{4}$ when they are aggregated. ${ }^{18,22}$ Therefore one of the largest design parameters in plasmonics is the geometry of the metal nanoantenna and its proximity to other metal nanoparticles. By tuning these components, the plasmon's optical response can be changed from absorber to scatterer, heat generation versus trapping energy in the local field, and this can be done over the full solar spectrum with tuneable cross sections.

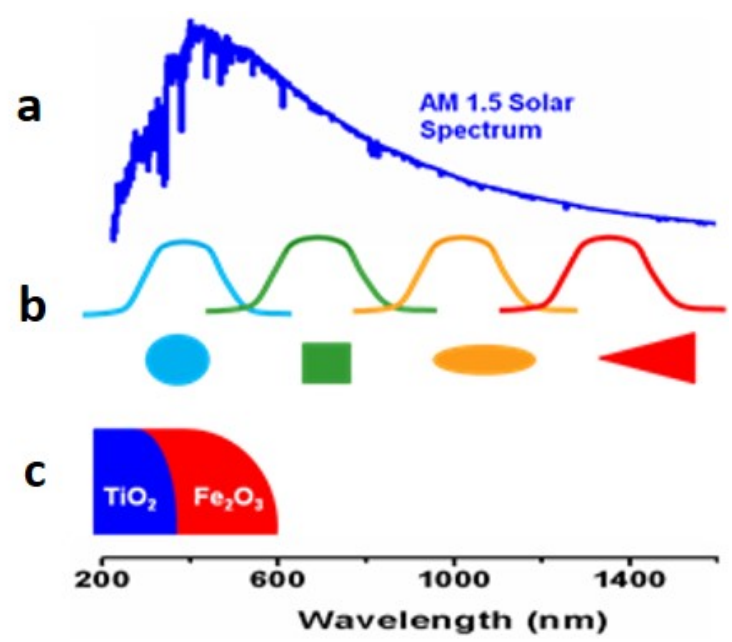

Figure 1.5. a, AM1.5G solar spectrum ${ }^{52}$, b, representative tuning range for LSPR showing the full solar spectrum harvesting with LSPR as a sensitizer, $\mathbf{c}$, and the absorbance of common semiconductors used in water splitting.

\subsection{Confusion in how a Plasmon can Enhance Solar Energy Conversion}

The tuneable optical properties of plasmonics and large localization volumes therefore make plasmonics ideal for enhancing the absorption issues which currently limit solar energy conversion (Figure 1.5). ${ }^{38-42}$ This potential was first seen in $\mathrm{TiO}_{2}$, wherein an increase in photocatalysis was found to follow the plasmon's absorption peak. ${ }^{43}$ Several different metal semiconductor combination confirmed this trend to be true, however confusion also arose as to how the plasmon could enhance light absorption in the semiconductor. ${ }^{44-46}$ When light is incident on the metal nanoparticle, responses of scattering, absorption in the metal, and the local field all co-exist with strength depending on the metal nanoparticles geometry, constituent metal, local environment, and resonance frequency. The plasmon therefore has multiple enhancement and interaction pathways with the semiconductor, all of which can co-exist, and all of which can occur on sub-picosecond time scales or even during the sub-10 femtosecond lifetime of the plasmon. ${ }^{47-51}$ 
This complexity was multiplied by the confluence of various plasmonic architectures in both photovoltaics and photocatalysis being studied, and the fact that the enhancement mechanism was commonly extrapolated between initial plasmon absorption and final solar energy enhancement. This was done despite the influence of semiconductor defects, self-catalysis and charge trapping effects of the metal, carrier lifetimes, and mobility that affect excited carrier distributions between these two steps. Beyond debate about how the plasmon was enhancing solar energy conversion, an even larger issue was raised:

\subsection{Is Plasmonics a Realistic Solution?}

The first dissent of any non-proponent of plasmonics is that the research is useless for application because of the cost of the precious metals used. While large area, self-assembled, and highthroughput fabrication has made mass-producing metal nanoparticles and patterns an obtainable goal at low costs, the issue of raw materials remains. Given that current solar energy research is not so much concerned with efficiency as cost, how can adding precious metals like silver and gold help?

This question is best answered with a rudimentary cost-analysis to offset the ingrained assumption that jewellery cannot make a good solar cell. The following chapters will show that once, and only once, the plasmonic enhancement mechanisms are properly engineered, the efficiency of a moderately absorbing solar cell can be increased two to three times. The average plasmonic nanoparticle is $\sim 10 \mathrm{~nm}$ in diameter, or assuming a square shape, has a volume of $10^{-21}$ L. A gold band or ring of diameter $\sim 4 \mathrm{~mm}$ has a volume of $\sim 5 \mu \mathrm{L}$. Assuming a plasmonic nanoparticle coverage of $25 \%$, a common balance to prevent surface coverage and charge blocking issues, one gold ring could cover $\sim 2 \mathrm{~m}^{2}$ of solar cell with plasmonic nanoparticles.

The cost of an average $1 \mathrm{~m}^{2}$ solar panel is $\sim \$ 500$ dollars installed gives an output of $150-200$ Watts. ${ }^{7}$ This leads to the familiar figure of merit of $3 \$ \mathrm{~W}$ used when judging the cost effectiveness of solar energy. If the gold ring, which we will assume costs a reasonable $\$ 150$ at the store to cover manufacturing and design costs as well as raw materials, is turned into nanoparticles the efficiency of the solar panel double to triples. This puts the output for $1 \mathrm{~m}^{2}$ at $400-600 \mathrm{~W}$ per hour for a cost of half the ring, or $\$ 75$ since $2 \mathrm{~m}^{2}$ can be coated. The new figure of merit is now 1-2 $\$ / \mathrm{W}$ per hour. Converting this to a comparison with gasoline is more complicated given the up-front cost of solar versus long-term cost of gasoline, but the message is clear even from this simple analysis: Adding precious metal nanoparticles has the potential to decrease the cost per output of solar cells.

\subsection{Summary and Goal of Thesis}

When this thesis was first formulated, the goal was to systematically identify and isolate the different possible plasmonic enhancement mechanisms for solar energy conversion. In the following chapters, not only is that achieved, but the importance of dephasing and coherent effects in linking the three discovered enhancement mechanisms and their transfer efficiencies is revealed. Based on these results, the maximum efficiency of plasmonic solar energy harvesting is predicted within a Shockley-Queisser framework, providing design guidelines which are then proven in several fabricated, high efficiency devices.

The central motive between each chapter is the combination of systematic sample design engineering nanostructures which isolate and eliminate different plasmonic responses and 
background processes - with instrumentation development and performance testing designed to monitor the plasmonic enhancement from initial light absorption to final excited carrier extraction. The ideal tool for this goal proved to be transient absorption spectroscopy, filling the gap of femtosecond to nanosecond evolution that was missing from other plasmonic studies of the time. Transient absorption spectroscopy was adapted to uniquely determine the plasmonic enhancement mechanism, measure effects of coherences, and determine how and where carriers were being deposited in the semiconductor. The transient absorption results were also heavily influenced by a suite of other tools ranging from simple UV-Visible spectroscopy to photoelectrochemical testing, as well as theoretical prediction and development ranging from finite difference time domain simulations to quantum master equation calculations using the density matrix.

Beyond this central motive, the thesis is structured into two parts. First, the results are organized into three main chapters which cover the three plasmonic enhancement mechanisms discovered. In each chapter a full outlook on how, why, and what the expected efficiency of that aspect of the plasmon is given along with guidelines for implementation, as proven in fabricated and tested devices. Second, the thesis is ended by a chapter focusing on the most recent advances I have made: including the effects of the plasmon's dephasing and coherences, calculations of the maximum plasmonic enhancement, and guidelines for designing nanostructures for high performance plasmonic solar energy conversion. Whereas the findings of the first part of the thesis acts almost as a review for how plasmonic solar energy transfer can occur, the findings of the second part of the thesis act as an outlook for future work and proof of the still far reaching applicability of plasmonics.

\subsection{Innovation and Significance}

This dissertation has made several advances in the field of plasmonics with direct impact on the field of solar energy conversion. First, the three possible plasmonic enhancement mechanisms were identified and isolated for the first time. In doing so, a new enhancement mechanism (PIRET) was discovered, explored, and understood both theoretically and experimentally. PIRET was shown to have higher near and below band edge photoconversion enhancements than other plasmonic effects, extending solar energy harvesting ranges by several hundred nanometers with efficiency equal to UV excitation. How to optimize and tune all three enhancement mechanisms was shown and proven by implementation in several high performance plasmonic photocatalysis devices. This included clarifying and utilizing the effects of metal nanoparticles on charge transport and excited carrier lifetimes.

It was also shown for the first time that the various plasmon-semiconductor interactions could be rooted in the plasmon's dephasing and that coherent effects are critical for plasmonic enhancement mechanisms. This allowed for the first maximum efficiency estimates which combined all three enhancement mechanisms to be performed, and revealed that changes in the plasmon's dephasing leads to the disparity in reported plasmonic enhancements. These calculations also showed that, by optimizing the plasmon's dephasing, plasmonics has the potential to improve semiconductor photoconversion by two to three times across the solar spectrum. These calculations were used to create optimal device design guidelines, which were proven by fabrication of several devices with top efficiencies in plasmonic solar energy conversion. The results of this dissertation will allow for more efficient photovoltaics and photocatalysts to be created, helping usher in a renewable energy economy and address current environmental needs. 


\subsection{References}

1. Green, M. A. Solar cells: operating principles, technology, and system applications; Prentics Hall: New Jersey, 1982.

2. Grätzel, M. Inorg. Chem. 2005, 44, 6841-6851.

3. Shah, A.; Torres, P.; Tscharner, R.; Wyrsch, N.; Keppner. H. Science 1999, 285, 692-698.

4. Walter, M. G.; Warren, E. L.; McKone, J. R.; Boettcher, S. W.; Mi, Q.; Santori, E. A.; Lewis, N. S. Chem. Rev. 2010, 110, 6446-6473.

5. Ni, M.; Leung, M. K.; Leung, D. Y.; Sumathy, K. Renew. Sust. Energ. Rev. 2007, 11, 401-425.

6. Bolton, J. R. Solar Energy, 1996, 57, 37-50.

7. Lewis, N. S. MRS Bulletin 2007, 32, 808-820.

8. Li, J.; Wu, N. Catal. Sci. Tech. 2015, 5, 1360-1384.

9. Shockley, W.; Queisser, H. J. J. Appl. Phys. 1961, 32, 510-519.

10. Bolton, J. R.; Strickler, S. J.; Connolly, J. S. Nature 1985, 316, 495.

11. De Vos, A. J. Phys. D: Appl. Phys. 1980, 13, 839.

12. Hu, S.; Xiang, C.; Haussener, S.; Berger, A. D.; Lewis, N. S. Energy Env. Sci. 2013, 6, 2984-2993.

13. http://www.nrel.gov/ncpv/images/efficiency_chart.jpg (accessed October, 2015)

14. Beard, M. C.; Luther, J. M.; Nozik, A. J. Nature Nanotech. 2014, 9, 951-954.

15. http://www.forbes.com/sites/peterdiamandis/2014/09/02/solar-energy-revolution-a-massiveopportunity/ (accessed October, 2015)

16. Ashcroft, N. W.; Mermin, D. N. Solid Stat. Physics; Cengage Learning: Boston, 1976.

17. Oates, T. W. H.; Wormeester, H.; Arwin, H. Prog. Surf. Sci. 2011, 86, 328-376.

18. Lackowicz, J. R., Plasmonics 2006, 1, 5-33.

19. Dostalek, J.; Knoll, W. Biointerphases 2008, 3, 12-22.

20. Shalaev, V. M.; Kawata, S. Nanophotonics with Surface Plasmon; Elsevier: Atlanta, 2007.

21. Munday, J. N.; Callahan, D. M.; Atwater, H. A. Appl. Phys. Lett. 2012, 100, 121121.

22. Mayer, K. M.; Hafner, J. H. Chem. Rev. 2011, 111, 3828-3857.

23. Li, Y.; Zhao, K.; Sobhani, H.; Bao, K.; Nordlander, P. J. Phys. Chem. Lett. 2013, 4, 13521357.

24. Mulvaney, P. Langmuir 1996, 12, 788-800.

25. Schwartzberg, A.M.; Zhang, J. Z.; J. Phys. Chem. C. 2008, 112, 10323-10337.

26. Li, M.; Cushing, S. K.; Zhang, J.; Lankford, J.; Aguilar, Z. P.; Ma, D.; Wu, N. Nanotechnology 2012, 23, 115501.

27. Willets, K. A.; Van Duyne, R. P. Annu. Rev. Phys. Chem. 2007, 58, 267-297.

28. Lee, K. S.; El-Sayed, M. A. J. Phys.Chem. B 2005, 109, 20331-20338.

29. Nallathamby, P. D.; Huang, T.; Xu, X. H. N. Nanoscale 2010, 2, 1715-1722.

30. Tong, L.; Wei, Q.; Wei, A.; Cheng, J. X. Photochem. Photobiol. 2009, 85, 21-32.

31. Haes, A. J.; Haynes, C. L.; McFarland, A. D.; Schatz, G. C.; Van Duyne, R. P.; Zou, S. MRS Bull. 2005, 30, 368-375.

32. Li, M.; Gou, H.; Al-Ogaidi, I.; Wu, N. ACS Sustainable Chem. Eng. 2013, 1, 713-723.

33. Wang, H.; Levin, C. S.; Halas, N. J. J. Am. Chem. Soc. 2005, 127, 14992-14993.

34. Gryczynski, I.; Malicka, J.; Gryczynski, Z.; Lakowicz, J. R. Anal. Biochem. 2004, 324, 170182.

35. Lakowicz, J. R. Anal. Biochem. 2005, 337, 171-194. 
36. Sönnichsen, C.; Franzl, T.; Wilk, T.; Von Plessen, G.; Feldmann, J. Phys. Rev. Lett. 2002, 88, 077402.

37. Prodan, E.; Radloff, C.; Halas, N. J.; Nordlander, P. Science 2003, 17, 419-422.

38. Atwater, H. A.; Polman, A. Nat. Mater. 2010, 9, 205-213.

39. Linic, S.; Christopher, P.; Ingram, D. B. Nat. Mater. 2011, 10, 911-921.

40. Brongersma, M. L.; Halas, N. J.; Nordlander, P. Nat. Nanotechnol. 2015, 10, 25-34.

41. Cushing, S. K.; Wu, N. Q. Interface 2013, 22, 63-67.

42. Warren, S. C.; Thimsen, E. Energy Env. Sci 2012, 5, 5133-5146.

43. Tian, Y.; Tatsuma, T. Chem. Commun. 2004, 16, 1810-1811.

44. Furube, A.; Du, L.; Hara, K.; Katoh, R.; Tachiya, M. J. Am. Chem. Soc. 2007, 129, 14852-14853.

45. Awazu, K.; Fujimaki, M.; Rockstuhl, C.; Tominaga, J.; Murakami, H.; Ohki, Y.; Yoshida, N.; Watanabe, T. A J. Am. Chem. Soc. 2008, 130, 1676-1680.

46. Standridge, S. D.; Schatz, G. C.; Hupp, J. T. J. Am. Chem. Soc. 2009, 131, 8407-8409.

47. Kelly, K. L.; Coronado, E.; Zhao, L. L.; Schatz, G. C. J. Phys. Chem. B 2003, 107, 668-677.

48. Link, S.; El-Sayed, M. A. Int. Rev. Phys. Chem. 2000, 19, 409-453.

49. Sönnichsen, C.; Franzl, T.; Wilk, T.; von Plessen, G.; Feldmann, J.; Wilson, O.; Mulvaney, P. Phys. Rev. Lett. 2002, 88, 077402.

50. Link, S.; El-Sayed, M. A. J. Phys. Chem. B 1999, 103, 4212-4217.

51. Link, S.; El-Sayed, M. A. J. Phys. Chem. B 1999, 103, 8410-8426.

52. http://rredc.nrel.gov/solar/spectra/am1.5/ (accessed October, 2015) 


\section{Chapter 2: Light Trapping Enhancement}

\subsection{Introduction}

As mentioned in Chapter 1, the plasmon's scattering can be used to increase absorption in the semiconductor at energies above the semiconductor band gap. ${ }^{1-8}$ Thin semiconductor films or nanostructures are required to balance recombination losses as well as increase surface area in photovoltaic, photoelectrochemical, and photocatalytic devices. By using the plasmon's large scattering cross section, the path length of the photon can be increased through multiple reflections, increasing the probability it is absorbed. This allows for device geometries with decreased thickness and recombination losses but increased absorption, increasing solar energy conversion.

Plasmonic light trapping through tuneable broad-band reflectors, as popularized by Atwater and Polman, ${ }^{9}$ has proven highly successful in increasing photoconversion efficiencies. Plasmonic light trapping usually occurs through two geometries, shown in Figure 2.1. In the first case, plasmonic nanoparticles with high scattering coefficients are placed within the bulk of the semiconductor, with multiple subsequent scattering events increasing the light absorption probability in the semiconductor. In the second case, a patterned or textured plasmonic back-reflector is used to reflect light through the cell like a mirror, or trap it at the metal-semiconductor interface. Several combinations of these two cases exist, allowing "black" plasmonic patterns which can trap $~ 90 \%$ of incident light across the solar spectrum. ${ }^{9}$ Additionally, by controlling where the light is localized within the semiconductor the excitation of carriers can be spatially controlled, further increasing efficiencies.

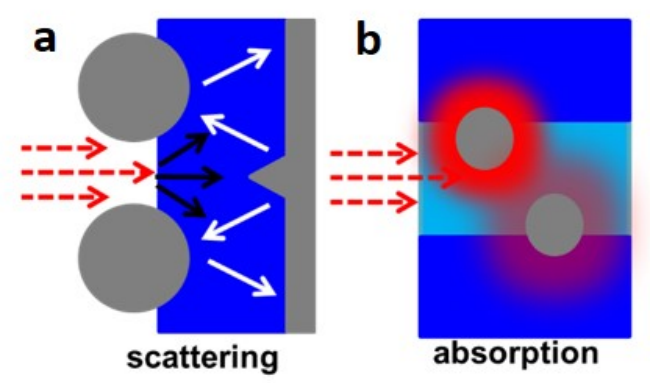

Figure 2.1. Light trapping enhancement in plasmonic metal-semiconductor hetero-structures, including a, increasing the optical path length through scattering and $\mathbf{b}$, increasing the absorption cross section by localizing the incident field.

All variants of light trapping schemes have the same goal: increasing absorption of the semiconductor at energies above the band gap. However, the existence of a plasmon in a metal nanoparticle at the proper resonance frequency does not guarantee an increase in solar energy conversion efficiency. First, the size of the plasmonic nanoparticle or textured back-reflector elements determines if light is absorbed in the metal, focused in the near field, or scattered (see Chapter 1 and Figure 2.2). This balance is not trivial to determine by UV-Vis reflection measurements alone, and care must be taken that the measured light trapping is not just the metal absorbing incident light, converting it to heat and wasting energy. The spatial position of the metal nanoparticles must also be such that reflected light can be completely absorbed and the metal nanoparticles do not shadow the remaining semiconductor. Second, the metal nanoparticle or back-reflector must be integrated into the PEC or PV architecture without impeding flow of carriers or acting as charge 
traps. This is particularly difficult to achieve in PEC and photocatalysts, as large surface is necessary for high efficiency solar-to-chemical conversion. These two problems are addressed in the following sections.

\subsection{LSPR: Balancing Back-Scattering versus Plasmonic Absorption}

The absorption in the semiconductor can be enhanced at energies above the band gap, where the semiconductor can already absorb light on its own, through light-trapping by scattering and concentration of incident light in the plasmon's local field (Further discussed in Chapter 4). In both cases, the metal nanoparticles act as antennas, localizing light from a larger volume than their physical cross section. LSPR is primarily used in low-cost fabricated solar cells because metal nanoparticle integration is easy to implement during spin-coating or deposition without impacting the semiconductor's crystalline structure.

For this approach to be successful the plasmon's absorption versus scattering and the position of the metal nanoparticle within the solar cell must be optimized. The balance of scattering versus absorption by the plasmon depends explicitly on the metal nanoparticles volume because the radiation damping of the plasmon scales as the volume. ${ }^{10-12}$

These effects are further shown in Figure 2.2 using Mie theory as implemented in Mielab, ${ }^{13}$ which includes all multi-pole contributions to the plasmon resonance. A $15 \mathrm{~nm}$ sized gold nanoparticle, Figure 2.2a, primarily localizes the EM field as depicted in Figure 2.2c. A $100 \mathrm{~nm}$ sized particle primarily scatters the incident field, Figure 2.2b, in the forward and backward directions, Figure 2.2d. By tuning the size, shape, and geometry of the nanoparticle the absorption versus scattering can thus be completely controlled. Given that coupling through the near field (See Chapter 4) and light-trapping can approach $>80 \%$ efficiencies, the optimal approach depends more on where the plasmonic nanoparticles can be placed within the semiconductor without hurting charge recmbination than how the light is re-absorbed.

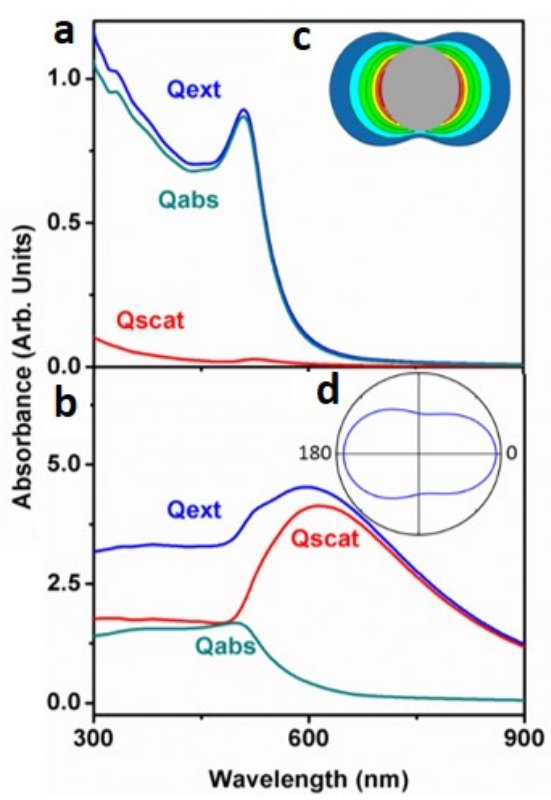

Figure 2.2 Extinction, scattering, and absorption for a, a $15 \mathrm{~nm}$ sized gold sphere and $\mathbf{b}$, a $100 \mathrm{~nm}$ sized gold sphere. As the sphere size increases the LSPR shifts from c, localizing to $\mathbf{d}$, scattering the incident EM field. 

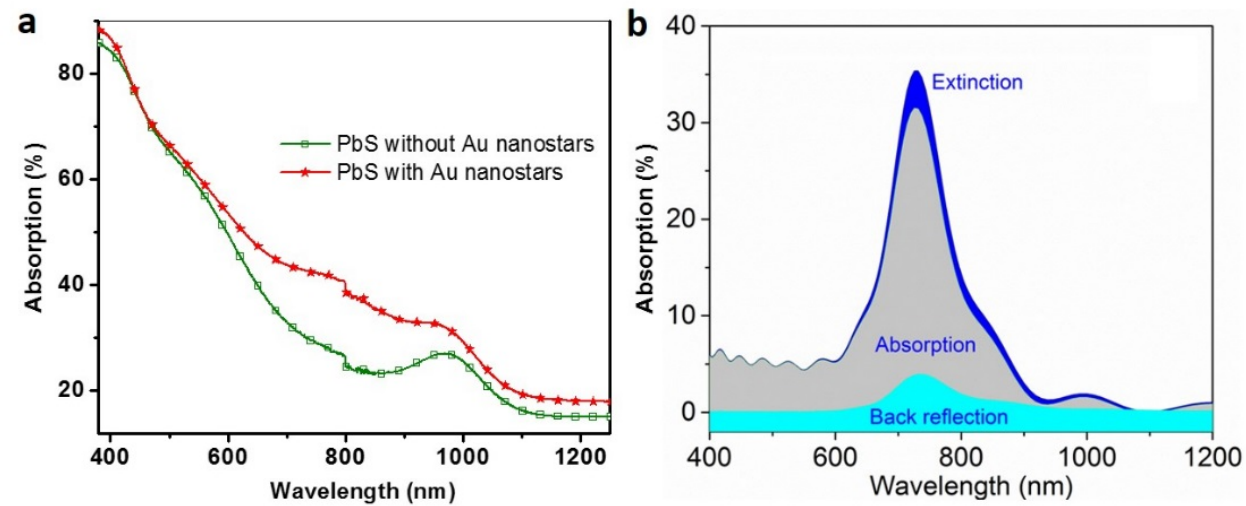

Figure 2.3. Solar cell and plasmon input parameters for optimal spatial position calculations. a, Experimental absorption of $300 \mathrm{~nm}$ thick PbS film with and without Au nanostars. b, Reflection and absorption ratio of a single Au nanostar calculated by FDTD simulations for one nanostar per 100 by $100 \mathrm{~nm}$ area. Reproduced with permission from Reference 14.

a

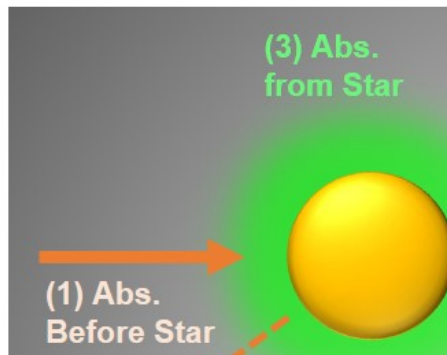

\section{(2) Abs. from Reflection}

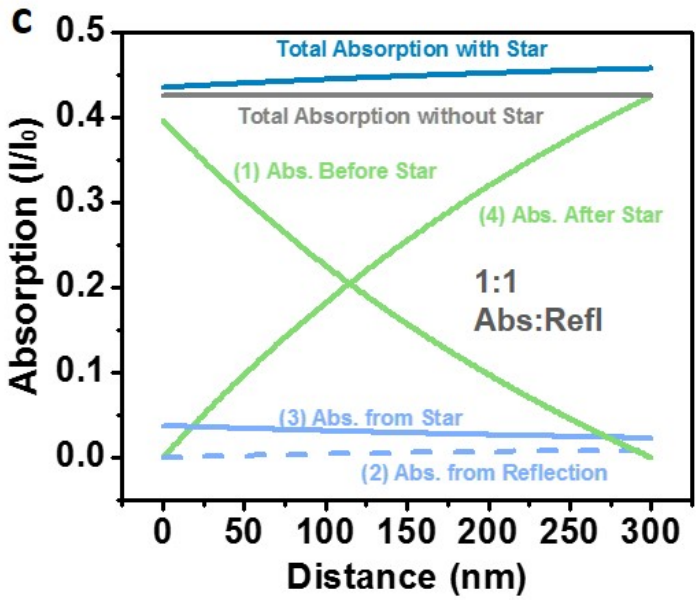

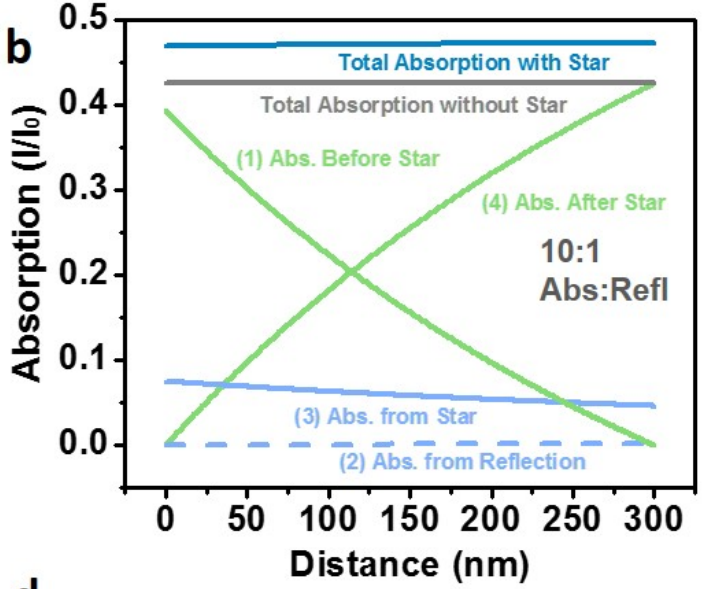

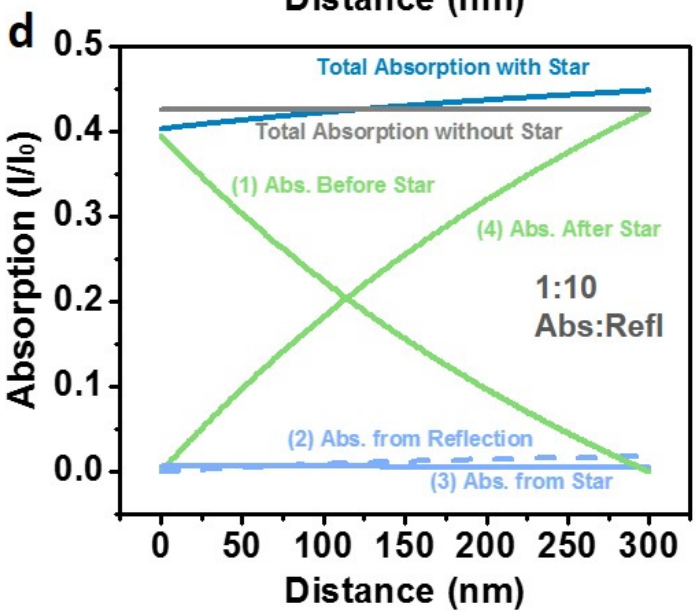

Figure 2.4. Balance of scattering versus absorption in light trapping. a, the four possible interaction pathways of the plasmon are calculated versus the position of the nanoparticle in a $300 \mathrm{~nm}$ QD film with paramaters taken from Ref. 14 for b, a 10:1 absorption to reflection rato, c, a 1:1 absorption to reflection ratio, d, and a 1:10 absorption:reflection ratio. 
The optimization problem is addressed in Figure 2.3 and 2.4 for the cases of plasmonic nanoparticles which primarily scatter, absorb, or have a balance of scattering and absorption. The overall increase in absorption was found by tabulating i) the light absorbed before the metal nanoparticle, ii) the light absorbed by reflection from the front of the metal nanoparticle, iii) the light transferred from metal-to-semiconductor by the near field (Chapter 4), and iv) the absorption of light in the semiconductor after the plasmon.

To verify the model, the parameters of a plasmon-enhanced QD solar cell fabricated by collaborators were used. ${ }^{14}$ This device was representative of typical spin-coated solar cells based on inorganic and organic light absorbing layers, such as a thickness of $300 \mathrm{~nm}$ and light absorption coefficient as measured in Figure 2.3. The plasmon's scattering and absorption cross section were modeled using finite difference time domain (FDTD) simulations of the gold nanostars commonly used in light trapping. The total increase in light absorption was integrated over the AM1.5G solar spectrum for each possible position within the 300 nm QD layer, indicating the optimal position and expected enhancement for a loading of one metal nanoparticles per 100 by $100 \mathrm{~nm}$ area. The nanostars have an absorption/reflection balance of 10:1, and the optimal location was found to be at the back of the QD layer (Figure 2.4b), wherein the small percentage of back-reflected light could lead to the largest percentage of re-absorption. An overall photoconversion enhancement of $10 \%$ was calculated, consistent with the experimental enhancement of $16 \%$ given the discrepancy in loading between the experiment and the 2D layer used in the simulations, as well as neglect of the effects of localized charge creation in the semiconductor near the active layer. ${ }^{14}$

In Figure 2.4b the absorption to scattering ratio is roughly 10:1. Next, the plasmonic nanoparticle's absorption scattering ratio was changed to $1: 1$ and 1:10 while keeping the total extinction cross section the same (Figure 2.4c and 2.4d). For the case of 1:10 absorption to scattering, the overall enhancement was actually lowered to $\sim 5 \%$ compared to the 10:1 absorption to scattering case. Similarly, for the case of 1:1 absorption to scattering the enhancement dropped to $\sim 8 \%$. In both cases, however, the optimal location remained at the back of the solar cell where back-reflected light could be most absorbed.

These basic calculations reveal an interesting insight when planning a light-trapping geometry. Unless the semiconductor's absorption cross section is large enough that for a few hundred nanometer active layer $50 \%$ of light is absorbed on the first pass and 50\% of light re-absorbed on the second pass, light trapping with scattering is not as efficient as light trapping with near-field coupling. This is because the reflected light is usually only partially absorbed on the second pass, limiting the enhancement due to the semiconductor's weak absorptionc cross section per unit thickness. When the plasmon absorbs light in the near field then transfers it to the semiconductor, the carrier creation is localized within the plasmon's near field, and the re-absorption length is no longer critical. In this case the plasmon's absorption cross section is what dictates absorption per unit thickness, leading to increases in light trapping without increases in semiconductor thickness. Therefore, unless multiple scattering pathways are possible, the best plasmonic light trapping geometry actually has the plasmon dominantly absorbing instead of scattering light, but still can utilize a weak reflection by placing the metal nanoparticles at the back of the semiconductor active layer. The same conclusions will hold true for plasmonically patterned back-reflectors, indicating that a back-reflector is better patterned for light trapping in the near-field than far-field reflection.

\subsection{SPP: High Surface Area, High Light Trapping Geometry}

For photovoltaics plasmonic light trapping can be optimized as shown in Section 2.2. In most solar cells the light absorption spectral range is optimized by the semiconductor's intrinsic band gap and the semiconductors are deposited in planar layers. The role of the plasmon is thus to allow the thinnest semiconductor possible to decrease charge recombination and transport losses without decreasing light 
absorption. ${ }^{15-19}$ For photocatalytic water splitting, enhancements by plasmonic light trapping is made more difficult by the band gap restrictions of the reaction $(1.8-2 \mathrm{eV})$ and the necessity of high surface areas to allow photocatalysis at the semiconductor-electrolyte interface. These difficulties are further compounded by the lack of semiconductors within the optimal band gap range with sufficient carrier lifetimes, mobility, and photostability. ${ }^{19-23}$

To overcome this issue a gold nanohole array back-electrode was developed. ${ }^{6}$ The metal nanohole array can support both surface plasmon polaritons (SPP) and localized surface plasmon resonance (LSPR) modes. ${ }^{24}$ The SPP resonance position is primarily controlled by the pitch of the nanohole array, whereas the LSPR resonance position is primarily tuned by the nanohole-diameter. ${ }^{25,26}$ During resonance, the LSPR localizes light in the near field, allowing an enhancement in the semiconductor above and near the band edge as discussed further in Chapter 4. At the SPP resonance, light is focused within the metal nanohole array, leading to increased transmission. This process, known as extraordinary optical transmission, is possible because the SPP modes on the metal/air and metal/dielectric interfaces on the two sides of the patterned metal film can couple. ${ }^{27-29}$ When the front air/metal interface is excited, the SPP mode localizes the light into the subwavelength polariton mode. This mode can then excite the back-side SPP mode, which then re-radiates into the dielectric because of the momentum matching of the nanohole array.

If semiconductor nanowires are then grown within the metal nanholes, during the SPP increased transmission light will be focused within the wires, with internal reflections trapping light like a miniature optical fiber. The metal nanohole array back-electrode could therefore allow a high surface area nanowire array to be created as a photoelectrode for water splitting, achieving sub-100 nm diffusion lengths without sacrificing absorption.

This concept was tested using hematite nanowires and a gold nanhole array as described in the following Section. ${ }^{6}$ Of possible semiconductor candidates, hematite $\left(\alpha-\mathrm{Fe}_{2} \mathrm{O}_{3}\right)$ was chosen because it is photostable and has a band gap of 1.9-2.2 eV. However, its application is hindered by several shortcomings: ${ }^{30-32}$ a short hole diffusion length (2-4 nm) compared to its light penetration depth ( 120 nm), short lifetime of charge carriers ( $<10 \mathrm{ps}$ ), and poor mobility of charge carriers $\left(<0.2 \mathrm{~cm}^{2} \cdot \mathrm{V}^{-1} \cdot \mathrm{s}^{-1}\right)$. Hematite can be engineered into a nanostructure to reduce the charge carrier diffusion distance to the electrode/electrolyte interface and to combat the short carrier lifetimes. However, the trade-off is that in order to satisfy the diffusion length, the structure must be much smaller than the optical absorption depth, limiting the amount of light harvested. By tuning a metal nanohole array to have a SPP resonance at energies above $2 \mathrm{eV}$ and a LSPR at energies below $2 \mathrm{eV}$, light absorption can be increased, and this trade-off is not necessary.

\subsubsection{Methods}

Synthesis of Au nanohole array pattern. The ordered Au nanohole array pattern was fabricated on the fluorine-doped tin oxide (FTO) substrate with nanosphere lithography. ${ }^{33,34}$ The FTO substrate was first treated with the acid solution to have a hydrophilic surface. Then $500 \mathrm{~nm}$ sized polystyrene (PS) monolayer was assembled on the FTO substrate. A $5 \mathrm{~nm}$ thick Ti, $45 \mathrm{~nm}$ thick Au, $5 \mathrm{~nm}$ thick Ti and $30 \mathrm{~nm}$ thick $\mathrm{SiO}_{2}$ layer were subsequently deposited on the FTO surface in sequence with an e-beam evaporation. Finally, the nanohole array pattern was obtained after lift-off of the PS spheres. For the disordered Au nanohole array, the same procedure was employed except that the PS template was dispersed on the FTO substrate by spinning coating instead of self-assembly on the water surface.

Growth of hematite nanorod array. Following a low-temperature hydrothermal method, ${ }^{35}$ the hematite nanorod array was grown on the bare FTO and on the Au hole array pattern respectively. Briefly, $0.15 \mathrm{M}$ $\mathrm{FeCl}_{3}$ and $1 \mathrm{M} \mathrm{NaNO}$ were dissolved in $40 \mathrm{~mL}$ of deionized (DI) water. Concentrated $\mathrm{HCl}$ was dropped 
into the solution to adjust the $\mathrm{pH}$ value to 1.5 . Pre-cleaned FTO and the Au nanohole array substrates were placed into the Teflon-lined autoclave. The autoclave was heated at $100{ }^{\circ} \mathrm{C}$ for different growth times to obtain different lengths of hematite nanorods. After completing washing with DI water and ethanol, the hematite nanorods were annealed in air at $650{ }^{\circ} \mathrm{C}$ for $20 \mathrm{~min}$.

Characterization. The morphology of the hematite nanorods and the Au nanohole array pattern were observed with a field-emission scanning electron microscope (FE-SEM, JEOL 7600F) and a transmission electron microscope (TEM, JEOL JEM 2100F), respectively. The crystal structure of hematite nanorods was characterized with a high-resolution TEM (HRTEM, JEOL JEM 2100F) and X-ray diffraction (XRD, X’ Pert Pro PW 3040-Pro, Panalytical Inc.) with $\mathrm{Cu} \mathrm{K} \alpha$ radiation. The light-absorption properties were measured by the diffuse-reflection mode with Shimadzu 2550 UV-Visible spectrometer equipped with an integrating sphere (UV 2401/2, Shimadzu). Absorption measurement was taken using an integrating sphere to reduce the effects of scattering of the nanorod array on the measurement. Both transmission and reflection were measured to calculate the absorption.

Photoelectrochemical Measurement. The PEC behavior of hematite was tested with a three-electrode configuration with a Gamry Reference $3000^{\mathrm{TM}}$ Potentiostat/Galvanostat/ZRA Instrument. The hematite nanorod array was employed as the working electrode, the Pt foil as the counter electrode, and $\mathrm{Ag} \mid \mathrm{AgCl}$ as the reference electrode. $1 \mathrm{M} \mathrm{NaOH}$ aqueous solution was used as electrolyte and buddle with $\mathrm{N}_{2}$ for 30 min prior to measurement. The light source employed in this study was a 300W Xe lamp with an AM 1.5G filter. All the PEC measurements were performed on at least three samples and each sample was tested at least three times.

Technical Details FDTD Simulations. To confirm the origin of the enhancement observed in the IPCE spectrum, FDTD simulations were run using the commercially available Optiwave software, similar to my previous work. ${ }^{36,37}$ The mode calculations were performed using Optimode. The simulation cell was constructed identically to the Au nanohole array (Fig. 2.6a), and the corresponding UV-Visible spectrum was matched to that of the Au nanohole array (Fig. 2.7a) to ensure consistency. The hematite was modeled using the full frequency dependent dielectric function, ${ }^{38} \mathrm{Au}$ and titanium using the data of $\mathrm{Palik}^{39}$ and $\mathrm{SiO}_{2}$ using a refractive index of 1.4. The reported values of bulk $\mathrm{Fe}_{2} \mathrm{O}_{3}$ vary depending on the synthesis procedure. ${ }^{40}$ For the simulation, an absorption coefficient of $5 \times 10^{4}$ at $500 \mathrm{~nm}$ was used. The resolution of the cell was $2 \mathrm{~nm}$ and the input wave was a Gaussian pulse, linearly polarized in the $Y$ direction. Periodic boundary conditions were used to replicate the periodic nanohole array.

The local field was examined verse excitation wavelength to confirm the nature of the plasmonic modes. The local field was normalized by that in the absence of the nanostructure, and was reported as $\left|\mathrm{E} / \mathrm{E}_{0}\right|^{2}$. The local field of the LSPR mode was determined by measuring the maximum at each excitation wavelength. If multiple maximums were present at different locations, the largest field enhancement was reported. A field enhancement was present at both SPP and LSPR modes. However, in the SPP, the field enhancement led to an increased transmission, masking the maximum field enhancement. Therefore, the field enhancement was only reported in the region of the LSPR where increased transmission was negligible.

Identification of SPP and LSPR modes. Two modes can exist in the metal nanohole array. The first is a surface plasmon polariton (SPP) which corresponds to an oscillating charge wave at the metal semiconductor interface (Figure 2.5). The SPP can only be excited by matching the momentum of the charge wave to the incident photon through the periodicity of the nanostructure. Just as the periodicity allows coupling to incident light, the SPP modes can re-couple to the far field on the opposite side of the nanohole array, leading to an enhanced transmission (Figure 2.5d). The localized surface plasmon resonance (LSPR) does not propagate, and instead is localized at the edges of the nanohole. The local charge 
oscillations lead to an intense local field at the interfaces of the metal/dielectric (Figure 2.5e). In the LSPR, the momentum matching comes from the curvature of the nanoscale shape, and is only dependent on the geometry of the nanohole and not on the periodicity of the array. Therefore an increase in absorption or transmission can easily be assigned to a SPP or LSPR mode by turning off the periodicity in the FDTD boundary conditions (Figure 2.5c).
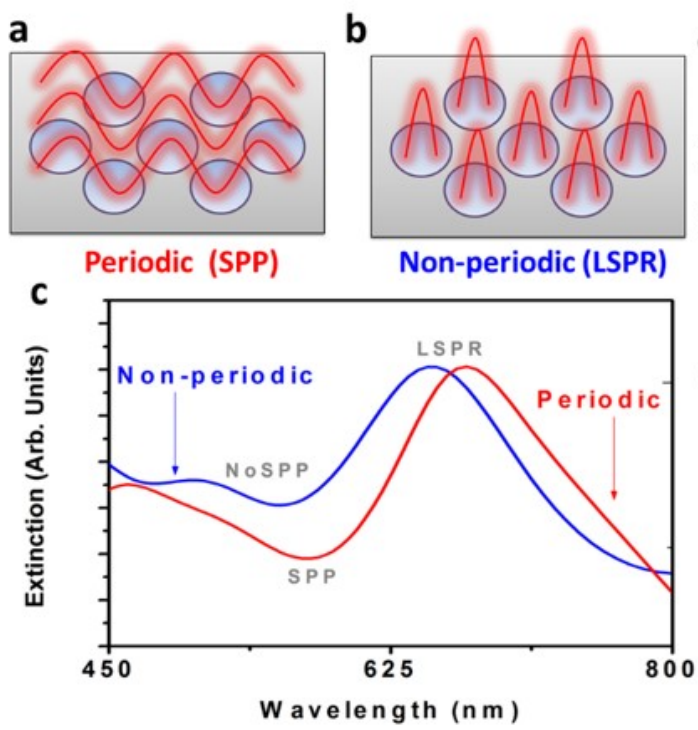

b

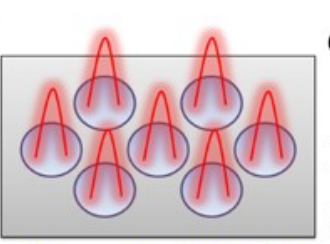

\section{)}
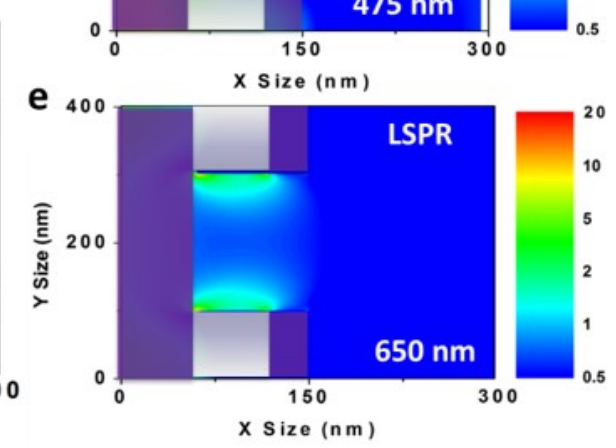

Figure 2.5. Comparison and separation of SPP and LSPR modes. a, SPP are travelling charge oscillation waves that form due to coupling of incident light with the periodicity of the nanohole array; $\mathbf{b}$, LSPR is isolated in each hole and is present regardless of the periodicity; c, The SPP can lead to an enhanced transmission while the LSPR leads to an increased absorption. When the periodicity of the cell is removed, the SPP and increased transmission no longer exists; d, The local plasmonic field in the SPP can reradiate the incident light, leading to an increased transmission; e, The LSPR local field enhancement is centered at the edges of the hole and the interface between the metal and dielectric.

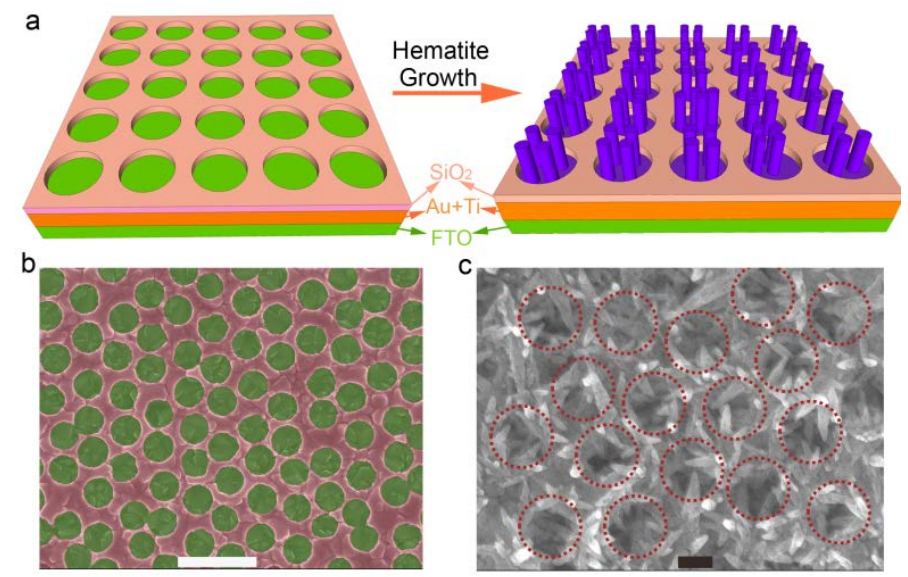

Figure 2.6. Geometrical and microscopic structures for plasmonic design. a, Scheme for the growth of hematite nanorod array on Au nanohole array with FTO substrates exposed. SEM images of the Au nanohole array $\mathbf{b}$, without and $\mathbf{c}$, with the hematite nanorods. Scale bars: $1 \mu \mathrm{m}$ in $\mathbf{b}$ and $200 \mathrm{~nm}$ in $\mathbf{c}$.

\subsubsection{Results}

At first, the long-range ordered plasmonic Au nanohole array pattern was fabricated using nanosphere 
lithography on a fluorine-doped tin oxide (FTO) substrate. The Au nanoholes were $85 \mathrm{~nm}$ high and $350 \mathrm{~nm}$ in a diameter (Fig. 2.6b). The center-to-center distance between adjacent holes was around $490 \mathrm{~nm}$. The hole width, pitch, and the Au film thickness were chosen to enable the SPP and LSPR modes at the wavelengths below and above the band gap of hematite, respectively. The hematite nanorods were grown on the Au nanohole array pattern with a hydrothermal method, followed by annealing at $650{ }^{\circ} \mathrm{C}$ (Fig. 2.6a). The hematite nanorods were grown inside the hole regions. Hematite displayed the same nanorod morphology on both the bare FTO and the Au nanohole array pattern (Fig. 2.6c). Transmission electron microscopy (TEM), higher resolution transmission electron microscopy (HRTEM) and x-ray diffraction (XRD) confirmed the formation of hematite, see Reference 6 for full characterization.
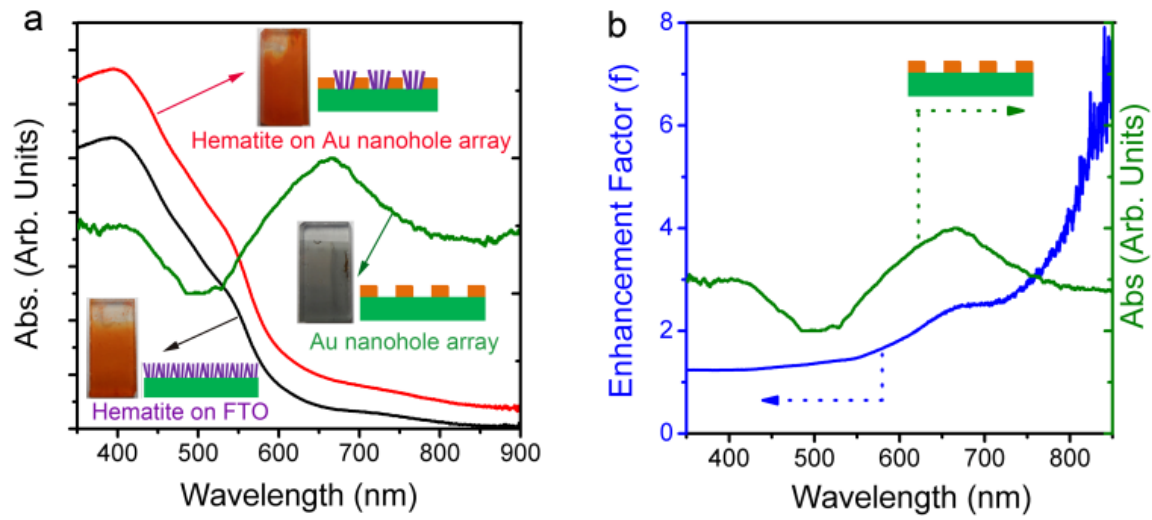

Figure 2.7. Plasmon enhanced light absorption. a, UV-Visible absorption spectra for $150 \mathrm{~nm}$ long hematite nanorods on the bare FTO and on the Au nanohole array pattern, respectively. The insets show the corresponding digital pictures of photoanodes. b, Absorption enhancement for the hematite nanorods on the Au nanohole array pattern with the background (hematite on FTO) subtracted.

The UV-Visible absorption spectrum of the bare Au nanohole array pattern had a strong absorption peak centered at $650 \mathrm{~nm}$ and an enhanced transmission peak at $475 \mathrm{~nm}$ compared to an un-patterned gold film of the same thickness (Figure 2.7a). The hematite nanorods grown on the bare FTO substrates absorbed light with a tail up to $700 \mathrm{~nm}$. The band gap was determined to be $2.0 \mathrm{eV}$ using a tangent line to the absorption edge (Fig. 2.7a). The additional exponentially decaying absorption tail was attributed to an Urbach tail formed by defect states in the hematite. When the hematite nanorods were grown on the Au nanohole array, an increase in absorption across the entire spectral range was seen compared to hematite on bare FTO. After division of the hematite background from the hematite/nanohole array sample, an absorption band centered at around $650 \mathrm{~nm}$ was observed (Fig. 2.7b), which matched the LSPR peak for the bare Au nanohole array pattern. The enhancement factor represents the division of the two UV-Visible spectra (not the percentage change). The high tail after $750 \mathrm{~nm}$ is an artifact of the division process due to the low absorption coefficient of hematite at higher wavelengths (Fig. 2.7a). The nanohole array transmission peak centered at $475 \mathrm{~nm}$ was not as easily seen in the hematite/nanohole array sample as the absorption peak at $650 \mathrm{~nm}$. However, the absorption was seen to be enhanced proportional to the band edge of hematite (Fig. 2.7a). Since the hematite nanorods on the Au nanohole array pattern had an equal or lower density when compared to those grown on bare FTO, the SPP must be responsible for the increase in the absorption of light in hematite above the band gap.

The PEC performance was measured in $1.0 \mathrm{M} \mathrm{NaOH}$ electrolyte using a three-electrode cell for the hematite nanorods on both the bare FTO and the Au nanohole array pattern. For the PEC measurements, the photoanode was backside illuminated by $100 \mathrm{~mW} / \mathrm{cm}^{2}$ of simulated solar light from a $300 \mathrm{~W}$ Xenon 
lamp through an AM 1.5G solar simulator filter. The current density in Fig. 2.8a was calculated based on the whole geometric area of photoanode instead of the effectively electro-active area (hole regions) on the photoanode. The photocurrent was significantly enhanced for the $150 \mathrm{~nm}$ long hematite nanorods grown on the gold nanohole array pattern when compared to hematite grown on bare FTO (Fig. 2.8a), with an enhancement factor as high as 10 at a bias of $0.23 \mathrm{~V}$ (vs. Ag|AgCl) under simulated solar full spectrum. The onset potential of the photocurrent remained the same for the hematite nanorods on both the bare FTO and the Au nanohole array pattern, which indicates that the Au nanohole array pattern had negligible influence on the surface catalysis process at the semiconductor-electrolyte interface. Hence the significant enhancement of the photocurrent must be due to the LSPR and/or SPP modes in the gold nanohole array.
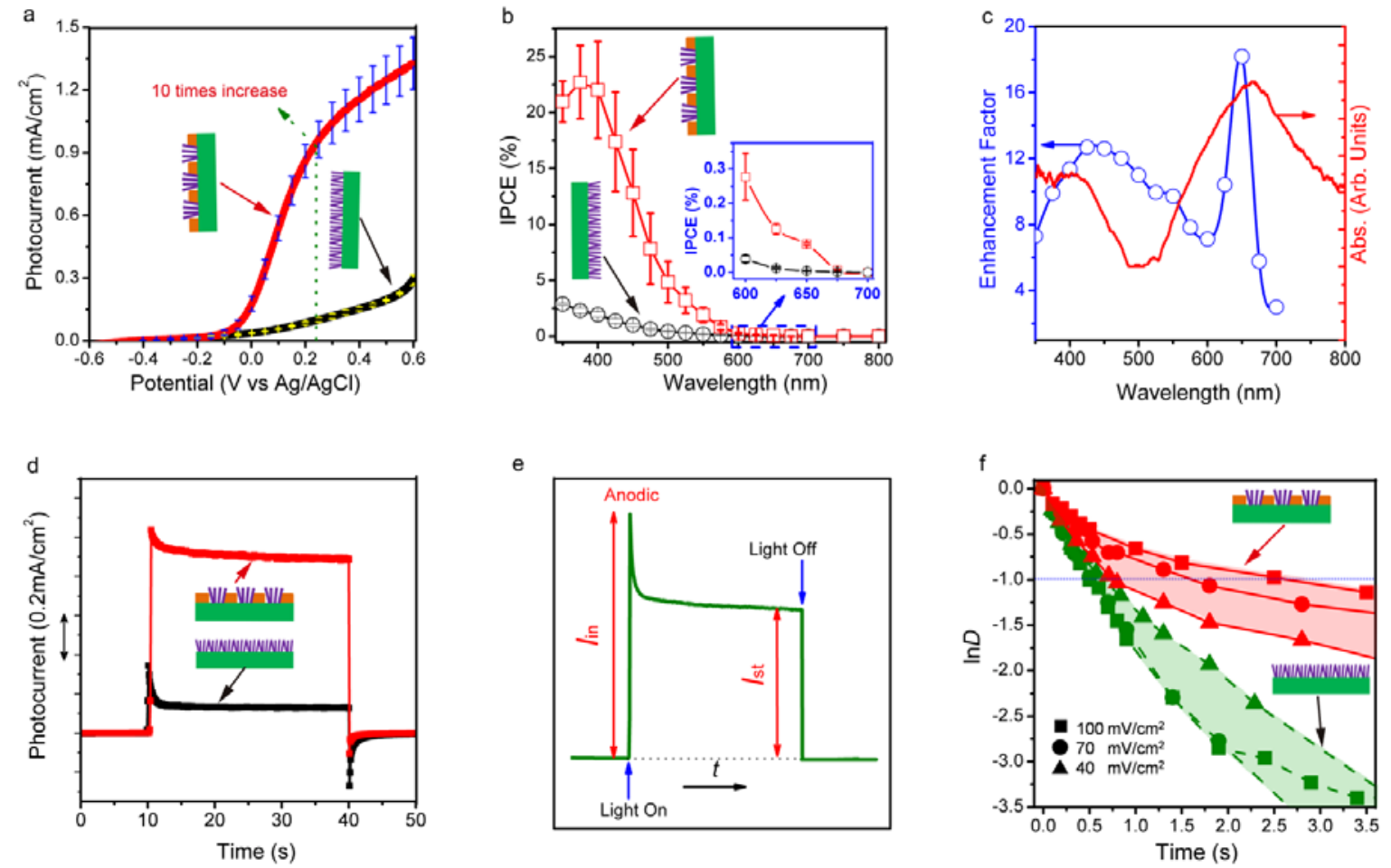

Figure 2.7. PEC performance of the hematite nanorod arrays on the FTO and on the Au nanohole array pattern. a, JV curves under the illumination of AM 1.5G full-spectrum solar light with a power density of $100 \mathrm{~mW} / \mathrm{cm}^{2}$. b, IPCE spectra; the insert is the IPCE in 600-700 nm range. (c) The photocurrent enhancement factor as a function of the wavelength. d-f, Anodic photocurrent dynamics of photoelectrodes at an applied bias $0.23 \mathrm{~V}$ vs. Ag|AgCl. e, The scheme for the calculation of the transient dynamics constant. f. Anodic transient dynamics under AM 1.5G fullspectrum solar light with different irradiation intensities.

The incident photon-to-electron efficiency (IPCE) spectrum was measured to elucidate the correlation of the LSPR and SPP modes with the enhanced photocurrent (Fig. 2.7b). The hematite nanorods on the Au nanohole pattern showed a substantially enhanced IPCE in the wavelength range from $325 \mathrm{~nm}$ to $700 \mathrm{~nm}$ when compared to hematite on bare FTO. The IPCE at $425 \mathrm{~nm}$ was $1.37 \%$ and $17.4 \%$ for the hematite nanorods on the bare FTO and on the long-range ordered Au hole array, respectively. An IPCE enhancement spectrum was obtained by dividing the two corresponding IPCE spectra (Fig. 2.7c), revealing two distinct enhancement peaks of 13 times enhancement at $425 \mathrm{~nm}$ and 18 times enhancement at $650 \mathrm{~nm}$. These positions correlate well with the enhanced transmission and absorption of the Au nanohole array, which finite difference time domain (FDTD) simulations confirmed as being caused by SPP and LSPR modes, respectively (Figure 2.5 ). 
Charge recombination is typically a large bottleneck in the hematite photoanode. Photocurrent transient measurement was therefore performed to assess if the metal nanohole array suppresses the charge recombination rate. To quantitatively determine the charge recombination behavior, a normalized parameter $(D)$ is introduced:

$$
D=\left(I_{\mathrm{t}}-I_{\mathrm{st}}\right) /\left(I_{\mathrm{in}}-I_{\mathrm{st}}\right)
$$

Where $I_{\mathrm{t}}, I_{\mathrm{st}}$ and $I_{\text {in }}$ are the time-dependent, steady-state and initial photocurrent, respectively, as shown in Fig. $2.7 \mathrm{e} .{ }^{41}$ The transient time constant $(\tau)$ is defined as the time when $\ln D=-1$ in the normalized plots of $\ln D$ - $t$ (Fig. 2.7f), which reflects the general behavior of charge recombination and lifetime of the charge carriers. $\tau$ was estimated to be $2.51 \mathrm{~s}$ for the hematite on the long-range ordered Au nanohole array pattern under $100 \mathrm{~mW} / \mathrm{cm}^{2}$ of simulated solar light, which was five times that for hematite without the Au nanohole array pattern $(0.53 \mathrm{~s})$, indicating suppressed charge recombination.

The increase in the recombination lifetime was not due to surface chemistry or bulk trapping states since the onset potential (in Fig. 2.7a) did not change with the incorporation of hematite with the plasmonic Au nanohole pattern. Rather, the intense localized electromagnetic field gradient created by the plasmonic Au nanohole pattern must lead to the lower charge recombination rate. To investigate this effect, the intensity of the incident field was modulated using the full-spectrum neutral density filters (Figure 2.7f). The normalized photocurrents demonstrate that the decay was altered when varying the incident light intensity for hematite on the long-range ordered Au nanohole array pattern, while no change was observed when varying the light intensity for hematite on the bare FTO. As expected, the lifetime of the carriers increased with an increase in the irradiation intensity for hematite on the long-range ordered Au nanohole array pattern since the local electromagnetic field gradient was proportional to the light intensity. However, hematite on the bare FTO had the same transient time constant within the error scale, independent of the incident intensity, which indicates that the localized electromagnetic field near the Au nanoholes must cause a difference in the carrier lifetime.
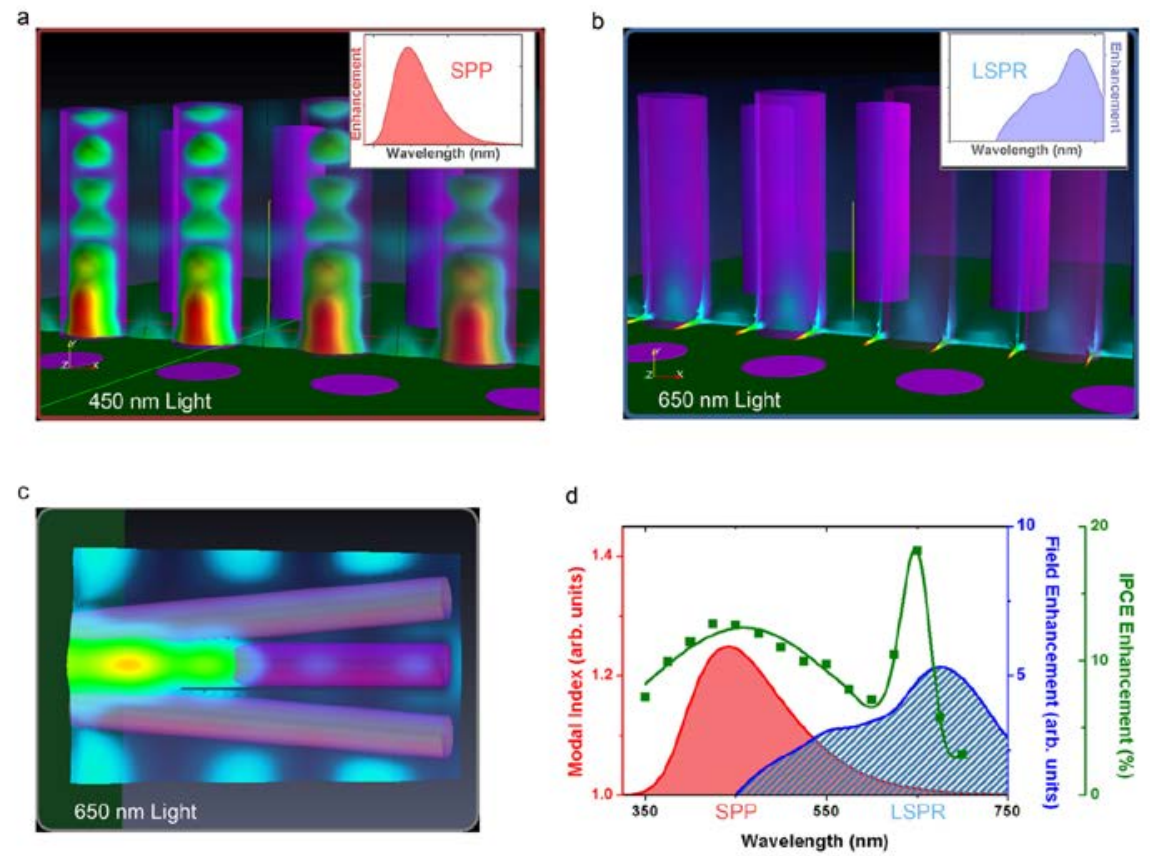

Figure 2.8. Finite difference time domain simulations of the Au nanohole array with hematite nanorods. a, At wavelengths corresponding to an increased transmission in the Au nanohole array, the SPP led to trapping of incident light as waveguided modes in the hematite nanorod. b, At wavelengths corresponding to the LSPR no waveguiding is 
seen, rather the incident field is localized at the edges of the hole. c, The same waveguiding exists in a simulation more similar to experimental samples. d, The waveguiding efficiency and local field enhancement are seen to accurately explain the experimental IPCE.

\subsubsection{Discussion}

The SPP and LSPR plasmonic fields combine to create an increase in photoconversion efficiency and an increase in carrier lifetime. The different origins of the SPP and LSPR modes, however, lead to two distinct forms of enhancement to the solar energy conversion efficiency which are complimentary across the solar spectrum. In the 300-500 nm region, the periodicity of the nanohole array allows the incident light to couple to the SPP mode, which is a travelling charge density wave in the metal film (Figure 2.5). The travelling SPP mode can reradiate into the far field when interrupted by a nanohole, increasing the optical transmission at that wavelength. Since the nanoholes are filled with the hematite nanorods, the increased photon flux from the extraordinary optical transmission will be directly launched into the nanorod instead of being re-radiated from the nanohole. The high contrast in dielectric constant between the semiconductor and the surrounding environment, coupled with the subwavelength nature of the plasmon emission, will therefore allow the SPP to excite a guided mode in the nanorod, increasing absorption in hematite.

This behavior can be seen in a simple FDTD model, consisting of a gold nanohole array identical to experiment but with a single, uniform hematite nanorod grown in each nanohole (Fig. 2.8a). The resulting normalized electromagnetic (EM) field distribution (Fig. 2.8a) shows that at the SPP resonance (450 nm), the incident light is concentrated in the metal nanohole, which subsequently launches a guided optical mode in the hematite nanorod. The guided nature of the optical mode is revealed by the well-defined nodes in the averaged field (Fig. 2.8a). The mode can be further shown in a cross cut of the nanowire in Figure 2.9, which shows that it is distributed mainly in the core of the nanowire but extends into and past the surface as the wavelength increases and the waveguiding efficiency decreases. Even with multiple nanorods in the metal nanohole, waveguiding is still present, creating an optical mode split between each nanorod (Fig. 2.8c).

The wave-guiding enhancement versus wavelength can be further quantified using mode solver calculations for the realistic, $\sim 120 \mathrm{~nm}$ hematite nanorods. The results are displayed in terms of the modal index in Fig. 2.8d. For the single mode examined, the higher the modal index the more tightly bound the waveguiding can be thought of as in the hematite core (Figure 2.9c), with a modal index of one corresponding to the absence of waveguiding. The waveguiding efficiency verse the wavelength matches excellently to the $425 \mathrm{~nm}$ enhancement peak in the IPCE spectrum (Fig. 2.8d). This confirms that the first enhancement in the IPCE comes from the SPP, which launches a guided optical mode in the nanorod, turning the nanorod into a miniature "optical fiber" and increasing the light absorption at the energies above the band edge of hematite.

The SPP leads to an enhanced transmission in the 300-500 nm range, whereas the LSPR mode induces an increased absorption in the 550-750 nm range. LSPR does not create extraordinary optical transmission. Instead, the absorption of the LSPR creates an intense local field at the walls of the nanohole in the metal film. The energy absorbed by the LSPR can be transferred to the semiconductor at the energies below the band edge through hot electron transfer (also known as direct electron transfer or DET and discussed in Chapter 3) and/or PIRET as discussed in Chapter 4, allowing an increase in the IPCE. FDTD simulation of the simple model of a uniform hematite nanorod in the metal nanohole array was repeated at the wavelengths close to the LSPR absorption $(650 \mathrm{~nm})$. The LSPR created a sharp field enhancement at the metal/hematite interface, but little light was transmitted into the hematite nanorod (Fig. 2.8b). When the peak field enhancement $\left|\mathrm{E} / \mathrm{E}_{0}\right|^{2}$ was extracted verse wavelength, it was seen to overlap with the hematite band edge and closely correlate with the second enhancement peak in the IPCE, which confirms that a 
plasmonic energy transfer mechanism must be responsible for the enhancement (Fig. 2.8d).
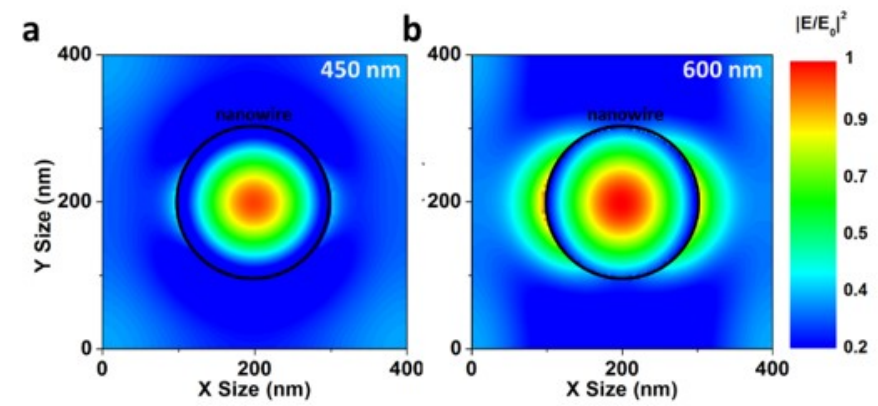

C
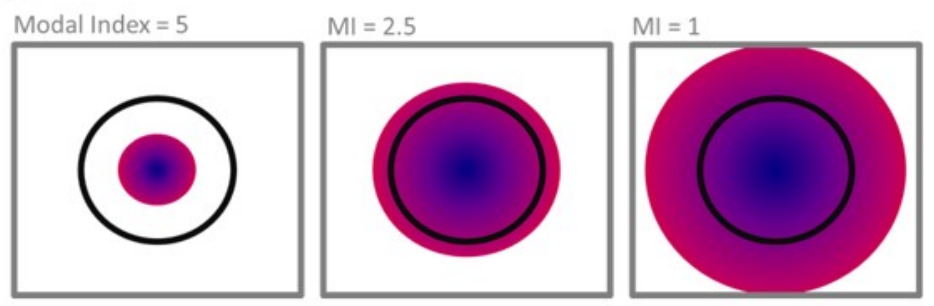

Figure 2.9. Technical Details of Mode Calculations. a, Normalized mode intensity of the hematite nanorod in the ordered Au nanohole array. At $450 \mathrm{~nm}$, the mode is mainly centered in the middle of the hematite nanorod with minimal coupling to the surface. $\mathbf{b}$, As the wavelength increases, the waveguiding efficiency decreases quickly, and the mode is located more at the surface of the nanorod and into the local environment. c,The modal index changes from the refractive index of the semiconductor to the refractive index of air as the waveguiding efficiency goes from localized to nonexistent. The mode calculation was run in Optimode. The $120 \mathrm{~nm}$ diameter fibers were simulated using the full refractive index of hematite. The modal index was solved for at each wavelength using a linearly polarized solver in 2D. The modal index can be thought of as representing how efficiently the incident light is guided. A high modal index means all incident light is trapped in a propagating mode, whereas a modal index of one means no waveguiding is present.
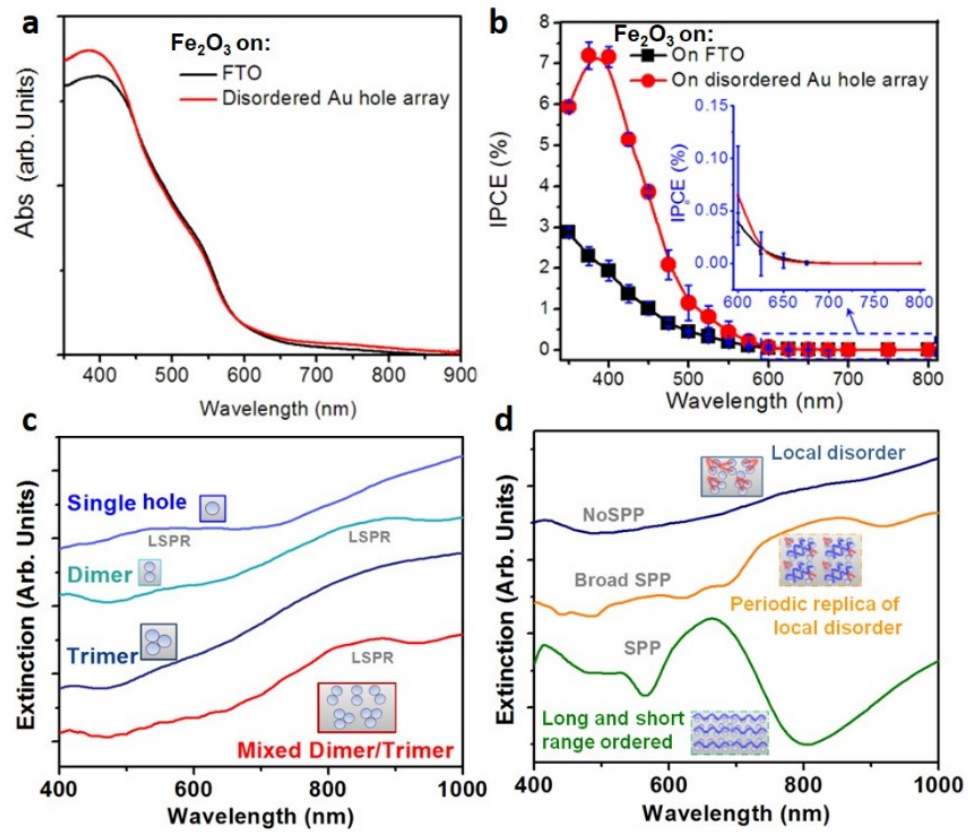

Figure 2.10. Effect of disorder on plasmonic enhancement. a, Light absorption of hematite nanowires on FTO and on the disordered gold nanhole array. b, IPCE of hematite nanowires on FTO and on the disordered gold nanhole array. c, Single holes have a very weak LSPR (hole size: $350 \mathrm{~nm}$ ). The dimer-holes display a red-shifted LSPR band at 
around $800 \mathrm{~nm}$. The trimer-holes have a broadened and red-shifted LSPR band. The random mixture of dimer and trimer holes in a small area shows a broad LSPR band. (The curves are offset for clarity). d, For a local disordered unit cell containing two single holes, three dimers, and two trimer holes in a small area, a red-shifted LSPR peak is present while the SPP mode is absent due to the lack of periodicity over a large-area. A large-area periodic replica of the local disordered unit cell shows the weak SPP modes and a red-shifted LSPR peak. A large-area long-range ordered hole array exhibits the strong SPP mode and a blue-shifted LSPR peak due to the absence of inter-hole interaction present with dimer and trimer hole pairs.

In the present work, hematite is not in direct physical contact with the plasmonic Au. DET therefore cannot be responsible for the LSPR-based IPCE enhancement. However, the LSPR absorption band does overlap with the absorption band of hematite, which makes PIRET the most likely plasmonic energy transfer mechanism. ${ }^{43,44}$ The presence of PIRET was confirmed by conduct similar measurement with a $\mathrm{TiO}_{2}$ nanorod array on the Au hole array pattern. This control experiment showed no enhancement in the IPCE at $650 \mathrm{~nm}$ (data not shown). This is not surprising because the absorbance band of $\mathrm{TiO}_{2}$ has no spectral overlap with the LSPR absorbance peak of the Au hole array, which makes PIRET impossible. This confirms that PIRET transfers the energy in the LSPR field to hematite, allowing enhancement in the IPCE at the wavelengths below the band edge of hematite (Fig. 2.8d).

The periodicity of the plasmonic pattern can affect the plasmonic properties of the metal nanostructures. ${ }^{44,45}$ As a control sample, a disordered Au hole array pattern was fabricated with the same hole size but without long-range order (Figure 2.10). In this disordered hole array, the distance between the holes were varied and even dimer and trimer hole pairs appeared in a local area. The hematite nanorod array was then grown on the disordered Au hole array. It can be seen from the UV-Visible absorption spectra (Figure 2.10a) that the SPP transmission peak became weakened and the LSPR peak red-shifted to $800 \mathrm{~nm}$ for the disordered Au hole array without hematite nanorods. The red-shift of LSPR peak is due to the coupling between the now aggregated dimer and trimer hole pairs in the local area. Even though the hole array is highly disordered, a transmission enhancement is still possible due to the periodicity of local disorder domains in the hole array, as shown by FDTD simulation (Figure 2.10c and 2.10d). Therefore, the photocurrent of the hematite nanorods array on the disordered Au hole array exhibited only 2.8 times enhancement of that of hematite on the bare FTO substrate at a bias of $0.23 \mathrm{~V}$ (vs. Ag|AgCl) under simulated solar full spectrum. The enhancement effect of the disordered Au hole array was smaller than the longrange ordered hole array, which was expected from a decrease in the SPP transmission peak and from the red-shift of the LSPR peak.

The disordered hole array allows further confirmation of the separate LSPR and SPP based enhancements. It is worth noting that red-shift of the LSPR band led to no spectral overlap between the absorption edge of hematite and the LSPR absorbance band of the disordered Au hole array, preventing PIRET. The IPCE data reflected this change with no enhancement at the wavelengths above the band edge of hematite (Figure 2.10b). The weak SPP mode in the disordered pattern allowed a small enhancement at the SPP transmission peak (IPCE at $425 \mathrm{~nm}=5.18 \%$ ). As the long-range order increases, the SPP transmission becomes stronger, and the IPCE enhancement increases in strength but does not change spectrally. Additionally, in the long-range ordered pattern, the LSPR band can overlap with the absorption edge of hematite, enabling the PIRET process.

\subsection{Chapter Summary}

In this Chapter, it was seen how to achieve optimal light trapping for both photovoltaic and photocatalysis. In Section 2.2, it was shown that for $<300 \mathrm{~nm}$ semiconductor films, the plasmon's near field 
is better for use in light trapping than the plasmon's reflection because of poor re-absorption of backscattered light. In Section 2.3, a broad-wavelength light-harvesting enhancement that spanned the full UVNIR range was realized in the hematite nanorod-Au nanohole array pattern architecture. The extraordinary transmission of the SPP modes was used to concentrate the incident light at the energies above the band edge of hematite. The nanorods acted as miniature "fiber optics", creating the confined modes, which trapped the incident light and enhanced the light absorption. The intense local field enhancement of the LSPR overlapped with the absorption band edge of hematite, enhancing solar energy harvesting at the energies below the band edge through the PIRET mechanism. As a result, the photonic (13 times enhancement at $425 \mathrm{~nm}$ ) and the plasmonic energy transfer enhancement (18 times enhancement at 650 $\mathrm{nm}$ ) were revealed in the IPCE spectrum. Finally, the intense local field gradient increased the carrier lifetime in hematite. This metal/semiconductor heterostructure enhanced both the spectral utilization and the carrier lifetime in the semiconductor in an easy to manufacture geometry, making it ideal for plasmon-

enhanced solar energy conversion. This light trapping geometry is equally applicable for photovoltaics or photocatalysis, showing many advantages compared to the simpler geometry of using only a back-scattering layer.

\subsection{References}

1. Catchpole, K. R.; Polman, A. Plasmonic solar cells. Opt. Express 2008, 16, 21793.

2. Schuller, J. A.; Barnard, E. S.; Cai, W.; Jun, Y. C.; White, J. S.; Brongersma, M. L. Nat. Mater. 2010, 9, 193-204.

3. Pala, R. A.; Liu, J. S. Q.; Barnard, E. S.; Askarov, D.; Garnett, E. C.; Fan, S.; Brongersma, M. L. Nat. Commun. 2013, 4, 1643.

4. Aydin, K.; Ferry, V. E.; Briggs, R. M.; Atwater, H. A. Nat. Commun. 2011, 2, 517.

5. Ferry, V. E.; Verschuuren, M. A.; Li, H. B. T.; Verhagen, E.; Walters, R. J.; Schropp, R. E. I.; Atwater, H. A.; Polman, A. Opt. Express 2010, 18, A237.

6. Li, J.; Cushing, S. K.; Zheng, P.; Meng, F.; Chu, D.; Wu, N. Nat. Commun. 2013, 4, 2651.

7. Kim, S.-S.; Na, S.-I.; Jo, J.; Kim, D.-Y.; Nah, Y.-C. Appl. Phys. Lett. 2008, 93, 073307.

8. Tan, H.; Santbergen, R.; Smets, A. H. M.; Zeman, M. Nano Lett. 2012, 12, 4070-4076.

9. Atwater, H. A.; Polman, A. Nat. Mater. 2010, 9, 205-213.

10. Link, S.; El-Sayed, M. A. Int. Rev. Phys. Chem. 2000, 19, 409-453.

11. Sönnichsen, C.; Franzl, T.; Wilk, T.; von Plessen, G.; Feldmann, J.; Wilson, O.; Mulvaney, P. Phys. Rev. Lett. 2002, 88 (7), 077402.

12. Link, S.; El-Sayed, M. A. J. Phys. Chem. B 1999, 103, 4212-4217.

13. Peña-Rodríguez, O.; Pérez, P. P. G.; Pal, U. Int. J. Spectrosc. 2011, 583743, 1-10.

14. Gonfa, B. A.; Kim, M. R.; Zheng, P.; Cushing, S. K.; Wu, N.; Khakani, M. A. E.; Ma, D.; In Submission 2015.

15. Formal, F. L.; Tetreault, N.; Cornuz, M.; Moehl, T.; Grätzel, M.; Sivula, K. Chem. Sci. 2011, 2, 737-743.

16. Formal, F. L.; Grätzel, M.; Sivula, K. Funct. Mater. 2010, 10, 1099-1107.

17. Meng, F.; et al. ACS Catal. 2013, 3, 746-751.

18. Li, J. T.; Meng, F.; Suri, S.; Ding, W.; Huang, F. Q.; Wu, N. Q. Chem. Commun. 2012, 48, 82138215.

19. Klahr, B. M., Gimenez, S., Fabregat-Santiago, F., Hamann, T. \& Bisquert, J. J. Am. Chem. Soc. 
2012, 134, 4294-4302.

20. Wu, N. Q. et al. J. Am. Chem. Soc. 2010, 132, 6679-6685.

21. Wang, J. et al. J. Am. Chem. Soc. 2009, 131, 12290-12297.

22. Lin, Y. et al. J. Am. Chem. Soc. 2012, 134, 5508-5511.

23. Sivula, K.; Formal, F. L.; Grätzel, M. ChemSusChem 2011, 4, 432-449.

24. Schwind, M.; Kasemo, B.; Zoric, I. Nano Lett. 2013, 13, 1743-1750.

25. Yang, Z. L.; Li, Q.H.; Renb, B.; Tianb, Z.Q. Chem. Commun. 2011, 47, 3909-3911.

26. Zheng, P.; Cushing, S. K.; Suri, S.; Wu, N. Phys. Chem. Chem. Phys. 2015, DOI: 10.1039/C4CP05291A

27. Ebbesen, T. W.; Lezec, H. J.; Ghaemi, H. F.; Thio, T.; Wolff, P. A. Nature 1998, 391, 667-669.

28. Ekinci, Y.; Solak, H. H.; David, C. Optics Lett. 2007, 32, 172-174.

29. van Beijnum, F.; Retif, C.; Smiet, C. B.; Liu, H.; Lalanne, P.; van Exter, M. P. Nature 2012, 492, 411-414.

30. Katz, M. J.; Riha, S. C.; Jeong, N. C.; Martinson, A. B. F.; Farha, O. K.; Hupp, J. T. Coordination Chem. Rev. 2012, 256, 2521-2529.

31. Mayer, M. T.; Du, C.; Wang, D. J. Am. Chem. Soc. 2012, 134, 12406-12409.

32. Chernomordik, B. D. et al. Nanotechnology 2012, 23, 194009.

33. Li, H.; Wu, N. Q. Nanotechnology 2008, 19, 275301.

34. Li, H.; Low, J.; Brown, K. S.; Wu, N. Q. IEEE Sensors J. 2008, 8, 880-884.

35. Vayssieres, L.; Beermann, N.; Lindquist, S. E.; Hagfeldt, A. Chem. Mater. 2001, 13, 233-235.

36. Li, M. et al. Nanotechnology 2012, 23, 115501.

37. Cushing, S. K.; Hornak, L. A.; Lankford, J.; Liu, Y.; Wu, N. Q. Appl. Phys. A 2011, 103, 955-958.

38. Galuza, A. I.; Eremenko, V. V.; Kirichenko, A. P. Sov. Phys. Solid State 1979, 21, 654-656.

39. Palik, E. D. Handbook of Optical Constants; Academic Press: Waltham, 1991.

40. Balberg, I.; Pinch, H. L. J. Magn. Magn. Mater. 1978, 7, 12-15.

41. Tafalla, D.; Salvador, P.; Benito, R. M. J. Electrochem. Soc. 1990, 137, 1810-1815.

42. Cushing, S. K. et al. J. Am. Chem. Soc. 2012, 134, 15033-15041.

43. Li, J. T. et al. 2013, 3, 47-51.

44. Khurgin, J. B.; Sun, G. Appl. Phys. Lett. 2009, 94, 221111.

45. Reilly, T. H.; Tenent, R. C.; Barnes, T. M.; Rowlen, K. L.; van de Lagematt, J. ACS Nano 2010, 4, 615-624. 


\section{Chapter 3: Hot Carrier Enhancement}

\subsection{Introduction}

As discussed in Chapter 2, for photovoltaics the primary goal of plasmonics is to increase light absorption in the semiconductor at energies above the band gap, allowing thinner films which decrease charge recombination and migration losses. The same goal exists for photocatalysis and photoelectrochemical cells, however the selection of semiconductors is made more difficult by the band gap restrictions necessitated by driving the redox reaction and photostability. Visible-light band gap semiconductors suffer from carrier recombination and migration issues as well as photostability problems. ${ }^{1-}$

${ }^{5}$ UV band gap metal oxide semiconductors absorb less than $5 \%$ of the solar spectrum, but their excellent photocatalytic properties still commonly lead to them outperforming visible light band gap alternatives. ${ }^{6-10}$

Therefore, in photocatalysis, plasmonic's goal is more often to increase the light absorption spectral range of wide-band gap semiconductors since the photostability issues of visible light band gap are intrinsic. This strategy is promising because not only do UV band gap semiconductors often already outperform their visible light counterparts, but Figure 1.1 in Chapter 1 showed that every additional $50 \mathrm{~nm}$ of light absorption in the 300-600 nm range further increases the efficiency by 1-3 times. Plasmonic's spectrally broad absorption cross section is therefore a promising route to achieve the $10 \%$ solar-to-hydrogen conversion needed for commercialization, ${ }^{11}$ but only if efficient energy transfer from the plasmon to semiconductor can be achieved.

Two plasmon-semiconductor energy transfer mechanisms are possible below and near the semiconductor band edge to address this goal. The first, hot carrier transfer, will be addressed in this Chapter. The second, plasmon-induced resonant energy transfer, will be addressed in Chapter 4.

\subsection{Theory Behind Hot Carrier Injection}

Recently great progress has been made in theoretically understanding how hot carriers are made by the plasmon and how these hot carriers may be transferred to a semiconductor. When the plasmon is first excited the electron oscillations are collective, leading to the large absorption and scattering moments. The collective electron oscillations break apart on a sub-10 fs time scale by electron-electron, surface, and other scattering processes. ${ }^{12-15}$ The scattering and dephasing leads to a non-collective distribution of hot electrons and holes, just as if the metal had absorbed light non-resonantly. The hot electrons and holes then thermalize by electron-phonon scattering with the lattice, losing their energy and returning to the ground state on a sub-100 ps time scale. ${ }^{12-15}$

The energetics of the hot carrier distribution is determined by the size and shape of the metal nanoparticle with a generation rate dependent on the non-radiative dephasing time. ${ }^{16-20}$ When the metal and semiconductor are in contact, the hot electrons (or holes) can overcome the interfacial Schottky barrier (Figure 3.1a). This process creates excited carriers in the semiconductor at energies where the plasmon absorbs, allowing the photoconversion range to be increased to energies that are sub-band gap. ${ }^{21-28}$ The promise of plasmonic hot carrier transfer is that $\sim 10^{21}$ carriers $/ \mathrm{cm}^{3}$, as determined by the free conduction electron density of the metal, are excited in the collective electron oscillations by a single photon. Therefore, a large amount of excited electrons or holes can be created to transfer to the semiconductor from a smaller volume with a higher probability than is possible by excitation of the semiconductor alone. If the hot carriers can be transferred to the semiconductor, the plasmon acts as a spectrally tuneable source of excited carriers. 

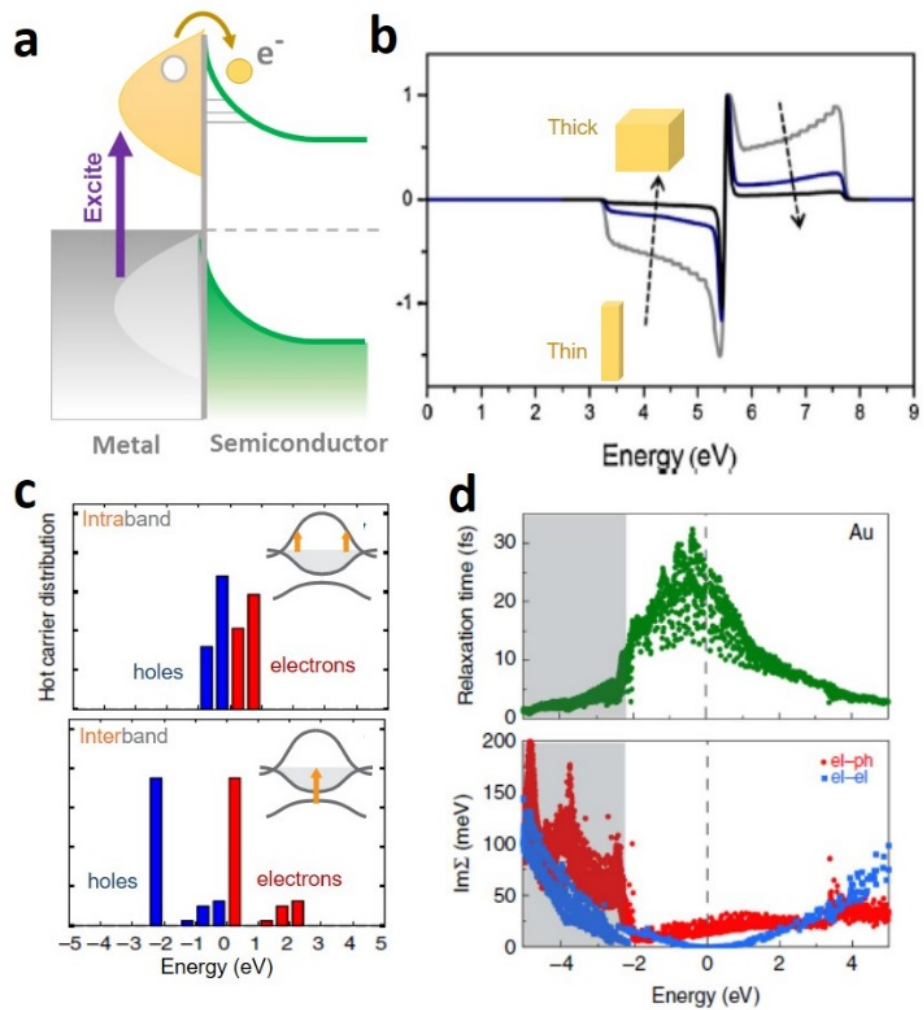

Figure 3.1. Plasmonic hot carrier generation and extraction. a, Hot carriers are generated with an energy above the Fermi level, which if sufficient to overcome the Schottky barrier, leads to excited carriers in the semiconductor when the plasmon absorbs light. b-c, For excitation of the metal (or plasmon) at energies above the interband threshold, the hot carrier distribution is asymmetric due to the involved transitions. For intraband excitation, the hot carrier distribution is symmetric, with small energy for large nanoparticles, but with energy equaling the plasmon resonance above the Fermi level $\left(E_{\mathrm{f}}\right)$ as the size is decreased. $\mathbf{d}$, As the hot carrier energy increases the lifetime and mean free path, as well as decay route, changes. b-d were reproduced with permission from References 16 and 19, Copyright (2013) American Chemical Society; Macmillan Publishers Ltd, copyright (2015).

The energy of hot carriers created by the plasmon is predicted to be depend on the size of the nanoparticle and the energy of the plasmon relative to the interband transitions of the metal. ${ }^{16-20}$ For resonances near the interband threshold, low energy hot electrons but high energy hot holes are produced (Figure 3.1b) because of the transition from a low-lying $d$ band to near the Fermi Level. For resonances at energies smaller than the interband threshold (Figure 3.1b), the produced carrier distributions are symmetric, with hot carrier energy above the Fermi level dependent on the degree to which momentum conservation is relaxed by size effects. Large metal nanoparticles and sheets, as well as SPP, produce hot electron hole distributions with $<1 \mathrm{eV}$ of energy above the Fermi level with most excitation energy going to momentum, especially in SPP modes. ${ }^{19,20}$ However as the size is decreased to a few nanometers and momentum conservation is relaxed by surface scattering (Figure 3.1c), the hot carrier distribution's energy increases above the Fermi level. ${ }^{16}$ A general rule is that for small metal nanoparticles, the hot carrier energy above the Fermi level is similar to the energy of the plasmon resonance in the optical absorption spectrum, and the plasmonic-hot carrier photocurrent follows the plasmon's absorption. ${ }^{16}$ For larger nanoparticles, the wavelength dependent transfer follows Fowlers theory $\left(h v-E_{\text {barrier }}\right)^{2}$, just as in a bulk metal. ${ }^{16}$

The Schottky barrier is necessary to separate the transferred hot electrons from the remaining holes in the metal or vice versa, ${ }^{27,29}$ and if not present, the hot carriers will quickly recombine. The spatial separation 
of excited carriers must equally be taken into account to prevent charge equilibration from stopping further electron or hole transfer. This is made difficult by the differing lifetime of hot carriers with energy, Figure 3.1d. Near the Fermi level electron-electron scattering limits lifetimes and decay lengths to 30-50 nm, but for interband transitions electron-phonon scattering dominates, decreasing the mean free path to a few nanometers. ${ }^{19}$ Electron-phonon scattering also thermalizes the hot carrier distribution to lower energies quicker, limiting the ability to transfer over a barrier, whereas electron-electron scattering mainly contributes to dephasing rates. The increase in electron-phonon scattering is a source of increased damping at the interband transition, and deserves more study to see if interband versus intraband hot carriers can be extracted more efficiently.

Based on these conditions, efficient hot carrier transfer to enhance photocatalysis is a difficult balance of maintaining high hot carrier energies to overcome the interfacial barrier while extracting both charge carriers on femtosecond to picosecond time scales. Despite the plasmon's large absorption cross section, these conditions place current theoretical enhancements at below $1 \% .{ }^{48}$ To date, most hot carrier-based devices have fallen within this theoretical upper limit. However, it is difficult to separate the effects of hot carrier transfer versus enhancement by the near field in these studies. Further, the theoretical predictions of hot carrier creation depending on plasmon energy and geometry have yet to be investigated, and neither has the charge transfer and equilibration time scales. This is further complicated by the ability of gold nanoparticles to store transferred charge from a semiconductor or act as Ohmic transport layers within the device. These issues are addressed in the following section.

\subsection{Gold Nanoparticles: Electron Relay versus Hot Electron Source}

Solar-to-chemical energy conversion efficiency is determined by the balance of light absorption, charge separation, charge migration and charge recombination in photocatalysts, with an ideal semiconductor photocatalyst excelling in all four categories. ${ }^{30,31}$ However, it has proven to be impossible to optimize a single semiconductor material for all these processes so far. Titanium oxide $\left(\mathrm{TiO}_{2}\right)$ remains the most commonly used wide-band gap semiconductor photoanode in photoelectrochemical cells (PECs) due to its high catalytic activity, good stability, long minority diffusion length and low cost. However, it absorbs only ultraviolent light because the band gap is $3.2 \mathrm{eV}$ for anatase $\mathrm{TiO}_{2}$, limiting overall efficiency. ${ }^{32,33}$ Doping with either transition metal or non-metal ions can extend its light absorption into the visible light region, but doping introduces trap states and charge carrier recombination centers, which limits the visible-light photocatalytic activity. ${ }^{33-37}$

Alternatively, wide-band gap metal-oxide semiconductors can be combined with a narrow-band gap semiconductor to form a heterostructure. This allows the excellent charge transport and recombination properties of the wide-band gap semiconductor to be taken advantage of while simultaneously extending the light absorption range. For example, semiconductor quantum dots (QDs) have been commonly used as photosensitizers due to their high absorption cross-section and controllable absorption spectrum through nanoparticle radius. ${ }^{38-40}$ Energetically favorable band alignment is necessary between the QD and the metal oxide in order to promote efficient interfacial charge transfer and chemical stability. Ideally, upon illumination, electrons excited in the QD transfer into the conduction band (CB) of the metal oxide while the holes transfer to the photoelectrode/electrolyte interface, extending the spectral range of the absorption without sacrificing recombination lifetimes. ${ }^{41}$ In reality, slow electron injection and high charge recombination rates at the interfaces often remains problematic. ${ }^{42-47}$ In organic dye-sensitized metal oxide electrodes, the photo-generated electrons quickly transfer into metal oxide. However, the large density of 
surface trap states in QD-metal-oxide heterostructures and the subsequent charge accumulation at the surface slows down the transfer of excited electrons and holes, increasing the charge recombination rate and consuming the photogenerated charge carriers. ${ }^{48}$ It is essential to promote efficient interfacial charge transfer from the QDs to the metal oxide to enable high efficiency energy conversion in QD-sensitized solar energy devices.

As discussed previously in this chapter, another option for extending the light absorption of wide-band gap semiconductors is to use plasmonic metal nanostructures as the photosensitizers instead of QDs. In Chapter 1, it was shown that localized surface plasmon resonance (LSPR) corresponds to the collective oscillation of surface electrons, with an oscillation frequency that is dependent on size, shape, and metal of the nanostructure. The energy stored in the plasmon can be transferred to a semiconductor by hot carrier transfer (also referred to as direct electron transfer (DET) as discussed in Section 3.2) or plasmon induced resonant energy transfer (PIRET) as will be discussed in Chapter 4. ${ }^{49-53}$ DET occurs through the transfer of plasmonic hot electrons over the interfacial Schottky barrier, whereas PIRET occurs through non-radiative dipole-dipole coupling between the plasmon in the metal and electron-hole pairs in the semiconductor. As will be discussed further in Chapter 4, unlike organic dyes and QDs which require energetically favorable band alignment for transfer, the energy transfer through PIRET is dependent on spectral overlap in the absorption spectrum.

Photocurrent enhancement has already been observed in ternary heterostructures comprised of QDs, Au nanoparticles and metal-oxide semiconductor nanoparticles. ${ }^{54-58}$ The plasmonic photosensitization effect has yet to be reported for this structure and the mechanism of photoconversion enhancement by the $\mathrm{Au}$ nanoparticles in the ternary structures remains poorly understood. Additionally, while the charge-transfer rate in QD-metal-oxide heterostructures has been frequently measured, the resulting transfer mechanism is largely inferred from the average lifetime change in a multi-exponential fit to the QD exciton bleach. ${ }^{59-66}$ The uncertainty of multiple exponential fitting parameters has led to divergent reports of the electron transfer time from femtoseconds to nanoseconds, although the relative change in lifetime with energybarrier height is impressively consistent with predictions from Marcus theory. ${ }^{59-66}$ Nevertheless, the inherently non-exponential nature of Auger decay and other recombination/transfer mechanisms in the semiconductor further blurs the charge transfer mechanism when the measured rates are interpreted from the metal-oxide semiconductor's decay instead of the QD bleach. The acceptor dynamics are commonly found not to obey the first-order rate equation adapted from charge transfer models suitable for dyes, as recently evidenced by the discoveries of Auger-based electron transfer and diffusion-based Auger recombination in the inverted Marcus region. ${ }^{67,68}$ The difficulty in interpreting transient-absorption measurements has led to ambiguity in how the charge transfer precedes once carriers have gone from the QD into the interfacial surface states, despite this being the critical step to charge separation. This ambiguity in interpretation can be overcome by inverting the transient-absorption data to provide rates and lifetime versus carrier density.

In the following Section, ${ }^{27}$ a sandwich-structured $\mathrm{CdS}-\mathrm{Au}-\mathrm{TiO}_{2}$ nanorod array, which is vertically aligned on a fluorine-doped tin oxide (FTO) substrate, acts as the photoanode in a PEC for solar hydrogen generation. The gold nanoparticles sandwiched between the $\mathrm{TiO}_{2}$ nanorod and the CdS QD are shown to play a dual role in enhancing the solar-to-hydrogen conversion efficiency. First, the Au nanoparticles function as an electron relay that facilitates charge transfer between the CdS QDs and $\mathrm{TiO}_{2}$. Secondly, the Au nanoparticles act as a plasmonic photosensitizer that extends the photoconversion of the photoanode to $725 \mathrm{~nm}$. The role of the Au nanoparticles is discerned using a newly adapted analysis method, which disentangles the multiple contributions to the transient-absorption signals by directly mapping out the 
coupled rate equation which governs charge transfer. This procedure reveals the key role of the interfacial trap states in QD-metal-oxide heterostructures, in which the charge transfer can proceed through trap-state Auger scattering in the interface states with back-transfer or increased charge separation occurring on the long-timescale.

When the Au nanoparticles are included in the heterostructure, the trap-based Auger scattering rate is reduced, the transfer rate increases into the $\mathrm{CB}$ of $\mathrm{TiO}_{2}$, and the back-transfer is reduced independent of the excitation wavelength. The inverted transient-absorption analysis reveals not only the dynamics of Au as a transfer channel, but also its ability to overcome some of the deficiencies related with interfacial trap states in the $\mathrm{CdS}-\mathrm{TiO}_{2}$ heterostructures. Additionally, the plasmonic electron transfer mechanism is identified as DET into the $\mathrm{TiO}_{2}$, with the transfer of hot electrons depending on the spectral position of the plasmon and the back transfer dynamics depending on the hot electron distribution energy relative to the Schottky barrier, therefore addressing the questions raised in Section 3.2

\subsubsection{Methods}

Synthesis of $\mathrm{TiO}_{2}$ nanorod array on FTO: The $\mathrm{TiO}_{2}$ nanorod array was fabricated on the FTO substrate with a hydrothermal method. ${ }^{69,70}$ Briefly, $0.8 \mathrm{~g}$ of titanium butoxide was dissolved into $60 \mathrm{~mL}$ of $6 \mathrm{M} \mathrm{HCl}$ aqueous solution, and then transferred into a Teflon-lined steel autoclave with a capacity of $120 \mathrm{~mL}$. The FTO substrates were placed against the Teflon wall with the FTO side facing down. The autoclave was kept in an oven at $150{ }^{\circ} \mathrm{C}$ for $24 \mathrm{~h}$, and then cooled down to room temperature. $\mathrm{The}^{\mathrm{TiO}} \mathrm{O}_{2}$ nanorods were cleaned with deionized (DI) water and ethanol.

Decoration of Au nanoparticles on the $\mathrm{TiO}_{2}$ nanorods: $10 \mathrm{mM} \mathrm{HAuCl}_{4}$ aqueous solution was tailored to $\mathrm{pH} 4.5$ with the $\mathrm{NaOH}$ solution. The $\mathrm{TiO}_{2}$ nanorod array was immersed into the $\mathrm{HAuCl}_{4}$ aqueous solution for $4 \mathrm{~h}^{.1}{ }^{11}$ The $\mathrm{TiO}_{2}$ nanorod array was then washed with DI water and annealed at $450{ }^{\circ} \mathrm{C}$ for $2 \mathrm{~h}$. This led to Au nanoparticles on the surface of $\mathrm{TiO}_{2}$ nanorod.

Fabrication of $\mathrm{CdS}-\mathrm{Au}-\mathrm{TiO}_{2}$ sandwich structure: $\mathrm{CdS}$ QDs were deposited on the surface of the Audecorated $\mathrm{TiO}_{2}$ nanorod array with a chemical bath deposition. ${ }^{70} 1 \mathrm{mM} \mathrm{CdSO}_{4}$ and $5 \mathrm{mM}$ thiourea were dissolved into $1 \mathrm{M}$ ammonia solution. The $\mathrm{Au}-\mathrm{TiO}_{2}$ nanorod array was then immersed into the CdS precursor solution, and heated in a water bath at $60{ }^{\circ} \mathrm{C}$ for $10 \mathrm{~min}$. After deposition of CdS, the nanorod array was washed with DI water and annealed in a $\mathrm{N}_{2}$ flow at $400{ }^{\circ} \mathrm{C}$ for $2 \mathrm{~h}$.

Characterization: The morphology and structure of the nanorod array were observed with a fieldemission scanning electron microscope (FE-SEM) (JEOL 7600F) and a transmission electron microscope (TEM, JEOL JEM 2100F). The light-absorption properties were characterized by diffuse-reflection with a Shimadzu 2550 UV-Visible spectrometer equipped with an integrating sphere (UV 2401/2, Shimadzu). The chemical status of elements was analyzed with X-ray photoelectron spectroscopy (XPS, PHI 5000 Versa Probe system, Physical Electronics).

Photoelectrochemical performance measurement (PEC): The PEC performance was measured using a Gamry Reference 3000 ${ }^{\mathrm{TM}}$ Potentiostat/Galvanostat/ZRA Instrument. A $300 \mathrm{~W}$ Xe lamp with an AM 1.5G filter was used as the light source. An aqueous solution containing $0.25 \mathrm{M} \mathrm{Na}_{2} \mathrm{~S}$ and $0.35 \mathrm{M} \mathrm{Na}_{2} \mathrm{SO}_{3}(\mathrm{pH}$ 12) was used as the electrolyte and bubbled with $\mathrm{N}_{2}$ for 30 min prior to measurement. A Pt wire was employed as the counter electrode and $\mathrm{Ag} \mid \mathrm{AgCl}$ as the reference electrode. Band-pass and long-pass filters were used to adjust the wavelength region of incident light.

Transient-Absorption measurements: The $100 \mathrm{fs}, 800 \mathrm{~nm}$ pulses of a $1 \mathrm{kHz}$ Ti:Sapphire amplifier were coupled into an optical parametric oscillator (OPA), then either frequency doubled in $\beta$-barium borate (BBO) to create a $400 \mathrm{~nm}$ pump or mixed with the OPA output to create a $480 \mathrm{~nm}$ pump by sum frequency 
generation. A pump power of $2 \mathrm{~mW}$ was used in both cases with a radius of $\sim 200 \mu \mathrm{m}$. A white-light supercontinuum was created by tightly focusing the $800 \mathrm{~nm}$ output in a sapphire plate, using circular polarization to increase the stability. The white-light probe has a spot size of $<100 \mu \mathrm{m}$ and spans $450-$ $1000 \mathrm{~nm}$, but wavelengths around $800 \mathrm{~nm}$ are excluded from the spectra because the continuum generated is strongly peaked at this wavelength beyond the dynamic range of the spectrometer, making acquisition unreliable in this region. White-light transient-absorption spectroscopy was performed on the same samples used in the PEC testing. The data is converted by the inversion analysis (explained in the next section) to create plots of the time derivative of carrier density verses carrier density. The log-log plot transforms $\frac{d N}{d t}=$ $-N^{x} / \tau$ into $\log \left(\frac{d N}{d t}\right)=-x \cdot \log (N)-\log (1 / \tau)$, meaning that the relative position of the plotted data and the intercept determines the relaxation rate while the slope gives the nonlinear order of the relaxation dynamics.

Motivation, Explanation, and Implementation of Pump-Probe Inversion Analysis It is difficult to interpret the effect of heterostructuring on the acceptor and donor lifetime, even if only the transfer out of the QD donor and not the complex dynamics of the trap states are considered. This can be seen in Figure 3.2, where a representative signal is shown for the $\mathrm{CdS}-\mathrm{TiO}_{2}$ samples pumped at $400 \mathrm{~nm}$ (exciting $\mathrm{TiO}_{2}$ and $\mathrm{CdS}$ ) and $480 \mathrm{~nm}$ (exciting CdS only) while being probed at the $\mathrm{TiO}_{2}$ electron-trap state absorption. The decay dynamics of the $\mathrm{TiO}_{2}$ and $\mathrm{CdS}$ alone pumped at $400 \mathrm{~nm}$ are shown for comparison. It is seen the initial transfer dynamics seem quicker in the heterostructure similar to CdS alone, while the long timescale transfer dynamics depend on whether carriers are excited in CdS alone or CdS and $\mathrm{TiO}_{2}$.

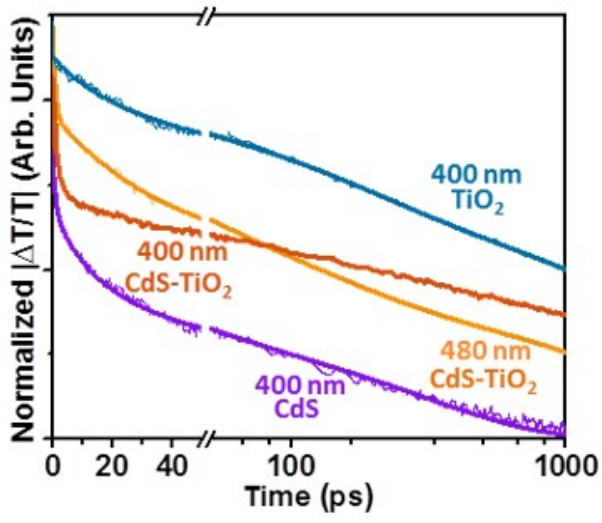

Figure 3.2. Transient-absorption signals for $\mathrm{CdS}, \mathrm{TiO}_{2}$ and $\mathrm{CdS}-\mathrm{TiO}_{2}$ at $400 \mathrm{~nm}$ and $480 \mathrm{~nm}$ pump wavelengths. The curves are shifted for comparison.

Traditionally, each decay curve is fit with 1 to 5 exponential terms and the relative lifetimes compared, giving a quantitative interpretation to the information gained by visual inspection. However, charge transfer lifetimes inferred from this fitting vary greatly depending on the number of exponentials used in the fit, the time scales measured, and how the average change in lifetime is defined. ${ }^{59-66}$ Comparison of lifetimes is further encumbered when contributions from both the donor and acceptor exist at the measured probe wavelength, as evidenced in Figure 3.2. It is therefore difficult to conclude mechanistic details from the exponential fit, unless systematic sample design is used to vary a structural parameter against which the change in lifetimes can be compared. Even in this case only the trend and not the absolute lifetimes are guaranteed quantitative. 

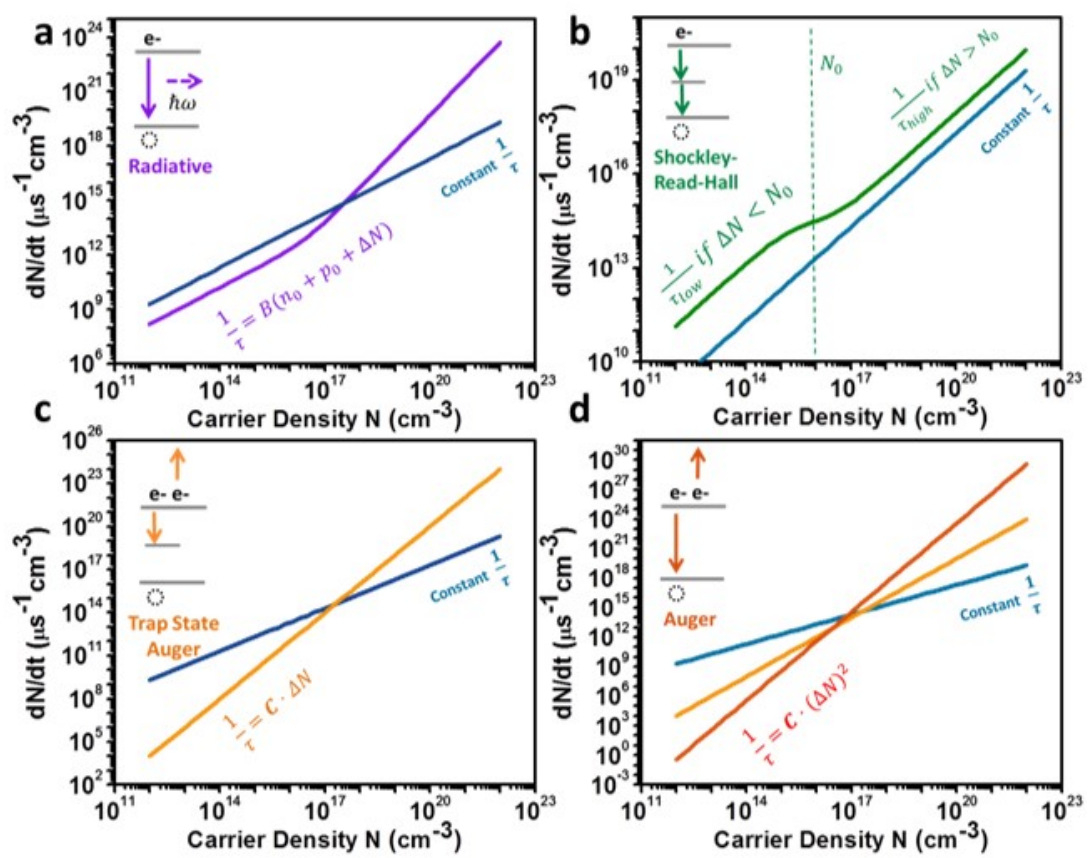

Figure 3.3. Nonlinear nature of relaxation mechanisms in semiconductors. A schematic diagram, mathematical form, and a constant lifetime curve (exponential decay) is shown in each graph for comparison. a, At low carrier injection levels radiative relaxation which occurs by the emission of a photon is first order, or linear on the slope of rate verses carrier density. At higher injection levels the radiative relaxation becomes second order. b, Shockley-Read-Hall recombination occurs through mid-gap trap states. SRH transitions between two first order relaxation rates depending on the excited carrier density relative to the doping level. The transition between these two areas depends on the position of the trap states in the band gap, and several other parameters as shown in more detail in References 71-73. c, Trap-state based Auger scattering is a second order process that occurs by an electron relaxing to a trap state by giving its energy to a second electron. The rate has a slope of 2 on a log-log plot. d, Auger recombination is a third order process where an electron and hole recombine by giving energy to a third electron, leading to a slope of three on a log-log plot. In all cases the intercept of the log-log plot gives the rate coefficient of the mechanism. The given curves are based off parameters given in Ref. 73.

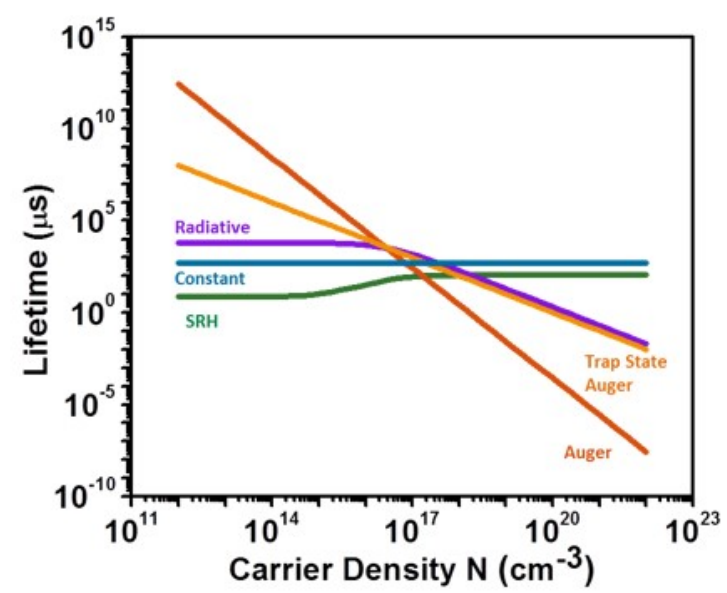

Figure 3.4. Instantaneous lifetime for each of the recombination mechanisms shown in Figure 3.3. The curves are obtained by dividing the carrier density by the derivative of the carrier density verses time, inverting the rate equation. The given curves are based off parameters given in Ref. 71-73, showing how nonlinear relaxation terms dominate during high carrier injection, which correspond to short time scales in transient absorption. On long time scales the carrier dynamics become constant. 
The reason multiple exponential fits fail to accurately reflect dynamics is that the decay kinetics of a semiconductor are non-exponential by nature, with the dominant mechanisms such as Shockley-Read-Hall, radiative, surface recombination, and Auger scattering all depending explicitly on carrier density except in cases of low level injection. The different recombination mechanisms are illustrated in Figure 3.3 and Figure 3.4, along with their dependence on excited carrier density. The nonlinear dependence on the excited carrier density forbids simple exponential solutions of the rate equation. Although analytical solutions exist for nonlinear kinetics, the mechanism must be known before hand to select the appropriate model, as several possible models will often accurately describe the decays and be fit equally. Further, the common multiple exponential solution assumed for fitting cannot be obtained for a semiconductor treated as a two-level system even if the relaxation rate only depends linearly on carrier density. This is because additional linear rates just add inversely to the overall lifetime

$$
\begin{aligned}
& \frac{\partial \Delta N}{\partial t}=-\frac{\Delta N}{\tau} \\
& \text { with } \\
& \frac{1}{\tau}=\frac{1}{\tau_{\text {rad }}}+\frac{1}{\tau_{S R H}}+\frac{1}{\tau_{\text {auger }}}+\cdots
\end{aligned}
$$

assuming all lifetimes are independent of injected carrier density. This is only the case in low injection, with the actual governing rate equation resembling

$$
\frac{\partial \Delta N}{\partial t}=-\frac{\Delta N}{\tau_{S R H}(\Delta N)}-\frac{\Delta N^{2}}{\tau_{R A D}}-\frac{\Delta N^{3}}{\tau_{A U G E R}}-\cdots
$$

which no longer has a simple exponential solution.

Despite the indeterminate nature of multiple exponential fitting and that a single exponential fit only holds in low-level injection, using multiple exponential fits is prevalent because it is otherwise impossible to explain the relaxation data. The necessity of a multiple exponential fit for a single semiconductor can be seen for $\mathrm{TiO}_{2}$ in Figure 3.2, which in-spite of being dominated by SRH recombination with little Auger or radiative effects, still has a non-linear (or non-exponential) slope on a log plot. The non-exponential dynamics are even more pronounced in the CdS QD's, Figure 3.2. This is because the small volume of the QD means a single excited electron-hole pair translates into a charge density of $\sim 10^{22}$ to $10^{23}$ electrons $/ \mathrm{cm}^{3}$, which leads to Auger scattering dominating the initial recombination dynamics. The decay mechanism then evolve to radiative or SRH recombination as the charge carrier density decreases at longer timescales.

The non-exponential dynamics are further magnified when the CdS and $\mathrm{TiO}_{2}$ are combined in the heterostructure, Figure 3.2. Even if the two semiconductors had single exponential dynamics alone, the solution of the coupled first order rate equations is no longer single exponential, Figure 3.5a, as the charge transfer from the donor adds an exponential dependence to the rate equation of the acceptor. In a first order transfer model, the transfer reduces the lifetime of the donor by a constant rate but leads to non-single exponential dynamics for the acceptor. This allows the difference in average lifetime in the donor before and after coupling to be easily used to calculate the transfer rate, but disrupts knowledge of transfer dynamics once the carriers leave the donor. This explains the success of probing only the QD lifetime to determine the transfer time, but the difficulty in relating the determined transfer rate to the resulting acceptor dynamics. The first order coupled rate equation model predicts a rise in the acceptors decay as the donor relaxes. This trend can be found in organic dye-metal oxide or organic dye-organic dye charge transfer systems $^{74,75}$ but is generally absent in QD-metal oxide systems and organic dye-metal oxides with defects. ${ }^{76,77}$ Therefore, while the transport out of the QD donor can be approximated by the change in average lifetime and even directly predicted by Marcus theory, the subsequent dynamics in the metal oxide acceptor cannot, leaving conflicting results about how charge transfer continues after the initial stage. 
To overcome these issues, we have adapted an inversion analysis technique first proposed by Linnros ${ }^{71-}$ ${ }^{73}$ for single semiconductors using free carrier absorption (FCA). The multiple exponential fit has remained in wide use despite its limitations because it is otherwise difficult to fit non-exponential dynamics: Not only must the coupled rate equations be solved, but the appropriate recombination mechanisms must be guessed and the unknown parameters found, leaving the original problem even more undetermined. However, instead of solving the rate equation the transient-absorption data can be transformed by taking the time derivative. This alters the transient-absorption data from the solution of the underlying rate equation back to the rate equation itself.
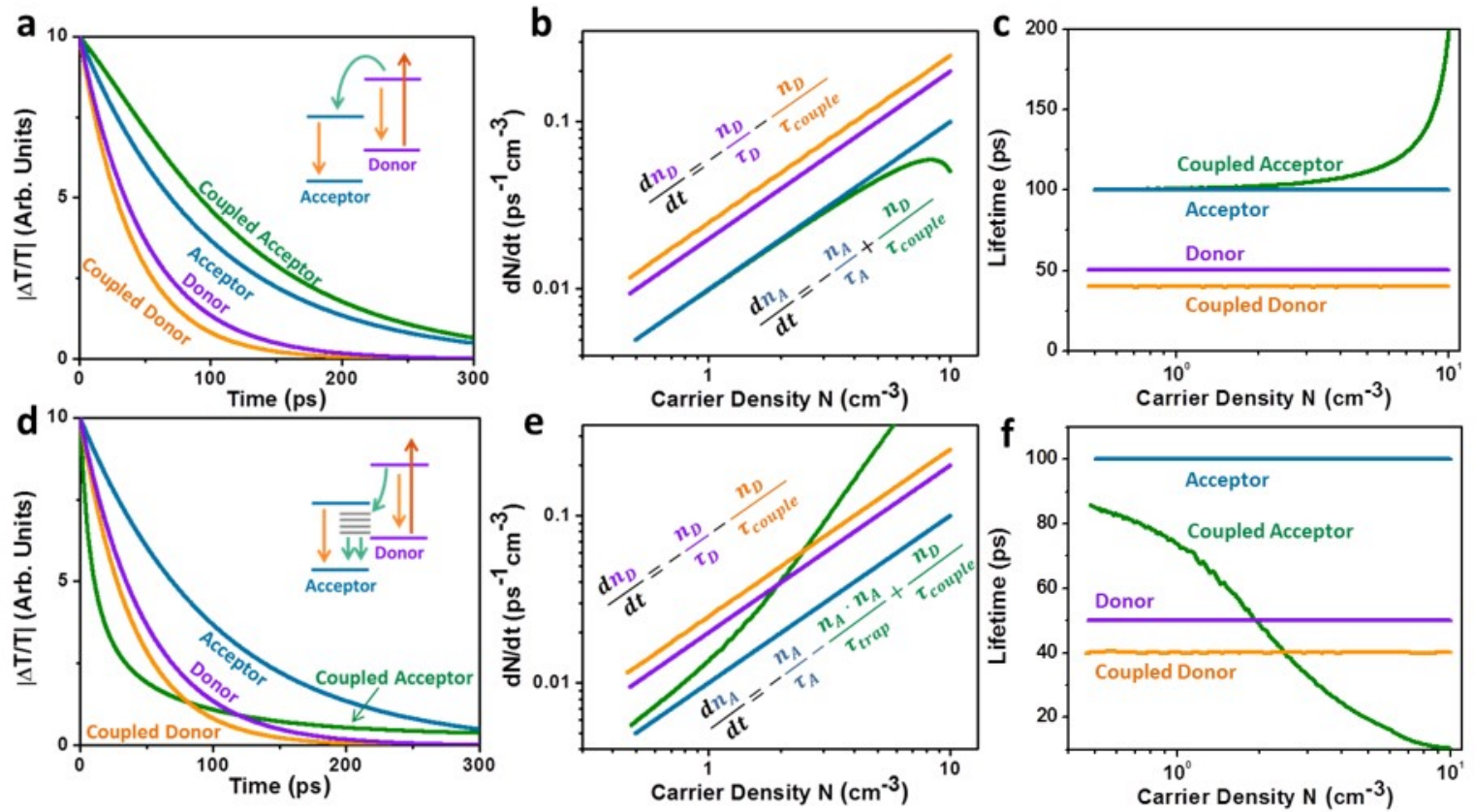

Figure 3.5. Modeled relaxation dynamics in heterostructures. The decay is shown for a, a simple first order coupled rate equation and $\mathbf{d}$, a coupled rate equation with trap-state Auger recombination in the acceptor. By taking the derivative of the respective decay dynamics, $\mathbf{b}$ and $\mathbf{e}$, the form of the underlying rate equation is seen. In both cases the donor has an initially higher rate due to charge transfer. The charge transfer into the acceptor leads to a lower initial rate, $\mathbf{b}$, for the first order coupled rate equation, but the addition of trap-state Auger scattering creates an initially higher relaxation rate. After charge transfer is complete the lifetime returns to that of the acceptor, as seen by the instantaneous lifetime in $\mathbf{c}$ and $\mathbf{f}$ found by inverting the rate equation of $\mathbf{b}$ and $\mathbf{e}$.

The form of the underlying rate equation can therefore be easily found experimentally by plotting the derivative of the transient absorption data verses the excited carrier density, as shown in Figure 3.5b for the coupled first order example. On a log-log plot, an exponential decay becomes a straight line with a shift above or below corresponding to a shorter or longer lifetime, respectively. Any curvature away from a straight line of slope one corresponds to non-exponential dynamics, with the order given by the slope. This gives the different recombination mechanisms unique forms, as shown in Figure 3.3, removing the ambiguity found in the decay data. Further, inverting the rate equation by dividing the derivative by the carrier density $\frac{d \Delta N}{d t} / \Delta N$ directly gives the instantaneous lifetime, allowing the relaxation constants to be determined without a fitting procedure. This can be seen by grouping the nonlinear dependence on carrier density into a carrier density dependent lifetime 


$$
-\frac{\partial \Delta N}{\partial t}=\Delta N\left(\frac{1}{\tau_{S R H}(\Delta N)}+\frac{1}{\tau_{R A D}(\Delta N)}+\frac{1}{\tau_{\text {auger }}(\Delta N)}\right)=\frac{\Delta N}{\tau(\Delta N)}
$$

so that

$$
\frac{1}{\tau(\Delta N)}=-\frac{\frac{\partial \Delta N}{\partial t}}{\Delta N}
$$

As shown in Figure 3.4, the instantaneous lifetime verses carrier density also helps uniquely show the recombination mechanism, with a traditional exponential solution again being a constant verses excited carrier density

The utility of this technique in analyzing heterostructure is shown in Figure 3.5. If both donor and acceptor are measured, the inversion of the data transforms the pump probe signal into direct measurement of the coupled rate equation, regardless of the presence of non-exponential relaxation dynamics. The acceptor data in Figure 3.5a requires a multiple exponential fit despite the acceptor and donor having single exponential kinetics, with the fitted lifetimes of 40 ps and 115 ps differing from the true acceptor lifetime of 100 ps or the coupling time of 200 ps. The inverted data in Figure 3.5b and 3.5c is seen to be much easier to interpret. After coupling, the donor lifetime of $50 \mathrm{ps}$ is seen to be reduced by the coupling constant of 200 ps to 40 ps, directly mapping the donor's side of the rate equation (inset in Figure 3.5b). The acceptor rate is seen to be initially slower, corresponding to the exponential addition of carriers from the donor population as it decays. After the donor has decayed the lifetime of the acceptor returns to its own dynamics, illuminating the origin of the apparently multiple exponential dynamics in Figure 3.5a.

Procedure for Applying Inversion Analysis The difficulty in experimentally applying the inversion analysis is smoothly taking the derivative of noisy data. Although several algorithms exist for smoothing the derivative, the results were found to tend to distort the curvature of the decay. Instead of smoothing the derivative, this problem was solved by utilizing the well-known ability of an over-parameterized multiexponential decay to fit the transient absorption data and act as a filter. The inversion analysis therefore becomes as simple as performing the standard multi-exponential analysis, then taking the derivative of the result and inverting the absorption. As long as the data is completely fit, the derivative destroys the multiexponential assumption and gives the underlying rate equation. The inversion is achieved by first transforming the transient absorption into a carrier density ${ }^{71-73}$

$$
\frac{\Delta A}{A}=\frac{I_{0} \exp \left(-\left(\alpha_{\text {probe }}+\sigma \Delta N\right) d\right)-I_{0} \exp \left(-\alpha_{\text {probe }} d\right)}{I_{0} \exp \left(-\alpha_{\text {probe }} d\right)}=\exp (-\sigma \Delta N d)-1
$$

so that

$$
\Delta N=-\frac{1}{\sigma d} \ln \left(1 \pm\left(\frac{\Delta A}{A}\right)\right)
$$

where the \pm is used to ensure the excited carrier density is positive whether the signal is a bleach or an excited state absorption. The absorption cross section $\sigma$ acts as a calibration between the number of excited carriers in the sample, found by

$$
N_{\text {exc }}=\frac{I_{0}}{\hbar \omega} \cdot \frac{\alpha_{\text {pump }}(1-R)^{2} \exp \left(-\alpha_{\text {pump }} d\right)}{1-R^{2} \exp \left(-\alpha_{\text {pump }} d\right)} \approx \frac{I_{0} \cdot \alpha_{\text {pump }}}{\hbar \omega}
$$

where $N_{\text {exc }}=\Delta N$ and the measured increase in transient absorption signal is

$$
\frac{\Delta A}{A}=\exp (-\sigma \Delta N d)-1 \approx \pm \sigma N_{\text {exc }} d \text {. }
$$

The cross section can therefore be found by the slope of the maximum of the transient absorption verses several excitation intensities. In these equations, \pm depends on if the signal is an excited state absorption or bleach, $I_{0}$ is the incident flux in $\mathrm{J} / \mathrm{cm}^{2}, \alpha$ is the absorption coefficient in $\mathrm{cm}^{-1}, \hbar \omega$ is the energy of the pump, $R$ is the reflection coefficient, $d$ is the sample thickness in $\mathrm{cm}$, and $N_{\text {exc }}$ is the number of excited 
carriers per $\mathrm{cm}^{3}$. After this is complete, the data can be fit with an arbitrary number of exponentials so the decay is completely represented, $i$. e . the exponentials are simply acting as a filter of the noise. The derivative of the data can then be taken, comparing to the unfiltered (or unfit) derivative to ensure accuracy.

In the data reported, the plots were shifted slightly for comparison by multiplying the derivative of the carrier density and carrier density by a constant. This is equivalent to changing the effective absorption cross section and does not change the dynamics, especially in the case of a constant lifetime like that measured for the donors. The fitting coefficients given in the following sections were calculated without shifting the data for comparison. The small shifts are purely to aide in visualizing the difference in high and low carrier dynamics between the different pump wavelengths and heterostructures.

\subsubsection{Results}

Microstructure Single-crystalline rutile $\mathrm{TiO}_{2}$ nanorod arrays were grown on a FTO substrate with a hydrothermal process (Figure 3.6). ${ }^{69,70}$ The $\mathrm{TiO}_{2}$ nanorods were $\sim 2.5 \mu \mathrm{m}$ high and 150-200 $\mathrm{nm}$ in a diameter (Figure 3.6b and 3.6c). Au nanoparticles with an average size of $11.5 \pm 3 \mathrm{~nm}$ were deposited on the $\mathrm{TiO}_{2}$ nanorod with a photo-reduction method ${ }^{78}$ and a uniform $15 \mathrm{~nm}$ thick CdS QD layer was subsequently deposited with a chemical bath deposition method $^{70}$ to form a sandwich nanorod structure, as shown in Figure 3.6. This structure was also confirmed by the TEM and HRTEM images as well as the XPS analysis in depth in Reference 27.

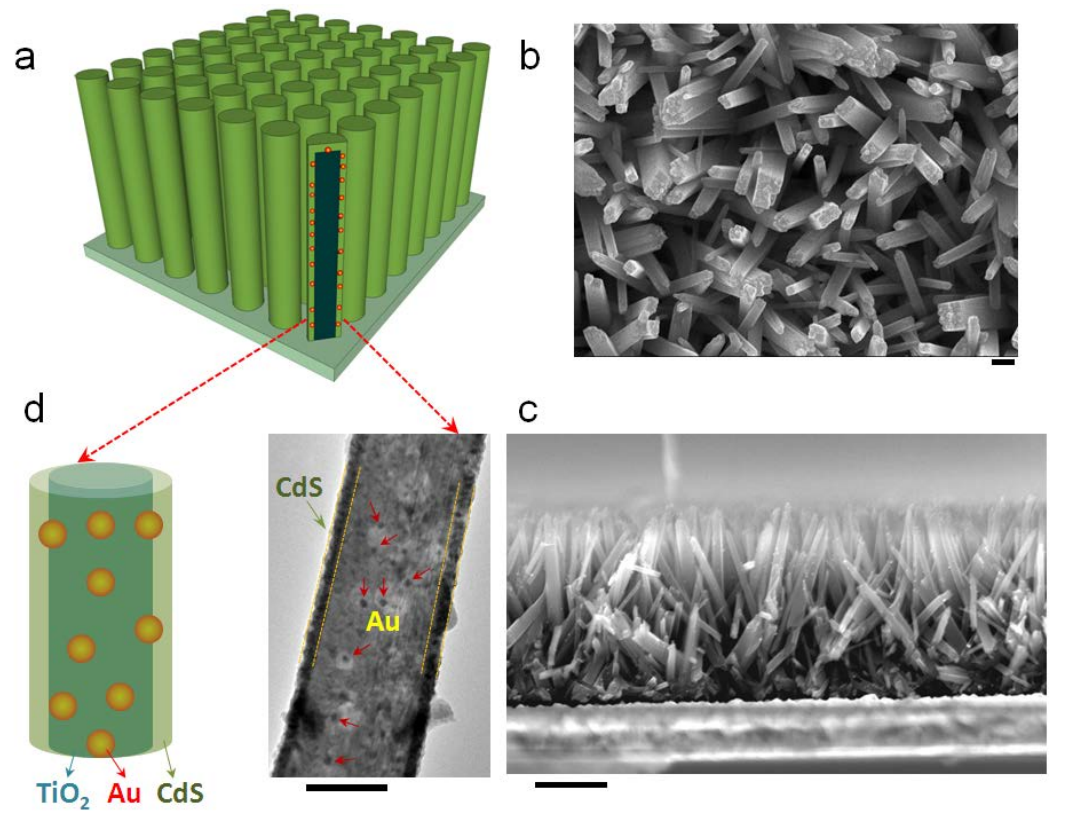

Figure 3.6. Microstructure of the $\mathrm{CdS}-\mathrm{Au}-\mathrm{TiO}_{2}$ sandwich nanorod array. a, Scheme for the sandwich nanorod array on the FTO substrate; $\mathbf{b}$, Top-view, and c, cross-section view of CdS-Au-TiO ${ }_{2}$ nanorod array; d, TEM image of a single sandwich nanorod. Scale bars: (b) $200 \mathrm{~nm}$, (c) $1 \mu \mathrm{m}$, and (d) $100 \mathrm{~nm}$.

Optical Absorption Figure 3.7 shows the UV-Visible absorption spectra obtained from the $\mathrm{TiO}_{2}$ nanorod array and the $\mathrm{CdS}-\mathrm{TiO}_{2}$ arrays in the absence and presence of Au nanoparticles. The as-prepared $\mathrm{TiO}_{2}$ nanorod array displayed a sharp absorption edge at around $410 \mathrm{~nm}$, which matched the band gap of rutile titanium oxide. At longer wavelength, below the energy of the band gap of both $\mathrm{TiO}_{2}$ and $\mathrm{CdS}-\mathrm{TiO}_{2}$, the absorption had a background from the FTO. Sensitization of $\mathrm{TiO}_{2}$ with the CdS QDs extended the absorption range up to $525 \mathrm{~nm}(2.36 \mathrm{eV})$. The presence of Au nanoparticles embedded between $\mathrm{TiO}_{2}$ and 
CdS further increased the absorption at the wavelengths shorter than the band edge of CdS and extends the absorption range up to $725 \mathrm{~nm}$. The strong absorption band centered at $610 \mathrm{~nm}$ corresponded to the LSPR of the Au nanoparticles. The LSPR peak was sensitive to the size, shape and the surrounding environment. For 10-nm spherical Au nanoparticles in an aqueous solution the LSPR peak is typically centered at 520 $\mathrm{nm} .^{51,78}$ The LSPR peak red-shifted to $550 \mathrm{~nm}$ when the Au nanoparticles were deposited on the surface of $\mathrm{TiO}_{2}$. The LSPR peak of the Au nanoparticles sandwiched between the $\mathrm{TiO}_{2}$ nanorod and the CdS QD layer further red-shifted to $610 \mathrm{~nm}$ due to the large refractive index of the CdS layer.

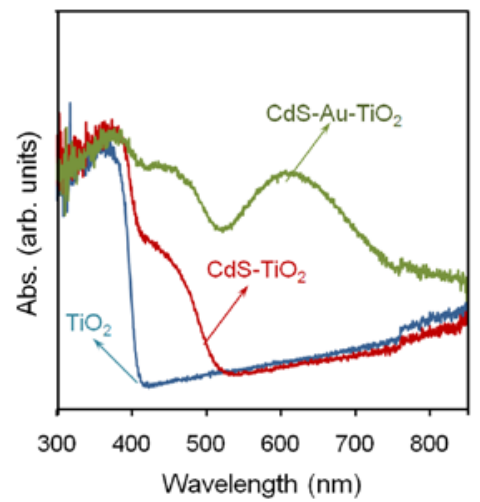

Figure 3.7. UV-Visible absorption spectra obtained from the $\mathrm{CdS}-\mathrm{TiO}_{2}$ nanorod arrays with and without $\mathrm{Au}$ nanoparticles.

Photoelectrochemical Performance Photoelectrochemical performance of the nanorod array photoanode was measured with a three-electrode cell with a Pt wire as the counter electrode and $\mathrm{Ag} \mid \mathrm{AgCl}$ as the reference electrode. An aqueous solution containing $0.35 \mathrm{M} \mathrm{Na}_{2} \mathrm{SO}_{3}$ and $0.25 \mathrm{M} \mathrm{NaS}_{2}$ was used as the electrolyte. The CdS- $\mathrm{TiO}_{2}$ nanorod array photoanode exhibited a photocurrent of $3.10 \mathrm{~mA} / \mathrm{cm}^{2}$ at $0 \mathrm{~V}$ (vs. $\mathrm{Ag} \mid \mathrm{AgCl})$ under a full solar spectrum irradiation with simulated solar light at $100 \mathrm{~mW} / \mathrm{cm}^{2}$. The CdS-Au$\mathrm{TiO}_{2}$ nanorod array photoanode showed a photocurrent as high as $4.07 \mathrm{~mA} / \mathrm{cm}^{2}$ under the same condition (Figure 3.8). The solar-to-chemical energy conversion efficiency $(\eta)$ was calculated for the PEC with the $\mathrm{CdS}-\mathrm{Au}-\mathrm{TiO}_{2}$ nanorod array photoanode. A maximum efficiency of $\sim 2.8 \%$ was reached at $-0.56 \mathrm{~V}$ (vs. $\mathrm{Ag} \mid \mathrm{AgCl})$ under the simulated solar light radiation. The onset potential did not change for the binary CdS$\mathrm{TiO}_{2}$ and the ternary CdS-Au- $\mathrm{TiO}_{2}$ systems. This indicated that the Au nanoparticles, which were fully covered by the CdS QDs layer, did not change the surface chemistry of the photoanode. ${ }^{53}$

The incident photoconversion efficiency (IPCE) spectrum was measured to correlate the energy conversion enhancement with the wavelength of the incident light (Figure 3.8b). Introduction of the Au nanoparticles into the nanorod array enhanced the IPCE substantially in the wavelength range from $325 \mathrm{~nm}$ to $725 \mathrm{~nm}$. The IPCE at $375 \mathrm{~nm}$ was $85 \%$ and 52\% for the nanorod arrays with and without Au nanoparticles, respectively. Figure 3.8c shows the IPCE enhancement factor as a function of the wavelength of incident light, which was obtained by dividing the IPCE value of $\mathrm{CdS}-\mathrm{Au}-\mathrm{TiO}_{2}$ nanorod array with that of CdS- $\mathrm{TiO}_{2}$ nanorod array at a given wavelength. There were two distinct enhancement wavelength regions in Figure 3.8c. In the wavelength range of 325-525 nm, the IPCE enhancement factor remained a constant of 1.5 . In the wavelength range of 525-725 nm, the IPCE enhancement factor increased significantly in the region of the plasmon. Its value was 5.8 at $625 \mathrm{~nm}$ and became 7 at $650 \mathrm{~nm}$ with a high tail at longer wavelengths. The high tail after $650 \mathrm{~nm}$, represented by the dotted line, was an artifact of the division process due to the very low IPCE value (almost zero) of the $\mathrm{CdS}-\mathrm{TiO}_{2}$ photoanode at higher wavelengths (Figure 3.8c).

Introduction of $\mathrm{Au}$ nanoparticles into the semiconductor photoelectrodes has been previously reported 
to improve the performance of photoelectrodes by two possible mechanisms: (i) formation of a Z-scheme and (ii) negative shift of the Fermi level of Au-semiconductor heterostructure. ${ }^{54,55}$ In the present study, the $\mathrm{CdS}-\mathrm{Au}-\mathrm{TiO}_{2}$ nanorod array acted as the photoanode, excluding the possibility of a Z-scheme. When $\mathrm{Au}$ nanoparticles are decorated on the semiconductor photoelectrode surface and are immersed into the liquid electrolyte, a negative shift of the Fermi level in the Au-semiconductor heterostructure occurs due to charge equilibration. ${ }^{54,55}$ This results in a negative shift of both the onset potential in the J-V curve and the flatband potential (apparent Fermi level). ${ }^{79} .80$ In the present work, the onset potential in the J-V curve remained the same after the incorporation of Au nanoparticles into the photoanode, as observed in Figure 3.8a. In addition, the flat-band potential derived from Mott-Schottky plots in Figure 3.8d was -0.97 V (vs. Ag|AgCl) for both $\mathrm{CdS}-\mathrm{TiO}_{2}$ and $\mathrm{CdS}-\mathrm{Au}-\mathrm{TiO}_{2}$. The lack of a negative shift excluded the Fermi-level equilibration as a possible enhancement mechanism. The lack of Fermi level equilibration was not surprising since the gold nanoparticles were fully covered with the CdS QDs layer, which isolated them from the liquid electrolyte.

a

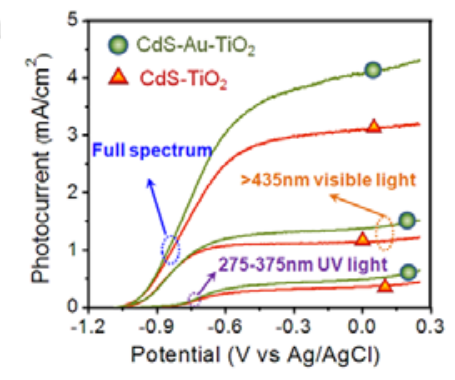

$\mathrm{C}$

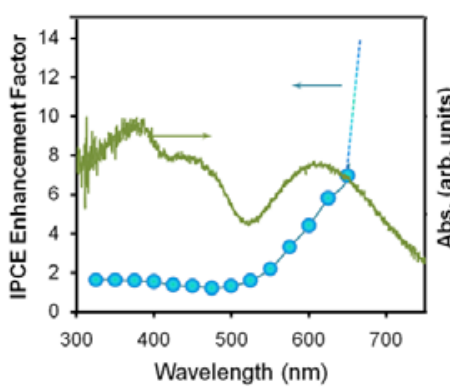

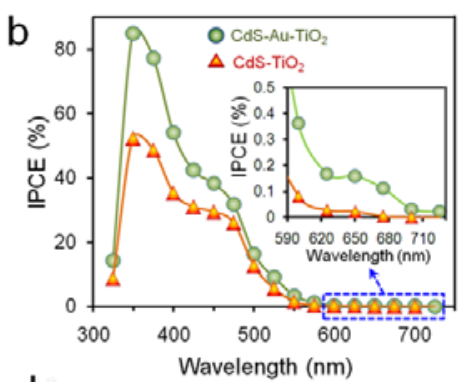

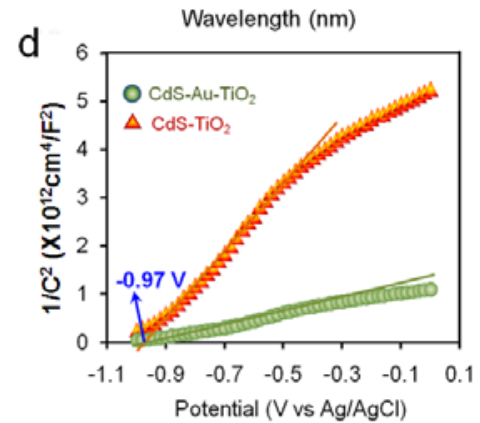

Figure 3.8. Photoelectrochemical performance of CdS-Au- $\mathrm{TiO}_{2}$ nanorod array. a, Photocurrent-applied potential ( $J$ V) curves irradiated by full-spectrum of simulated solar light, visible light (>430 nm), and ultraviolet (275 375 nm), respectively; b, Wavelength-dependent IPCE; c, IPCE enhancement for the CdS-Au- $\mathrm{TiO}_{2}$ nanorod array; d, MottSchottky plots for the $\mathrm{CdS}-\mathrm{TiO}_{2}$ and the $\mathrm{CdS}-\mathrm{Au}-\mathrm{TiO}_{2}$ nanorod array.

Transient-Absorption Analysis of Heterostructures White-light probe transient-absorption spectroscopy was used to understand the various roles of $\mathrm{Au}$ nanoparticles in the heterostructure. The differential absorption of $\mathrm{TiO}_{2}$ was well characterized and exhibited the regions that corresponded to electron- and holetrap state absorptions in the visible region, ${ }^{56-60}$ while CdS showed a decrease in excited-state absorption at the exciton peak exhibiting a bleach. ${ }^{39-46}$ Once an electron-hole pair was excited in the QD, the remaining charge density was reduced and less optical transitions can occur, diminishing the overall absorption (as explained schematically in Figure 3.9). The presence of electron- and hole-trap absorption regions in $\mathrm{TiO}_{2}$ at wavelengths longer than the band gap can be understood as the excited carriers fill the defect states and open the possibility of new optical transitions (Figure 3.10). ${ }^{81-85}$ The spectral position of the defect states is similar to that seen in doped $\mathrm{TiO}_{2}$ (and other metal-oxide semiconductors where occupied mid-gap states exist), which leads to absorption at wavelengths longer than the band-edge. The measured trap state absorption is predominantly assigned to surface states, because the resulting signal is easily suppressed 
using electron and hole scavengers. ${ }^{81-85}$

The excited CdS bleach and $\mathrm{TiO}_{2}$ absorption are shown in Figure 3.10a and Figure 3.10b. The spectral region of the transient-absorption signal for electron-trap states, hole-trap states and free carrier absorption (FCA) have also been shown as defined previously. ${ }^{85}$ In the $\mathrm{CdS}-\mathrm{TiO}_{2}$ heterostructure, the signals from $\mathrm{TiO}_{2}$ and CdS did not overlap spectrally (Figure 3.10c), allowing for the electron- and hole-transfer pathways to be measured independently and the charge-transfer mechanism to be mapped out. This contrasts with the time-resolved fluorescence or single-wavelength transient absorption at the exciton bleach that only measure the CdS relaxation rate. Moreover, FCA only measures the dynamics of carriers once they have transferred into the bulk of the $\mathrm{TiO}_{2}$. Hence, white-light transient absorption provides thorough insight into the charge transport out of the CdS QDs and charge transfer through the interfacial surface states concurrently, which is essential for understanding the long-lived charge transfer necessary for efficient PEC.

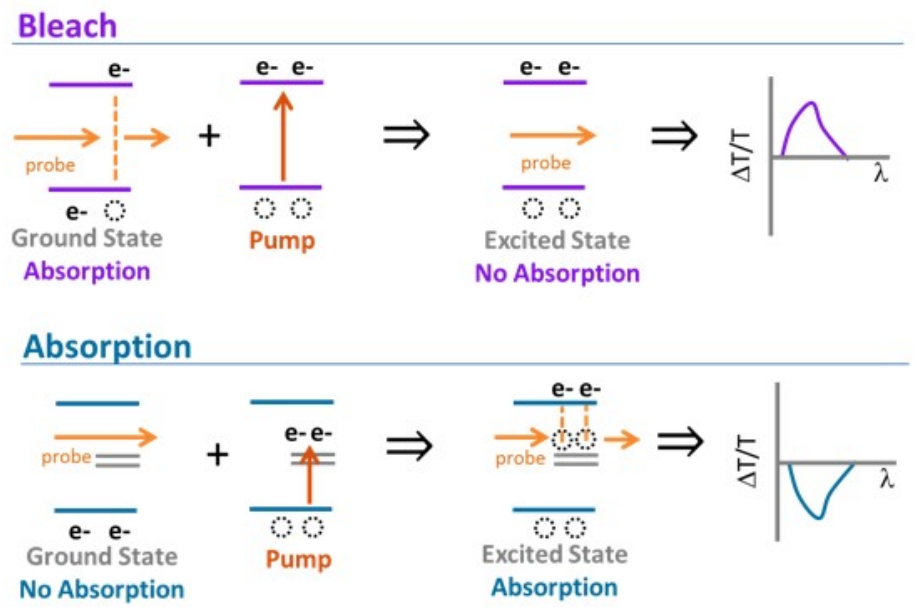

Figure 3.9. Sign of signal in transient absorption. If a semiconductor is probed near the band edge or an absorption band, after excitation, less carriers will be present. This leads to a decrease in the overall absorption of the probe, and the differential transient absorption signal is negative (or the differential transmission is positive). This is known as a bleach. The bleach has a spectral form given by the product of the density of states and quasi-Fermi level under excitation, meaning the bleach is usually similar in position to the ground state absorption. If the probed state is unoccupied before excitation and the excitation wavelength pumps carriers directly into the band, the resulting differential absorption will be positive because more light will be absorbed after excitation (or the differential transmission will be negative). If the state is unoccupied before excitation, the excited state absorption can differ from the ground state absorption, leading to the measurement of absorption at wavelengths shorter than the band edge.

Simple exponential transfer models are insufficient for describing the complex dynamics of the heterostructures, where transport occur from donors, through traps, to acceptors and then back-transfer, with each material having its own average lifetimes. Outside of the low injection limit, the decay dynamics of semiconductors depend nonlinearly on the excitation density. This dependence further precludes a simple exponential solution to the two-level rate equation describing the conduction and valence band of a single semiconductor, let alone the coupled rate equation governing the charge transfer in the heterostructure. Although analytical solutions exist to nonlinear rate equations, the mechanism must be known before hand to select the appropriate model, as several possible models will often accurately describe the decays. Therefore, multiple exponential fits are traditionally used to extract the charge transfer time from the experimental pump-probe data since despite not being a direct solution to the decay kinetics, a fit to the data is almost always possible. While comparison of multiple exponential fitting parameters has been used 
to determine the change in average lifetime with heterostructuring, the loss of a direct connection to the underlying rate equation and the indeterminate nature of exponential fitting leads to a lack of mechanistic details and a large range of reported charge transfer values.
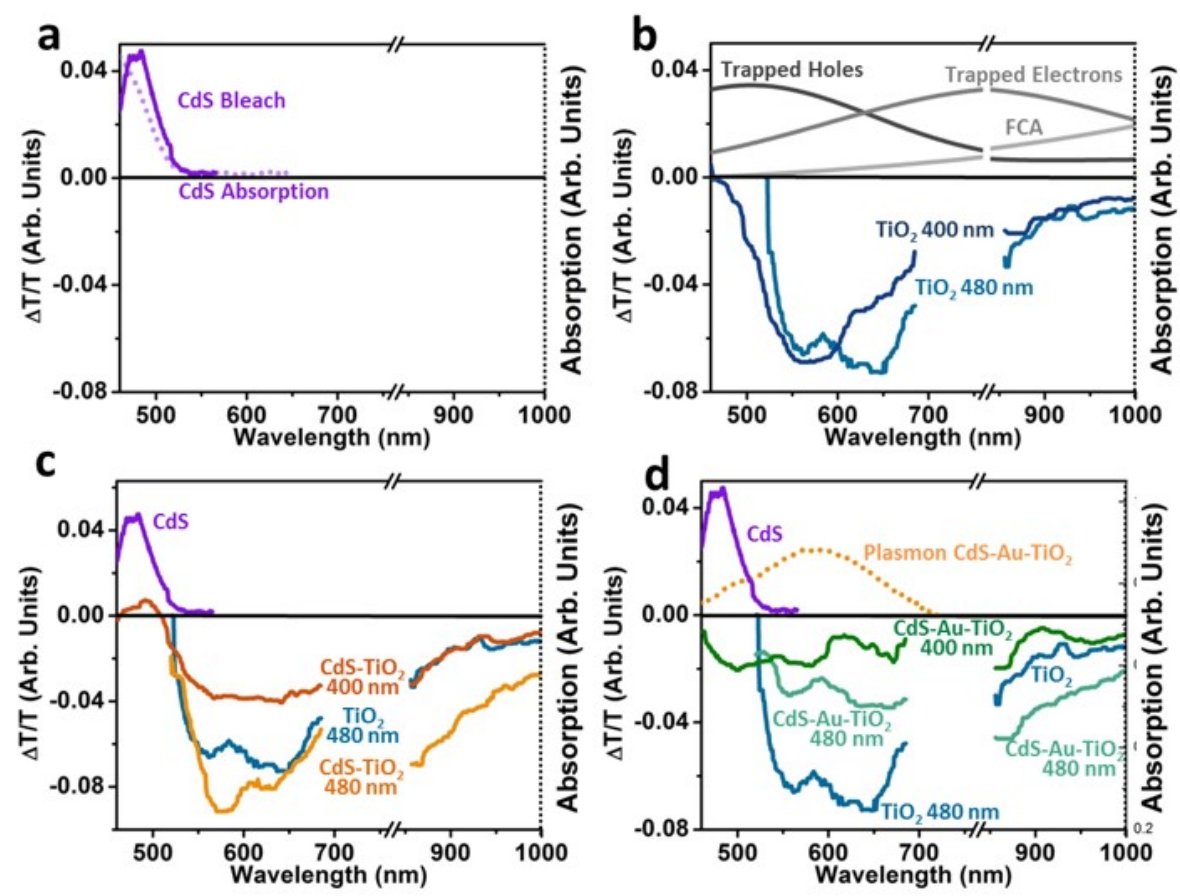

Figure 3.10. Full spectrum transient absorption at $20 \mathrm{ps}$ after excitation. a, Transient absorption for CdS alone pumped at $400 \mathrm{~nm}$, showing the bleach of the exciton. $\mathbf{b}$, The transient absorption for $\mathrm{TiO}_{2}$ shows excited state absorption bands below the band edge. These correspond to trapped holes, trapped electrons, and free carrier absorption as outlined in Ref. 85. c, In the CdS- $\mathrm{TiO}_{2}$ heterostructure the $\mathrm{TiO}_{2}$ excited state absorption and CdS bleach is separate, allowing the observation of both electron and hole transfer pathways. At $480 \mathrm{~nm}$ excitation the pump wavelength is too close to the white light spectrum edge, so the CdS bleach cannot be observed. $\mathbf{d}$, The CdS bleach cannot be observed in the CdS-Au- $\mathrm{TiO}_{2}$, and instead an excited state absorption is seen in the same spectral region.

To overcome these problems, we have adapted a data inversion analysis method as first proposed by Linnros $^{71-73}$ for single semiconductor systems using FCA, which does not rely on average lifetimes. The underlying principles and full procedure are discussed in Methods. Briefly, instead of extracting the lifetime from a fit of the transient-absorption decay, the derivative of the transient-absorption data $\left(\frac{d N}{d t}\right)$ is taken numerically, inverting the measured data from the solution of the underlying rate equation back into the rate equation itself. If the donors and acceptors are both measured and analyzed in this manner, the governing rate equation for charge transfer in the coupled system is directly mapped out, revealing the underlying mechanism. Further, by dividing $\left(\frac{d N}{d t}\right)$ by the carrier density, the instantaneous lifetime is found independent of a fitting procedure. Both the instantaneous lifetime and carrier-relaxation rate have a characteristic dependence on carrier density that reveals the recombination mechanism, see Figure 3.3 and 3.4.

\subsubsection{Discussion}

Mechanism of charge transfer in $\mathrm{CdS}-\mathrm{TiO}_{2}$ Figure 3.11 shows the transient absorption decays measured. The inverted transient-absorption data is shown in Figure 3.11b and 3.11d for the CdS- $\mathrm{TiO}_{2}$ heterostructure 
for 400 and $480 \mathrm{~nm}$ excitation with probe wavelengths from the white light supercontinuum selected to extract the response for the electron-trap states, the hole-trap states in $\mathrm{TiO}_{2}$ and the exciton in CdS. Data from CdS and $\mathrm{TiO}_{2}$ alone are shown for reference. The band alignment and expected electron/hole flow is shown in Figure 3.11c, estimated from a coupled Poisson and drift-diffusion calculation. ${ }^{86}$ The difficulty in interpreting the exponential decays is mitigated once the data are inverted. On a log-log scale, a constant lifetime in the underlying rate equation shows up as a straight line with the linear dependence on the excited density and intercept corresponding to the inverse of the lifetime. A shift up or down of this line and the corresponding change in intercept indicates an increase or decrease in the rate, respectively. A change in slope of the derivative verse the carrier density corresponds to the nonlinear order of the nonlinear relaxation, as shown in Figure 3.3, which is unique for each possible relaxation mechanism.

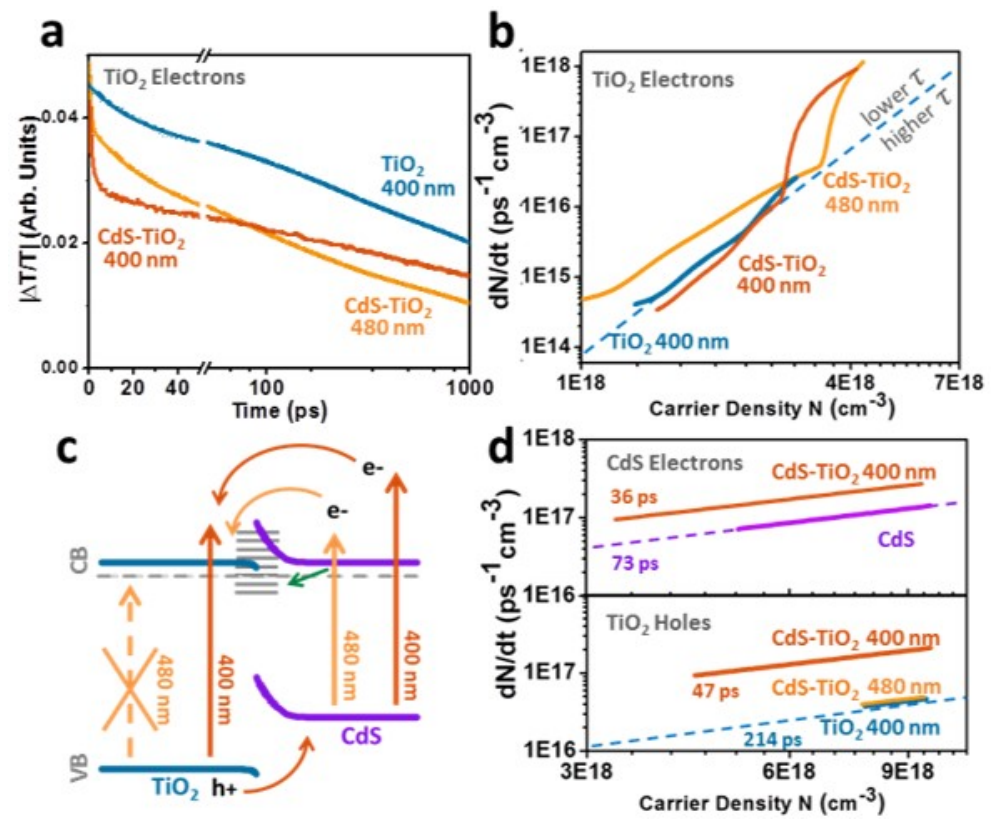

Figure 3.11. Relaxation dynamics in the Cds- $\mathrm{TiO}_{2}$ heterostructure. a, Transient absorption decay dynamics for $\mathrm{TiO}_{2}$ alone and $\mathrm{CdS}-\mathrm{TiO}_{2}$ with 400 and $480 \mathrm{~nm}$ excitation while probing the electron-trap states in $\mathrm{TiO}_{2}$. b, The inverted transient-absorption decay shows the underlying form of the rate equation in the acceptor. c, The charge transfer in the heterostructure can only be described if trap-state Auger scattering is included, green arrow. d, The decrease in lifetime of the CdS probed at the band-edge bleach and $\mathrm{TiO}_{2}$ holes probed at the hole-trap states absorption is consistent with charge transfer out of the donor in the heterostructure. No change is seen for $480 \mathrm{~nm}$ excitation, consistent with no mobile holes being excited in the $\mathrm{TiO}_{2}$.

The inverted data for the CdS-associated electrons and the $\mathrm{TiO}_{2}$-associated trapped holes in the CdS$\mathrm{TiO}_{2}$ heterostructure show a decrease in lifetime (increase in rate) of the donors when pumped at $400 \mathrm{~nm}$ (Figure 3.11d). This reveals the carrier loss from the donors is described by a constant term like in a firstorder rate equation on the timescales shown. Both decays are non-exponential on longer timescales, however the instantaneous lifetimes can be found by transforming the data to the original rate equation independent of fitting. Since the rate is a constant in the range of transfer, the instantaneous lifetime will be unaffected by changes in the timescale, allowing the transfer rate to be calculated. The transfer rates, found from the constant shift in lifetime before and after heterostructuring, are 70 ps and 60 ps for the CdS and $\mathrm{TiO}_{2}$ hole-trap states for $400 \mathrm{~nm}$ excitation, respectively. These values are in agreement with the range of previously published data. ${ }^{59-66}$ No change is seen in the hole lifetime for a $480 \mathrm{~nm}$ pump because although $480 \mathrm{~nm}$ light excites carriers directly into the hole traps in $\mathrm{TiO}_{2}$ creating a transient absorption signal, these carriers are not mobile and are not energetically favorable for charge transfer due to their location mid gap. 
The CdS exciton bleach is also not measured for $480 \mathrm{~nm}$ excitation due to the proximity of the pump to the band edge. The transfer times are summarized in Table 3.1 and 3.2.

Table 3.1. Fitting parameters for transfer model

\begin{tabular}{|c|c|c|c|c|}
\hline Sample & Pump & $\begin{array}{l}\text { Trap-State } \\
\text { Auger Rate }\left(\mathrm{cm}^{3} \mathbf{p s}^{-1}\right)\end{array}$ & Couple Lifetime (ps) & Donor Lifetime (ps) \\
\hline $\mathrm{CdS}^{-\mathrm{TiO}_{2}}$ & $400 \mathrm{~nm}$ & $5.3 \times 10^{-20}$ & 200 & 500 \\
\hline \multirow{3}{*}{$\begin{array}{l}\text { CdS-Au- } \\
\mathrm{TiO}_{2}\end{array}$} & $480 \mathrm{~nm}$ & $1.7 \times 10^{-19}$ & 70 & 200 \\
\hline & $400 \mathrm{~nm}$ & $5.9 \times 10^{-20}$ & 70 & 500 \\
\hline & $480 \mathrm{~nm}$ & $6.5 \times 10^{-20}$ & 70 & 500 \\
\hline
\end{tabular}

Table 3.2. Change in short time scale lifetime of the donors with heterostructuring. The $480 \mathrm{~nm}$ excited, trapped hole $\mathrm{TiO}_{2}$ lifetime had decaying and growing components. The $400 \mathrm{~nm}$ CdS electrons in $\mathrm{CdS}-\mathrm{Au}-\mathrm{TiO}_{2}$ did not have a bleach at the CdS peak due to the mixing between Au and CdS.

\begin{tabular}{|c|c|c|c|c|}
\hline Probe & Sample & Pump & Lifetime (ps) & Transfer Time (ps) \\
\hline Trapped & $\mathrm{TiO}_{2}$ & $400 \mathrm{~nm}$ & 214 & ---- \\
\hline \multirow[t]{5}{*}{ Holes } & & $480 \mathrm{~nm}$ & 193 & ---- \\
\hline & CdS-TiO ${ }_{2}$ & $400 \mathrm{~nm}$ & 47 & 60 \\
\hline & & $480 \mathrm{~nm}$ & 195 & $\sim 0$ \\
\hline & CdS-Au-TiO & $400 \mathrm{~nm}$ & 38 & 46 \\
\hline & & $480 \mathrm{~nm}$ & N/A & N/A \\
\hline CdS & CdS & $400 \mathrm{~nm}$ & 73 & -- \\
\hline \multirow[t]{2}{*}{ Electrons } & CdS-TiO ${ }_{2}$ & $400 \mathrm{~nm}$ & 36 & 71 \\
\hline & CdS-Au-TiO ${ }_{2}$ & $400 \mathrm{~nm}$ & N/A & N/A \\
\hline
\end{tabular}

Next, the more complicated acceptor data was examined. The inverted data is shown for the $\mathrm{TiO}_{2}$ electron-trap states in Figure 3.11b, with the dotted line extrapolating the $\mathrm{TiO}_{2}$ decay. Rates above this line correspond to shorter lifetimes and below correspond to longer lifetimes, with the slope verses carrier density once again determining the nonlinear order of the relaxation mechanism. For $400 \mathrm{~nm}$ and $480 \mathrm{~nm}$ excitation, the initial relaxation rates reveal a faster electron transfer than in $\mathrm{TiO}_{2}$ alone that is more similar to the faster charge transfer times determined for the CdS exciton. At long timescale with $400 \mathrm{~nm}$ pump, the carrier lifetimes are greater than in $\mathrm{TiO}_{2}$ alone (below the dotted line), indicative of the increased charge separation expected in a heterostructure with equal electron and hole transfer. For $480 \mathrm{~nm}$ excitation, only electron transfer from the CdS exists (i.e. no hole transfer as seen in Figure 3.11d), creating a charge imbalance and back transfer dynamics, as reflected in the decrease in long-timescale lifetime (Figure 3.11b).

The constant change in rates and lifetimes measured for the $\mathrm{CdS}$ and $\mathrm{TiO}_{2}$ hole donor states suggest the transfer mechanism is first order (illustrated by curved arrows in Figure 3.11c). The inverted data for the $\mathrm{TiO}_{2}$ acceptor states, however, shows that the initial transfer into the metal-oxide acceptor is faster than the lifetime of $\mathrm{TiO}_{2}$ alone. This result is contrary to a first-order rate-equation model (Figure 3.5) and instead suggests a nonlinear dependence of the initial transfer. Nonlinear transfer behavior explains the apparent disagreement between extracted charge-transfer times when measured from the donor and the acceptors separately, since the acceptor decay dynamics occur at a different rate than the change in the donor's 
lifetime. The upward trend in the rate can only be replicated if a second-order relaxation term is included in the rate equation of the acceptor (Figure 3.5), which corresponds to either radiative relaxation or trapstate Auger scattering (Figure 3.3). Given the long radiative lifetime of $\mathrm{TiO}_{2}$, the relaxation mechanism can be assigned to trap-state Auger scattering. ${ }^{71-73}$ In trap-state Auger scattering an electron relaxes from the CB to a trap state, with the relaxation occurring by giving the excess energy to second electron in the CB. In contrast, Auger scattering occurs by an electron in the CB and a hole in the VB recombining by giving their energy to a second electron or hole. Since the trap state density is constant, trap-state Auger scattering is second-order in carrier density compared to third order in Auger scattering.

The fact that trap-based Auger scattering is a critical part of carrier transfer is not surprising since trap states are known to dominate relaxation dynamics in the heterostructure. ${ }^{87}$ When an organic dye is coupled to a semiconductor, the discrete nature of the molecular electronic structure allows the coupling to be well determined by Marcus theory, and a first-order coupled rate equation is frequently applicable to the resulting decay dynamics since the molecular structure of the organic dye prevents the addition of a large density of surface states. On the other hand, the semiconductor nature of the QDs make the formation of a large density of surface states highly likely, even if the metal-oxide semiconductor is crystalline. ${ }^{87}$ The high density of interfacial states will control the charge transfer rate into the bulk of the $\mathrm{TiO}_{2}$. Meaning that the decay rate of the exciton bleach is first order into the interfacial trap states, but the remaining dynamics are controlled nonlinearly by subsequent transfer from the interfacial states into the bulk of the semiconductor, as represented by the green arrow in Figure 3.11c. This transfer process destroys the simple first-order rate equation picture. The introduction of a nonlinear charge-transfer mechanism with trap states is consistent with results from organic dyes, where the introduction of a large density of defect states in the metal-oxide semiconductor was found to render the measured kinetics indescribable by a first-order coupled rate equation. ${ }^{76,87}$
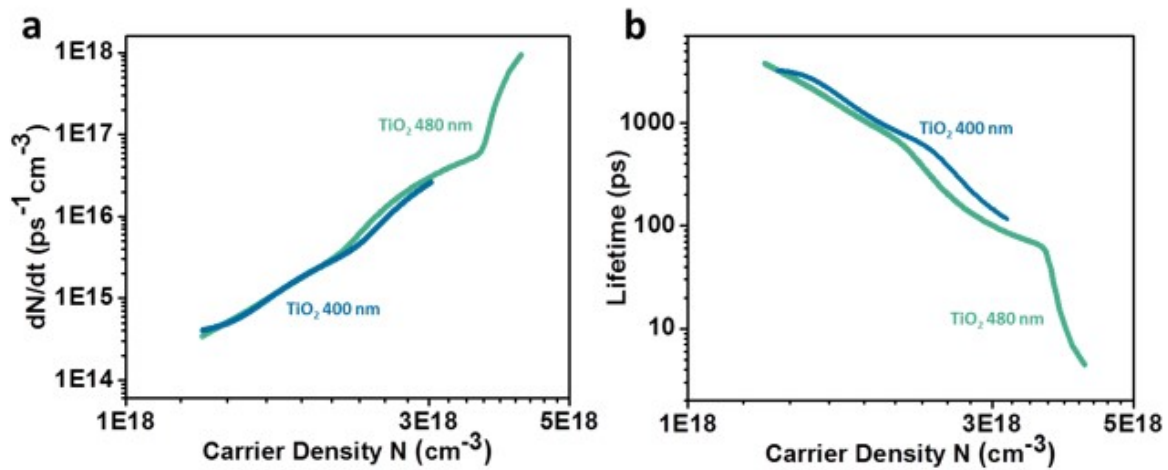

Figure 3.12. Instantaneous lifetime and rate verses carrier density for band edge excitation and direct pumping in $\mathrm{TiO}_{2}$, showing that trap-state Auger dynamics dominate when the trap states are directly pumped.

Trap-state Auger scattering is the dominant mechanism because the increased carrier flow during charge transfer and the increased charge density due to band bending raise the local carrier density in the surface states. This means that even though carrier density remains low in the bulk of the semiconductor and that the relaxation is first order, the charge density at the interface is sufficiently high for a nonlinear recombination mechanism like trap-state Auger scattering to dominate. This phenomenon can be further confirmed by directly pumping $\mathrm{TiO}_{2}$ at long wavelengths below the band edge energy (Figure 3.12) and directly filling the trap states, with the resulting rates again only describable by trap-state Auger scattering and not a third-order nonlinear process, such as conventional Auger scattering (Figure 3.13). Trap-state Auger scattering is consistent with recent reports of diffusion-based Auger transfer and recombination. ${ }^{67,68}$ 

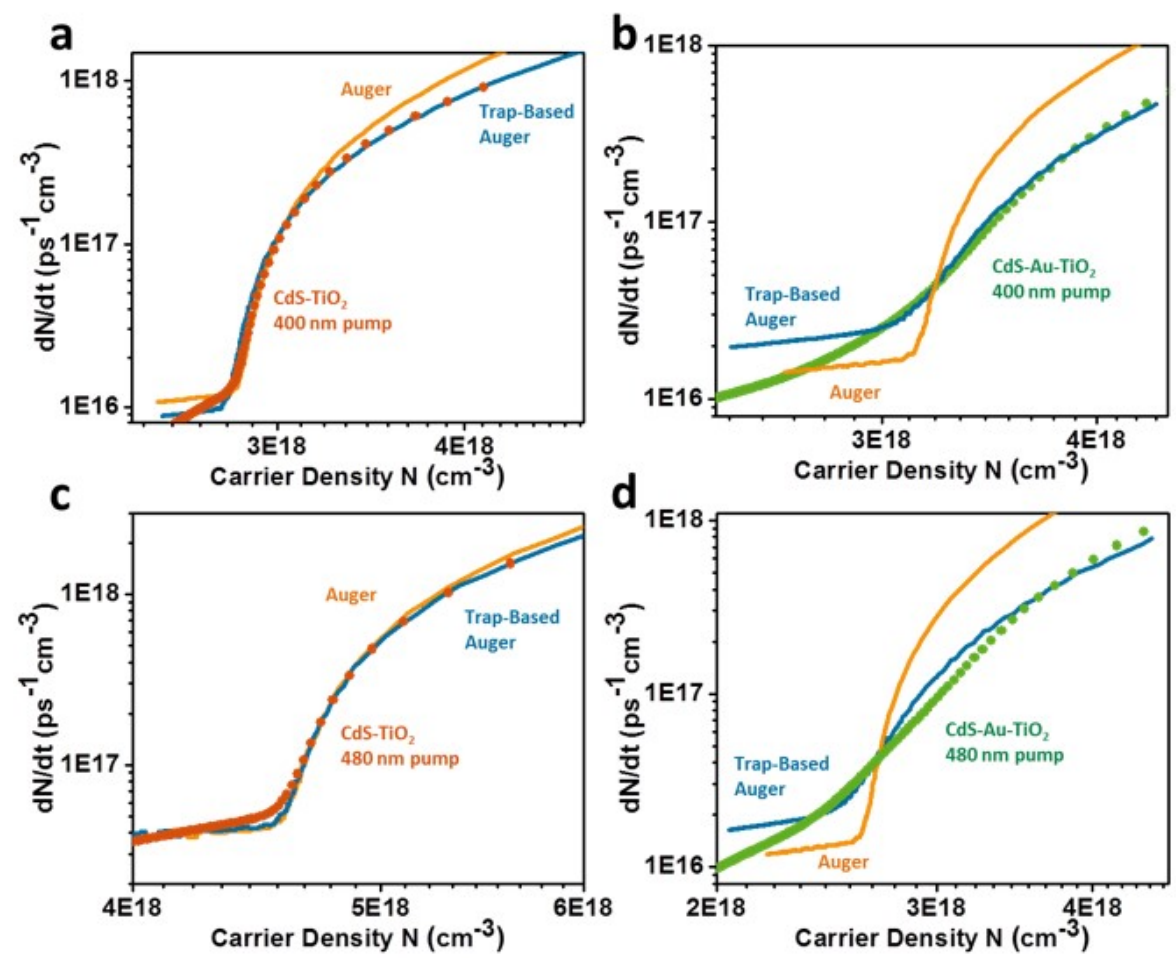

Figure 3.13. Fitting the trap-state Auger coupled rate equation to the decay dynamics in the $\mathrm{TiO}_{2}$ electron trap states for CdS- $\mathrm{TiO}_{2}$ under a, $400 \mathrm{~nm}$ and c, $480 \mathrm{~nm}$ excitation and $\mathrm{CdS}-\mathrm{Au}-\mathrm{TiO}_{2}$ under $\mathbf{b}, 400 \mathrm{~nm}$ and d, $480 \mathrm{~nm}$ excitation, which correspond to excitation of the $\mathrm{CdS}$ and $\mathrm{TiO}_{2}$ or $\mathrm{CdS}$ alone, respectively. In all cases Auger scattering, a third order process, can not fit the data, only a second order process like trap state Auger scattering fits. The $\mathrm{CdS}-\mathrm{Au}-\mathrm{TiO}_{2}$ can not be completely fit using the simple model due to the different trap state filling and dynamics caused by the Ausemiconductor interaction. ${ }^{77,80}$ The fit parameters are in Table 3.1. The curves in Figure 3.13 are not shifted for comparison to ensure accuracy of the fit parameters.

The model for the trap-state Auger transfer mechanism is outlined in Figure 3.14. There are three key parameters: First is the trap-state Auger-scattering time, which determines the curvature of the initial transfer rate (Figure 3.14a and 3.14d). Second is the lifetime of the donor relative to the acceptor, which modifies the rate at the end of transfer from that of the acceptor alone (Figure 3.14b and 3.14e). The lifetime used for the donor is for the last set of states feeding the acceptor. This means that if the QD has surface states, the effective donor lifetime will be that of the surface states and not that of the measured QD bleach. The effective donor lifetime is determined by the transit time from the bulk QD states to the acceptor states. Finally is the transfer rate, which increases the transfer time or even adds in a second transfer curvature in the rate if it is shorter than the trap-state Auger-scattering (Figure 3.14c and 3.14f). To fit the $400 \mathrm{~nm}$ and $480 \mathrm{~nm}$ data, the coupling time is initially fixed at $70 \mathrm{ps}$ as determined by the change in the CdS lifetime. For $480 \mathrm{~nm}$ excitation, the trap-state Auger scattering rate is higher, $1.7 \times 10^{-19} \mathrm{~cm}^{3} \mathrm{ps}^{-1}$ to $5.3 \times 10^{-20} \mathrm{~cm}^{3} \mathrm{ps}^{-1}$ for $400 \mathrm{~nm}$ excitation, which is indicative of a faster relaxation. The effective lifetime of the donor differs between the pump wavelengths, represented by the low carrier-density rate after the transfer is complete. Additionally, the effective donor lifetime is $200 \mathrm{ps}$ for $480 \mathrm{~nm}$ compared to $500 \mathrm{ps}$ at $400 \mathrm{~nm}$. A summary of the fitted parameters is shown in Table 3.1.

The wavelength-dependent change in fitted parameters can be understood in terms of the scheme shown in Figure 3.11c and based on the spectral distribution of transferred carriers presented in Figure 3.10. For $480 \mathrm{~nm}$ excitation, the carriers are excited at the CB edge of CdS and transfer into the $\mathrm{TiO}_{2}$ trap states, 
which creates a higher electron density, a faster transfer time, increased trap-state Auger rate and reduced effective donor lifetime. For $400 \mathrm{~nm}$ excitation the carriers are created in the QD CB continuum, then transfer to the $\mathrm{TiO}_{2} \mathrm{CB}$ and trap states, which slows the transfer and leads to a higher effective donor lifetime as observed in Figure 3.11b. The latter process mimics excitation in $\mathrm{TiO}_{2}$ for a $400 \mathrm{~nm}$ pump and is evident by comparing the spectral distribution of transferred carriers (Figure 3.10). To completely fit the data for $400 \mathrm{~nm}$ excitation the coupling time is found to be $200 \mathrm{ps}$ (as compared to 70 ps before heterostructuring), which further supports the increase in the transfer time for $400 \mathrm{~nm}$ excitation compared to the direct transfer for $480 \mathrm{~nm}$ excitation.
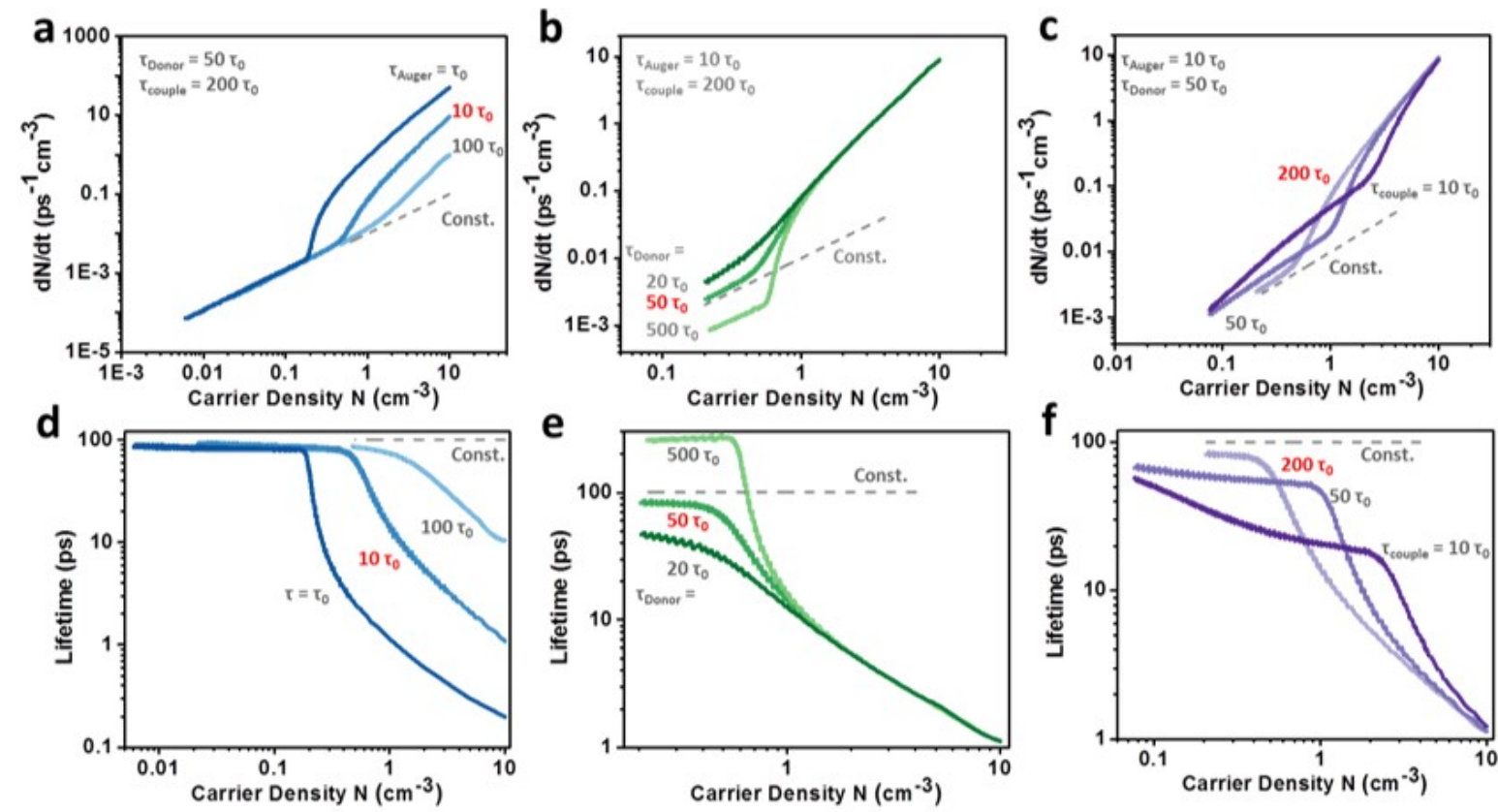

Figure 3.14. The trap state Auger coupled rate equation is given by

$$
\begin{aligned}
& \frac{d N_{\text {Acceptor }}}{d t}=-\frac{N_{\text {Acceptor }} * N_{\text {Acceptor }}}{\tau_{\text {Auger }}}+\frac{N_{\text {Donor }}}{\tau_{\text {couple }}} \\
& \frac{d N_{\text {Donor }}}{d t}=-\frac{N_{\text {Donor }}}{\tau_{\text {Donor }}}-\frac{N_{\text {Donor }}}{\tau_{\text {couple }}}
\end{aligned}
$$

The effect of modulating a, the trap state Auger scattering rate, $\mathbf{b}$, the effective donor lifetime which describes the lifetime as seen by the acceptor, and c, the charge transfer time are shown. The corresponding change in instantaneous lifetime is shown in $\mathbf{d}$ through $\mathbf{f}$. It is seen that the trap state scattering rate modulates the initial curvature, the effective donor lifetime changes the rate after transfer, and the coupling time determines the range over which carriers are transferred.

Mechanism of charge transfer in $\mathrm{CdS}-\mathrm{Au}-\mathrm{TiO}_{2}$ The inverted transient-absorption analysis is next applied to understand the role of the inclusion of $\mathrm{Au}$ in the heterostructure. The inverted exponential decays (Figure 3.15b) shows that the electron-trap states in $\mathrm{TiO}_{2}$ fill quickly followed by a longer decay relative to $\mathrm{TiO}_{2}$ alone. By comparing Figure 3.15b to Figure 3.11b it is seen the presence of the Au affects the response in several respects. Namely, the initial rate at higher carrier densities (above $4 \times 10^{18} \mathrm{~cm}^{-3}$ ) has a less abrupt curvature than in the $\mathrm{CdS}-\mathrm{TiO}_{2}$ and spans a larger range of excited carrier density from $3.9 \times 10^{18} \mathrm{~cm}^{-3}$ up to $7 \times 10^{18} \mathrm{~cm}^{-3}$. The trap-state Auger-scattering rates are $5.9 \times 10^{-20} \mathrm{~cm}^{3} \mathrm{ps}^{-1}$ and $6.5 \times 10^{-20} \mathrm{~cm}^{3} \mathrm{ps}^{-1}$ for the 400 and $480 \mathrm{~nm}$ pumps, respectively. In particular, the rate at $480 \mathrm{~nm}$ excitation is much reduced from CdS$\mathrm{TiO}_{2}$. The effective donor lifetime is found to be $500 \mathrm{ps}$ in both cases, however, this still does not fully account for the rate after transfer is complete (Figure 3.13), suggesting an additional transfer rate from CdS 
to $\mathrm{Au}$ and $\mathrm{Au}$ to $\mathrm{TiO}_{2}$. The additional deviation from the model that develops in the low carrier density regime for the $\mathrm{CdS}-\mathrm{Au}-\mathrm{TiO}_{2}$ suggests that the transfer from $\mathrm{CdS}$ to $\mathrm{Au}$ has been extended and that the transfer from $\mathrm{Au}$ to $\mathrm{TiO}_{2}$ is quicker than the Auger-scattering rate (similar to Figure 3.14c). The transfer range spans a larger carrier density range from $1 \times 10^{18} \mathrm{~cm}^{-3}$ up to $3.8 \times 10^{18} \mathrm{~cm}^{-3}$ than the $\mathrm{CdS}-\mathrm{TiO}_{2}$, which indicates that the $\mathrm{Au}$ is promoting the transfer of a larger number of carriers (i.e. the large span of carrier density span) by extending the transfer process. The observed shifts are consistent with those modelled in Figure 3.14 for an increased transfer rate and reduced trap-based Auger scattering. This result suggests that the $\mathrm{Au}$ is acting as an intermediate "relay" for charge transfer from the $\mathrm{CdS}$ to $\mathrm{TiO}_{2}$ (Figure 3.15c).

Next it is seen that after the transfer from the CdS to the $\mathrm{Au}$ to the $\mathrm{TiO}_{2}$ is complete, the carriers experience a back transfer ended by a long lifetime that approaches or exceeds that of $\mathrm{TiO}_{2}$ alone. Observing medium-timescale equilibration and long-timescale charge trapping is consistent with the chargeequilibration effects measured for metals in contact with semiconductors. ${ }^{77,80}$ Interestingly, the Au nanoparticles remove the long-timescale decrease in lifetime associated with back-transfer in the $\mathrm{CdS}-\mathrm{TiO}_{2}$ for $480 \mathrm{~nm}$ excitation. Without the Au nanoparticles, the measured long-timescale, lower carrier density dynamics were dependent heavily on the pump wavelength (Figure 3.11b). The addition of Au makes the final rate similar at both excitation wavelengths (Figure 3.15b), further confirming that the Au nanoparticles act as a carriers relay from $\mathrm{CdS}$ to $\mathrm{TiO}_{2}$. The increase in number, rate, and lifetime independent of excitation wavelength explains the constant enhancement in the IPCE by the Au nanoparticles in the CdS-Au- $\mathrm{TiO}_{2}$ heterostructure for excitation with wavelengths shorter than $525 \mathrm{~nm}$ (Figure 3.8c).

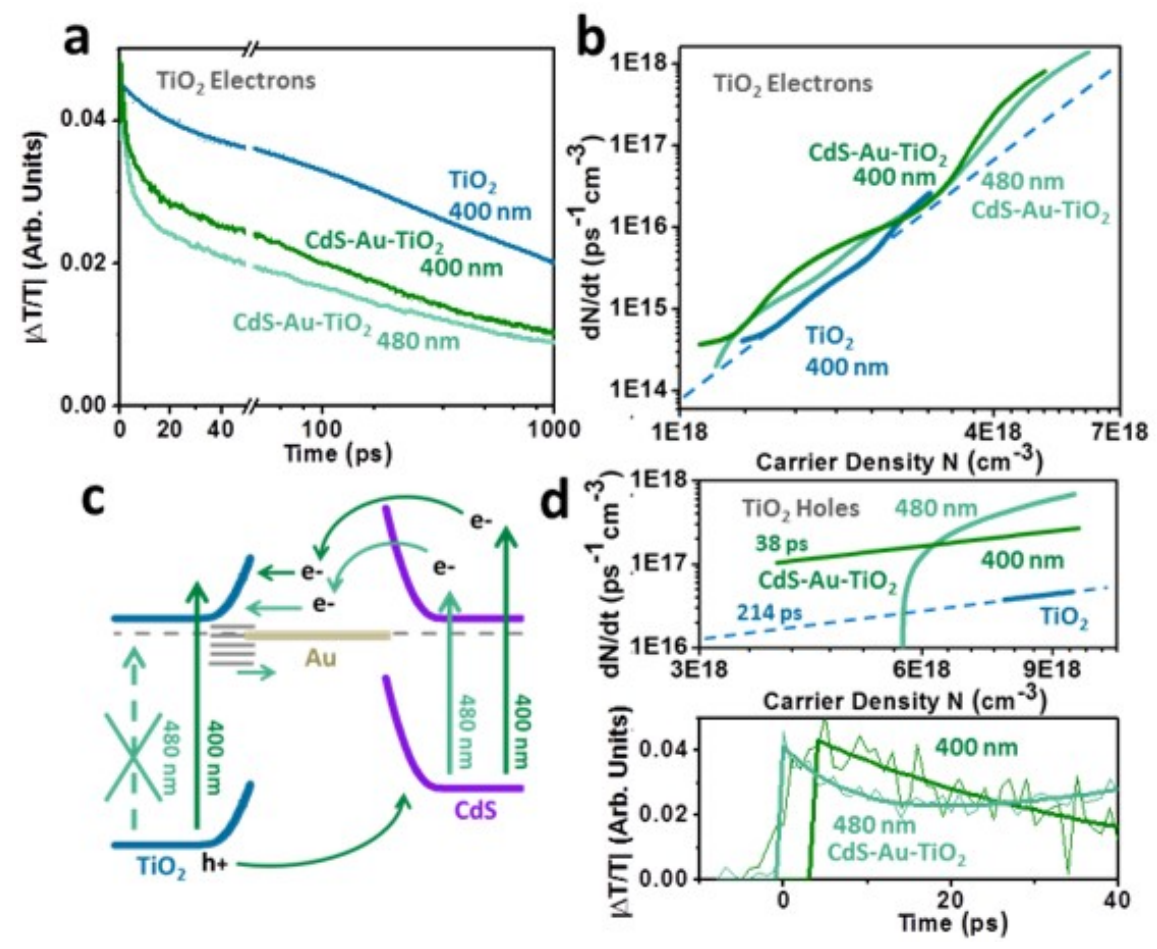

Figure 3.15. Relaxation dynamics in the Cds- $\mathrm{Au}-\mathrm{TiO}_{2}$ heterostructure. a, Transient-absorption decay dynamics for $\mathrm{TiO}_{2}$ alone and CdS-Au-TiO 2 with a 400 and $480 \mathrm{~nm}$ excitation while probing the electron trap states in $\mathrm{TiO}_{2}$. b, The inverted transient-absorption decay showing the underlying form of the rate equation in the acceptor. The trap-state Auger scattering rate is decreased, the coupling time is increased, and the effective donor lifetime is increased compared to $\mathrm{CdS}-\mathrm{TiO}_{2}$, $\mathbf{c}$, proving that the $\mathrm{Au}$ is acting as an electron relay and prolonging the carrier lifetime. $\mathbf{d}$, The decrease in lifetime of the $\mathrm{TiO}_{2}$ holes probed at the hole-trap states absorption is consistent with charge transfer out of the donor in the heterostructure at $400 \mathrm{~nm}$. At $480 \mathrm{~nm}$ the hole states undergo a fast equilibration, consistent with the interfacial Schottky barrier modifying the trap state occupation and dynamics. 
The deviations from the transfer model in the CdS-Au- $\mathrm{TiO}_{2}$ are attributed to $\mathrm{Au}$ modifying the surface states of both $\mathrm{TiO}_{2}$ and CdS. ${ }^{88-94}$ The modification is evident from suppression of the CdS bleach when $\mathrm{Au}$ is added and is consistent with previous findings. ${ }^{89,93}$ The initial analysis supports states in CdS being mixed with (or swamped by) those in the gold, leading to an increased carrier density and increased absorption after the initial excitation is complete. This effect is further illustrated by comparing the rate of carrier relaxation in $\mathrm{Au}-\mathrm{TiO}_{2}$ to $\mathrm{CdS}-\mathrm{Au}-\mathrm{TiO}_{2}$ in Figure 3.16 against the original $\mathrm{TiO}_{2}$ rates, where the shifts indicate a change in interfacial charge density and trap occupation with the addition of $\mathrm{Au} / \mathrm{TiO}_{2} \mathrm{Schottky}$ barrier (Figure 3.15c). The effect of the Schottky barrier is further evidenced in the dynamics of the hole states (Figure 3.15d). For $400 \mathrm{~nm}$ excitation, the hole transfer rate is $46 \mathrm{ps}$ with a long rise time, slightly faster than the system without the Au (Figure 3.11d). However, for $480 \mathrm{~nm}$ excitation, a higher initial relaxation rate followed by a fast back transfer is measured, represented as a divergence in the rate of Figure 3.15d. This behavior is consistent with the lack of hole transfer for 480-nm excitation and instead supports charge equilibration at the metal-semiconductor interface. It is unlikely that the transient absorption signal is from $\mathrm{Au}$ alone because of the small concentration of $\mathrm{Au}$ in the heterostructure and the lack of a measurable plasmon bleach for both 400-nm and 480-nm excitation.
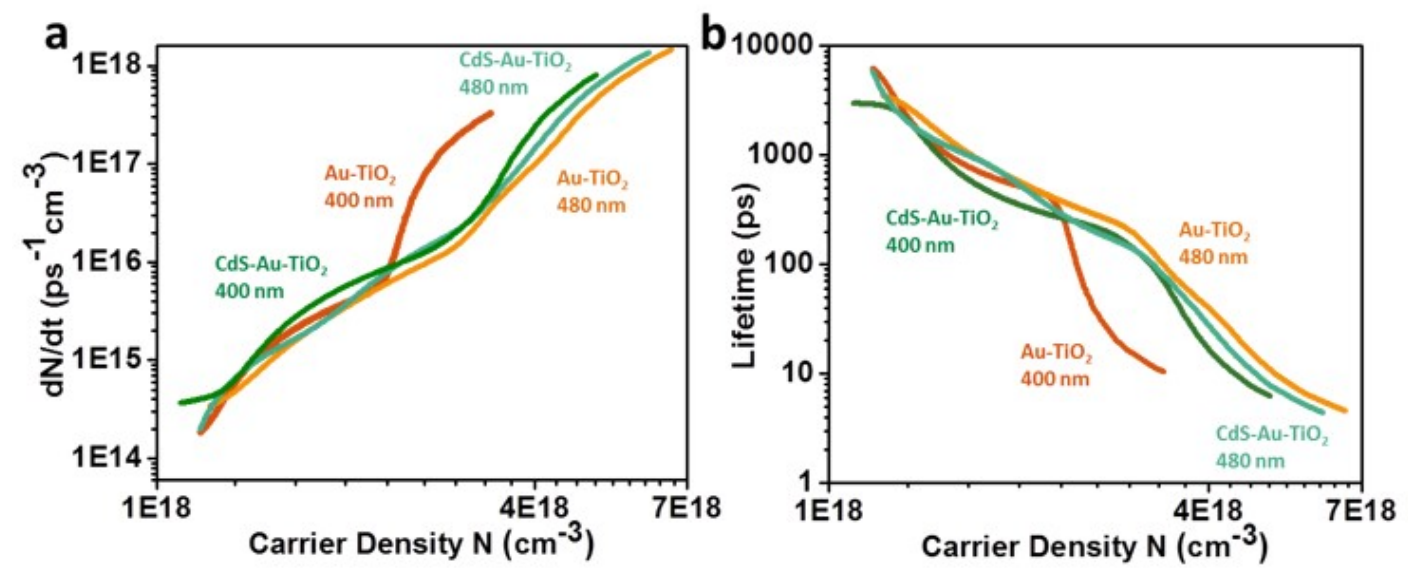

Figure 3.16. Instantaneous lifetime and rate verses carrier density for $\mathrm{Au}-\mathrm{TiO}_{2}$ and $\mathrm{CdS}-\mathrm{Au}-\mathrm{TiO}_{2}$ for $400 \mathrm{~nm}$ and 480 $\mathrm{nm}$ excitation. The dynamics are seen to be modified from pure $\mathrm{TiO}_{2}$ in all cases, with heterostructuring further changing the transfer times. This is consistent with Au acting as an electron sink and shuttle, as well as modifying the interface states.

Mechanism of plasmonic energy transfer from $\mathrm{Au}$ to $\mathrm{TiO}_{2}$ Plasmonic dynamics due to the Au nanoparticles were investigated using a $675 \mathrm{~nm}$ pump as this wavelength can excite the LSPR but not the $\mathrm{CdS}$ or $\mathrm{TiO}_{2}$. Plasmonic transfer is determined by measuring the corresponding rise in $\mathrm{CdS}$ or $\mathrm{TiO}_{2}$ excited state when the plasmon is excited. The charge transfer is similar to that of Figure 3.11 and Figure 3.15, but with the plasmon now acting as the donor and both semiconductors as acceptors, Figure 3.16d. The plasmon in the $\mathrm{Au}-\mathrm{TiO}_{2}$ and CdS-Au-TiO${ }_{2}$ is shifted spectrally, from $550 \mathrm{~nm}$ to $600 \mathrm{~nm}$ in, allowing differentiation between the plasmonic energy transfer mechanisms since PIRET and DET differ according to the spectral overlap and hot-electron distribution, respectively. Figure 3.16b shows that exciting the LSPR leads to strong absorption in the $\mathrm{TiO}_{2}$ electron-trap states only, with a smaller change observed in the cross-over region between trapped holes and trapped electrons. No change is seen in the CdS bleach/absorption region when the LSPR is pumped, with the transient absorption signal replicating $\mathrm{TiO}_{2}$ alone and not CdS-Au$\mathrm{TiO}_{2}$ with a $400 \mathrm{~nm}$ or $480 \mathrm{~nm}$ excitation wavelength. Given that no spectral overlap exists between $\mathrm{TiO}_{2}$ and the LSPR, the plasmonic energy transfer mechanism can only be hot electron transfer by DET. This is further confirmed by the larger percentage of transferred electrons with little change in the trapped hole 
states (Figure 3.16c), as PIRET would have led to an equal number of electron and hole pairs being created through resonant interband transitions in the semiconductor.

DET is proposed to occur by transfer of the hot electrons over the interfacial Schottky barrier (Figure 3.16d). The effects of the Schottky barrier on hot electron transfer can be directly measured by comparing the shift in the excited spectral distribution verse the plasmon shift in the $\mathrm{Au}-\mathrm{TiO}_{2}$ and $\mathrm{CdS}-\mathrm{Au}-\mathrm{TiO}_{2}$. Figure 3.16c shows the transferred carrier distribution in the $\mathrm{CdS}-\mathrm{Au}-\mathrm{TiO}_{2}$ fills the electron trap states higher in wavelength than the $\mathrm{Au}-\mathrm{TiO}_{2}$. The shift in the transferred electron distribution is similar to the plasmon redshift. This occurs because the hot plasmonic electrons in the $\mathrm{Au}-\mathrm{TiO}_{2}$ system have a higher energetic offset from the Au Fermi level due to the blue-shifted plasmon peak, and can therefore more effectively overcome the interfacial Schottky barrier to transfer into the $\mathrm{TiO}_{2} \mathrm{CB}$ (Figure 3.16d). The CdS-Au-TiO 2 has a red-shifted plasmon with a smaller energy offset from the Au Fermi level; it therefore cannot effectively overcome the interfacial Schottky barrier, instead transferring electrons to the trap states in $\mathrm{TiO}_{2}$ below the CB (Figure 3.16d). In neither case do the carriers appear to have enough energy to efficiently transfer into the CdS.

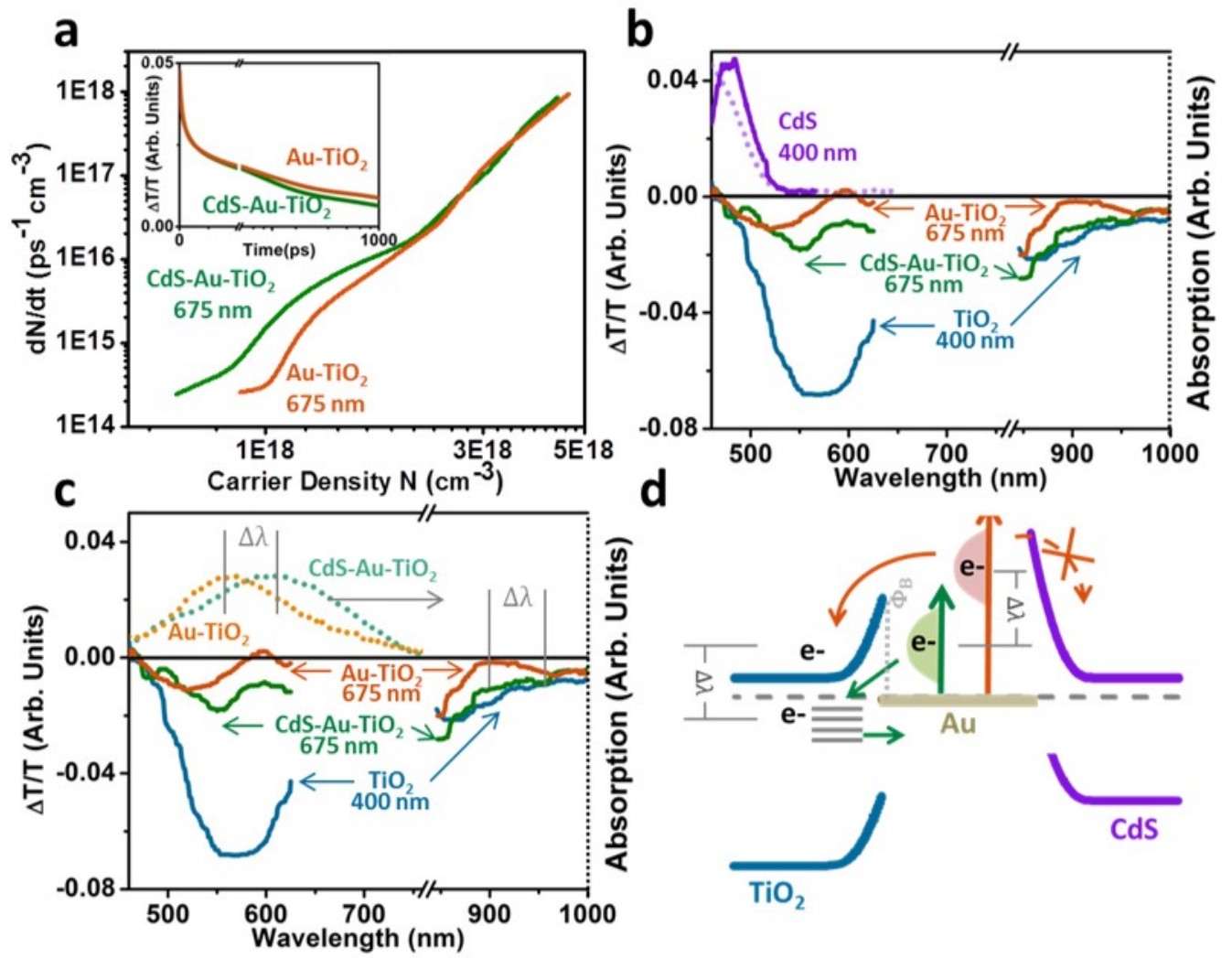

Figure 3.16. Plasmonic energy transfer in CdS-Au- $-\mathrm{TiO}_{2}$. a, Dependence of the long timescale decay rate on the plasmon position relative to the $675 \mathrm{~nm}$ pump. b, The full-spectrum transient-absorption signal at $20 \mathrm{ps}$ after excitation, showing the lack of CdS bleach for $\mathrm{Au}-\mathrm{TiO}_{2}$ or $\mathrm{CdS}-\mathrm{Au}-\mathrm{TiO}_{2}$ which indicates no charge transfer into the CdS. Charge transfer is seen into the $\mathrm{TiO}_{2}$ electron-trap states. c, The shift in the LSPR between $\mathrm{Au}_{-} \mathrm{TiO}_{2}$ and CdS$\mathrm{Au}-\mathrm{TiO}_{2}$ corresponds to the shift in the filling of the $\mathrm{TiO}_{2}$ trap states. d, The change in back transfer dynamics and filling of the $\mathrm{TiO}_{2}$ electron trap states without charge transfer into CdS is explained by the energy of the hot plasmonic electron distribution relative to the interfacial Schottky barrier, $\Phi_{\mathrm{B}}$.

The effect of offset hot electron energy from the Schottky barrier is also seen in the inverted transientabsorption data (Figure 3.16a). The electron transfer is similar on short timescales for both heterostructures. However at long timescale, the carriers in the $\mathrm{CdS}-\mathrm{Au}-\mathrm{TiO}_{2}$ have a shorter lifetime because they can transfer into the interfacial trap states and quickly transfer back into the $\mathrm{Au}$. In comparison, the $\mathrm{Au}-\mathrm{TiO}_{2} \mathrm{Plasmonic}$ electrons have a longer lifetime because they can become trapped behind the interfacial Schottky barrier. 
The back transfer in the CdS-Au- $\mathrm{TiO}_{2}$ may explain the small increase in the overall IPCE despite the large increase in absorption due to the plasmon. If the plasmonic electrons are to be used more efficiently, the transferred charge carriers need to be trapped behind the Schottky barrier so they can be extracted before recombination.

Dual role of Au nanoparticle The inversion analysis method for transient-absorption spectroscopy has revealed the critical role that the interfacial trap states play in the $\mathrm{CdS}-\mathrm{TiO}_{2}$, the effect of $\mathrm{Au}$ on interfacial transfer and long-timescale back transfer, and the effect of the plasmonic hot electron energy relative to the interfacial Schottky barrier. Using this information, the PEC performance can be accurately described by Figure 3.17.

At incident light wavelengths shorter than $525 \mathrm{~nm}$, the LSPR in the Au nanoparticles is not excited. Hence the photocurrent enhancement is not due to the LSPR of Au nanoparticles. Instead, charge carriers are created in the CdS QDs. The photogenerated charge carriers transfer from the CdS QDs to $\mathrm{TiO}_{2}$ via the Au nanoparticles as shown in Figure 3.17a. At wavelengths longer than $525 \mathrm{~nm}$, the energy of the light is insufficient to create carriers in the $\mathrm{TiO}_{2}$ or CdS, hence no charge transfer occurs from CdS to $\mathrm{TiO}_{2}$. However, the LSPR is excited in the Au nanoparticles. When the plasmonic Au nanoparticles are in intimate contact with $\mathrm{TiO}_{2}$, hot electrons are excited and can transfer from the plasmonic metal to the conduction band of $\mathrm{TiO}_{2}$ as shown in Figure 3.17b. In this case, the $\mathrm{Au}$ nanoparticles act as the plasmonic photosensitizer, increasing photoconversion in the wavelength from $525 \mathrm{~nm}$ to $725 \mathrm{~nm}$. This is confirmed by the variation in IPCE and IPCE enhancement factor at wavelengths corresponding to the LSPR from $525 \mathrm{~nm}$ to $725 \mathrm{~nm}$.
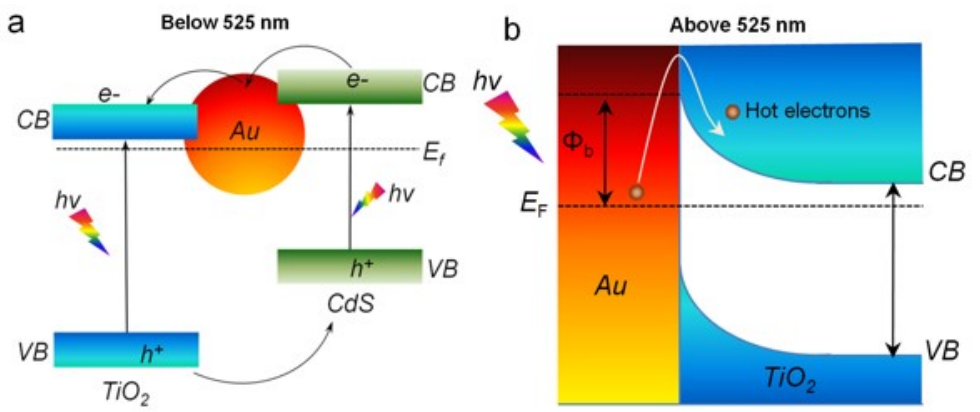

Figure 3.17. The wavelength-dependent dual role of Au nanoparticles in the CdS-Au-TiO2 sandwich structure. a, Electron relay effect of Au nanoparticles, facilitating the charge transfer from CdS QDs to $\mathrm{TiO}_{2}$ nanorod under the irradiation of incident solar light with the wavelength $<525 \mathrm{~nm}$. b, Plasmonic energy transfer from the excited $\mathrm{Au}$ nanoparticles to $\mathrm{TiO}_{2}$ through the hot electrons transfer under the irradiation of incident solar light with the wavelength $>525 \mathrm{~nm}$. $\mathrm{CB}=$ Conduction band, $\mathrm{VB}=$ Valence band, $\mathrm{E}_{\mathrm{f}}=$ Fermi energy level, and $\Phi_{\mathrm{B}}=$ Schottky barrier.

\subsection{Chapter Summary}

Plasmonic hot carrier transfer is promising for enhancing below-band edge photoconversion in wide band gap semiconductors. By changing the metal nanoparticle size, shape, geometry, and constituent metal the energy of hot electrons and holes can be modulated. However, the subsequent charge extraction and equilibration issues have challenged efficient hot carrier extraction for use in a solar energy conversion role. To test the theoretical developments surrounding hot carrier transfer gold nanoparticles were sandwiched between $\mathrm{TiO}_{2}$ nanorods and a CdS QD shell layer. This geometry showed that the gold nanoparticles played a dual role in enhancing the solar-to-chemical energy conversion efficiency of PEC. The function of the Au nanoparticles depended on the incident light wavelength relative to the plasmon. Upon irradiation at 
wavelengths shorter than $525 \mathrm{~nm}$, the charge carriers were generated in the CdS QDs but not in the plasmon. In this case, the Au nanoparticles served as an electron relay of charge carriers between the CdS QDs and $\mathrm{TiO}_{2}$. At the wavelength range of $525 \sim 725 \mathrm{~nm}$, the CdS QDs were unable to serve as a photosensitizer but the LSPR was excited in the Au nanoparticles and acted as a plasmonic photosensitizers, injecting hot electrons into the $\mathrm{TiO}_{2}$. This combination of the charge-transfer enhancement and the light absorption bandwidth extension in a single structure was responsible for the improvement in the solar-to-chemical energy conversion efficiency.

Further, a newly developed inversion transient-absorption analysis method combined with a white-light probe showed the key role that the interfacial trap states and the trap-state Auger recombination played during the processes of charge transfer between $\mathrm{CdS}$ and $\mathrm{TiO}_{2}$. By including Au nanoparticles in the heterostructure, it was found that (i) the rate of trap-state Auger recombination decreased, (ii) the number of charge carriers transferred increased, (iii) the rate of transfer increased and (iv) the charge separation was prolonged, even at the point of suppressing the back transfer when only CdS was excited. The Au nanoparticles were therefore found to eliminate some of the deficiencies caused by interfacial trap sates, which improved the photoelectrochemical performance as seen in the IPCE. For these materials, the plasmonic energy-transfer mechanism was proven to be DET, with the rate of charge transfer and back transfer dependent on the relative energy of the hot plasmonic electrons to the Schottky barrier.

However, it was found that, consistent with predictions, the charge equilibration and charge extraction problems kept the plasmonic hot carrier efficiency less than $1 \%$. The primary role of the gold nanoparticles was instead shown to be improving charge transport between the semiconductors, increasing efficiency but not extending the photoconversion range.

\subsection{References}

1. Walter, M. G.; Warren, E. L.; McKone, J. R.; Boettcher, S. W.; Mi, Q.; Santori, E. A.; Lewis, N. S. Chem. Rev., 2010, 110, 6446-6473.

2. Maeda, K.; Domen, K. J. Phys. Chem. Lett 2010, 1, 2655-2661.

3. Katz, M. J.; Riha, S. C.; Jeong, N. C.; Martinson, A. B. F.; Farha, O. K.; Hupp, J. T. Coord. Chem. Rev. 2012, 256, 2521-2529.

4. Sivula, F.; Formal, L; Grätzel, M. ChemSusChem 2011, 4, 432-449.

5. Reece, S. Y.; Hamel, J. A.; Sung, K.; Jarvi, T. D.; Esswein, A. J.; Pijpers, J. J. H.; Nocera, D. G. Science 2011, 334, 645-648.

6. Abdi, F. F.; Han, L.; Smets, A. H. M.; Zeman, M.; Dam, B.; van de Krol, R. Nat. Commun. 2013, 4, 2195.

7. Hashimoto, K.; Irie, H.; Fujishima, A. Jpn. J. Appl. Phys. 2005, 44, 8269-8285.

8. Ni, M.; Leung, M. K. H.; Leung, D. Y. C.; Sumathy, K. Renew. Sustain. Energy Rev. 2007, 11, 401-425.

9. Thompson, T. L.; Yates, J. T. Chem. Rev. 2006, 106, 4428-4453.

10. Kumar, S. G.; Devi, L. G. J. Phys. Chem. A 2011, 115, 13211-13241.

11. http://www1.eere.energy.gov/vehiclesandfuels/pdfs/program/hptt_roadmap_june2013.pdf (accessed October, 2015).

12. Link, S.; El-Sayed, M. A. Int. Rev. Phys. Chem. 2000, 19, 409-453.

13. Sönnichsen, C.; Franzl, T.; Wilk, T.; von Plessen, G.; Feldmann, J.; Wilson, O.; Mulvaney, P. Phys. Rev. Lett. 2002, 88, 077402. 
14. Link, S.; El-Sayed, M. A. J. Phys. Chem. B 1999, 103, 4212-4217.

15. Link, S.; El-Sayed, M. A. J. Phys. Chem. B 1999, 103, 8410-8426.

16. Govorov, A. O.; Zhang, H.; Gun'ko, Y. K. J. Phys. Chem. C 2013, 117, 16616-16631.

17. Sundararaman, R.; Narang, P.; Jermyn, A. S.; Goddard III, W. A.; Atwater, H. A. Nat. Commun. 2014, 5, 5788.

18. Manjavacas, A.; Liu, J. G.; Kulkarni, V.; Nordlander, P. ACS Nano 2014, 8, 7630-7638.

19. Bernardi, M.; Mustafa, J.; Neaton, J. B.; Louie, S. G. Nat. Commun. 2015, 6, 7044.

20. Govorov, A. O.; Zhang, H. J. Phys. Chem. C 2015, 119, 6181-6194.

21. Furube, A.; Du, L.; Hara, K.; Katoh, R.; Tachiya, M. J. Am. Chem. Soc. 2007, 129, 1485214853.

22. Clavero, C. Nat. Photonics 2014, 8, 95-103.

23. Wu, K.; Rodríguez-Córdoba, W. E.; Yang, Y.; Lian, T. Nano Lett. 2013, 13, 5255-5263.

24. Mubeen, S.; Lee, J.; Singh, N.; Krämer, S.; Stucky, G. D.; Moskovits, M. Nat. Nanotechnol. 2013, 8, 247-251.

25. Zhong, Y.; Ueno, K.; Mori, Y.; Shi, X.; Oshikiri, T.; Murakoshi, K.; Inoue, H.; Misawa, H. Angew. Chem. Int. Ed. 2014, 53, 10350-10354.

26. Ueno, K.; Misawa, H. NPG Asia Mater. 2013, 5, e61.

27. Li, J.; Cushing, S. K.; Zheng, P.; Senty, T.; Meng, F.; Bristow, A. D.; Manivannan, A.; Wu, N. J. Am. Chem. Soc. 2014, 136, 8438-8449.

28. Knight, M. W.; Sobhani, H.; Nordlander, P.; Halas, N. J. Science 2011, 332, 702-704.

29. Zheng, B. Y.; Zhao, H.; Manjavacas, A.; McClain, M.; Nordlander, P.; Halas, N. J. Nat. Commun. 2015, 6, 7797.

30. Leenheer, A. J.; Narang, P.; Lewis, N. S.; Atwater, H. A. J. Appl. Phys. 2014, 115 (13), 134301.

31. Osterloh, F. E. Chem. Soc. Rev., 2013, 42, 2294-2320.

32. Osterloh, F. E.; Parkinson. B. A. MRS Bulletin, 2011, 36, 17-22.

33. Wu, N. Q.; Wang, J.; Tafen, D. N.; Wang, H.; Zheng, J.; Lewis, J. P.; Liu, X.; Leonard, S. S.; Manivannan, A. J. Am. Chem. Soc. 2010, 132, 6679-6685.

34. Wang, J.; Tafen, D. N.; Lewis, J. P.; Hong, Z.; Manivannan, A.; Zhi, M.; Li, M.; Wu, N. Q. J. Am. Chem. Soc. 2009, 131, 12290-12297.

35. Meng, F.; Hong, Z.; Arndt, J.; Li, M.; Zhi, M.; Yang, F.; Wu, N. Q. Nano Res. 2012, 5, 213221.

36. Yang, C.; Wang, Z.; Lin, T.; Yin, H.; Lv, X.; Wan, D.; Xu, T.; Zheng, C.; Lin, J.; Huang, F.; Xie, X.; Jiang, M. J. Am. Chem. Soc. 2013, 135, 17831-17838.

37. Asahi, R.; Morikawa, T.; Ohwaki, T.; Aoki, K.; Taga, Y. Science 2001, 293, 269-271.

38. Sakthivel, S.; Kisch, H. Angew. Chem., Int. Ed. 2003, 42, 4908-4911.

39. Chen, H.; Chen, C. K.; Chang, Y.; Tsai, C.; Liu, R.; Hu, S.; Chang, W.; Chen, K. Angew. Chem. Int. Ed. 2010, 49, 5966-5969.

40. Wang, H.; Wang, G.; Ling, Y.; Lepert, M.; Wang, C.; Zhang, J.; Li, Y. Nanoscale 2012, 4, 1463-1466.

41. Wang, G.; Yang, X.; Qian, F.; Zhang, J.; Li, Y. Nano Lett, 2010, 10, 1088-1092. Hensel, J.; Wang, G.; Li, Y.; Zhang, J. Nano Lett, 2010, 10, 478-483.

42. Buhbut, S.; Itzhakov, S.; Hod, I.; Oron, D.; Zaban, A. Nano Lett. 2013, 13, 4456-4461.

43. Kamat, P. V.; Tvrdy, K.; Baker, D. R.; Radich. J. G. Chem. Rev. 2010, 110, 6664-6688.

44. Kamat, P. V. J. Phys. Chem. C 2007, 111, 2834-2860. 
45. Berea, E. M.; Shalom, M.; Gimenez, S.; Hod, I.; Mora-Sero, I.; Zaban, A.; Bisquert, J. J. Am. Chem. Soc. 2010, 132, 6834-6839.

46. Gonzalez-Pedro, V.; Xu, X.; Mora-Sero, I.; Bisquert. J. ACS Nano, 2010, 4, 5783-5790.

47. Chakrapani, V.; Baker, D.; Kamat. P. V. J. Am. Chem. Soc. 2011, 133, 9607-9615.

48. Hyun, B.; Zhong, Y.; Bartnik, A. C.; Sun, L.; Abruña, H. D.; Wise, F. W.; Goodreau, J. D.; Matthews, J. R.; Leslie, T. M.; Borrelli, N. F. ACS Nano, 2008, 2, 2206-2212.

49. Cushing, S. K.; Wu, N. Q. Interface 2013, 22, 63-67.

50. Tian, Y.; Tatsuma, T. J. Am. Chem. Soc. 2005, 127, 7632-7637. Tian, Y.; Tatsuma, T. Chem. Commun. 2004, 1810-1811.

51. Cushing, S. K.; Li, J.; Meng, F.; Senty, T. R.; Suri, S.; Zhi, M.; Li, M.; Brristow, A. D.; Wu, N. Q. J. Am. Chem. Soc. 2012, 134, 15033-15041.

52. Li, J.; Cushing, S.; Bright, J.; Meng, F.; Sentry, T. R.; Zheng, P.; Bristow, A. D.; Wu, N. Q. ACS Catalysis. 2013, 3, 47-51.

53. Li, J.; Cushing, S. K.; Zheng, P.; Meng, F.; Chu, D.; Wu. N. Q. Nat. Commun. 2013, 4, 2651.

54. Tada, H.; Mitsui, T.; Kiyonaga, T.; Akita, T.; Tanaka, K. Nature Mater. 2006, 5, 782-786.

55. Yun, H. J.; Lee, H.; Kim, N. D.; Lee, D. M.; Yu, S.; Yi, J. ACS Nano 2011, 5, 4084-4090.

56. Fang, J.; Zhang, Z.; Yuan, Y.; Cao, S.; Wang, Z.; Yin, L.; Xiao, Y.; Xue, C. ACS Appl. Mater. Interfaces, 2013, 5, 8088-8092. Yu, Z.; Xie, Y.; Liu, G.; Lu, G.; Ma, X.; Chen. H. J. Mater. Chem. A 2013, 1, 2773-2776.

57. Lv, T.; Pan, L.; Liu, X.; Sun. Z. Electrochimica Acta, 2012, 83, 216-220.

58. Zhu, H.; Yang, B.; Xu, J.; Fu, Z.; Wen, M.; Guo, T.; Fu, S.; Zuo, J.; Zhang, S. Appl. Catal. B Environmental, 2009, 90, 463-469.

59. Cánovas, E.; Moll, P.; Jensen, S. A.; Gao, Y.; Houtepen, A. J.; Siebbeles, L. D. A.; Kinge, S.; Bonn, M. Nano Lett. 2011, 11, 5234-5239.

60. Tisdale, W. A.; Williams, K. J.; Timp, B. A.; Norris, D. J.; Aydil, E. S.; Zhu, X.Y. Science 2010, 328, 1543-1547.

61. Shen, Q.; Ayuzawa, Y.; Katayama, K.; Sawada, T.; Toyoda, T. Appl. Phys. Lett. 2010, 97, 263113.

62. Blackburn, J. L.; Selmarten, D. C.; Nozik, A. J. J. Phys. Chem. B 2003, 107, 14154-14157.

63. Blackburn, J. L.; Selmarten, D. C.; Ellingson, R. J.; Jones, M.; Micic, O.; Nozik, A. J. J. Phys. Chem. B 2005, 109, 2625-2631.

64. Tvrdy, K.; Frantsuzov, P. A.; Kamat, P. V. Proc. Natl. Acad. Sci. U. S. A. 2011, 108, 29-34.

65. Jin, S.; Lian, T. Nano Lett. 2009, 9, 2448-2454.

66. Pernik, D.; Tvrdy, K. J. Phys. Chem. C 2011, 115, 13511-13519.

67. Gao, Y.; Suchand Sandeep, C. S.; Schins, J. M.; Houtepen, A. J.; Siebbeles, L. D. A. Nat. Commun. 2013, 4, 2329.

68. Zhu, H.; Yang, Y.; Hyeon-Deuk, K.; Califano, M.; Song, N.; Wang, Y.; Zhang, W.; Prezhdo, O. V.; Lian, T. Nano Lett. 2014, 14, 1263-1269.

69. Liu, B.; Aydil, E. S. J. Am. Chem. Soc., 2009, 131, 3985-3990.

70. Li, J.; Hoffmann, M. W. G.; Shen, H.; Fabrega, C.; Prades, J. D.; Andreu, T.; HernandezRamirez, F.; Mathur, S. J. Mater. Chem., 2012, 22, 20472-20476.

71. Linnros, J. J. Appl. Phys. 1998, 84, 275.

72. Linnros, J. J. Appl. Phys. 1998, 84, 284.

73. Grivickas, V.; Linnros, J. In Charecterization of Materials; John Wiley and Sons, Inc., 2012; 
p. 658.

74. Sakata, T.; Hashimoto, K.; Hiramoto, M. J. Phys. Chem. 1990, 94, 3040-3045.

75. Stockwell, D.; Yang, Y.; Huang, J.; Anfuso, C.; Huag, Z.; Lian, T. J. Phys. Chem. C 2010, 114, 6560-6566.

76. Bisquert, J.; Zaban, A.; Salvador, P. J. Phys. Chem. B 2002, 106, 8774-8782.

77. Hirakawa, T.; Kamat, P. Langmuir 2004, 20, 5645-5647.

78. Pu, Y.; Wang, G.; Chang, K.; Ling, Y.; Lin, Y.; Fitzmorris, B. C.; Liu, C.; Lu, X.; Tong, Y.; Zhang, J. Z.; Hsu, Y.; Li, Y. Nano Lett., 2013, 13, 3817-3823.

79. Jakob, M.; Levanon, H.; Kamat. P. Nano Lett. 2003, 3, 353-358.

80. Subramanian, V.; Wolf, E. E.; Kamat. P. J. Am. Chem. Soc. 2004, 126, 4943-4950.

81. D. Bahnemann, J. Phys. Chem. B 1997, 5647, 4265-4275.

82. Katoh, R.; Murai, M.; Furube, A. Chem. Phys. Lett. 2010, 500, 309-312.

83. Tamaki, Y.; Hara, K.; Katoh, R. J. Phys. Chem. C 2009, 113, 11741-11746.

84. Tamaki, Y.; Furube, A.; Murai, M.; Hara, K.; Katoh, R.; Tachiya, M. Phys. Chem. Chem. Phys. 2007, 9, 1453-1460.

85. Yoshihara, T.; Katoh, R.; Furube, A. J. Phys. Chem. B 2004, 3817-3823.

86. Froitzheim, A.; Stangl, R.; Elstner, L.; Kriegel, M.; Fuhs, W. In Photovoltaic Energy Conversion, 2003. Proceedings of 3rd World Conference on; 2003; pp. 279-282.

87. Markus, T.; Itzhakov, S. J. Phys. Chem. C 2011, 115, 13236-13241.

88. Khon, E.; Mereshchenko, A.; Tarnovsky, A. N.; Acharya, K.; Klinkova, A.; Hewa-Kasakarage, N. N.; Nemitz, I.; Zamkov, M. Nano Lett. 2011, 11, 1792-1799.

89. Kobayashi, Y.; Nonoguchi, Y.; Wang, L.; Kawai, T.; Tamai, N. J. Phys. Chem. Lett. 2012, 3, 1111-1116.

90. Dreaden, E. C.; Neretina, S.; Qian, W.; El-Sayed, M. A.; Hughes, R. A.; Preston, J. S.; Mascher, P. J. Phys. Chem. C 2011, 115, 5578-5583.

91. Zhang, W.; Govorov, A.; Bryant, G. Phys. Rev. Lett. 2006, 97, 146804.

92. Artuso, R. D.; Bryant, G. W. Phys. Rev. B 2010, 82, 195419.

93. Mongin, D.; Shaviv, E.; Maioli, P.; Crut, A.; Banin, U.; Del Fatti, N.; Vallée, F. ACS Nano 2012, 6, 7034-7043.

94. Wu, K.; Rodríguez-Córdoba, W. E.; Yang, Y.; Lian, T. Nano Lett. 2013, 13, 5255-5263. 


\section{Chapter 4. Near Field Enhancement}

\subsection{Introduction}

As introduced in Chapter 1, the hallmark of plasmonics is the local field enhancement. The plasmon takes the energy of incident light and converts it into sub-free space wavelength collective electron oscillations. In this process, energy is effectively focused into a smaller volume, allowing its amplification in the local electromagnetic field created by the collective charge oscillations against the ionic core of the metal. By designing nanoshapes with sharp edges, the field amplification can range from 10 to $10^{4}$ or higher that of incident light, acting similar in concept to a lightning rod. ${ }^{1}$ As shown in Chapter 2, this can allow for a large increase in absorption in a semiconductor if coupling can occur between the two systems. However, the local field is not just useful for light trapping, it can actually extend the light absorption spectral range if the local field amplification is large enough to populate near-band edge states, acting similar in effect to the hot carrier transfer mechanism discussed in Chapter 3.

Originally, confusion existed about the role of the plasmon's local field in enhancing solar energy conversion. ${ }^{2-10}$ It was debated that large enhancements in photocatalysis, especially those near the semiconductor band edge that followed the plasmon's absorption, were originating in hot electron transfer. However, the same enhancements still existed when an insulating spacer layer was placed between metal and semiconductor, preventing charge transfer. ${ }^{7}$ Even more intriguing, the enhancement was actually found larger when the metal and semiconductor were not in contact. ${ }^{10}$ If the plasmon and semiconductor were coupling through non-radiative, near-field effects, the question of by what mechanism had to be answered. Dipole-dipole coupling with metal nanoparticles was known to quench semiconductor fluorescence by Förster Resonance Energy Transfer (FRET) ${ }^{11-14}$ with near 100\% efficiency, but if the direction of the energy transfer could change direction remained unknown, and if so what would be the upper limit of the process.

In the following sections these questions are addressed, isolating and describing the mechanism of near field coupling between plasmon and semiconductor, as well as exploring its efficiency.

\subsection{Isolating the Near-Field Plasmonic Enhancement Mechanism}

Plasmonics improves the solar-energy-conversion efficiency by (i) increasing light absorption by scattering (see Chapter 2), (ii) extending light absorption by hot carrier transfer (see Chapter 3), and (iii) potentially exciting electron-hole pairs in the semiconductor by transferring the plasmonic energy from the metal to the semiconductor. So far, the plasmon-induced charge separation mechanism of case (iii) remains unclear. Hot carrier transfer (also known as direct electron transfer (DET)) occurs from the plasmonic metal to the conduction band of the semiconductor when they are in direct contact (Figure 4.1c). ${ }^{15-20}$ DET depends on the alignment of the band levels of the semiconductor and Fermi level of the plasmonic metal, so it is possible for electrons or holes to be transferred from the metal into the semiconductor at energies below the band gap if the electronic energy levels match. DET occurs after the excitation and subsequent decoherence of the plasmon, which leaves a population of hot electrons that are able to undergo transfer to the semiconductor. For example, plasmon-mediated hot electrons have been confirmed to be injected from gold nanoparticles to the conduction band of $\mathrm{TiO}_{2} \cdot{ }^{17}$

However, DET is not the only proposed mechanism. Recent studies have found that the photocatalysis of $\mathrm{TiO}_{2}$ is still enhanced after an insulating interlayer is added between the metal and the semiconductor to prevent DET. It was proposed that the plasmon-mediated electromagnetic field (EMF) radiatively contributed to the local generation of electron-hole pairs in the semiconductor (Figure 4.1d). ${ }^{3-10}$ The EMF 
induced charge separation mechanism can only create carriers for energies above the band gap of the semiconductor. In the present work, it is proposed that the electromagnetic field mediated plasmonic energy transfer can take the form of a resonant energy transfer (RET) process (Figure 4.1e). The RET process is proposed to be an alternative, nonradiative mechanism of SPR-induced charge separation in semiconductors. Whereas the radiative EMF mechanism increases the rate of interband transitions in the semiconductor due to the increased EM field, the RET process directly excites electron-hole pairs in the semiconductor nonradiatively through the relaxation of the localized surface plasmon dipole. Plasmoninduced RET in the near field is similar to Förster resonance energy transfer (FRET), where the LSPR dipole replaces the fluorescent system.
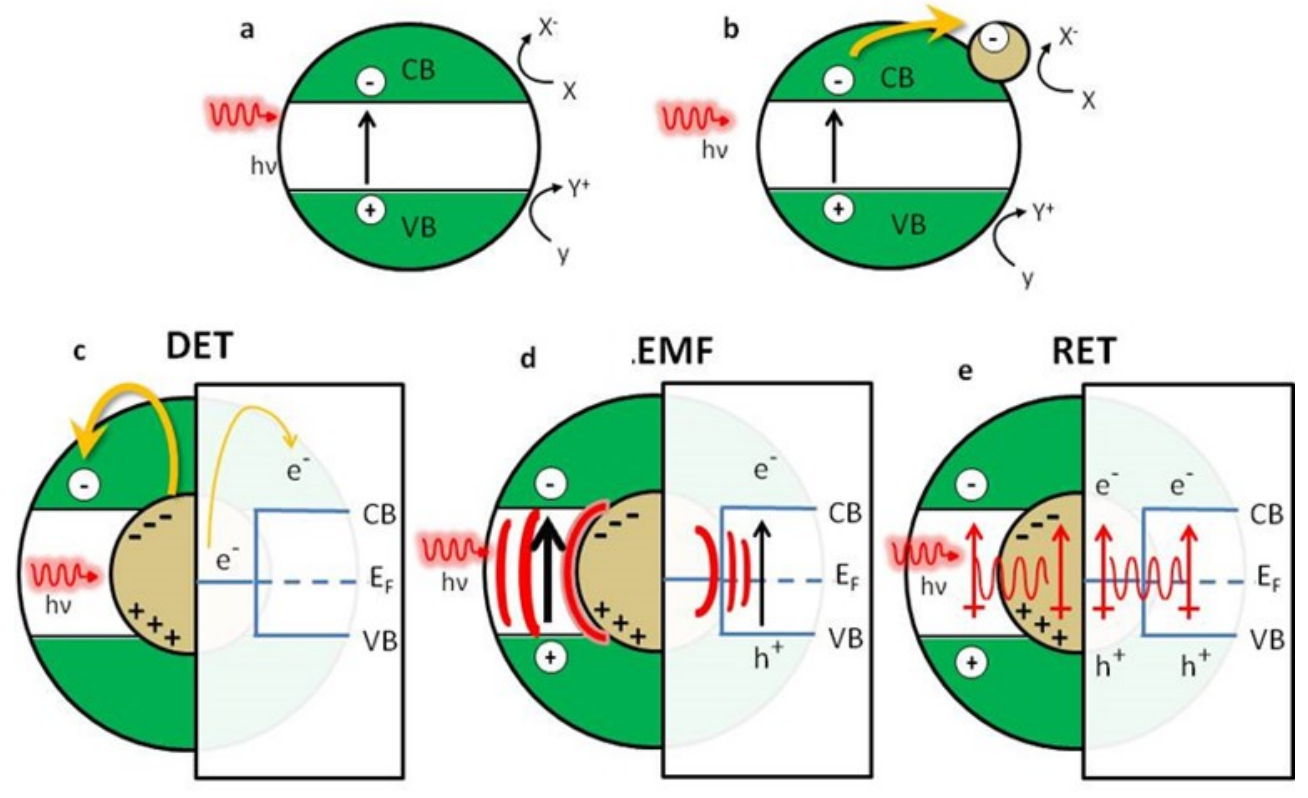

Figure 4.1. Charge separation mechanisms in various photocatalytic nanostructures. a, Photoexcited (hv) semiconductors produce electrons (holes) in the conduction (valence) band [CB (VB)], each contributing to chemical reactions $\left(X+e^{-}=X^{-}\right)$and $\left(Y+h^{+}=Y^{+}\right)$at their surface. b, Metal nanoparticles can act as co-catalysts to provide additional surface sites via the trapping of electrons. Metal@semiconductor structures can increase charge separation by c, direct electron transfer (DET) of hot electrons contained in LSPR to the semiconductor, d, electromagnetic field enhancement (EMF) of the semiconductor charge separation process, and e, resonant energy transfer (RET) from the LSPR dipole to the electron hole pair in the semiconductor shell.

If RET can efficiently create electron-hole pairs in the semiconductor it will broadly increase the scope of materials which can be enhanced by plasmonic energy transfer. DET can transfer charge carriers to the semiconductor at energies below band gap, however DET material selection is limited by the electronic band structure alignment of the semiconductor and metal. In order for the plasmonic electrons to transfer into the conduction band of the semiconductor charge equilibrium must be maintained, often necessitating the use of hole scavengers. The EMF mechanism does not suffer from charge equilibration issues since the interband transition rate in the semiconductor is increased radiatively by the local field. However the materials selection is still limited since EMF cannot enhance charge separation at energies below the band gap. EMF can only enhance the rate of the semiconductors interband transitions, not extend carrier creation to longer wavelengths. Like EMF, the nonradiative dipole-dipole energy transfer of RET is not limited by electronic band structure matching and charge equilibration, since the energy of the plasmon transfers to an electron-hole pair in the semiconductor through a near field electromagnetic interaction. However, unlike 
previous reports of EMF, it is demonstrated in this paper that RET can create electron-hole pairs in the semiconductor at energies both above and below the band gap due to nonradiative coupling with optically inaccessible and optically inefficient states at the band edge. RET can overcome the charge equilibration issues of DET while still enhancing carrier creation at energies below the band gap, unlike EMF.

Previous studies on determining the plasmon-enhanced photocatalysis mechanism have placed metal nanoparticles on the surface of semiconductors as a co-catalyst. The observed enhancement may be due to both plasmonic enhancement of the catalyst and/or simply self-catalysis of the co-catalyst. Other experiments have randomly dispersed metal nanoparticles into a semiconductor matrix, leading to a large variation in the SPR position and local electromagnetic field distribution, which affect the transfer mechanism. Unambiguously resolving the dominant energy transfer mechanism requires the design of controllable metal/semiconductor composite nanostructures.

To investigate the possibility of plasmonic energy transfer by RET in this Section, ${ }^{21}$ core-shell $\mathrm{Au} @ \mathrm{Cu}_{2} \mathrm{O}$ (Figure 4.2c) and sandwiched $\mathrm{Au@SiO} \mathrm{S}_{2} @ \mathrm{Cu}_{2} \mathrm{O}$ nanoparticles (Figure 4.2d) have been synthesized. These structures have been engineered to isolate the plasmonic energy transfer mechanisms as follows. Gold and $\mathrm{Cu}_{2} \mathrm{O}$ are chosen due to the overlap between the localized surface plasmon resonance (LSPR) of the Au core and the band gap absorption of $\mathrm{Cu}_{2} \mathrm{O}$ which allows for RET. Embedding the metal inside of the semiconductor eliminates possible self-catalysis effects of the metal (Fig. 4.1b). The thin $\mathrm{SiO}_{2}$ layer acts to electronically insulate the metal from the semiconductor while still allowing unattenuated propagation of the optical field around the nanoparticle. The interlayer effectively blocks direct electron transfer (DET) between the $\mathrm{Au}$ and $\mathrm{Cu}_{2} \mathrm{O}$ while still allowing for RET. In addition, significant light scattering and enhancements to the optical path length are only seen in large plasmonic metal nanoparticles $(>50 \mathrm{~nm}) .{ }^{22}$ The sandwich structure designed for this study utilizes a gold core that is only $20 \mathrm{~nm}$ in a diameter, eliminating enhancements due purely to increased scattering (Process ii). The designed sandwich structure thus allows us to investigate how the plasmonic energy of the Au core is transferred to the semiconductor shell in the presence and absence of an insulating interlayer without the effects of light scattering and metal catalysis.

This Section ${ }^{21}$ uses a controlled structure, designed to isolate the electromagnetic field mediated plasmonic energy transfer mechanisms from the DET mechanism so that it can be determined if a RET process is responsible for the enhanced creation of carriers in the semiconductor. A combination of photocatalysis action spectrum, transient-absorption spectroscopy, and theoretical calculation show for the first time that electron-hole pairs in the semiconductor are created via resonant energy transfer (RET) from the plasmonic metal (Figure 4.1e), which in the near field is similar to Förster resonance energy transfer (FRET). The RET process excites electron-hole pairs in the semiconductor through the relaxation of the localized surface plasmon dipole. The radiative coupling component originally proposed in EMF is not seen in our experiments, rather the electromagnetic field interaction is dominated in the near field by the nonradiative RET. The RET process is clearly distinguished from DET.

\subsubsection{Methods}

Synthesis of Au nanospheres and $\mathrm{Au} @ \mathrm{SiO}_{2}$ nanospheres: Au nanospheres were synthesized by reducing $\mathrm{HAuCl}_{4}$ with sodium citrate according to the Frens' method. ${ }^{23} 4 \mathrm{~mL}$ of $1 \mathrm{wt} \%$ sodium citrate was added to $200 \mathrm{~mL}$ of $5 \mathrm{mM} \mathrm{HAuCl}_{4}$ solution at a boil. Silica was coated on the Au nanospheres using the sodium silicate hydrolyzing technique in basic solution. ${ }^{24} 200 \mathrm{uL}$ of $2 \mathrm{mM}$ aminopropyltrimethoxysilane (APS) was added into $20 \mathrm{~mL}$ of the Au colloids and stirred for $30 \mathrm{~min}$ followed by $500 \mathrm{uL}$ of $0.54 \mathrm{wt} \%$ sodium silicate and $30 \mathrm{~min}$ stirring. Finally $20 \mathrm{~mL}$ of ethanol was added and the mixture stood for 2 days. 
Synthesis of $\mathrm{Au} @ \mathrm{Cu}_{2} \mathrm{O}$ core-shell nanoparticles and Au@SiO $2 @ \mathrm{Cu}_{2} \mathrm{O}$ sandwich nanoparticles: The $\mathrm{Au} @ \mathrm{Cu}_{2} \mathrm{O}$ core-shell nanoparticles were synthesized following a previously reported procedure. $2 \mathrm{~mL}$ of the as-prepared Au colloids was added to the solution containing $0.1 \mathrm{M} \mathrm{CuCl}_{2}$ and $0.0338 \mathrm{M}$ sodium dodecyl sulfate (SDS). Next $0.25 \mathrm{~mL}$ of $1 \mathrm{M} \mathrm{NaOH}$ and $0.45 \mathrm{~mL}$ of $0.2 \mathrm{M} \mathrm{NH}_{4} \mathrm{OH} \cdot \mathrm{HCl}$ were added with stirring. The synthesis procedure for the $\mathrm{Au} @ \mathrm{SiO}_{2} @ \mathrm{Cu}_{2} \mathrm{O}$ sandwich nanoparticles was the same as the $\mathrm{Au} @ \mathrm{Cu}_{2} \mathrm{O}$ except that the Au colloids were replaced with $\mathrm{Au} @ \mathrm{SiO}_{2}$ colloids.

Characterization: The nanoparticles were observed using a JEOL 7600F field emission scanning electron microscope. X-ray photoelectron spectroscopy was performed using a PHI 5000 Versa Probe. Exctinction was measured using a Shimadzu 2550 spectrometer.

Measurement of photocatalytic activity under visible and monochromatic light irradiation: The photocatalytic activity was evaluated by the degradation of methyl orange. A photoreactor equipped with fourteen $8 \mathrm{~W}$ visible light lamps (Cool white Florescent, range from 400-700 nm) was used as the light source. $0.15 \mathrm{mg} / \mathrm{mL}$ photocatalysts were dispersed in $40 \mathrm{~mL}$ of $50 \mathrm{mg} / \mathrm{L}$ methyl orange and allowed to reach adsorption/desorption equilibrium. The residual amount of $\mathrm{MO}$ at various times was determined using a Shimadzu spectrophotometer. The same procedure was followed to for the wavelength dependent photocatalytic activity except the light source was a $300 \mathrm{~W}$ Xe lamp coupled to a monochromator.

Transient Absorption: Non-degenerate pump-probe experiment were performed with 100-fs pulses from a $1 \mathrm{KHz} \mathrm{Ti:sapphire} \mathrm{laser} \mathrm{amplifier} \mathrm{(Libra)} \mathrm{and} \mathrm{an} \mathrm{optical} \mathrm{parametric} \mathrm{amplifier} \mathrm{(OPerA} \mathrm{Solo).} \mathrm{Pump} \mathrm{pulses}$ from the OPerA Solo where doubled in a $\beta$-barium borate crystal to provide a tuneable wavelength source from $620 \mathrm{~nm}$ to $750 \mathrm{~nm}$. Probe pulses were taken directly from the Libra. Time-delay between the pulses was controlled by a motion control system with a 4-ns time range. Focused beam spot sizes where $400 \mu \mathrm{m}$ for the pump and $240 \mu \mathrm{m}$ for the probe. Samples were dispersed in a $\mathrm{KBr}$ matrix. Transmission probe radiation was collected on a photo-detector and recorded with a lock-in amplifier.

Discrete Dipole Approximation Simulations: Simulations of the local electromagnetic field were performed using the free program DDSCAT. ${ }^{25}$ The refractive index for $\mathrm{Cu}_{2} \mathrm{O}$ and $\mathrm{Au}$ was taken from Palik ${ }^{26}$ and the online Sopra $\mathrm{N}$ and $\mathrm{K}$ database. The input source was $656 \mathrm{~nm}$ and linearly polarized along the $\mathrm{X}$ axis.

\subsubsection{Results}

Structures of Photocatalysts. The high-resolution transmission electron micrographs (HRTEM) in Figure 4.2 show the result of the synthesis of the core-shell and sandwich nanostructures. First 20-nm diameter $\mathrm{Au}$ nanospheres were synthesized (Figure 4.2a), which were then coated with a $\sim 5 \mathrm{~nm}$ thick $\mathrm{SiO}_{2}$ layer to form a $\mathrm{Au} @ \mathrm{SiO}_{2}$ core-shell structure (Figure 4.2b), and a $\sim 25 \mathrm{~nm}$ thick $\mathrm{Cu}_{2} \mathrm{O}$ layer to form a $\mathrm{Au} @ \mathrm{Cu}_{2} \mathrm{O}$ core-shell nanoparticles (Figure 4.2c). The former core-shells where coated with a $\sim 25 \mathrm{~nm}$ thick $\mathrm{Cu}_{2} \mathrm{O}$ layer to create a $\mathrm{Au} @ \mathrm{SiO}_{2} @ \mathrm{Cu}_{2} \mathrm{O}$ structure (Figure 4.2(d, g)). From the HRTEMs, the (111) planes of the single-crystalline Au cores were visible and $\mathrm{SiO}_{2}$ was observed to be amorphous. The $\mathrm{Cu}_{2} \mathrm{O}$ shells were polycrystalline, regardless of being coated directly on the Au core or the $\mathrm{SiO}_{2}$ layer. The chemical composition of the Au cores and the $\mathrm{Cu}_{2} \mathrm{O}$ shells was confirmed by X-ray photoelectron spectroscopy and $\mathrm{Cu}_{2} \mathrm{O}$ particles were also synthesized for comparison of optical and photochemical properties as detailed in-depth in Reference 21. 

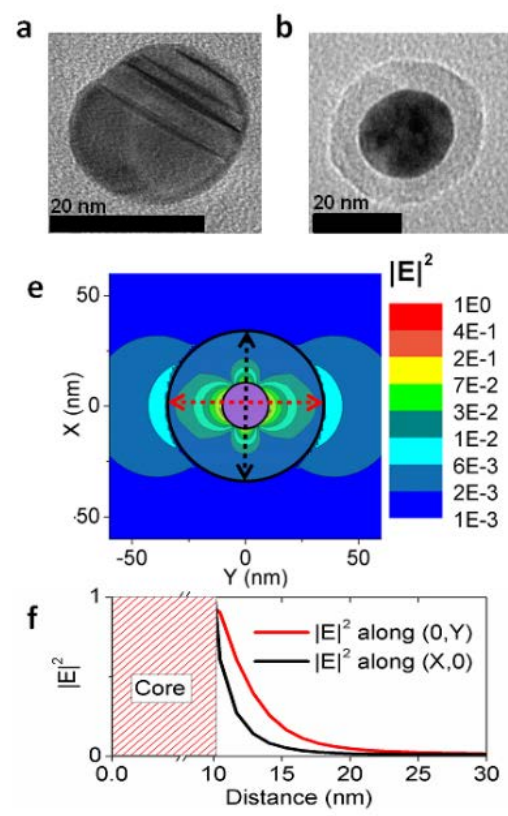

c
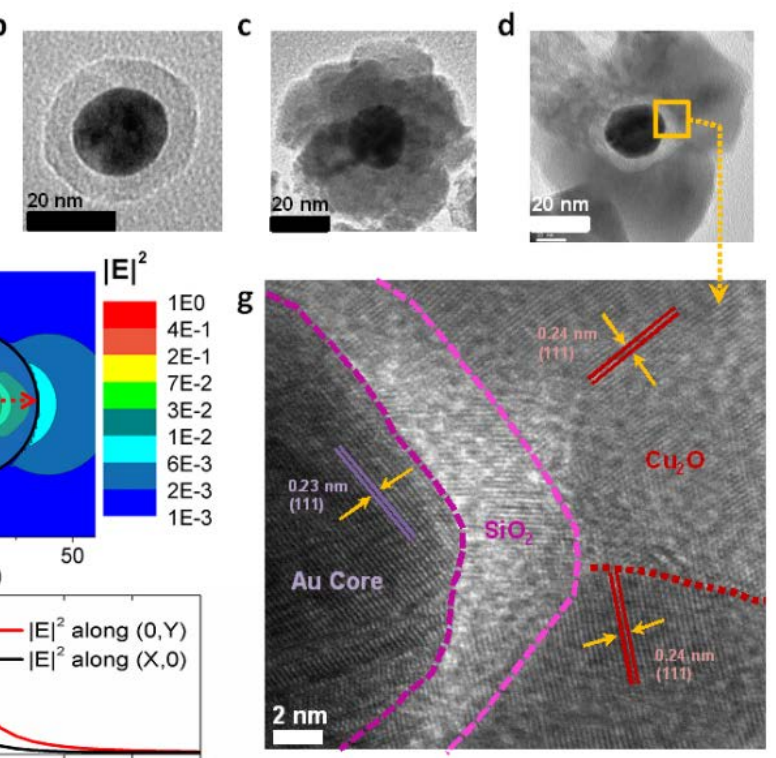

Figure 4.2. SEM images of the core-shell and sandwich nanoparticles. High-resolution transmission electron micrographs of a, 20-nm uncoated Au nanosphere, b, $\mathrm{Au} @ \mathrm{SiO}_{2}$ core-shell structure, c, $\mathrm{Au} @ \mathrm{Cu}_{2} \mathrm{O}$ core-shell structure, and d,Au@SiO${ }_{2} @ \mathrm{Cu}_{2} \mathrm{O}$ sandwich structure. g, An enlargement of the micrograph for the interface regions of the sandwich structure shows the various crystal orientations. e, Discrete dipole approximation simulation of the local electromagnetic field created by the plasmonic core and extending into the surrounding $\mathrm{Cu}_{2} \mathrm{O}$ shell for input radiation along the $\mathrm{X}$ axis, with $\mathbf{f}$, cross-sectional slices showing the EM field as a function of distance from the center of the Au core

Light Absorption and Photocatalytic Activity. Figure 4.3 shows ultraviolet-visible extinction spectra for the $\mathrm{Cu}_{2} \mathrm{O}, \mathrm{Au} @ \mathrm{Cu}_{2} \mathrm{O}$ and $\mathrm{Au} @ \mathrm{SiO}_{2} @ \mathrm{Cu}_{2} \mathrm{O}$ nanoparticles dispersed in the aqueous solutions. Bare gold nanospheres exhibited a broad LSPR centered at $520 \mathrm{~nm}$. Coating the Au nanospheres with the $\mathrm{SiO}_{2}$ layer resulted in a red-shift of the plasmon peak to $525 \mathrm{~nm}$. Pure $\mathrm{Cu}_{2} \mathrm{O}$ samples showed interband absorption,

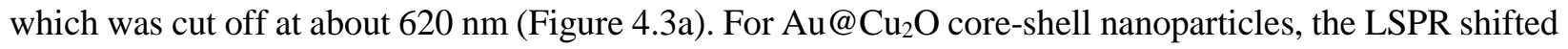
to $650 \mathrm{~nm}$ (Figure 4.3b) due to a decrease in the contrast of the effective dielectric constant between the core and cladding. Similarly, the $\mathrm{Au} @ \mathrm{SiO}_{2} @ \mathrm{Cu}_{2} \mathrm{O}$ sandwich structure showed a shifted LSPR at $600 \mathrm{~nm}$ (Figure 4.3c) due to a change in the dielectric contrast. At wavelengths below $500 \mathrm{~nm}$, increased scattering increased the extinction for both the core-shell and sandwich nanoparticles. The local electromagnetic field associated with the plasmon resonance was determined by discrete dipole approximation simulations assuming perfect spherical symmetry (Figure 4.2(e,f)). The local nature of the LSPR field suggests that if plasmon-mediated charge separation exists in the semiconductor it will proceed through RET. Quadrupole moment terms are weak and can be neglected.

The photocatalytic activity of the $\mathrm{Au} @ \mathrm{SiO}_{2} @ \mathrm{Cu}_{2} \mathrm{O}, \mathrm{Au} @ \mathrm{Cu}_{2} \mathrm{O}$ and pure $\mathrm{Cu}_{2} \mathrm{O}$ nanoparticles were evaluated by the photo-degradation rate of methyl orange in the aqueous solution under visible-light irradiation (Figure 4.3d). The corresponding kinetic data is shown in Reference 21. The $\mathrm{Au@} \mathrm{Cu}_{2} \mathrm{O}$ coreshell nanoparticle showed better photocatalytic activity than the pure $\mathrm{Cu}_{2} \mathrm{O}$ nanoparticles (Figure 4.3d). The Au core was isolated from the reactant by the semiconductor shell, ruling out the chemical catalysis effect of the Au nanoparticles. The $\mathrm{Au} @ \mathrm{SiO}_{2} @ \mathrm{Cu}_{2} \mathrm{O}$ sandwich nanoparticles exhibited the best photocatalytic activity among the three samples tested. It has been reported that LSPR-induced localized heating in the metal nanoparticles can lead to thermo-chemical degradation of the organic compunds. ${ }^{27}$ To ensure the 
enhanced photocatalytic activity was not due to heating, the degradation of methyl orange was also measured under visible-light irradiation in the presence of the bare $\mathrm{Au}$ nanospheres and the $\mathrm{Au} @ \mathrm{SiO}_{2}$ nanoparticles. No photocatalytic activity was measured, showing that neither the bare Au nanospheres nor the Au@SiO 2 nanoparticles were able to thermally activate the decomposition of methyl orange under visible-light irradiation.
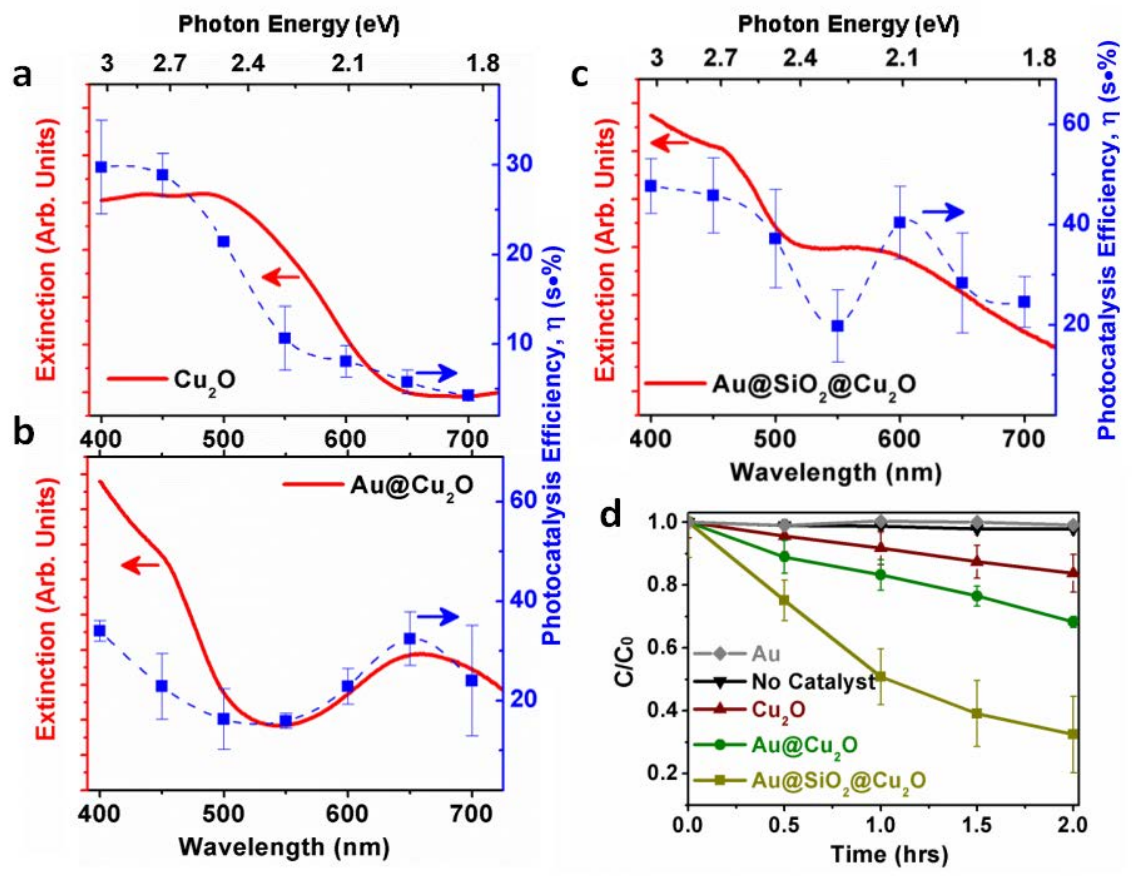

Figure 4.3. Ultraviolet-visible spectra and photocatalytic action spectra for $\mathbf{a}, \mathrm{Cu}_{2} \mathrm{O}, \mathbf{b}, \mathrm{Au} @ \mathrm{Cu}_{2} \mathrm{O}$ and $\mathbf{c}$, $\mathrm{Au} @ \mathrm{SiO}_{2} @ \mathrm{Cu}_{2} \mathrm{O}$; d, Visible-light photo-degradation of methyl orange versus time is monitored for no catalyst, uncoated $\mathrm{Au}, \mathrm{Cu}_{2} \mathrm{O}$ control, $\mathrm{Au} @ \mathrm{Cu}_{2} \mathrm{O}, \mathrm{Au} @ \mathrm{SiO}_{2} @ \mathrm{Cu}_{2} \mathrm{O}$ nanostructures.

To determine whether the LSPR was responsible for enhancement of the photocatalytic activity, the extinction spectra of the nanoparticles were directly compared to the action spectra of apparent photocatalysis efficiency (Figure 4.3(a-c)). The apparent photocatalysis efficiency per unit time is defined

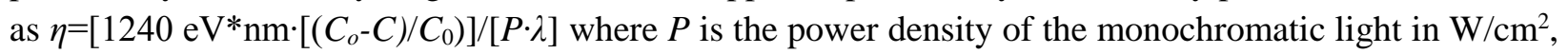
and $\lambda$ is the wavelength in $\mathrm{nm} . \mathrm{C}_{0}$ and $C$ are the initial and final concentrations of reactant in mol/L after a certain irradiation time, respectively. The apparent photocatalysis efficiency was defined to normalize the photocatalysis by the differing power of monochromated light at each wavelength used in the action spectrum. It was not intended to reflect an absolute quantum yield for photocatalysis. The action spectra shows the apparent photocatalysis efficiency as a function of the wavelength of incident monochromatic light. The pure $\mathrm{Cu}_{2} \mathrm{O}$ nanoparticles exhibited photocatalytic activity that followed the extinction spectrum (Figure 4.3a), with negligible photocatalytic activity (efficiency) below the band edge. It should be noted that the photocatalysis was weak for excitation at $550 \mathrm{~nm}$ near the band edge of $\mathrm{Cu}_{2} \mathrm{O}$, which indicated that the absorption at the wavelength may be dominated by trap states which were inefficient for photocatalysis. The trend was consistent for the $\mathrm{Cu}_{2} \mathrm{O}, \mathrm{Au} @ \mathrm{Cu}_{2} \mathrm{O}$, and $\mathrm{Au} @ \mathrm{SiO}_{2} @ \mathrm{Cu}_{2} \mathrm{O}$ nanoparticles, independent of the structure or the possible transfer mechanisms.

The photocatalysis action spectrum (Figure 4.3b) for the $\mathrm{Au@} \mathrm{Cu}_{2} \mathrm{O}$ nanoparticles also followed the trend of the extinction spectrum, showing significant enhancement at the LSPR wavelengths as compared to pure $\mathrm{Cu}_{2} \mathrm{O}$. This enhancement can only happen if light excited the plasmon and then energy or charge 
was transferred to the semiconductor to drive the photocatalysis. While it is likely that RET mediates the energy transfer from plasmon to semiconductor because of the overlap of the LSPR and the $\mathrm{Cu}_{2} \mathrm{O}$ conduction band, the electromagnetic interaction mechanism cannot be separated from DET using the $\mathrm{Au} @ \mathrm{Cu}_{2} \mathrm{O}$ photocatalysis data alone. Only the involvement of the LSPR in creating charge separation can be proven from the $\mathrm{Au} @ \mathrm{Cu}_{2} \mathrm{O}$ nanoparticles.

The photocatalysis action spectrum of the $\mathrm{Au} @ \mathrm{SiO}_{2} @ \mathrm{Cu}_{2} \mathrm{O}$ sandwich nanoparticles was examined to determine whether the DET or RET mechanism was dominant. Once again the action spectrum generally followed the extinction spectrum (Figure 4.3c), with strong enhancement as compared to pure $\mathrm{Cu}_{2} \mathrm{O}$. DET was suppressed due to the insulating silica layer that acted as a barrier for electron transfer. The enhanced photocatalytic activity at the LSPR wavelengths must therefore be due to an interaction between the LSPR and semiconductor through the local electromagnetic field. RET is the most probable near-field interaction mechanism due to coupling of the large plasmonic dipole moment to the electron-hole pair dipole moment in the semiconductor shell. The relaxation of the LSPR dipole will lead to the excitation of electron-hole pairs in the semiconductor resulting in enhanced photocatalysis.

The relative strength of the energy transfer depends on the overlap integral of the plasmon resonance and the conduction band as shown by the transfer rate from FRET

$$
k_{\text {transfer }}=\frac{1}{\tau_{\text {donor }}}\left(\frac{R_{0}}{R}\right)^{6},
$$

where $\mathrm{R}_{0}$ (given in Angstroms) can be calculated as

$$
R_{0}=0.2108\left(\kappa^{2} \Phi_{0} n^{-4} J\right)^{1 / 6},
$$

where $\kappa$ is an orientation factor usually taken as $2 / 3, \mathrm{n}$ is the refractive index, $\Phi_{0}$ is the quantum yield of the donor, and $\mathrm{J}$ is the normalized overlap integral between the donor spectrum $\mathrm{F}_{\mathrm{D}}$ and the acceptor spectrum $\varepsilon_{\mathrm{A}}$

$$
J=\int F_{D}(\lambda) * \epsilon_{A}(\lambda) * \lambda^{4} d \lambda .
$$

Details of extracting the overlap integral are discussed later, but the overlaps are shown in Figures 4.4 for the core-shell and sandwich structures. Since the $\mathrm{Au@SiO} \mathrm{S}_{2} @ \mathrm{Cu}_{2} \mathrm{O}$ structure had the largest overall in spectral activity and the widest overlap integral, it should show the highest photocatalytic efficiency according to the RET theory. This initial comparison agrees with the theory of RET and is supported by the fast photo-degeneration observed in the $\mathrm{Au@SiO}{ }_{2} @ \mathrm{Cu}_{2} \mathrm{O}$ under white-light illumination (Figure 4.3d).
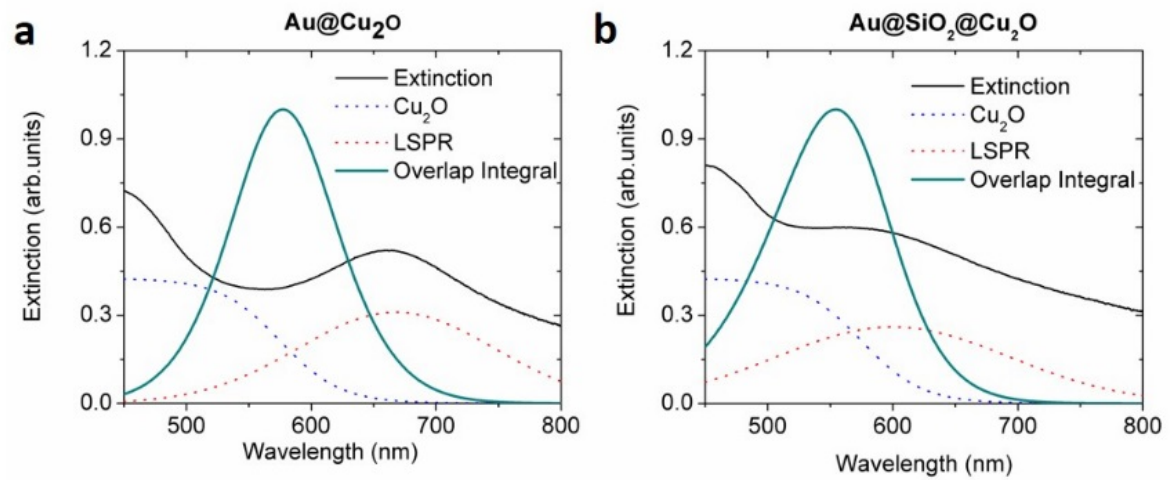

Figure 4.4. Overlap Function for $\mathrm{Cu}_{2} \mathrm{O}$ and plasmonic gold in $\mathbf{a}, \mathrm{Au} @ \mathrm{Cu}_{2} \mathrm{O}$ and $\mathbf{b}, \mathrm{Au} @ \mathrm{SiO}_{2} @ \mathrm{Cu}_{2} \mathrm{O}$. The solid black

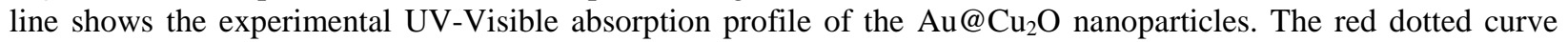
shows the fit of the SPR with a Gaussian function. The blue dotted line shows the fit of the $\mathrm{Cu}_{2} \mathrm{O}$ band gap by a Boltzmann distribution. The aqua solid line shows the overlap function of the $\mathrm{Cu}_{2} \mathrm{O}$ band gap and SPR used in fitting the relative number of carriers. 
Transient-Absorption Spectroscopy. Photocatalysis spectra are a result of carriers that have reached the surface of the $\mathrm{Cu}_{2} \mathrm{O}$ nanoparticle and contribute to the photo-degradation of the reactant. The photocatalysis spectra do not directly show how the carriers are created. Both RET and DET can create carriers in the trap and defect states in $\mathrm{Cu}_{2} \mathrm{O}$, which may be inefficient in photocatalysis, such as the states present at $550 \mathrm{~nm}$, distorting the spectral efficiency seen in the action spectrum measurements. To further investigate the plasmon-mediated energy-transfer mechanism, transient-absorption spectroscopy was performed on the $\mathrm{Au} @ \mathrm{Cu}_{2} \mathrm{O}$ core-shell and the $\mathrm{Au} @ \mathrm{SiO}_{2} @ \mathrm{Cu}_{2} \mathrm{O}$ nanoparticles to monitor the carrier dynamics and relaxation times (Figure 4.5). In these pump-probe measurements, the samples were dried films and 100-fs pulses excite the plasmon and probe the carriers created in the conduction band of the $\mathrm{Cu}_{2} \mathrm{O}$ respectively. Pump pulses were varied in wavelength from $620 \mathrm{~nm}$ to $750 \mathrm{~nm}$ across the plasmon resonances and in fluence from $7 \mathrm{~mJ} / \mathrm{cm}^{2}$ to $15 \mathrm{~mJ} / \mathrm{cm}^{2}$. Carriers created in the $\mathrm{Cu}_{2} \mathrm{O}$ by plasmonic energy transfer were probed by an 800-nm pulse that transmits through the sample. Because the probe wavelength is longer than the wavelengths required for interband absorption or for plasmon excitation, the signal is due to free-carrier absorption in the conduction band of the $\mathrm{Cu}_{2} \mathrm{O}$ only. Since $\mathrm{Cu}_{2} \mathrm{O}$ is usually a p-type semiconductor, the transient absorption at $800 \mathrm{~nm}$ may include a contribution due to excited electrons or holes. However, to determine the energy transfer mechanism, only the relative number of electron hole pairs verse the wavelength is necessary, not the identity of the carriers probed. Hence, the relative change in transmission $|\Delta T / T|$ was directly proportional to the relative number of carriers created in the $\mathrm{Cu}_{2} \mathrm{O}$ by plasmonic energy transfer. Contributions to $|\Delta T / T|$ from the Au were excluded, based on short time-scale and low-amplitude response in transient absorption measurements performed on the uncoated cores. Typical transients for the core-shell and sandwich nanostructures are shown in Figure 4.5a, excited at $650 \mathrm{~nm}$ and low fluence. This wavelength was not at the peak of either plasmon resonance, but was further off resonance for the $\mathrm{Au} @ \mathrm{SiO}_{2} @ \mathrm{Cu}_{2} \mathrm{O}$, resulting in the lower signal amplitude.
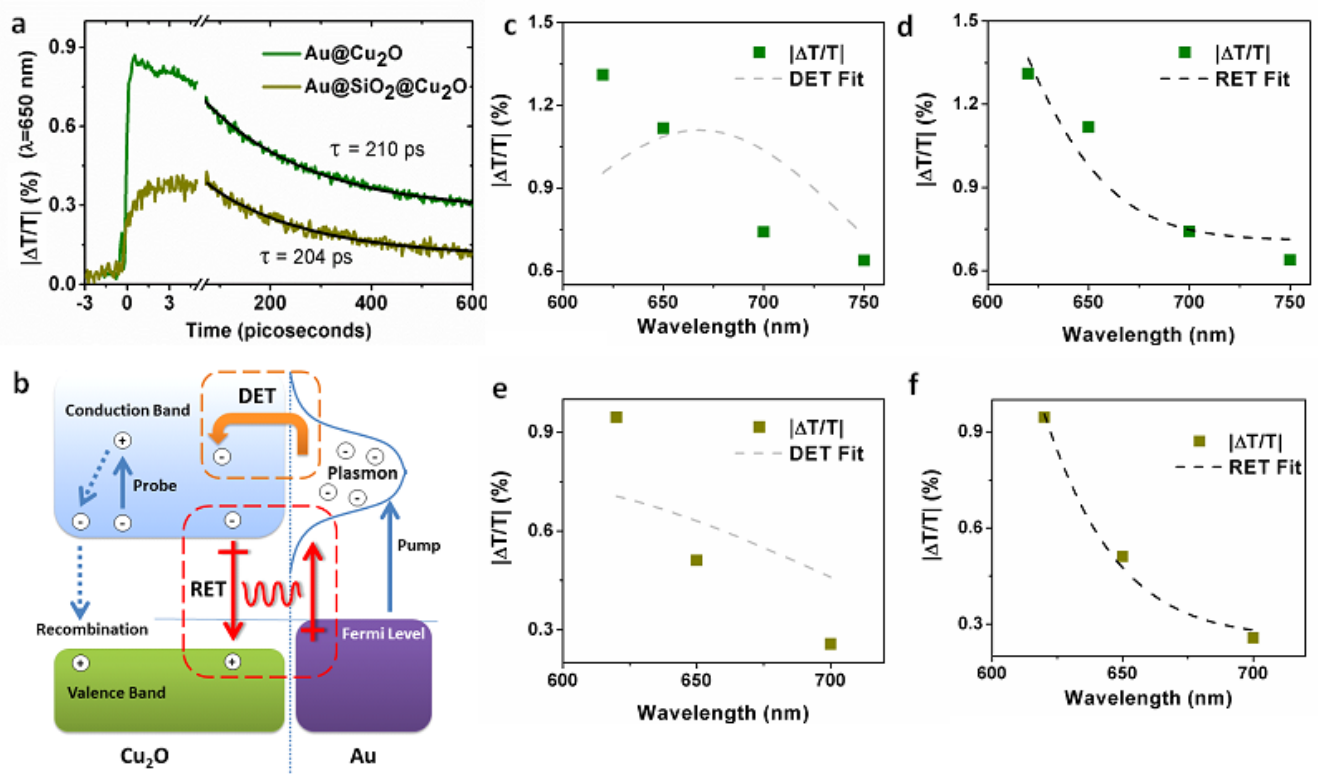

Figure 4.5. Ultrafast pump-probe measurements for core-shell and sandwich structures. a, Transient absorption for $\mathrm{Au} @ \mathrm{Cu}_{2} \mathrm{O}, \mathrm{Au} @ \mathrm{SiO}_{2} @ \mathrm{Cu}_{2} \mathrm{O}$ nanostructures acquired with a wavelength of $650 \mathrm{~nm}$ and a laser fluence of $7 \mathrm{~mJ} / \mathrm{cm}^{2}$. Decays are fit showing nearly identical recombination rates. b, Schematic representation of the various transfer mechanisms that can occur in the $\mathrm{Au} @ \mathrm{Cu}_{2} \mathrm{O}$ structure. Also shown in the diagram are the pump, probe (free-carrier absorption) and recombination paths. Wavelength -dependent signal amplitudes for $\mathrm{Au} @ \mathrm{Cu}_{2} \mathrm{O}$ nanoparticles are fit with c, DET and d, RET models. Similarly, e, DET and f, RET models are applied to experimental data from the Au@SiO $@ \mathrm{Cu}_{2} \mathrm{O}$ nanoparticle. 
The rise time of the signals from the $\mathrm{Au} @ \mathrm{Cu}_{2} \mathrm{O}$ has fast ( $\left.<100 \mathrm{fs}\right)$ and slow ( $\left.\sim 2 \mathrm{ps}\right)$ components, where the result of the fast excitation began to decay before the slower contribution has concluded. The fast component is most likely due to direct interactions in the metal. For $\mathrm{Au} @ \mathrm{SiO}_{2} @ \mathrm{Cu}_{2} \mathrm{O}$, only the slower rise component survives due to a simpler energy transfer scheme as might be expected by inserting the insulating layer into the structure. Carriers created in the $\mathrm{Cu}_{2} \mathrm{O}$ for both core-shell and sandwich structures were observed to have lifetimes on the order of a few hundred picoseconds, which was consistent with interband recombination. As the time delay is increased the population of free carriers that contribute to absorption decrease due to this recombination. The difference in the decay for the two samples was on the order of a few percent, which was only just outside the reproducibility of the experiment. These slight differences in the recombination time may arise from a non-radiative relaxation mechanism through the metal for the $\mathrm{Au} @ \mathrm{Cu}_{2} \mathrm{O}$ nanoparticles.

\subsubsection{Discussion}

Plasmon-induced charge separation mechanisms are illustrated in Figure 4.5b (DET and RET). RET describes the non-radiative transfer of energy by dipole-dipole interactions. The interaction strength depends on the separation distance between the dipoles and the overlap integral of the interacting dipoles' spectra (equation 4.3). RET and DET can be differentiated by the wavelength- and fluence-dependent carrier density, which is proportional to $|\Delta \mathrm{T} / \mathrm{T}|$. To ensure that $|\Delta \mathrm{T} / \mathrm{T}|$ only maps the transferred carrierdensity the signal was extracted at a time delay between the pump and probe of $\sim 10-15$ ps. In this time window all fast coherent dynamics, thermalization and Au signatures have occurred and recombination has not measurably decreased the contributing carrier density. Wavelength-dependent $|\Delta T / T|$ for $\mathrm{Au} @ \mathrm{Cu}_{2} \mathrm{O}$ (Figure 4.5(c,d)) and $\mathrm{Au@SiO}{ }_{2} @ \mathrm{Cu}_{2} \mathrm{O}$ (Figure 4.5(e,f)) nanoparticles were compared to DET and RET theory. Fluence dependence is shown in Figure 4.6.
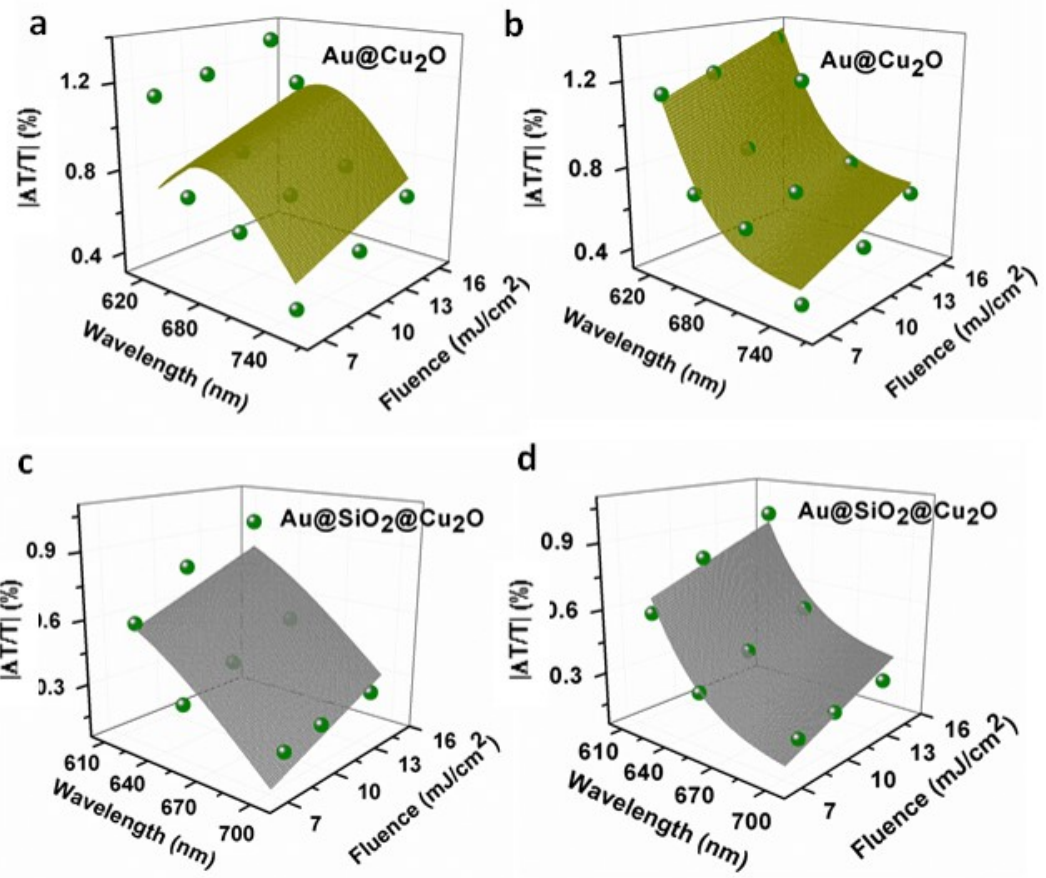

Figure 4.6. Fitting of Relative Number of Carriers for Several Powers. Fitting of plasmonic energy transfer mechanism

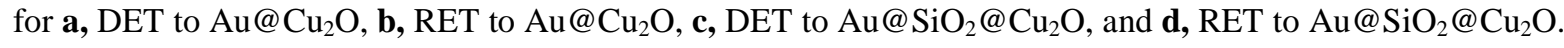


The excited plasmon produces a hot electron distribution which follows the plasmon's absorption for the small metal nanoparticles shown here (see Chapter 3). If these hot carriers transfer to the semiconductor, DET is the dominant plasmonic energy transfer mechanism, and the wavelength-dependent excited carrier density in the semiconductor will follow the lineshape of the plasmon absorption. To test DET theory, first the plasmon lineshape was extracted from the extinction spectrum (Figure 4.3b). A wavelength- and fluence-dependent surface was constructed from the Gaussian and assuming a linear dependence on fluence. The surface was used to fit the experimental data, leaving only the Gaussian amplitude used to fit the plasmon absorption and the slope of the fluence-dependence as free parameters. As can be seen from Figure 4.5c and 4.6a, the DET surface does not agree well with the experimental data, indicating that DET is not the correct transfer mechanism.

If RET is the dominant mechanism, the number of carriers created in the semiconductor conduction band obeys the dipole-dipole interactions for two distributions of dipoles (equation 4.1-4.3). ${ }^{10-14}$ Hence, the generated carriers will follow the overlap integral between the plasmon and the $\mathrm{Cu}_{2} \mathrm{O}$ density of states. $\mathrm{A}$ fitting procedure similar to that used for DET was applied to the RET mechanism, such that the Gaussian representing the plasmon is replaced by the overlap function (Figure 4.4), and then used to create a wavelength- and fluence-dependent surface. Only the slope of the fluence-dependence and amplitude of the overlap integral were free parameters in the fit. In contrast to the DET surface, the RET surface fit (Figure 4.5d and 5.6b) had excellent agreement to the experimental data, indicating that RET is the dominant mechanism for energy transfer from plasmonic $\mathrm{Au}$ to $\mathrm{Cu}_{2} \mathrm{O}$.

To verify the findings of RET as the mechanism for solar energy conversion from the plasmonic metal to the semiconductor conduction band, wavelength- and fluence-dependence tests were also performed on the $\mathrm{Au} @ \mathrm{SiO}_{2} @ \mathrm{Cu}_{2} \mathrm{O}$ sandwich nanostructure. In these structures the 5-nm thick $\mathrm{SiO}_{2}$ layer prevented DET. Note that if electron tunneling is present the signature would be clearly visible in the wavelength dependence. Hence, the only contribution should be RET, which is consistent with the simpler $\sim 2$ ps rise in $|\Delta \mathrm{T} / \mathrm{T}|$. As for the Au@ $\mathrm{SiO}_{2} @ \mathrm{Cu}_{2} \mathrm{O}$ sandwich samples, the wavelength- and fluence-dependent carrier density was extracted from the transient absorption and compared to surfaces for DET (Figure 4.5e and 4.6c) and RET (Figure 4.5f and 4.6d). The Gaussian distributions representing the plasmon resonance for DET and the overlap integral for RET were extracted from the extinction (Figure 4.4). In modeling, the fluence-dependent slope and the amplitudes of the various distributions remained the only free parameters. Once again, the RET model shows excellent agreement with the experimental results and the DET model does not, proving that RET is the dominant energy transfer mechanism for the $\mathrm{Au} @ \mathrm{SiO}_{2} @ \mathrm{Cu}_{2} \mathrm{O}$ nanostructures.

The transient-absorption and photocatalysis data show that the LSPR dipole creates electron-hole pairs in the semiconductor by RET. In particular, the overlap between the LSPR of the Au core and the interband absorption of $\mathrm{Cu}_{2} \mathrm{O}$ are well matched to demonstrate the RET process. To validate this conclusion and examine the exact form of the interaction term the transition rate was theoretically calculated using Fermi's golden rule. The final form of the calculation shows the dominant electromagnetic field mediated interaction between the semiconductor and LSPR is RET, similar in the short range asymptote to FRET.

The interaction of the semiconductor with the electromagnetic field near the valence band edge can be treated in the dipole approximation. It can be shown from the semiclassical light-matter interaction Hamiltonian that Bloch wave eigenstates are perturbed due to the electromagnetic field ${ }^{28}$

$$
H^{\prime}=\frac{e}{2 m c}(\vec{A} \bullet \vec{p}) \text {. }
$$


Through the commutator of position and momentum $H^{\prime}$ is equivalent to the dipole-field interaction Hamiltonian $^{29}$

$$
H^{\prime}=-\vec{\mu} \bullet \vec{E} .
$$

Hence, near the band edge, absorption in the semiconductor can be approximated by the interaction of a dipole-matrix element $\mu$ with the local electromagnetic field $E$. In the case of the sandwich nanoparticles, the semiconductor experiences an electromagnetic field composed of the incident radiation, $E_{0}$, and the dipole field of the LSPR, $E_{L S P R}$. The dipole moment of the LSPR can be calculated from Mie theory as ${ }^{30}$

$$
\mu_{L S P R}=\frac{\varepsilon_{\text {metal }}-\varepsilon_{\text {dielectric }}}{\varepsilon_{\text {metal }}+2 \varepsilon_{\text {dielectric }}} a^{3} E_{0},
$$

where $\varepsilon$ is the dielectric constant and $a$ is the metal nanoparticle radius. By inserting the electric dipole field of the LSPR into the light-matter interaction Hamiltonian for the semiconductor, the dipole-dipole interaction Hamiltonian is recovered, which leads to the familiar RET equation in agreement with the experimental data.

It has been proposed in previous reports that the EMF mechanism responsible for plasmon mediated charge separation could be a radiative or nonradiative interaction. The theoretical calculations and experimental data presented in this work indicate the EMF mechanism is solely RET, a nonradiative process. Quantum electrodynamics can be used to calculate the full interaction Hamiltonian between the dipole LSPR field and the dipole distribution in the semiconductor to determine if a radiative contribution is also present. A full quantum electrodynamics (QED) theory for the interaction of two dipoles by the electromagnetic field ${ }^{31}$ can be summarized in terms of the total transition rate, which is comprised of a RET transition rate, a far-field correction rate, and a radiative decay rate

$$
w_{\text {total }}=w_{R E T}+w_{\text {int }}+w_{\text {radiative }} \text {, }
$$

where

$$
\begin{aligned}
& w_{R E T}=\frac{9 \kappa^{2} c^{4}}{8 \pi \tau_{A^{*}} n^{4} r^{6}} \int F_{A}(\omega) \sigma_{B}(\omega) \frac{d \omega}{\omega^{4}}, \\
& w_{\text {int }}=\frac{9 c^{4}}{8 \pi \tau_{A^{*}} n^{2} r^{4}}\left(\kappa_{3}^{2}-\kappa_{1} \kappa_{3}\right) \int F_{A}(\omega) \sigma_{B}(\omega) \frac{d \omega}{\omega^{2}},
\end{aligned}
$$

and

$$
w_{\text {radiative }}=\frac{9 c^{4} \kappa_{1}^{2}}{8 \pi \tau_{A^{*}} r^{2}} \int F_{A}(\omega) \sigma_{B}(\omega) d \omega .
$$

$F_{A}(\omega)$ is the normalized fluorescence, $\sigma_{b}(\omega)$ is the absorption coefficient, $c$ is the speed of light, $n$ is the refractive index, $\kappa$ is an orientational factor as outlined in the supporting information, $r$ is the distance between the two dipoles, and $\tau_{A^{*}}$ is the radiative lifetime of $F_{A}$. In the near field, the full QED treatment is identical to the expression derived using Fermi's golden rule and the dipole-dipole interaction Hamiltonian for RET. ${ }^{31}$ No other near field terms arise from the calculation, so the plasmon mediated interaction mechanism must be the nonradiative RET and not a radiative term proportional to $\left|\mathrm{E}_{\mathrm{LSPR}} / \mathrm{E}_{0}\right|^{2}$. This is in agreement with the transient-absorption and photocatalysis measurements.

The enhancement of the transition probability due to the RET mechanism compared to the incident radiation $E_{0}$ can be estimated by calculating the value of $\sigma_{b}(\omega)$ and $F_{A}(\omega)$. The transition rate for the semiconductor, which is directly proportional to the absorption coefficient, is found using Fermi's golden rule and summing over all possible states assuming only direct transitions and a parabolic band gap ${ }^{28}$ 


$$
w_{\text {semi }}=\frac{e^{2}\left|E_{0}\right|^{2}}{4 \pi^{2} m^{2} \hbar \omega^{2}}\left|p_{0}\right|^{2}\left(\frac{2 m_{\text {reduced }}}{\hbar^{2}}\right)^{3 / 2}\left(\hbar \omega-E_{g}\right)^{1 / 2}
$$

where $m$ is the electron mass, $m_{\text {reduced }}$ is the reduced mass of the electron and hole, $p_{0}$ is the momentum matrix element in the semiconductor, and $\mathrm{Eg}$ is the band gap energy of the semiconductor in $\mathrm{eV}$. The function $F_{A}(\omega)$ can be estimated by using the dipole moment of the LSPR from Mie theory and an area normalized Lorentzian $f(\omega) .{ }^{30}$ The ratio between the probability of exciting an electron hole pair in the semiconductor by RET and by the incident field becomes

$$
\frac{w_{R E T}}{w_{\text {semi }}}=\frac{20 \pi \kappa^{2} \hbar \omega^{2}}{n^{5}} \frac{a^{6}}{r^{6}}\left|\frac{\varepsilon_{\text {metal }}-\varepsilon_{\text {die }}}{\varepsilon_{\text {metal }}+2 \varepsilon_{\text {die }}}\right| \frac{\int f(\omega)\left(\hbar \omega-E_{g}\right)^{1 / 2} \frac{d \omega}{\omega^{2}}}{\left(\hbar \omega-E_{g}\right)^{1 / 2}},
$$

where the frequency dependence of $\varepsilon$ and $p_{0}$ have been ignored as a first approximation. The above must be integrated over the distance of interaction $r$ and the range of frequencies $\omega$ to see the full enhancement, but can be approximated by taking $r=a$ and evaluating the overlap integral numerically. Assuming a band gap of $600 \mathrm{~nm}$ and a LSPR centered at $600 \mathrm{~nm}$ with a half width of $100 \mathrm{~nm}$ for a gold nanoparticle (with $\varepsilon_{\text {metal }}$ roughly negative $2 \varepsilon_{\text {dielectric }}$ as required for LSPR), the enhancement is approximately 10,000 . The value will obviously increase at values of $r<a$ due to the $1 / r^{6}$ term. The large increase in transition probability allows optically inefficient band edge, surface, and defect states to effectively support photocatalysis. It has also been reported that the near-field interaction can allow indirect transitions and coupling to optically forbidden states. ${ }^{32,33}$

Förster resonance energy transfer (FRET) has been well studied in various systems such as organic dyedye, quantum dot-quantum dot, dye-gold nanoparticle, and quantum dot-gold nanoparticle systems. Herein it is proven that the local electromagnetic field mediates energy transfer through RET from a plasmonic metal to a semiconductor, inducing charge separation in the semiconductor. Conventional FRET in a dyedye system is a downward energy transfer process. In contrast, the newly discovered RET from a plasmonic metal to a semiconductor can be thought of as an effectively upward energy transfer process, allowing for the conversion of below band edge light into charge carriers in the semiconductor, as demonstrated in the present work. Of course energy is conserved and it's the overlap between broad energy resonances that allow for the seemingly upward energy transition.

Hence, a plasmonic metal nanostructure can act as a photosensitizer via the RET process. A photosensitizer's ability to extend a semiconductor's effective optical band gap with conversion efficiency equal to that in wavelength below the band gap could transform design in photocatalysts, photovoltaic devices and other optoelectronic devices. In addition, the light absorption of the plasmonic photosensitizer can be tuned from the visible-light to near-infrared wavelength range by tailoring the LSPR. Furthermore, RET from a plasmon resonance to a semiconductor does not require direct contact between the energy donor and acceptor. Given that the local electromagnetic field surrounding a plasmonic nanostructure can reach tens of nanometers, because the resonance distance in RET from a plasmonic metal to a semiconductor could be longer than the typical Förster distance $\left(R_{0}=6 \mathrm{~nm}\right.$ in conventional FRET).

Semiconductors are often coupled with conventional photosensitizers such as organic dyes and inorganic quantum dots to enable light harvesting in the visible-light or near-infrared regime. Such coupling is based on the direct electron transfer from the conduction band of the photosensitizer to the conduction band of the semiconductor. In order for the electron transfer to be energetically favorable the photosensitizer's conduction band must be higher in energy than the semiconductors. As well, to maintain charge equilibrium the valence band of the photosensitizer must also be electronically aligned to allow hole transfer. These conditions on band alignment place strict limits on material selection for efficient 
photosensitizers. In contrast, plasmonic photosensitizers based on the RET from a plasmonic metal to a semiconductor do not require electronic band alignment, offering much more flexibility for designing solar energy materials and optoelectronic devices.

\subsection{Plasmon-Induced Resonant Energy Transfer}

Balancing the semiconductor band gap against heating $\operatorname{losses}^{34}$ in photovoltaics and photocorrosion ${ }^{35}$ in photoelectrochemical cells limits absorption of the solar spectrum. Semiconductor-semiconductor heterostructures, hot carriers, and photosensitizers extend the absorption range by transferring charge between two dissimilar band gap materials, but band alignment and interfacial charge transfer issues limit the realizable enhancement. ${ }^{36-38}$ Optically extracting energy by non-radiative dipole-dipole transfer could overcome these problems. However, dipole-dipole coupling in Förster resonance energy transfer (FRET) occurs in the incoherent and downward energy-transfer limit to a red-shifted acceptor ${ }^{11-14}$, preventing charge separation to a higher band gap material.

It is not that dipole-dipole coupling cannot extend light absorption. While the dipole is coherent before the Stoke's shift, symmetric energy transfer is observed on short time scales for strong coupling between excitons and plasmons ${ }^{39}$, atomic vapor ${ }^{40}$, photosynthesis ${ }^{41}$, and spin-triplet excitons ${ }^{42}$. Unfortunately, these conditions are difficult to meet in candidate solar materials such as quantum dots and fluorophores, because the radiative lifetime is longer than thermal relaxation. Plasmons, however, have a large dipole moment when the collective electron oscillations are coherent, creating the possibility of strong blue-shifted transfer.

Plasmonics modulating the energy flow direction in dipole-dipole coupling opens a third possible process for plasmon-enhanced solar-energy harvesting besides light trapping (Chapter 2) and hot electron injection (Chapter 3). In this case, the plasmonic metal absorbs sunlight, then transfers the absorbed energy from a metal to a semiconductor via dipole-dipole coupling, generating electron-hole pairs below and near the semiconductor band-edge..$^{21,43-45}$ We denote this process as plasmon-induced resonance energy transfer (PIRET) to distinguish the specific energy transfer direction made possible by the plasmon from the more generalized FRET.

The underlying mechanism of PIRET, the origin of its efficiency, and its difference from FRET are investigated experimentally and theoretically in this Section ${ }^{46}$ after its discovery in Section 4.2. A modified FRET theory and a density matrix model are first developed to account for the plasmon's inhomogenous broadening, small shift between absorption and emission, field decay length, and short lived coherent response. The model predicts the coherence time as well as spectral and spatial overlap with the acceptor govern the energy transfer direction, which is then experimentally confirmed in $\mathrm{Au} @ \mathrm{SiO}_{2} @ \mathrm{Cu}_{2} \mathrm{O}$ heterojunctions using transient absorption spectroscopy and photocatalysis. PIRET is shown to create electron-hole pairs in $\mathrm{Cu}_{2} \mathrm{O}$ using near- and below-band gap energy with efficiency comparable to $\mathrm{Cu}_{2} \mathrm{O}$ excited above the band-edge, demonstrating plasmonics can extend a semiconductor's absorption by optical extraction instead of interfacial charge transfer.

(Please Note: In order to keep the main discussion of this section separated from the many calculation and characterization methods needed, the following discussion is split into a main text with Figure 4.\# and Equation 4.\# and a Supporting Calculations section with Figure S4.\# and Equation S4.\#. The figures and equations are still labelled consecutively as they appear in the thesis to prevent confusion, but the supporting information is located after the main discussion.) 


\subsubsection{Methods}

Synthesis of Au nanoparticles: Au nanoparticles were synthesized according to the Frens' method using sodium citrate reducing $\mathrm{HAuCl}_{4}$ solution. ${ }^{23,24}$ Briefly, $100 \mathrm{ml}$ of $0.01 \mathrm{wt} \% \mathrm{HAuCl}_{4}$ aqueous solution was boiled and then $2 \mathrm{ml} 1 \mathrm{wt} \%$ sodium citrate was added. The solution changed to wine-red color in several minutes, indicating the formation of Au nanoparticles. The solution was kept boiling for an additional 30min to assure complete reduction of $\mathrm{HAuCl}_{4}$ in to Au NPs and then cooled down to room temperature.

Synthesis of Au@SiO ${ }_{2}$ with different shell thickness: The $\mathrm{SiO}_{2}$ coating on the Au NPs followed Ref. 47 using sodium silicate as the $\mathrm{SiO}_{2}$ precursor. Typically, $200 \mu \mathrm{l}$ of $2.0 \mathrm{Mm}$ APTS aqueous solution was added into $20 \mathrm{~mL}$ as-prepared Au NPs and stirred for 30min. Then $0.54 \mathrm{wt} \%$ sodium silicate aqueous solution was added and stirred for 5 minutes at $95^{\circ} \mathrm{C}$. The thickness was controlled by varying growth time and $\mathrm{pH}$ value of the reaction solution.

Synthesis of $\mathrm{Au} @ \mathrm{Cu}_{2} \mathrm{O}$ and $\mathrm{Au} @ \mathrm{SiO}_{2} @ \mathrm{Cu}_{2} \mathrm{O}: \mathrm{Cu}_{2} \mathrm{O}$ coating of Au nanoparticles followed Ref. 48. Briefly, Au nanoparticles were dispersed in the $\mathrm{Cu}^{2+}$-SDS stock solution, followed by addition of $\mathrm{NaOH}$ and $\mathrm{NH}_{4} \mathrm{OHCl}$ solution, then aged for 2 hours. The final samples were collected by centrifuge and washed with DI water and ethanol. The sandwich structure was prepared similar to the two-layer core-shell, but with surface modification. Au@SiO 2 was dispersed in ethanol with APTMS and refluxed for 6 hours. APTMS modified $\mathrm{Au@SiO}$ was re-dispersed in the $\mathrm{Cu}^{2+}$-SDS stock solution, and then followed the $\mathrm{Au} @ \mathrm{Cu}_{2} \mathrm{O}$ procedure.

Synthesis of $\mathrm{Ag} @ \mathrm{Cu}_{2} \mathrm{O}$ nanoparticles: The synthesis of Ag nanoparticles followed Ref. 43 with sodium citrate as the reducing agent. $\mathrm{AgNO}_{3}$ aqueous solution was heated to a boil, and then $1 \mathrm{wt} \%$ sodium citrate aqueous solution was added. After boiling for 30 minutes, the resulting solution became gray-yellow, indicating formation of Ag nanoparticles. The synthesis of $\mathrm{Ag} @ \mathrm{Cu}_{2} \mathrm{O}$ then followed $\mathrm{Au} @ \mathrm{Cu}_{2} \mathrm{O}$.

Photocatalysis: The photocatalytic activity was measured by degradation of methyl orange (MO) under fourteen $8 \mathrm{~W}$ visible light lamps or a $300 \mathrm{~W}$ Xe lamp coupled to a monochromator. Typically, four standard samples dispersed in $100 \mathrm{~mL}$ of $15 \mathrm{mg} / \mathrm{L} \mathrm{MO}$, and then ultrasonicated for $15 \mathrm{~min}$ and stirred 3 hours in dark to reach adsorption/desorption equilibrium. The degradation percentage was calculated by $\left(\mathrm{C}_{0}-\mathrm{C}\right) / \mathrm{C}_{0}$, where $\mathrm{C}$ is the $\mathrm{MO}$ solution absorption at each time interval and $\mathrm{C}_{0}$ is the initial absorption, determined using a Shimadzu spectrophotometer. All photocatalysis data is shown in detail in Reference.

Transient Absorption and Time Resolved Fluorescence: Transient absorption measurements were made with a 100 -fs pulse $1 \mathrm{KHz}$ Ti:sapphire laser amplifier split into an optical parametric amplifier (OPA) and an $800 \mathrm{~nm}$ probe, with the OPA frequency doubled in $\beta$-barium borate for the pump. Alternatively, the OPA provided the $1400 \mathrm{~nm}$ probe pulse and the $800 \mathrm{~nm}$ beam was frequency doubled for the pump. The pump and probe spot sizes were $500 \mu \mathrm{m}$ and $200 \mu \mathrm{m}$ respectively. The pump intensity was $5 \times 10^{10} \mathrm{~W} / \mathrm{cm}^{2}$. The broad-band white light probe was created by focusing $800 \mathrm{~nm}$ light into sapphire, using a circular polarization to increase stability, and the OPA for pump wavelengths. The chirp was corrected numerically relative to a reference sample with an instantaneous rise. The samples were dispersed in a $\mathrm{KBr}$ matrix and averaged over several spots to ensure uniformity. Time resolved fluorescence (TRF) was done using a double monochromated spectrofluorometer and a $375 \mathrm{~nm}$ laser diode pump, emission was collected at 500 $\mathrm{nm}$, and the samples were dispersed in ethanol.

Computational Simulations: Additional information regarding the various calculations and simulations, such as the coherent and inhomogenous broadening effects, is found at the end of the Discussion under the Supporting Calculations Section. 


\subsubsection{Results}

Distinguishing PIRET from FRET The energy transfer potential between donor and acceptor dipoles is given by their dipole moments $\left(\mu_{D}\right.$ and $\left.\mu_{A}\right)$ and their separation distance $(R)^{13}$

$$
\mathrm{V}_{D A}=\frac{\kappa}{4 \pi \varepsilon_{0}} \frac{\mu_{D} \mu_{A}}{R^{3}},
$$

where $\kappa$ is an orientation factor. Due to the line broadening associated with the dipole's finite lifetime ${ }^{49}$ (Fig. 4.7), two dipoles with partially overlapping spectra can transfer energy. In the case of fluorophores, thermal relaxation of excited carriers is quicker than spontaneous emission, creating a Stoke's shift. The Stoke's shift prevents re-absorption of an acceptor's fluorescence by the donor (Fig. 4.8), only allowing one transfer event to the red-shifted direction. These conditions make dipole-dipole coupling by FRET incoherent, with the energy transfer rate $\left(w_{D A}\right)$ given by

$$
w_{D A}=\frac{1}{\tau_{D}}\left(\frac{R_{0}}{R}\right)^{6} \text {, }
$$

where the distance at which $50 \%$ of the energy is transferred $\left(R_{0}\right)$ is defined through the spectral overlap integral as

$$
R_{0}=\frac{9 \kappa^{2} c^{4}}{8 \pi} \int F_{D}(\omega) \sigma_{A}(\omega) \frac{d \omega}{n(\omega)^{4} \omega^{4}},
$$

with $F_{D}$ the normalized donor emission area, $\sigma_{A}$ the acceptor absorption coefficient, $c$ the speed of light, $n$ the refractive index, $\kappa^{2}$ averaged to $2 / 3$, and $\tau_{D}$ the donor lifetime.
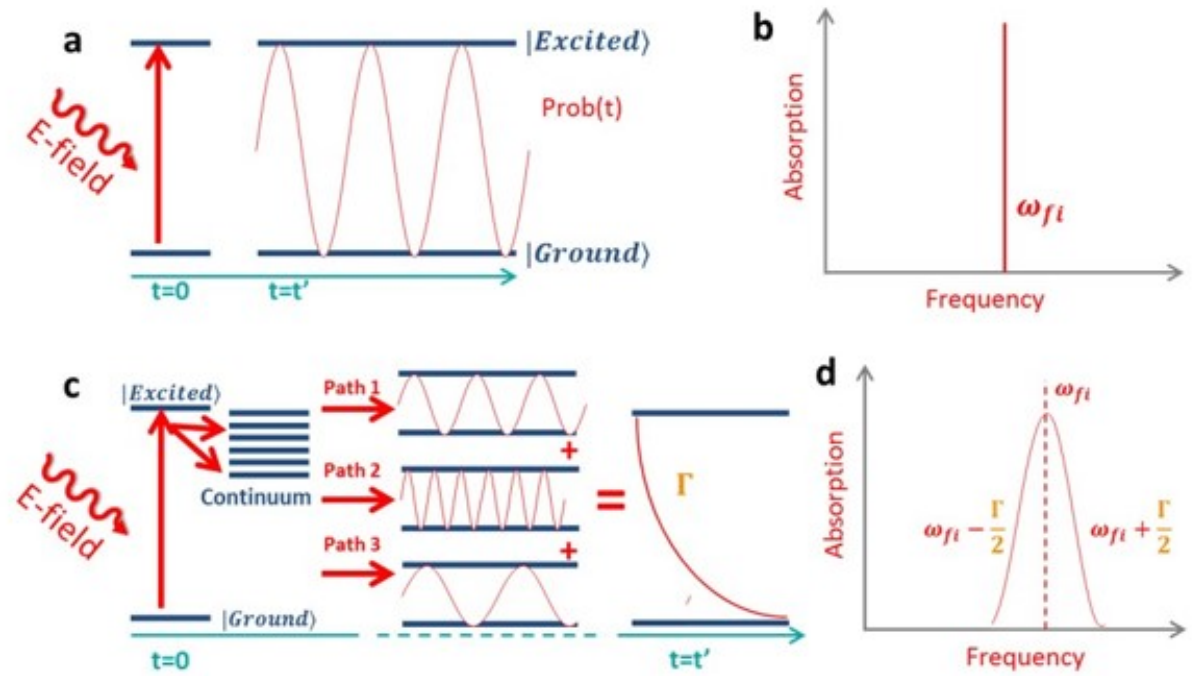

Figure 4.7. Response of a two state quantum system to an input field. a, When the two-state system can only couple to the incident field, a coherent superposition is excited between the ground and excited state which oscillates with a frequency equal to their difference in energy, $\omega_{f i}$. The state has an equal probability of being in the excited or ground energy level which oscillates with time. b, In the long time limit, the coherent oscillations lead to a discrete absorption frequency at $\omega_{f i}$. c, When the two-state system can couple to a continuum, such as by radiation or to a phonon bath, several quantum pathways are present with different phase and frequency after excitation. These pathways interfere, leading to a probability density that decays from the excited state to the ground state with time. $\mathbf{d}$, The finite lifetime of the system leads to a broadening of the discrete system's absorption. 
a Flourophore-Stoke's shift prevents PIRET

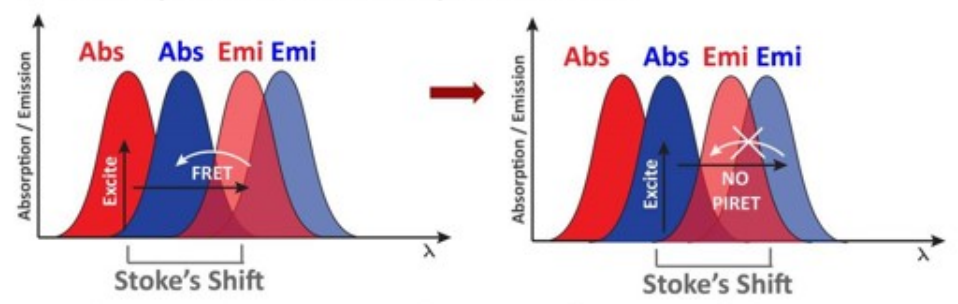

b Plasmon-absorption and emission (scattering) close

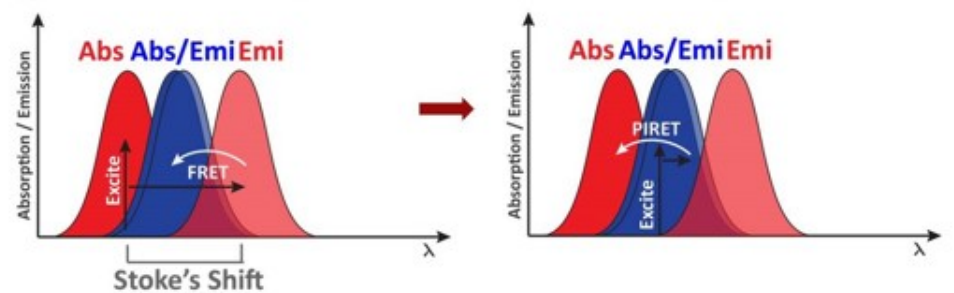

Figure 4.8. Effect of Stoke's shift in fluorophores on possible energy transfer. a, In a fluorophore, the large separation between the absorption dipole and emission dipole due to the Stoke's shift makes it difficult to simultaneously allow the overlap necessary for FRET from donor emissions to acceptor absorption and PIRET from acceptor emission to donor absorption. b, For plasmonic nanoparticles in the visible range the absorption and scattering are close in frequency, and therefore the necessary overlap can be maintained for FRET and PIRET.
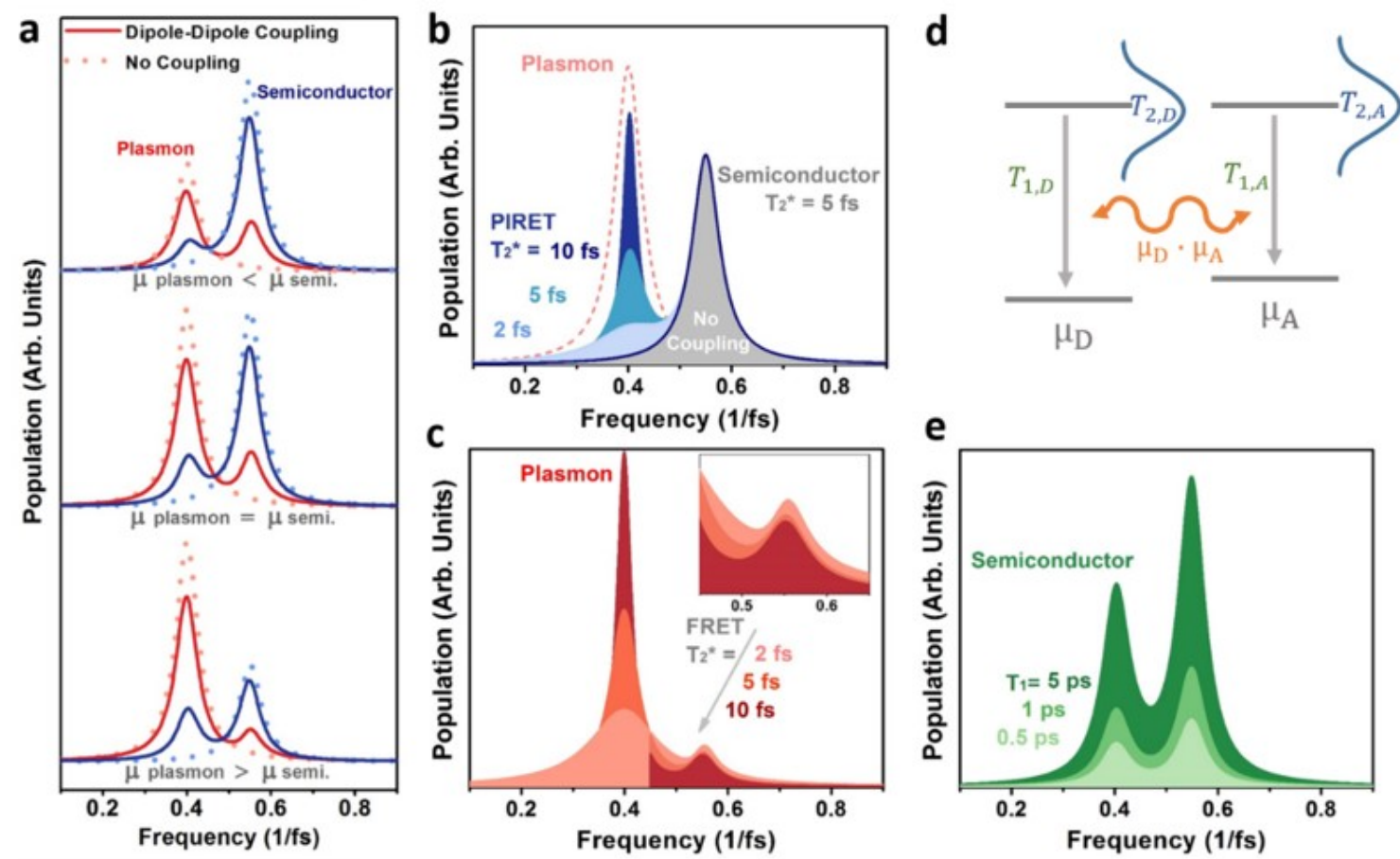

Figure 4.9. Coherent dipole-dipole coupling. a, Steady state population of the plasmon and semiconductor at long times depends on the relative dipole moments, which govern the balance between the semiconductor enhancing the plasmon's population (FRET in red) or vice versa (PIRET in blue). Populations without coupling are shown as dotted

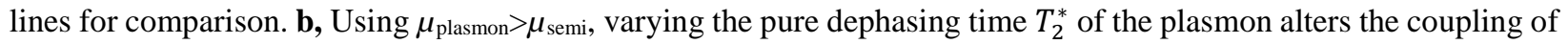
the plasmon to the semiconductor by PIRET, and $\mathbf{c}$ the coupling of the semiconductor to the plasmon by FRET. d, The density matrix includes population loss through recombination, $T_{1}$, and line broadening, $\frac{1}{T_{2}}=\frac{1}{2 T_{1}}+\frac{1}{T_{2}^{*}}$. e, Decreasing the recombination $T_{1}$ decreases the overall population in the semiconductor rather than the transfer direction or efficiency. 
The incoherent conditions of equation (4.14) do not hold for a plasmonic donor. The plasmon's large dipole moment originates in the collective motion of the many conduction electrons. When the individual electron's motions change phase relative to each other, for example by electron-electron scattering, the collective dipole moment decreases and the plasmon decays. Plasmonic dipole-dipole coupling must therefore occur before the Stoke's shift dephases the collective dipole moment, allowing blue or red-shifted energy transfer since absorption and emission can overlap. The energy transfer can also be coherent, with the plasmon continuing to give or accept energy from the other dipole through successive back and forward transfers until one of the dipoles dephases. Thermalization in the plasmon creates a non-equilibrium distribution of hot carriers which could also support a weaker, non-collective and incoherent dipole-dipole coupling.

To describe energy transfer with the plasmon as a donor, equation (4.14) and (4.15) were therefore replaced by a coherent formalism using the density matrix approach to solve the quantum master equation. ${ }^{50-56}$ The plasmon and semiconductor are represented by a four-level system (Fig. 4.9d) following Reference 50, comprised of a two-level donor and two-level acceptor. The plasmon is treated statistically in the density matrix, and the semiconductor represented by the sum of two-level interband transitions weighted by the joint density of states (JDOS). ${ }^{57}$ Diagonal elements are linear combinations of the two ground and excited states, while off-diagonal elements are coherences between these states developed during incident light and dipole-dipole interactions. The recombination time $\left(T_{1}\right)$ describes relaxation from the excited to ground state, and the dephasing time $\frac{1}{T_{2}}=\frac{1}{2 T_{1}}+\frac{1}{T_{2}^{*}}$ describes the decay of the coherences, where $T_{2}^{*}$ is pure dephasing. See Supplementary Calculations section for the input parameters used.

Figure 4.9a shows the population versus excitation frequency in the steady-state limit for the semiconductor and plasmon. The dashed curves show the excited state populations without dipole-dipole coupling. With coupling, exciting the plasmon (solid red) leads to population transfer to the semiconductor (solid blue) via PIRET, while exciting the semiconductor leads to population transfer to the plasmon via FRET. For two equal dipoles, center panel, energy transfer was mostly symmetric. When the red-shifted dipole's strength (plasmon) increased, it more strongly interacted with the incident light, breaking the symmetric interaction until population transfer to the blue-shifted dipole (semiconductor) approached the population from light absorption alone.

Next, the effect of dephasing time $\left(T_{2}\right)$ was determined for the example of $\mu_{\text {plasmon }}>\mu_{\text {semi, }}$, which showed a blue-shifted transfer. Unequal dephasing times lead to one dipole building up an incoherent population quicker during the coherent oscillations, trapping more energy on average. If the semiconductor's dephasing (5 fs) was faster than the plasmon's (10 fs), the largest PIRET population transfer was seen (Fig. 4.9b). Conversely, the largest FRET population transfer was seen (Fig. 4.9c) when the semiconductor's dephasing (5 fs) was slower than the plasmon's (2 fs), showing that when PIRET becomes less efficient, FRET becomes more efficient, conserving the overall population. Compared to the dephasing time $\left(T_{2}\right)$, the recombination time $\left(T_{1}\right)$ reduced the total population after transfer but did not affect transfer direction (Fig. 4.9e).

Dependence on Distance and Spectral Overlap For real materials, the semiconductor's interband transition dipole moment is an order of magnitude less than the plasmon's and the dephasing times are similar. ${ }^{57,58}$ Based on Fig. 4.9, PIRET population transfer below or near the semiconductor band-edge should be significant. The presence of FRET and PIRET were therefore experimentally confirmed in $\mathrm{Au} @ \mathrm{SiO}_{2} @ \mathrm{Cu}_{2} \mathrm{O}$ core-shell nanoparticles (Fig. 4.10). FRET and PIRET can be distinguished by separately 
exciting the coherent plasmon-to-semiconductor or incoherent semiconductor-to-plasmon energy transfer pathways (Fig. 4.11a-b). The dipole-dipole nature of the interaction was confirmed by measuring the population transfer to or from the semiconductor versus separation distance, controlled by varying the insulating $\mathrm{SiO}_{2}$ barrier thickness (Fig. 4.11c). The resulting distance-dependence was then predicted by the spectral overlap in equation (4.15). The core-shell geometry prevented other plasmonic enhancement pathways by blocking thermal and self-catalysis effects, hot electron transfer, and light scattering.
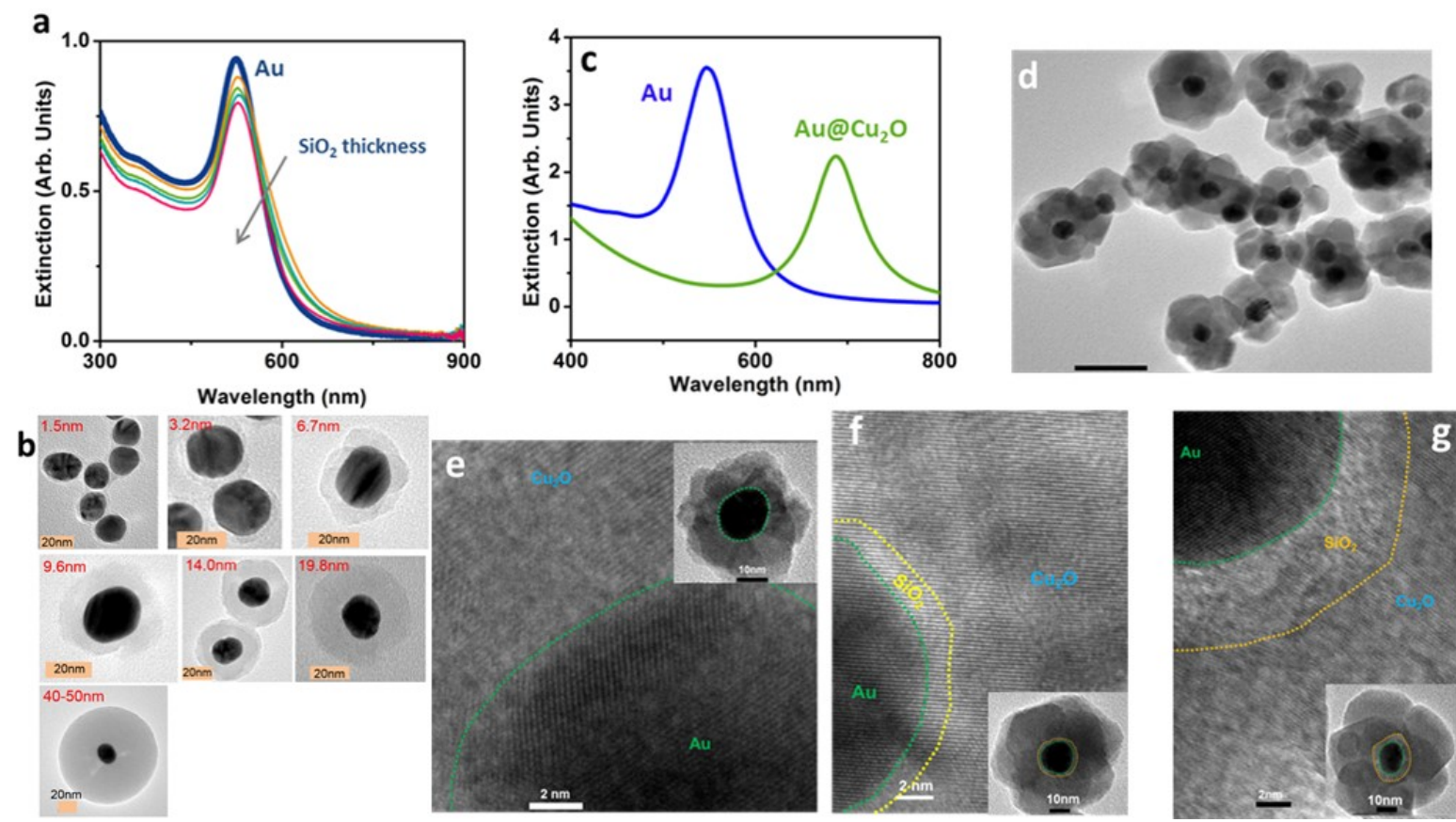

Figure 4.10. Synthesis and characterization of $\mathrm{Au} @ \mathrm{SiO}_{2} @ \mathrm{Cu}_{2} \mathrm{O}$ core shell nanoparticles. a, $\mathrm{The} \mathrm{SiO}_{2}$ shell results in negligible shifts of the gold nanoparticles LSPR peak from $530 \mathrm{~nm}$. b. TEM images showing the uniformity of the coated $\mathrm{SiO}_{2}$ spacer layer. c, After addition of the $\mathrm{Cu}_{2} \mathrm{O}$ shell Mie theory calculations predict a large shift but negligible broadening. The broadening in the experimental UV-Vis spectrum is therefore mainly a result of (d) the non-uniformity of the $\mathrm{Cu}_{2} \mathrm{O}$ shell. The shell is kept at $30 \mathrm{~nm}$ or larger to prevent incomplete coupling. TEM and HRTEM for (e) $\mathrm{Au} @ \mathrm{Cu}_{2} \mathrm{O},(\mathbf{f}) \mathrm{Au} @\left(1.5 \mathrm{~nm} \mathrm{SiO}_{2}\right) @ \mathrm{Cu}_{2} \mathrm{O}$, and $(\mathbf{g}) \mathrm{Au} @\left(7 \mathrm{~nm} \mathrm{SiO}_{2}\right) @ \mathrm{Cu}_{2} \mathrm{O}$. All scale bars in $(\mathbf{b})$ are $20 \mathrm{~nm}$, and the scale bar in (d) is $50 \mathrm{~nm}$.
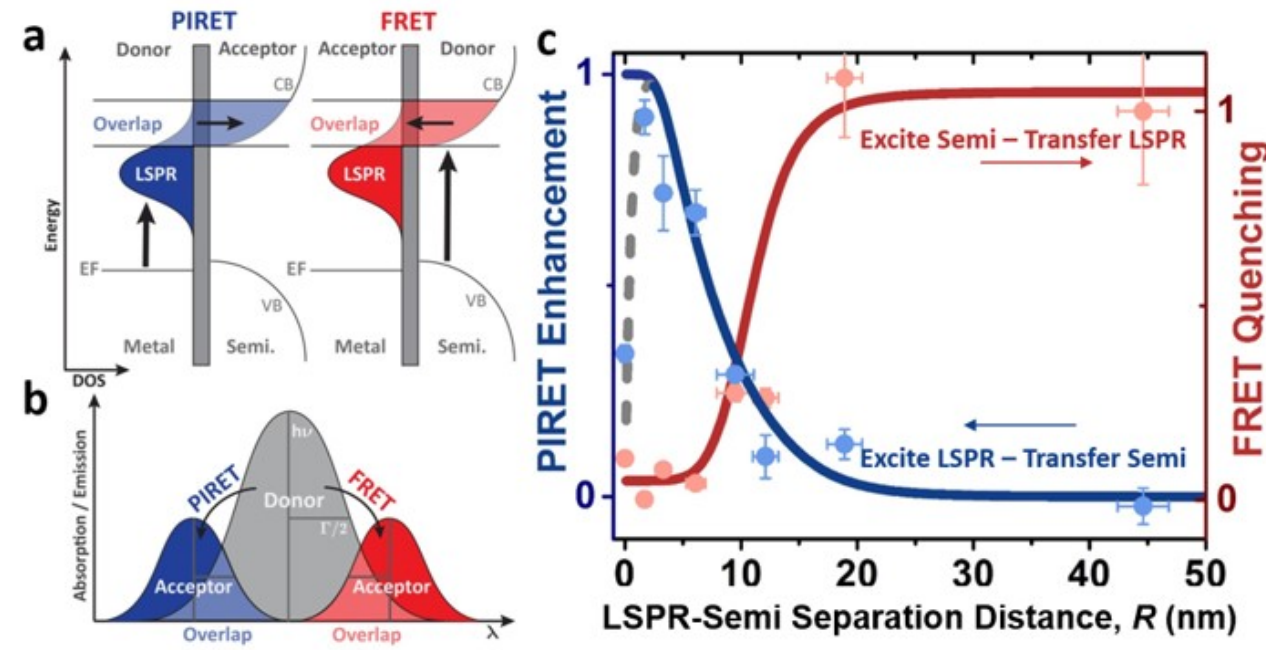

Figure 4.11. Complementary energy transfer with PIRET and FRET in $\mathrm{Au} @ \mathrm{SiO}_{2} @ \mathrm{Cu}_{2} \mathrm{O}$. a, For FRET, the $\mathrm{Cu}_{2} \mathrm{O}$ semiconductor is excited and its energy transfers to the plasmon. In PIRET, the plasmon is excited and its energy 
transfers to $\mathrm{Cu}_{2} \mathrm{O}$. b, The lifetime, $\Gamma$, of the system leads to a finite linewidth in the absorption and emission spectrum, allowing spectral overlap and dipole-dipole interactions for different central energies. c, The distance-dependence of the dipole-dipole interaction is similar whether the semiconductor is excited by PIRET or quenched by FRET. FRET quenching scale (defined in equation (S4.16)) is taken as one being no effect of the plasmon and zero being the semiconductor fully quenched, while the PIRET enhancement scale (defined in equation (S4.18)) goes from zero at no carriers added to the semiconductor to one at the peak plasmon-induced carrier creation.

The quenching of the semiconductor by the plasmon in FRET was determined by the change in fluorescence lifetime. The addition of carriers to the semiconductor by the plasmon in PIRET was determined by transient absorption. The experimental details and mathematical definition of FRET Quenching and PIRET Enhancement are further outlined in the Supplementary Calculations. Figure 4.11c shows that the FRET and PIRET distance-dependence were close to inversely symmetric because in FRET the plasmon took away excited carriers, but in PIRET the plasmon added excited carriers to the $\mathrm{Cu}_{2} \mathrm{O}$. Deviation occurred because of the different time scales involved for FRET and PIRET. FRET occurs incoherently from the thermalized population excited in $\mathrm{Cu}_{2} \mathrm{O}$ by ultraviolet (UV) light. Hence, the FRET data was fit excellently by equation (4.14), with the solid line showing the expected distance-dependent $\left(\frac{1}{R^{6}}\right)$ curve with $R_{0} \approx 11 \mathrm{~nm}$. PIRET occurs coherently, so equation (4.14) cannot be used directly. However, given coherent and incoherent theories converge in the steady-state and weak excitation limit satisfied here experimentally ${ }^{50}$, equation (4.14) can be modified for use with a plasmon.

First, the plasmon's absorption can be substituted into equation (4.15) since small metal nanoparticles with visible-light resonance have spectrally similar scattering, absorption, and transition dipole moments. ${ }^{59}$ The local field enhancement $\left(\left|E / E_{0}\right|\right)$ ratio from electromagnetic (EM) field simulations or the scattering could also be used, provided the pre-factors and $\omega^{-4}$ term are adjusted to relate the quantity back to the underlying transition dipole moment.

Second, the plasmon's field decay length relative to the conduction and valence band Bloch wavefunctions, which extend periodically over the semiconductor, invalidate the assumption of a spatially invariant EM field over the dipole in equation (4.13). ${ }^{60}$ Accordingly, too thin of $\mathrm{Cu}_{2} \mathrm{O}$ shells relative to the plasmon's field decay result in incomplete coupling, so a $>30 \mathrm{~nm} \mathrm{Cu}_{2} \mathrm{O}$ thickness was used for all samples.
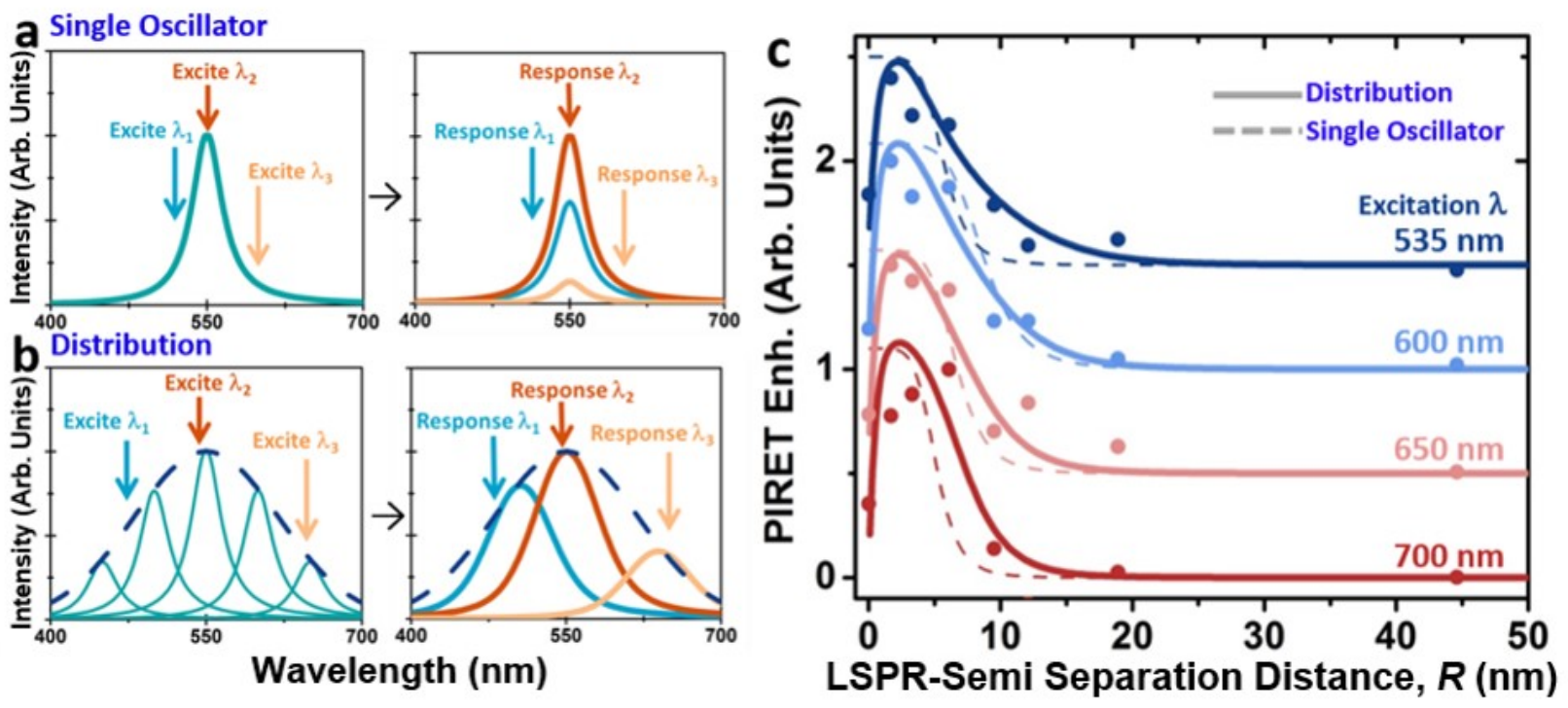

Figure 4.12. PIRET coupling with distance and wavelength for $\mathrm{Au} @ \mathrm{SiO}_{2} @ \mathrm{Cu}_{2} \mathrm{O}$. a, If a single oscillator is present, which could be a dipole representing a plasmon, off-resonance excitation by a narrowband source creates a population amplitude proportional to the absorption cross section. b, In a distribution, a given excitation only excites a subset of 
the oscillators, leading to a response that is distorted compared to the full ensemble absorption or emission. c, In the samples, the plasmon response is an ensemble, meaning that different excitation wavelengths lead to different overlaps between the plasmon and semiconductor, different values for $R_{0}$, and a modified distance-dependence. Symbols are experimental data and solid/dashed lines the distribution/single oscillator prediction. The PIRET enhancement is defined in equation (S4.18).

Third, the inherent size distribution in synthesized samples means the plasmon absorption in equation (4.15) must include inhomogenous broadening. The theoretical values for $F_{\text {plasmon }}$ and $\sigma_{\text {semiconductor }}$ were determined by deconvolving the $\mathrm{Cu}_{2} \mathrm{O}$ 's interband absorption, plasmon distribution, and particle Mie scattering contributions from the UV-Visible absorption spectra (Fig. S4.22). Inhomogenous broadening was then included by an ensemble average of individual linewidths estimated from Mie theory and discrete dipole approximation simulations (Figure S4.20), outlined in the Supplementary Calculations.
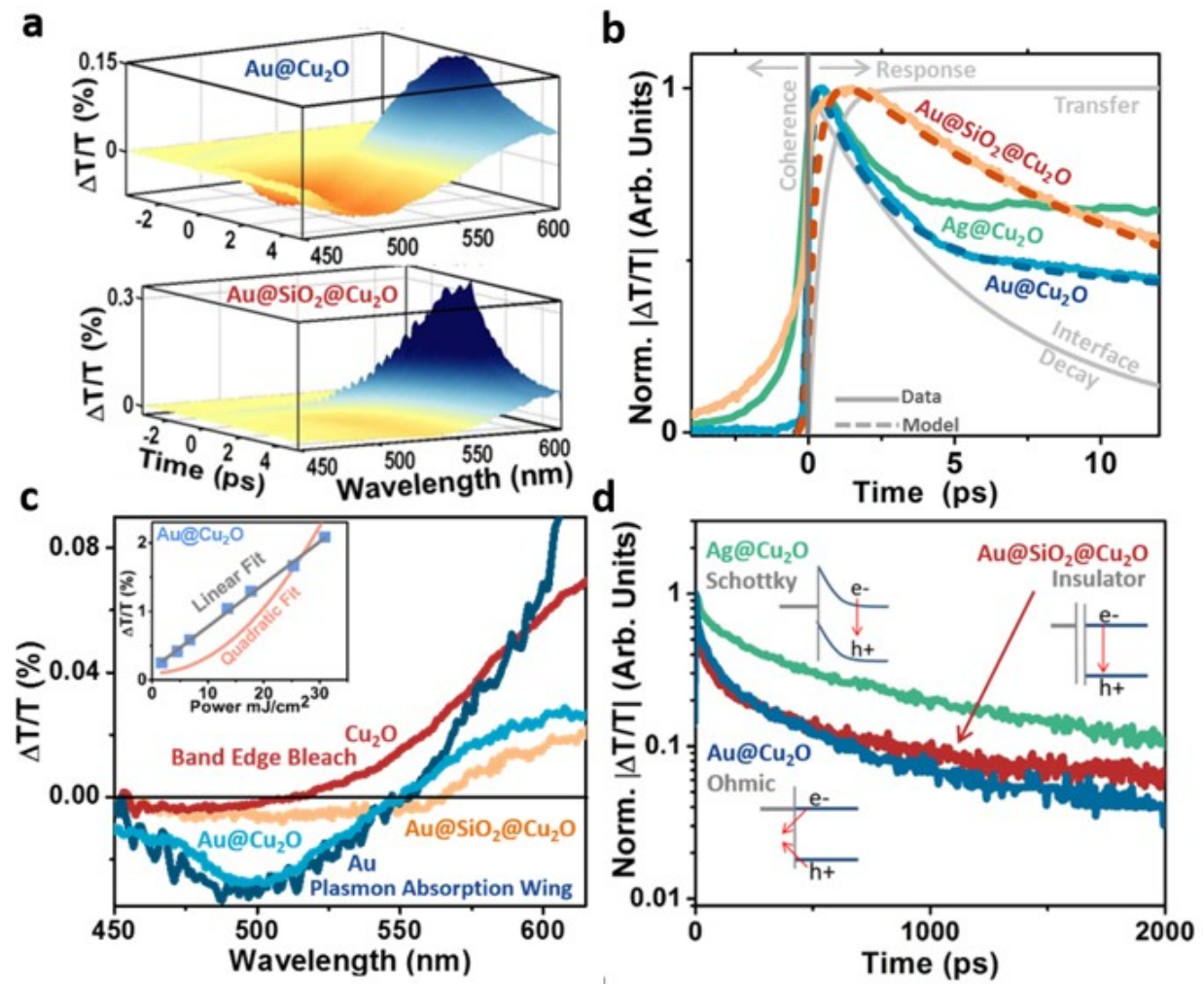

Figure 4.13. Transient dynamics of PIRET. a, Probe wavelengths below the band-edge reveal oscillations from the scattering of the pump off the coherence set up by the chirped probe in $\mathrm{Au} @ \mathrm{SiO}_{2} @ \mathrm{Cu}_{2} \mathrm{O}$, which disappear in $\mathrm{Au} @ \mathrm{Cu}_{2} \mathrm{O}$. b, The Au@SiO${ }_{2} @ \mathrm{Cu}_{2} \mathrm{O}$ transient absorption for exciting the plasmon and measuring the carriers transferred to $\mathrm{Cu}_{2} \mathrm{O}$ shows a long rise time from coherence which disappears when the spacer layer is removed. $\mathbf{c}, \mathrm{A}$ slice from the probe wavelengths at 10 ps after excitation reveals the plasmon in the $\mathrm{Au} @ \mathrm{SiO}_{2} @ \mathrm{Cu}_{2} \mathrm{O}$ is quenched, leaving only carriers in $\mathrm{Cu}_{2} \mathrm{O}$. The plasmon absorption wing returns in $\mathrm{Au} @ \mathrm{Cu}_{2} \mathrm{O}$ because increased dephasing results in less energy transfer. The inset shows linearity in excitation power. $\mathbf{d}$, The presence of the insulating barrier does not greatly affect long time scale dynamics, in contrast to the addition of a Schottky barrier in $\mathrm{Ag} @ \mathrm{Cu}_{2} \mathrm{O}$. The excitation wavelength in a-d is $650 \mathrm{~nm}$.

After these effects were taken into account, the distance-dependence in Fig. 4.11c for PIRET was fit excellently except for the direct metal-semiconductor contact (no $\mathrm{SiO}_{2}$ ). Prior to this discussion, which requires a full coherent treatment, the effect of inhomogenous broadening on the measured distancedependence is addressed. The spectral overlap integral (equation (4.15)) scales the distance-dependence (equation (4.14)), depending on the shape and strength of the plasmon's absorption. For a single metal 
nanoparticle, the plasmon's amplitude is weakened as the excitation wavelength is moved away from the homogenous linewidth's central wavelength (Fig. 4.12a). For an inhomogenous distribution, different homogenous lines are excited to different degrees depending on their detuning (Fig. 4.12b). This results in a shift of the spectral overlap when the distribution is summed over in a measurement, changing the apparent distance-dependence as the excitation wavelength is detuned from the distribution's center wavelength.

In the measured samples, the distribution was centered at 600-650 nm, depending on the $\mathrm{SiO}_{2}$ thickness (Fig. S4.15). The distance-dependence was therefore modified for $535 \mathrm{~nm}$ and $700 \mathrm{~nm}$ excitation (Fig. 4.12c), with the inhomogenous broadening model (solid line) allowing a smaller or larger subset of the plasmon oscillators to be excited relative to the semiconductor band-edge than the homogenous broadening model (dashed line), better describing the experimental trend. The measured ensemble distance-dependence is distorted closer to1/ $R^{4}$ in these cases (Fig. S4.23), however the underlying dipole-dipole coupling remains $1 / R^{6}$, similar to how geometrical effects change the apparent distance-dependence. ${ }^{61,62}$ For excitation near the plasmon's center, the homogenous and inhomogenous treatments agree, and the inhomogenous broadening allowed the shift in spectral overlap with the $\mathrm{SiO}_{2}$ thickness to be neglected.

Interfacial effects and coherence in PIRET When the plasmon and semiconductor are in contact, interfacial charge and back transfer should act to damp and dephase the plasmon quicker ${ }^{63}$, reducing PIRET as predicted in Fig. 4.9. On the other hand, the near Ohmic contact ${ }^{64}$ between $\mathrm{Au}$ and $\mathrm{Cu}_{2} \mathrm{O}$ should increase the interfacial recombination after transfer, also predicted to reduce the excited semiconductor population in Fig. 4.9e. Figure 4.13 shows the transient absorption spectroscopic data with and without metalsemiconductor contact, revealing that of these two effects, the dephasing is the critical parameter for determining below band-edge transfer efficiency and direction.

The coherence that developed during PIRET was evidenced by spectral fringing in the chirped broadband probe (Fig. 4.13a) and by perturbed free-induction ${ }^{65,66}$, which shows up as a long rise time before zero time delay (data in Fig. 4.13b, explained schematically in Fig. S4.24). The data is shown for Au@SiO $@ \mathrm{Cu}_{2} \mathrm{O}$ separated by 1.5 nm $\mathrm{SiO}_{2}$ where PIRET was strongest, but the trend is consistent for other $\mathrm{SiO}_{2}$ thicknesses (Fig. S4.25). The coherence delayed the creation of a population in the semiconductor after excitation of the plasmon, resulting in the broadened transfer function in Fig. 4.13b, and predicted by the density matrix model in Fig. S4.26.

When the insulating barrier was removed, forming $\mathrm{Au} @ \mathrm{Cu}_{2} \mathrm{O}$, the fringing and the long rise time vanished, consistent with a rapid reduction in the plasmon's coherence time $\left(T_{2}\right)$ due to interface damping (Fig. S4.26). Additionally, the transient's decay during the first few picoseconds after excitation was more pronounced, illustrating a new decay channel through the back transfer/interfacial recombination, modeled by the dashed lines as explained later in the Supplementary Calculations. Probing at energies near and above the $\mathrm{Cu}_{2} \mathrm{O}$ band-edge (Fig. 4.13c) revealed the competition of these two effects. Excitation of $\mathrm{Cu}_{2} \mathrm{O}$ alone (red) led to a bleach at $\sim 600 \mathrm{~nm}$, whereas excitation of the Au plasmon alone (dark blue) resulted in Fermi smearing ${ }^{67}$, and thus a bleach at the plasmon peak $(650 \mathrm{~nm})$ and absorption in its wings (500 nm). For $\mathrm{Au} @ \mathrm{SiO}_{2} @ \mathrm{Cu}_{2} \mathrm{O}$ (orange), PIRET is strong, and 10 ps after excitation, the plasmon had transferred most of its energy to $\mathrm{Cu}_{2} \mathrm{O}$, with only the $\mathrm{Cu}_{2} \mathrm{O}$ band-edge bleach and not the Au absorption being measured. For $\mathrm{Au} @ \mathrm{Cu}_{2} \mathrm{O}$ (blue), however, the increase in plasmon dephasing with metal-semiconductor contact led to reemergence of the plasmon absorption wings, showing more of the plasmon's energy was converted to hot electrons than transferring to $\mathrm{Cu}_{2} \mathrm{O}$.

Figure 4.13c therefore proves interface damping $\left(T_{2}\right)$ was dominant over recombination $\left(T_{1}\right)$ in reducing the PIRET efficiency. Recombination only reduced the semiconductor population after transfer and did not 


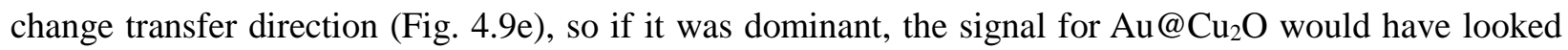
almost identical to $\mathrm{Au} @ \mathrm{SiO}_{2} @ \mathrm{Cu}_{2} \mathrm{O}$ but with reduced magnitude. Instead, the PIRET efficiency was seen to drop and the thermalized plasmon signal re-appear, consistent with interface damping changing the relative plasmon to semiconductor dephasing times (Fig. 4.9b). This conclusion was further confirmed by changing the Au core to Ag. Ag has a longer dephasing time than $\mathrm{Au}$, offsetting the decrease in dephasing from interface damping, and re-allowing PIRET in $\mathrm{Ag} @ \mathrm{Cu}_{2} \mathrm{O}$ as evidenced by return of the long transient rise time (Fig 4.13b). Further, the Schottky barrier in $\mathrm{Ag} @ \mathrm{Cu}_{2} \mathrm{O}$ was seen to reduce the interfacial recombination on short (Fig. 4.13b) and long (Fig. 4.13d) time scales.

Light absorption enhancement by energy transfer Fig 4.13c showed that the plasmon was quenched after energy transfer, indicating most energy absorbed by the plasmon had transferred to the semiconductor. Figure 4.14a shows that the high transfer efficiency persists near- and below-band-edge for photocatalysis as well as transient absorption, with PIRET extending the $\mathrm{Cu}_{2} \mathrm{O}$ photoconversion range without relying on charge transfer. For the smallest $\sim 1.5 \mathrm{~nm} \mathrm{SiO}$ barrier, PIRET excited at $650 \mathrm{~nm}$ created $\sim 1.4$ times the number of charge carriers in $\mathrm{Cu}_{2} \mathrm{O}$ as above-band gap excitation of the same incident flux, with the enhancement spanning the entire plasmon distribution. The enhancement decreased with increasing separation distance, in agreement with Fig. 4.12c. However, even for 7-nm separation, the near- and belowband-edge enhancement was still found significant for photocatalysis.

The transient signal or photocatalysis rate in Fig. 4.14a was normalized by carrier creation at $400 \mathrm{~nm}$ to determine the relative enhancement (equation (S4.19)). This normalization method was chosen over a ratio at the same excitation wavelength to highlight that PIRET can drive photoconversion near/below the bandedge at rates similar to the semiconductor above-band-edge. The experimental enhancement was consistent with the coherent density matrix model (Fig. 4.14b) as well as the simpler incoherent model (solid lines Fig. 4.14a), see Supplementary Calculations for parameters and implementation.
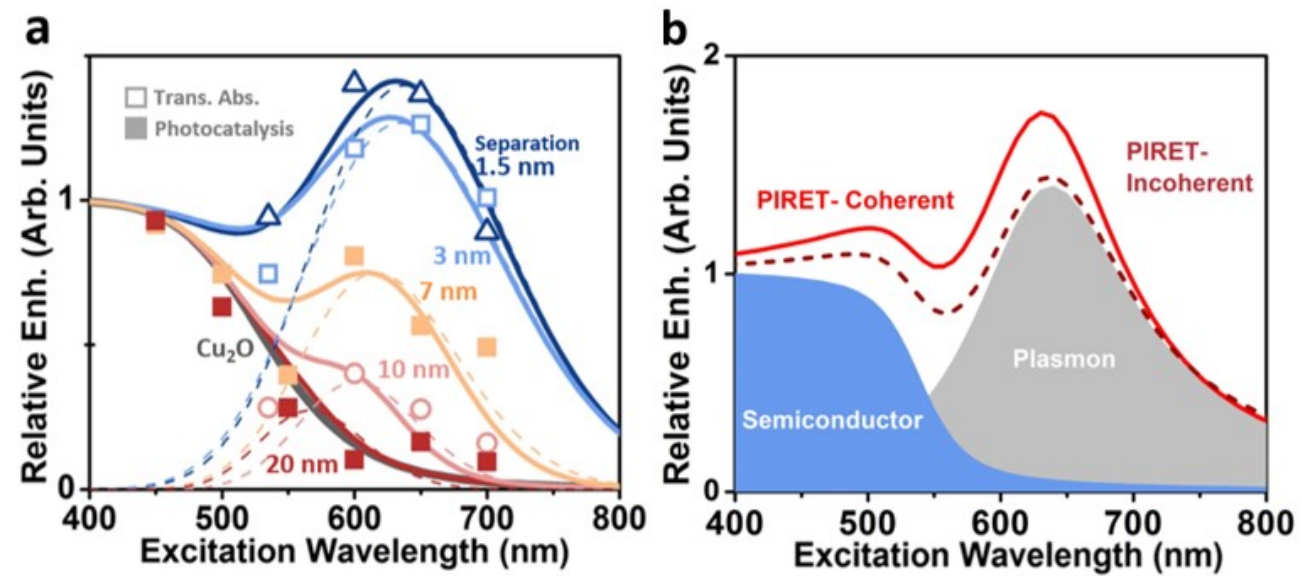

Figure 4.14. Enhancement of photoconversion by PIRET a, The relative PIRET enhancement (equation (S4.19)) follows the LSPR absorption, offset by the overlap with the semiconductor. Filled squares represent the enhancement measured by photocatalysis, empty shapes by transient absorption, and the solid lines are the predictions of the incoherent, inhomogenously broadened theory. $\mathbf{b}$, The theoretical magnitude of the relative enhancement, comparing the incoherent theory and the coherent density matrix model for the same semiconductor and plasmon parameters.

\subsubsection{Discussion}

Alternative explanations were investigated as follows, outlined in more detail in Supplementary Calculations. Two-photon absorption was ruled out since PIRET scaled linearly with increasing incident 


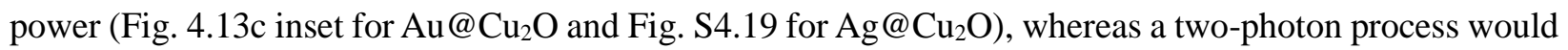
scale quadratically with incident power. Also, the predicted coupled two-photon absorption probability was $10^{-4}$ to $10^{-5}$ less than direct dipole-dipole coupling in the density matrix calculations used. The transient signal for PIRET followed that of above-band-edge excitation for $\mathrm{Cu}_{2} \mathrm{O}$ alone and not defect excitation below the band-edge (Fig. S4.17), ruling out defect state absorption. A two-temperature model (Fig. S4.18), the two dipole distance-dependence, and coherences ruled out phonon-assisted absorption. Second harmonic generation was also ruled out using the linearity of the signal with incident power.

Applying PIRET for sunlight harvesting requires several design considerations different from hot electron and light trapping enhancements. First, in Fig. 4.14, the relative efficiency will depend on the absorption of the $\mathrm{Cu}_{2} \mathrm{O}$ shell. A thick semiconductor shell would absorb more light, reducing the relative PIRET enhancement. PIRET is therefore best used in thin semiconductors, allowing reduced recombination losses and increased absorption within the plasmon's field decay length.

Second, the energy transfer is mentioned as blue or red-shifted in this paper relative to the central energy of the dipole, plasmon, or semiconductor band edge for clarity regarding the final effect on solar energy conversion. PIRET can only occur when spectral overlap is maintained, and is best used to increase population in a spectral region where the semiconductor absorbs weakly, such as near the band-edge.

Finally, while the plasmon-metal interface is traditionally only thought of as the Schottky barrier changing carrier lifetimes, the plasmon and semiconductor's relative dephasing times must be considered for efficient PIRET. As well, given the duality of PIRET and FRET, back-transfer of excited carriers by FRET on longer time scales must be removed. For example, the synthesized $\mathrm{Cu}_{2} \mathrm{O}$ had a fast non-radiative relaxation time and low radiative efficiency, trapping energy in the semiconductor after transfer. The largest enhancement can also occur when a thin insulating layer prevents the interfacial damping and recombination/back transfer.

\subsubsection{Supplementary Calculations}

PIRET/Relative Enhancement and FRET Quenching FRET quenching of the semiconductor was determined by extracting the fluorescence lifetime from the time-resolved fluorescence using the amplitude average of a three exponential fit (Table 4.1), including convolution with the instrument response. The carrier density by transient absorption spectroscopy (equation (S4.17)) was extracted 10 ps after excitation, after thermalization of transferred carriers but before significant recombination. FRET quenching is determined by taking the ratio of the amplitude averaged fluorescence lifetime with the plasmon versus that without dipole-dipole coupling at a $\mathrm{SiO}_{2}$ distance of $>50 \mathrm{~nm}$. PIRET Enhancement is normalized as one minus the ratio of the transient absorption signal in the $\mathrm{Cu}_{2} \mathrm{O}$ with the plasmon to the background signal of a $>50 \mathrm{~nm} \mathrm{SiO} 2$ barrier where dipole-dipole coupling is negligible. Relative Enhancement is defined similar to the PIRET Enhancement, but normalized by the photocatalysis or transient absorption signal for $400 \mathrm{~nm}$ excitation with the same flux. The methodology is further outlined in the Sub-Sections 2-3.

\section{Exclusion of Defects, Two-Photon Absorption, and Phonon-assisted Absorption}

The increase in absorption near/below the band-edge by PIRET could have a multitude of origins, each of which can be ruled out using the experimental and theoretical data presented.

i. Defects: The 800 -nm probe is sensitive to the defect states in $\mathrm{Cu}_{2} \mathrm{O}$ in both samples. To ensure the correct interpretation of where PIRET is depositing excited carriers in the $\mathrm{Cu}_{2} \mathrm{O}$, the wavelengthdependent response in the $\mathrm{Cu}_{2} \mathrm{O}$ is checked; see Fig. S4.17. For above-band gap excitation at 400 
$\mathrm{nm}$, the defect states are filled after fast relaxation resulting in a $\sim 2 \mathrm{~ns}$ lifetime. For near-band gap excitation at $550 \mathrm{~nm}$ the states partially bleach with a 10 ps lifetime followed by absorption with a significantly longer lifetime, similar to excitation at $400 \mathrm{~nm}$. For below-band gap excitation at $670 \mathrm{~nm}$, only bleaching is observed due to excitation from defects to the conduction band.

In the Au@SiO $2 @ \mathrm{Cu}_{2} \mathrm{O}$ samples, no bleach is observed when the plasmon is excited, Fig. S4.16, eliminating the possibility that PIRET is transferring into defect states or of a contribution from below-band-edge excitation in the $\mathrm{Cu}_{2} \mathrm{O}$ alone. A two-temperature model confirmed that the signal does not arise from the metal alone, see Fig. S4.18. Similar transient response of aboveband-edge excitation of the $\mathrm{Cu}_{2} \mathrm{O}$ and excitation of the plasmon confirms PIRET is transferring energy to the semiconductor to create carriers at the band-edge. The $\mathrm{Cu}_{2} \mathrm{O}$ alone in Fig. $4.13 \mathrm{c}$ was measured with four times the concentration of the other samples to create a detectable signal by below-band-edge excitation.

Two-photon absorption: The two-photon enhancement process could proceed through one of three ways:

First, the plasmonic field could enhance SHG in the $\mathrm{Cu}_{2} \mathrm{O}$, increasing absorption at belowband-edge energies. However, the $\mathrm{Cu}_{2} \mathrm{O}$ is centrosymmetric, and any SHG must therefore come from the interface or surface. Using Ref. 68 for an estimate from Si for the efficiency of surface SHG as $10^{-14} / \mathrm{W}$ with ultrafast pulses would require the plasmonic field to enhance by $\left|\mathrm{E} / \mathrm{E}_{0}\right| \sim 10^{7}$ (well above that of the nanospheres used, see Fig. S4.20) to allow equal carrier creation above and below the band-edge. Efficiency would be even lower for the CW photocatalysis, which was monochromated to $\sim 1 \mathrm{~mW}$. This mechanism can be further eliminated because plasmonic enhancement of two-photon absorption would have lead to a $1 / \mathrm{r}^{3}$ dependence on $\mathrm{SiO}_{2}$ thickness and extend to half the band gap energy. Moreover, two-photon absorption is a nearly simultaneous process that would not lead to a slow rise time.

Second, the SHG could be coming from the plasmon itself and then being absorbed by the $\mathrm{Cu}_{2} \mathrm{O}$. However, strong plasmon enhanced SHG can only occur for asymmetric structures (Ref. 69), or else the nonlinearity disappears except at the surface, limiting the SHG efficiency in the random distributions measured. A very strong local field enhancement would again be required, ruling out this effect. Further, the response would have no distance dependence or require an explanation including coherence.

Third, coherent two-photon absorption between the two dipoles could be suggested. However, using the density matrix calculations from Ref. 50, the simultaneous two photon absorption of both dipoles at $\omega_{1}-\omega_{2}-2 \omega=0$ is $10^{-4}$ to $10^{-5}$ less than the probability of exciting either dipole in the off-resonance and low field intensity case, safely excluding this effect as well.

The exclusion of all two-photon absorption effects is further supported by the linear excitation range measured in Fig. S4.19, which is consistent with past PIRET power dependencies in Section 4.2. The linear excitation range is further backed up by the equivalence of the measured relative enhancement at photocatalysis (milliwatts) and pulsed laser (megawatts) powers, as derived further in the section Possibility of Nonlinear Processes at the end of the Supplementary Calculations.

ii. $\quad$ Phonon-assisted Absorption: $\mathrm{Cu}_{2} \mathrm{O}$ is a direct band gap material, so any change in the absorption from phonons would have to be a phonon-assisted pathway, lowering the effective band gap by the energy of the phonon. This could occur by two ways: 
First, the heat of the metal or heat induced by the local field would increase the phonon bath in $\mathrm{Cu}_{2} \mathrm{O}$ incoherently, however, this would follow a $1 / \mathrm{r}^{3}$ dependence for the local field, and be maximum near the band-edge of the $\mathrm{Cu}_{2} \mathrm{O}$ and not at the peak of the plasmon. This explanation would also not account for the effects of coherence, the transients measured, and the phonon bath would be more efficiently excited when the plasmon had faster dephasing in the case of direct contact.

Second, phonon-assisted dipole-dipole coupling is commonly observed in molecular systems where coherent effects are important for energy transfer. Coherent phonons can increase the transfer rate if both dipoles share a common phonon bath or if the phonon energy matches the energy between the two dipoles. The temperature for crossover from the coherent to incoherent phonon-assisted process is estimated as $30 \mathrm{~K}$ for two molecular systems in the strong coupling region with a correlated bath, making these effects unlikely at room temperature. This is even less likely in the metal@insulator@semiconductor samples due to the separate phonon baths of the donor and acceptor. Further, transfer persists after the addition of the $\mathrm{SiO}_{2}$ spacer layers, the highest energy optical phonon in $\mathrm{Cu}_{2} \mathrm{O}$ would give a shift of $\sim 80 \mathrm{meV}(\sim 20 \mathrm{~nm})$ if populated requiring multi-phonon processes to account for the large shifts measured, and coherent phonon modes were not measured in the transients. All these results combine to make a higher-order coherent dipoledipole coupling mechanism hard to justify. Finally, no phonon affects are currently used in modeling the experimental data and good agreement is found.

\section{Measurement of Maximum and Relative Efficiency FRET and PIRET}

FRET quenching is experimentally determined as follows. Quenching of the $\mathrm{Cu}_{2} \mathrm{O}$ fluorescence lifetime by the plasmon was measured at $500 \mathrm{~nm}$ for a $375 \mathrm{~nm}$ excitation source and parameterized here by the amplitude averaged fluorescence lifetime for each sample, with the error bars relating to the error in the fit and shell thickness. The FRET quenching is taken as one minus equation (S4.20), which itself describes the change in the fluorescence lifetime from fully quenched to no quenching. This is recorded experimentally by

Quench $=1-E=1-\left[1-\frac{\tau_{\text {plasmon }+ \text { semiconductor }}}{\tau_{\text {semiconductor }}}\right]=\frac{\tau_{\text {plasmon }+ \text { semiconductor }}}{\tau_{\text {semiconductor }}}$

or one minus the standard equation used for FRET efficiency (Ref. 13). In equation (S4.16) $E$ is the efficiency given in equation (S4.20) and $\tau$ is the lifetime of semiconductor or plasmon/semiconductor system. The reference lifetime from the $50 \mathrm{~nm} \mathrm{SiO}{ }_{2}$ spacer was similar to that of the $\mathrm{Cu}_{2} \mathrm{O}$ alone, which was used as the value for $\tau_{\text {semiconductor }}$ to help remove background effects from the Au.

PIRET Enhancement is defined similarly to the FRET quenching, but instead of measuring the quenching of the fluorescent lifetime, the number of carriers excited in the semiconductor by the plasmon is recorded by transient absorption at $10 \mathrm{ps}$ after excitation. This approach is necessary as the donor (plasmon) lifetime cannot be directly measured from the $100 \mathrm{fs}$ pulses. The number of excited carriers is calculated from the transient transmission using

$\Delta N=-\frac{1}{\sigma d} \ln (1+\Delta T / T)$

where $\sigma$ is the absorption cross section for the probe, $d$ is the sample thickness, and $\Delta T / T$ is the transient signal. The PIRET enhancement can be normalized zero to one by 
$E=\frac{\Delta N(d)}{\Delta N_{\text {peak }}}-\Delta N_{o}$

where $\Delta N(d)$ is the number of carriers for a given $\mathrm{SiO}_{2}$ shell given by $E_{r e l}, \Delta N_{\text {peak }}$ is the peak number of carriers transferred for the excitation wavelength tested, and $\Delta N_{0}$ is the background at a large $\mathrm{SiO}_{2}$ spacer layer thickness. Error bars are determined from the average of three samples. 10 ps after excitation is chosen because at this point the excited carriers reach a long decay which was found representative of the photocatalysis performance, giving a good indicator as to the usable conversion efficiency.

Relative Enhancement is calculated as the number of charge carriers transferred into the $\mathrm{Cu}_{2} \mathrm{O}$ by PIRET at the excitation wavelength, divided by the number of carriers excited in $\mathrm{Cu}_{2} \mathrm{O}$ by a 400 -nm pump with the same incident flux. For a $650 \mathrm{~nm}$ pump, this gives the relative enhancement as follows

$$
E_{\text {rel }}=\frac{[\Delta N(10 p s, 650 \mathrm{~nm})]}{[\Delta N(10 p s, 400 \mathrm{~nm})]}=\frac{\left[\ln \left(1+\frac{\Delta T}{T}(10 p s, 650 \mathrm{~nm})\right)\right]}{\left[\ln \left(1+\frac{\Delta T}{T}(10 p s, 400 \mathrm{~nm})\right)\right]}
$$

The relative enhancement removes differences in particle concentration in the $\mathrm{KBr}$ matrix between samples, and the enhancements were scaled to account for pump power and spot size differences between excitation wavelengths. For photocatalysis, the same approach was taken, but instead normalizing by the photocatalytic rate at $400 \mathrm{~nm}$ compared to excitation of the plasmon. This is achieved by changing $\Delta N$ in equation (S4.19) to the photocatalysis rate for the appropriate excitation and normalization wavelength.

\section{Theoretical Definition of Maximum Efficiency FRET and PIRET}

From Ref. 13, the efficiency of dipole-dipole energy transfer can be written as

$$
E=\frac{w_{\text {dipole-dipole }}}{w_{\text {dipole-dipole }}+\sum w_{\text {other }}}=\frac{1}{1+\left(\frac{R}{R_{0}}\right)^{6}}
$$

where $w_{\text {dipole-dipole }}$ is the energy transfer rate, $w_{\text {other }}$ is the sum of competing transfer processes, $R$ is the dipoledipole separation distance, and $R_{0}$ is the distance at which $50 \%$ of the energy is transferred, defined by equation (S4.18). In the main paper, the FRET quenching efficiency was defined as

$$
E_{\text {quench }}=1-E
$$

such that a fully quenched emission has an efficiency of zero and the efficiency for the $50 \mathrm{~nm} \mathrm{SiO}_{2}$ thickness spacer is one. The $50 \mathrm{~nm}$ spacer is chosen as the references instead of $\mathrm{Cu}_{2} \mathrm{O}$ alone to help avoid the possibility of different non-radiative quenching rates due to the different interface. For PIRET, equation (S4.20) can be rewritten into a form that predicts the relative enhancement of carrier creation in the semiconductor with and without PIRET

$$
E_{\text {PIRET }}=\frac{\alpha_{\text {semi }}(\omega)+\alpha_{\text {LPSR }}(\omega) \cdot\left[\frac{1}{1+\left(\frac{R}{R_{0}}\right)^{6}}\right]}{\alpha_{\text {semi }}(\omega)}
$$

where $\alpha$ is the corresponding absorption coefficient. The change between equation (S4.20) and (S4.22) accounts for enhancement in carrier density in the $\mathrm{Cu}_{2} \mathrm{O}$ acceptor due to transfer from PIRET is being measured and not the quenching of the plasmon donor directly. Throughout the manuscript the pre-factor 
in equation (S4.22) is modified to give the PIRET enhancement relative to the peak conversion efficiency of the semiconductor, $\alpha_{\text {semi }}\left(\omega_{\text {peak }}\right)$, allowing a more realistic estimate of the enhancement in solar utilization. The small semiconductor absorbance near the band-edge in the denominator of equation (S4.22) will otherwise make the LSPR enhancement seem large, even though the overall effect on solar conversion efficiency is actually small.

\section{Electromagnetic Simulations}

Mie theory calculations were performed using MieLab (Ref. 70) and discrete dipole approximation (DDA) calculations using the open source DDSCAT (Ref. 25). The geometric parameters for the core shell nanoparticles were copied from the TEM images. In the DDA calculations a grid size of $0.1 \mathrm{~nm}$ was used and convergence was checked. The refractive index of $\mathrm{SiO}_{2}, \mathrm{Cu}_{2} \mathrm{O}$, and $\mathrm{Au}$ was taken from Palik and the online Sopra N and K database (Ref. 26).

\section{Quantum Master Equation and Density Matrix Model}

Quantum master equation calculations using the density matrix were done following Ref. 50 for both steady state and time dependent quantities. In this manuscript, the spin operators were used to calculate the excited state population of the semiconductor or plasmon as $\left\langle\frac{1}{2}+S_{z}^{i}\right\rangle$, corresponding to the diagonal elements of the density matrix. The recombination time $T_{1}$ and dephasing time $\frac{1}{T_{2}}=\frac{1}{2 T_{1}}+\frac{1}{T_{2}^{*}}$ were both included. The time dependent quantities were solved by numeric integration and the steady state quantities by solving the set of linear equations as outlined in Ref. 50. The excited state population of plasmon and semiconductor with and without coupling were then calculated versus excitation wavelength to determine the transfer. Many-particle effects are neglected in this approximation, especially after the plasmon decoheres into a hot carrier distribution. The cooperative emission term was neglected due to the close distance of the plasmon and semiconductor as justified in Ref. 50.

The input parameters for the full semiconductor model in Figure 4.14 were $\mu_{\text {plasmon }}=1.5 e-$ $27 \mathrm{Cm}$ estimated from Ref. 71; $\mu_{\text {semiconductor }}=1 e-28 \mathrm{Cm}$ estimated from Ref. 58 and roughly as the

charge times the lattice constant; $\tau_{\text {dephasing }}=3 f$ s for the plasmon based on the range of Ref. 72 plus the experimental inhomogenous linewidth and $\tau_{\text {dephasing }}=10 \mathrm{fs}$ for the semiconductor estimated from Ref. 57; and $\tau_{\text {lifetime }}=1$ ps based on average electron-phonon relaxation times for the metal and semiconductor from Ref. 67 and Ref. 73. The inhomogeneously broadened linewidth was used because the effect of the distribution was found negligible in predicting the excitation wavelength enhancement (Fig. 4.14b). An input field strength of $1 \mathrm{~mW}$ was used to assure the coherent effects still existed in the monochromated photocatalysis measurements. The dipole-dipole coupling was taken as $V=0.001 \mathrm{fs}^{-1}$ which for the dipole moments used corresponds to a center to center separation distance of $\sim 15-20 \mathrm{~nm}$, or a metal nanoparticle surface to semiconductor distance of $\sim 5-10 \mathrm{~nm}$. The semiconductor was treated as a weighted sum of dipoles over the JDOS of the semiconductor, based on the method of Ref. 57, and using the JDOS extracted from the experimental absorption. The relative enhancement was then calculated by finding the excited state semiconductor population versus excitation wavelength with dipole-dipole coupling to the plasmon, then normalizing against the excited state population without plasmonic enhancement.

Further parameters for individual figures are as follows: In the single dipole model of Figure 4.9, $\mu_{\text {semiconductor }}=1 e-27 \mathrm{Cm}, 1.5 e-27 \mathrm{Cm}, 2 e-27 \mathrm{Cm}$ in Part (a) with $\tau_{\text {dephasing }}=5 \mathrm{fs}$ for both 
semiconductor and plasmon dipoles and a dipole-dipole coupling of $V=0.01 \mathrm{fs}^{-1}$. In Part (b)-(d), $\mu_{\text {semiconductor }}=1 e-27 \mathrm{Cm}$ with dephasing as given in the Figure.

\section{Distribution Averaged PIRET Calculations}

The distribution averaged quantities shown in the manuscript were calculated as

$$
f=\int D\left(\omega, \omega_{g 0}, \delta\right) f(\omega) d \omega,
$$

where $f(\omega)$ is the average quantity to be calculated and $D\left(\omega, \omega_{g 0}, \delta\right)$ is the area normalized absorption distribution extracted from the UV-Vis absorption spectrum with center frequency $\omega_{g 0}$ and width $\delta$. $D\left(\omega, \omega_{g 0}, \delta\right)$ was either a lognormal distribution extracted from the UV-Visible data in the case of the relative enhancement versus wavelength or an averaged Gaussian in the case of the relative enhancement versus distance coupling data. For the distributions used, the lognormal distribution approaches a Gaussian due to its width. $D\left(\omega, \omega_{g 0}, \delta\right)$ is either

$$
\begin{aligned}
& \operatorname{Lognormal}\left(\omega, \omega_{g 0}, \delta\right)=\frac{1}{\omega \delta \sqrt{ }(2 \pi)} \exp \left(-\frac{\left(\ln \omega-\omega_{g o}\right)^{2}}{2 \delta^{2}}\right), \\
& \operatorname{Gauss}\left(\omega, \omega_{g 0}, \delta\right)=\frac{1}{\delta \sqrt{ }(2 \pi)} \exp \left[-\frac{\left(\omega-\omega_{g 0}\right)^{2}}{2 \delta^{2}}\right]
\end{aligned}
$$

where $\omega$ is the angular frequency, $\omega_{g 0}$ the center frequency of the distribution, and $\delta$ its width. Throughout the derivation $v$ refers to frequency, $\omega=2 \pi v$ is the angular frequency, and $\hbar \omega$ is energy.

\section{Frequency Response}

The spectral lineshape of a single particle, as calculated by Mie theory, is a Lorentzian with a width $\gamma$ and center $\omega_{\text {LO}}$. The frequency response needed to calculate the spectral overlap integral of a single oscillator can be thought of as the strength of the absorption at that excitation wavelength times the spectral width of the oscillator

$$
\operatorname{Resp}\left(\omega, \omega_{\text {exc }}\right)=\operatorname{Amp} \cdot L\left(\omega_{\text {exc }}, \omega_{L 0}, \gamma\right) \cdot L\left(\omega, \omega_{L 0}, \gamma\right) \text {, }
$$

where Amp is the peak absorption strength and

$$
L\left(\omega, \omega_{L 0}, \gamma\right)=\frac{\gamma^{2} \omega_{L 0}^{2}}{\left(\omega^{2}-\omega_{L 0}^{2}\right)^{2}+(\gamma \omega)^{2}},
$$

is the normalized spectral lineshape with $\gamma$ being the damping. In a distribution, this approach accounts for the spectral overlap and strength of each individual oscillator for a given excitation wavelength. The frequency response of the distribution to a single excitation wavelength is therefore the average of the sum of single particle responses

$$
\left\langle\operatorname{Resp}\left(\omega, \omega_{\text {exc }}\right)\right\rangle=A m p \cdot \int D\left(\omega^{\prime}, \omega_{g 0}, \delta\right) \cdot L\left(\omega_{e x c}, \omega_{L O}, \gamma\right) \cdot L\left(\omega, \omega^{\prime}, \gamma\right) d \omega^{\prime} .
$$

\section{Average Enhancement.}


Since each oscillator has a different local environment, and only one nanoparticle contributes to PIRET per shell because of the $30 \mathrm{~nm}$ shell thickness, all enhancement quantities must be averaged over the single particle values. The average $R_{0}$ distance for a single excitation wavelength is then, using angular frequency, $R_{0}\left(\omega_{\text {exc }}\right)=\int D\left(\omega, \omega_{g 0}, \delta\right) R_{0}\left(\omega, \omega_{\text {exc }}\right) d \omega$,

where

$$
R_{0}\left(\omega, \omega_{e x c}\right)=L\left(\omega_{e x c}, \omega_{0}, \gamma\right) \frac{9 \kappa^{2} c^{4}}{8 \pi} \int L\left(\omega_{e x c}, \omega_{0}, \gamma\right) \sigma_{\text {semi }}(\omega) \frac{d \omega}{n(\omega)^{4} \omega^{4}} .
$$

In equation (S4.30), from Ref. $13, \kappa^{2}=2 / 3$ is the orientational average of the dipole coupling, $\mathrm{c}$ is the speed of light, $n(\omega)$ the refractive index, and $\sigma_{\text {semi }}(\omega)$ is the semiconductor absorption cross section. Similarly, the average efficiency at a single excitation wavelength and a single semiconductor-plasmon distance is

$$
E_{\text {PIRET }}\left(R, \omega_{\text {exc }}\right)=\int D\left(\omega, \omega_{g 0}, \delta\right) E_{\text {PIRET }}\left(R, \omega, \omega_{\text {exc }}\right) d \omega,
$$

where

$$
E_{\text {PIRET }}\left(R, \omega, \omega_{\text {exc }}\right)=\frac{\alpha_{\text {semi }}\left(\omega_{\text {exc }}\right)+\alpha_{L P S R}\left(\omega_{\text {exc }}\right) \cdot\left[\frac{1}{1+\left(\frac{R}{R_{0}\left(\omega, \omega_{\text {exc }}\right)}\right)^{6}}\right] .}{\alpha_{\text {semi }}\left(\omega_{\text {exc }}\right)} .
$$

By using equation (S4.32) to calculate the average enhancement at each $\omega_{\text {exc }}$ of the solar spectrum the overall enhancement is found as used in Figure 4.14. Alternatively the distance dependence can be calculated at a single excitation wavelength by varying $R$, the semiconductor-plasmon distance, as used in Figure 4.11 and 4.12. The inhomogenous broadening allows the shift in LSPR distribution with thickness to be neglected, simplifying calculating the distance dependence curve, as only the response around the excitation wavelength is needed to predict the overlap integral. As a check, using the individual LSPR distributions did not change $R_{0}$ outside the error bars of the experiment.

\section{Two-Temperature Model}

The two-temperature model calculations were carried out identical to Reference 74. The coupling of the electron, lattice, and shell temperatures are described by the set of differential equations

$$
\begin{aligned}
& C_{e} \frac{\partial T_{e}}{\partial t}=-G\left(T_{e}-T_{l}\right)+P_{v o l}(t), \\
& C_{l} \frac{\partial T_{l}}{\partial t}=G\left(T_{e}-T_{l}\right)+\left.\frac{S}{V} \kappa_{\text {mat }} \frac{\partial T_{m a t}}{\partial r}\right|_{r=R},
\end{aligned}
$$

and

$$
\frac{\partial T_{m a t}}{\partial t}=D_{m a t} \frac{1}{r^{2}} \frac{\partial}{\partial r}\left(r^{2} \frac{\partial T_{m a t}}{\partial r}\right)-\left.\frac{S}{V} \kappa_{m a t} \frac{\partial T_{m a t}}{\partial r}\right|_{r=R} .
$$

In the coupled differential equations $C_{e}$ and $C_{l}$ are the electron and lattice heat capacities, $G$ is the electronphonon coupling constant, $T_{e}$ and $T_{l}$ are the electron and lattice temperature, $S / V$ is the surface to volume ratio of the metal nanoparticle, $R$ is the radius of the metal nanoparticle, $\kappa_{\text {mat }}$ is the matrix thermal 
conductivity, $T_{\text {mat }}$ is the temperature of the shell, and $D_{\text {mat }}$ is the heat diffusion coefficient of the shell. $P_{\text {vol }}(t)$ is the incident power absorbed per unit volume from the laser pulse. The values of all constants were taken from Ref. 74

The temperature is taken to be uniform inside the metal nanoparticle, which is a realistic approximation when comparing the excitation beam size, $\sim 500 \mu \mathrm{m}$, to the metal nanoparticle radius; see Ref. 74 . All three materials were taken to be at $300 \mathrm{~K}$ before excitation. The equations are solved in radial coordinates, with the matrix/air interface always held at $300 \mathrm{k}$. The transient absorption signal is calculated as

$$
\left|\frac{\Delta T}{T}\right|=\left|\frac{T_{e}(t)-T_{0}}{T_{0}}\right|
$$

With the initial pulse being a sech ${ }^{2}$ shape with a width of $100 \mathrm{fs}$ and an intensity of $5^{*} 10^{10} \mathrm{~W} / \mathrm{cm}^{2}$ identical to the experimental conditions.

The two-temperature model shows the electron relaxation proceeds by the following pathway. The electron bath first absorbs the energy as shown by equation (S4.33), then transfers the energy to the phonon bath of the metal by equation (S4.34). The boundary condition at $r=R$ allows heat transfer between the matrix and metal. After heat has been transferred into the matrix if diffuses away spatially as given by equation (S4.35). The electron-phonon cooling happens on a much quicker time scale than the phononphonon cooling, or in other words, the transfer of energy from equation (S4.33) to equation (S4.34) through $G\left(T_{e}-T_{l}\right)$ is much quicker than the transfer of energy through the phonon-phonon coupling at the interface given by $\left.\frac{S}{V} \kappa_{\text {mat }} \frac{\partial T_{\text {mat }}}{\partial r}\right|_{r=R}$. This implies that the initial decay will be independent of the material present at the interface or the phonon-phonon coupling mechanism. Although more accurate treatments of the surface transfer rate are possible, they only affect the long time scale phonon-phonon coupling; see Ref. 75.The electron-phonon coupling is independent, ruling this out as a background contribution to the enhancement measured by PIRET versus $\mathrm{SiO}_{2}$ layer thickness.

\section{1D Diffusion/Recombination Modeling}

The diffusion/recombination model was set up identical to that outlined in Ref. 76. Briefly, the diffusion and recombination of carriers was described by

$$
\frac{\partial \Delta n}{\partial t}=D_{a} \frac{\partial^{2} \Delta n}{\partial x^{2}}-\frac{\Delta n}{\tau}+G(x, t),
$$

where $\Delta n$ is the excess carrier concentration, $D_{a}$ is the ambipolar diffusion coefficient for $\mathrm{Cu}_{2} \mathrm{O}, G$ is the generation term, and $\tau$ is the recombination lifetime. The generation term was taken to be a sech ${ }^{2}$ pulse with an intensity of $5^{*} 10^{10} \mathrm{~W} / \mathrm{cm}^{2}$ identical to experiment and a width of $100 \mathrm{fs}$. The generation term creates a spatial distribution of carriers as $\frac{1}{R^{6}}$ inside the semiconductor shell. The diffusion equation was solved in one dimension, with the boundary condition at the $\mathrm{Cu}_{2} \mathrm{O}$ /air interface being a surface recombination term fit to the $\mathrm{Cu}_{2} \mathrm{O}$ decay measured experimentally. The boundary condition at the $\mathrm{Cu}_{2} \mathrm{O} / \mathrm{Au}$ or $\mathrm{Cu}_{2} \mathrm{O} / \mathrm{SiO}_{2}$ interface had the form of

$$
S R V \cdot \exp \left(-\frac{d_{S_{O_{2}}}}{\delta_{L P S R}}\right),
$$


where $S R V$ is the surface recombination velocity, fit to the transient decay of the $\mathrm{Au}_{0} @ \mathrm{Cu}_{2} \mathrm{O}$ with $d_{S i O 2}$ set to $0 \mathrm{~nm}$. The decay factor $\delta_{L S P R}$ was found to be $0.5 \mathrm{~nm}$ by fitting to the change in the initial decay time with spacer thickness. To predict the decay curve for the $1.5 \mathrm{~nm} \mathrm{SiO}_{2}$ spacer layer, $d_{\text {SiO2 }}$ was set to $1.5 \mathrm{~nm}$ and the calculation was repeated.

\section{Possibility of Nonlinear Processes}

Two photon absorption (TPA) or second harmonic generation (SHG) can be further ruled out since the same relative enhancement by PIRET is seen at both laser power (megawatts) and with a constant wavelength solar simulator after a monochromator (milliwatts). If TPA was dominant, this would imply that in the polarization $\chi^{(\mathrm{n})}$ had different values in different experiments for the same samples; however, $\chi^{(\mathrm{n})}$ must be constant with respect to field intensity, and therefore this scenario cannot occur.

This is proven by further examining the polarization, $P$. If a second-order process occurs the relative enhancement, which is experimentally normalized by the photoconversion measured through linear absorption above the band gap of the $\mathrm{Cu}_{2} \mathrm{O}$, would go as

$$
\begin{gathered}
E_{\text {rel }}=\frac{P_{\text {plasmon }+ \text { semi }}}{P_{\text {semi }}}=\frac{\chi_{\text {plasmon }}^{(2)} E_{1} C W E_{2} C W}{\chi_{\text {semi }}^{(1)} E_{1 C W}}=\frac{\chi_{\text {plasmon }}^{(2)}}{\chi_{\text {semi }}^{(1)}} E_{2} C W \\
\quad \neq \quad \\
\frac{\chi_{\text {plasmon }}^{(2)} E_{1 \text { laser }} E_{2} \text { laser }}{\chi_{\text {semi }}^{(1)} E_{1 \text { laser }}}=\frac{\chi_{\text {plasmon }}^{(2)}}{\chi_{\text {semi }}^{(1)}} E_{2} \text { laser }
\end{gathered}
$$

where $E_{r e l}$ is the relative enhancement and $E_{1,2}$ is the electric field for the CW photocatalysis or pulsed laser transient absorption. The experimental enhancement at both powers could only be constant if $\chi^{(2)}$ was changing between measurements, or if the intensity of the femtosecond pulsed laser equaled that of the constant wavelength solar simulator. The same conclusion holds for $\chi^{(3)}$ or any $\chi^{(\mathrm{n}>1)}$ process.

$$
\begin{aligned}
& E_{\text {rel }}=\frac{P_{\text {plasmon }+ \text { semi }}}{P_{\text {semi }}}=\frac{\chi_{\text {plasmon }}^{(\mathrm{n})} E_{1}{ }_{C W} \ldots E_{n_{C W}}}{\chi_{\text {semi }}^{(1)} E_{1 C W}}=\frac{\chi_{\text {plasmon }}^{(\mathrm{n})}}{\chi_{\text {semi }}^{(1)}} E_{2}{ }_{C W} \ldots E_{n_{C W}} \\
& \neq \\
& \frac{\chi_{\text {plasmon }}^{(\mathrm{n})} E_{1_{\text {laser }} \ldots E_{n_{\text {laser }}}}}{\chi_{\text {semi }}^{(1)} E_{1_{\text {laser }}}}=\frac{\chi_{\text {plasmon }}^{(\mathrm{n})}}{\chi_{\text {semi }}^{(1)}} E_{2_{\text {laser }} \ldots E_{n_{\text {laser }}}}
\end{aligned}
$$

In comparison, if a first order $\chi^{(1)}$ process like resonant energy transfer is present, the enhancement goes as

$$
\begin{gathered}
E_{\text {rel }}=\frac{P_{\text {plasmon }+ \text { semi }}}{P_{\text {semi }}}=\frac{\chi_{\text {plasmon }}^{(1)} E_{1 C W}}{\chi_{\text {semi }}^{(1)} E_{1 C W}}=\frac{\chi_{\text {plasmon }}^{(1)}}{\chi_{\text {semi }}^{(1)}} \\
\equiv \\
\frac{\chi_{\text {plasmon }}^{(1)} E_{1 \text { laser }}}{\chi_{\text {semi }}^{(1)} E_{1 \text { laser }}}=\frac{\chi_{\text {plasmon }}^{(1)}}{\chi_{\text {semi }}^{(1)}}
\end{gathered}
$$

in which case the two experimental enhancements measured at different power can agree, as seen when comparing the photocatalysis and transient absorption data. 


\section{Supplementary Figures}
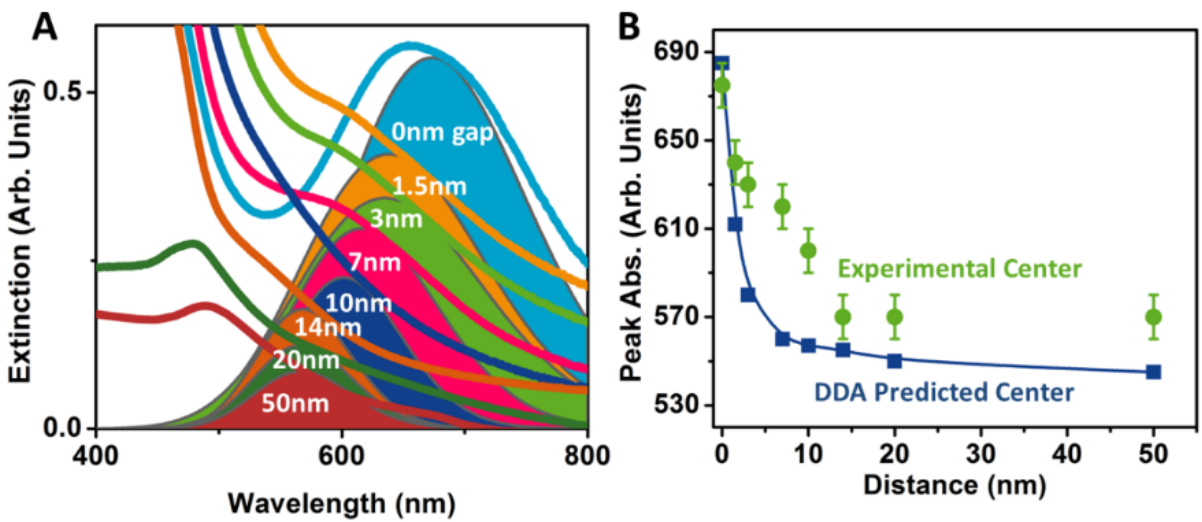

Figure S4.15. Extracted peak position for varying $\mathrm{SiO}_{2}$ layer thickness. a, A fit with the background components in Fig. S4.22 and the scattering predicted by Mie theory yields the peak position and band width of the LSPR distribution. The absorptions have been scaled and shifted for comparison of the peak position, however the plasmon is seen to always absorb less than the $\mathrm{Cu}_{2} \mathrm{O}$. b, The extracted peak position is seen to agree well with that predicted by single particle discrete dipole approximation (DDA) calculations, even for the large spacer thicknesses for which scattering dominates. The error bars are representative of the range over which the wide $\sim 150 \mathrm{~nm}$ distribution's peak position can vary while still maintaining a good fit.
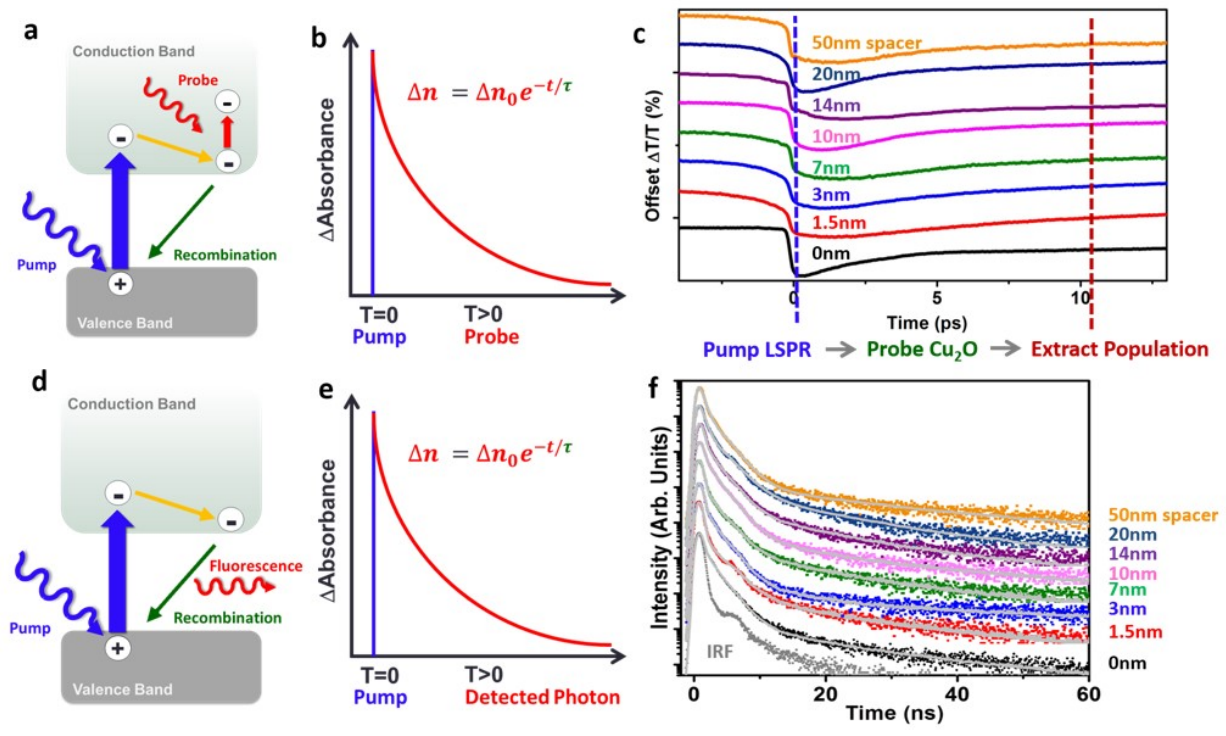

Figure S4.16. Transient absorption and time-resolved fluorescence in $\mathrm{Au} @ \mathrm{SiO}_{2} @ \mathrm{Cu}_{2} \mathrm{O}$. a, In a transient absorption measurement an intense pump pulse excites a carrier population. The excited carrier population absorbs the weaker probe pulse, with measurements (b) being taken at several time delays, mapping out the recombination lifetime of the excited carrier population. c, In the experiments shown for various $\mathrm{SiO}_{2}$ thicknesses, the LSPR is pumped at time $=0$, and then the carrier population in the $\mathrm{Cu}_{2} \mathrm{O}$ is probed at subsequent times. The carrier population excited by PIRET is then measured $\sim 10$ ps after time $=0$, after thermalization of carriers and interface recombination is complete but before significant long time scale recombination has set in. d, In time resolved fluorescence (TRF), the $\mathrm{Cu}_{2} \mathrm{O}$ is pumped, and then the lifetime is calculated by measuring the delay time between the pump and detected photons. e, By collecting the pump to detected photon time for a large number of photons, the lifetime of the spontaneous emission can be found. f, When the plasmon is present, dipole-dipole energy transfer occurs with increasing probability the closer the plasmon and semiconductor are, quenching the measured lifetime. The fit lines are shown in grey for each sample, the fit coefficients are given in Table 4.1. 

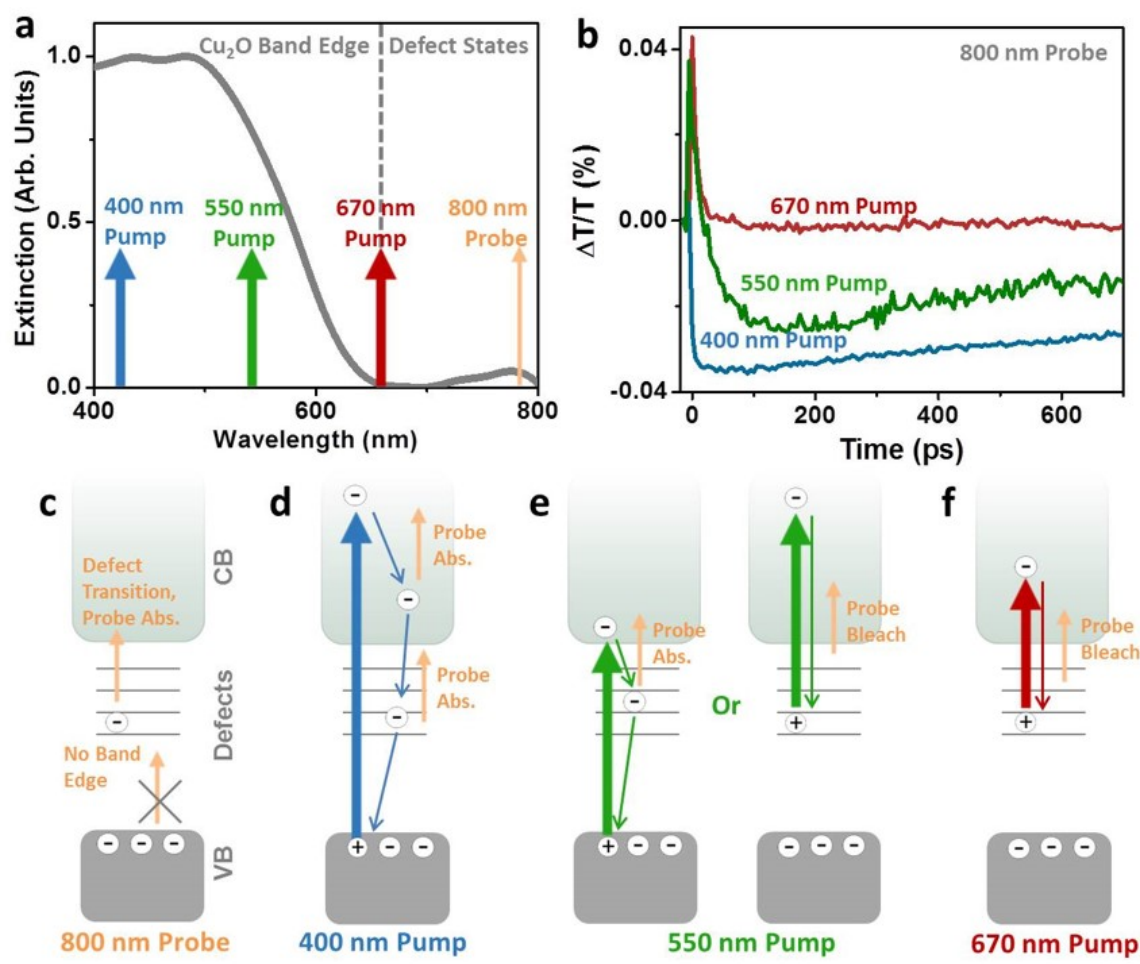

Figure S4.17. Decay processes in $\mathrm{Cu}_{2} \mathrm{O}$ for various pump wavelengths. a, Position of pump wavelengths relative to the $\mathrm{Cu}_{2} \mathrm{O}$ absorption. The dashed line shows the separation between band-edge states and defect state related absorption, also see (c). The probe is positioned at the defect state absorption at $800 \mathrm{~nm}$. b, For a $400 \mathrm{~nm}$ pump, an increase in absorption is found after excitation in the defect states, (d) indicating more carriers are present to absorb the $800 \mathrm{~nm}$ light. After a $550 \mathrm{~nm}$ pump, a quick bleach in the defect states is followed by an absorption, indicating (e) that the $550 \mathrm{~nm}$ pump can both excite band-edge transitions and intraband transitions, creating an absorption and bleach (less absorption), respectively. f, A $670 \mathrm{~nm}$ pump can only drive intraband transitions in the defect states, leading to (b) a short-lived bleach but no long lived carriers.
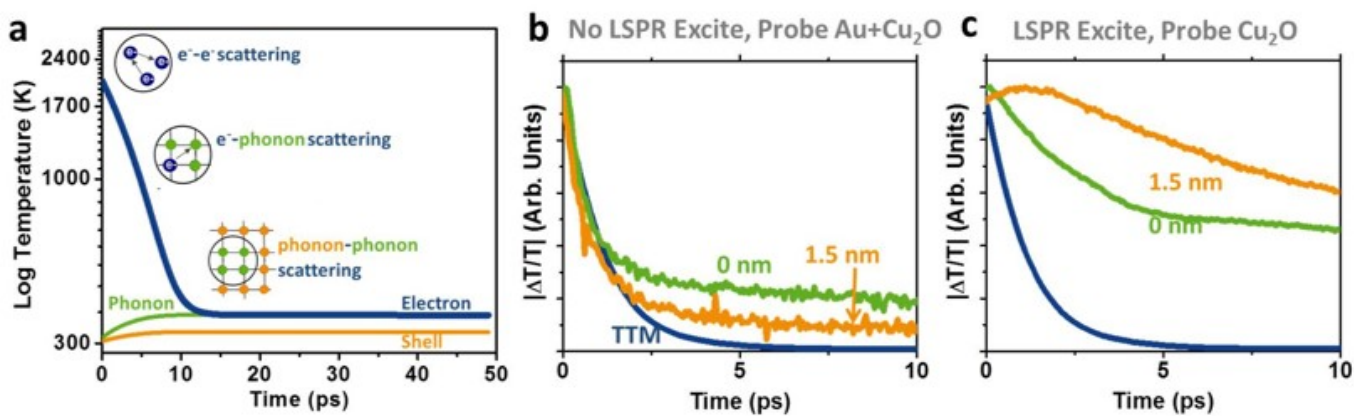

Figure S4.18. Two-temperature model for Au contribution to transient absorption signal. a, The two-temperature model (TTM) show that carrier relaxation in the Au nanoparticle proceeds through electron-electron scattering on a fs time scale, followed by electron-phonon scattering on a 1-10 ps time scale, and finally phonon-phonon scattering on a 100 ps or larger time scale. Changing the interface or $\mathrm{SiO}_{2}$ layer only changes the phonon-phonon scattering time, not the electron-phonon scattering due to the disparity in time scales. b, The TTM is seen to describe the data excellently when the Au nanoparticle and $\mathrm{Cu}_{2} \mathrm{O}$ are excited with a $400 \mathrm{~nm}$ pump pulse, not at the LSPR, and then probed with a $1400 \mathrm{~nm}$ wavelength which is primarily sensitive to carriers in the metal nanoparticle. c, The same model cannot describe the data when the LSPR is pumped and only the $\mathrm{Cu}_{2} \mathrm{O}$ is probed, proving the Au does not contribute to the signal. 

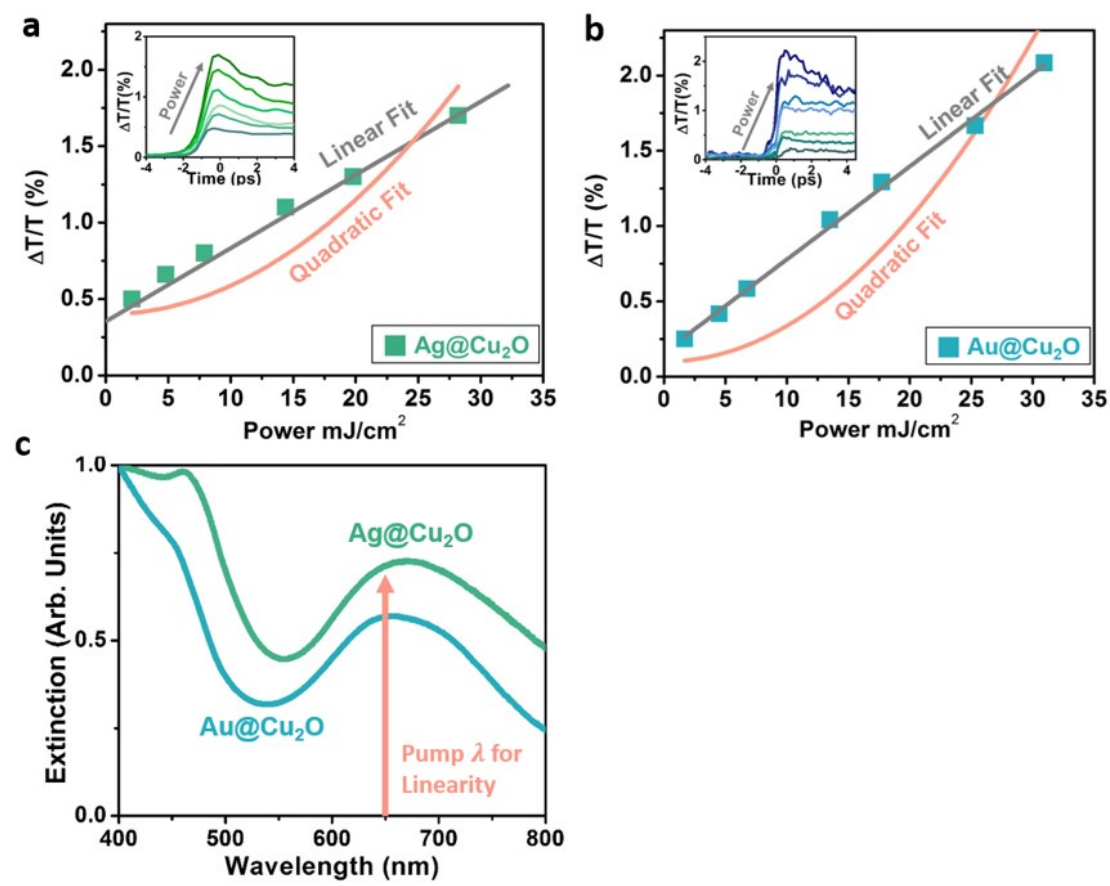

Figure S4.19. Power dependence of PIRET produced carriers probed by transient absorption. a, Data taken from $\mathrm{Ag} @ \mathrm{Cu}_{2} \mathrm{O}$ nanoparticles, inset shows transients for each power. Both a linear fit, suggestive of a one photon dipoledipole transfer, and a quadratic fit, suggestive of two photon absorption or second harmonic generation, are shown. b, Data taken from $\mathrm{Au} @ \mathrm{Cu}_{2} \mathrm{O}$ nanoparticles, inset shows transients for each power, linear and quadratic fits are shown for comparison. At high powers laser damage concerns prevent averaging, so the data is noisier. A power of $\sim 5 \mathrm{~mJ} / \mathrm{cm}^{2}$ was used in the main figures of the paper. c, UV-Vis absorption for $\mathrm{Ag} @ \mathrm{Cu}_{2} \mathrm{O}$ and $\mathrm{Au} @ \mathrm{Cu}_{2} \mathrm{O}$. The pump wavelength for parts (a) and (b) are shown, indicating the linearity was tested at peak plasmon absorption in both samples.
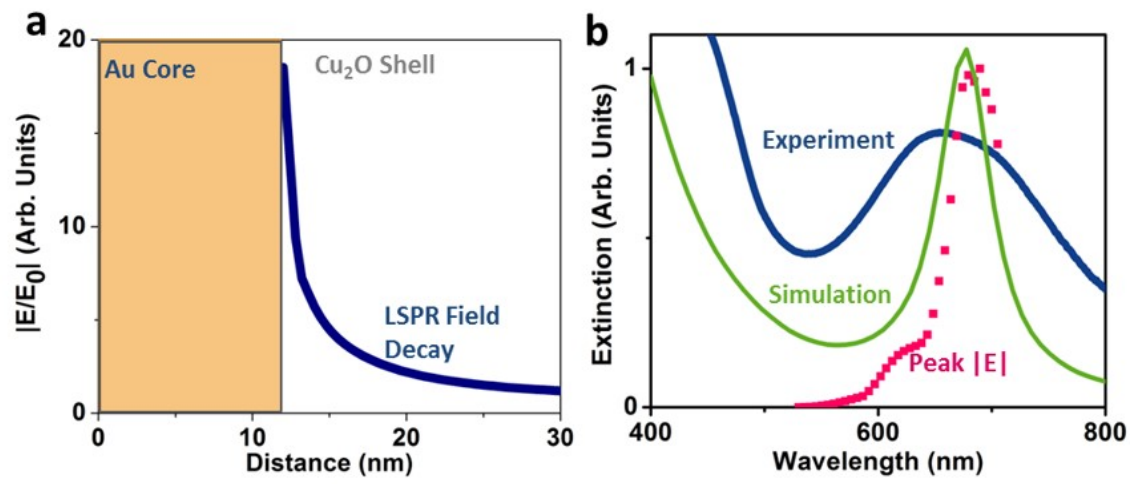

Figure S4.20. Discrete dipole approximation simulations for LSPR in the $\mathrm{Au} @ \mathrm{Cu}_{2} \mathrm{O}$ nanoparticle. a, The intense local field characteristic of LSPR decays outside the Au core as $1 / r^{3}$ which can be approximated by an exponential. $\mathbf{b}$, The simulated absorption for a single $\mathrm{Au} @ \mathrm{Cu}_{2} \mathrm{O}$ core shell nanoparticle agrees well with the center peak of the distribution measured experimentally. The peak of the electric field enhancement is slightly redshifted from the peak absorption. 

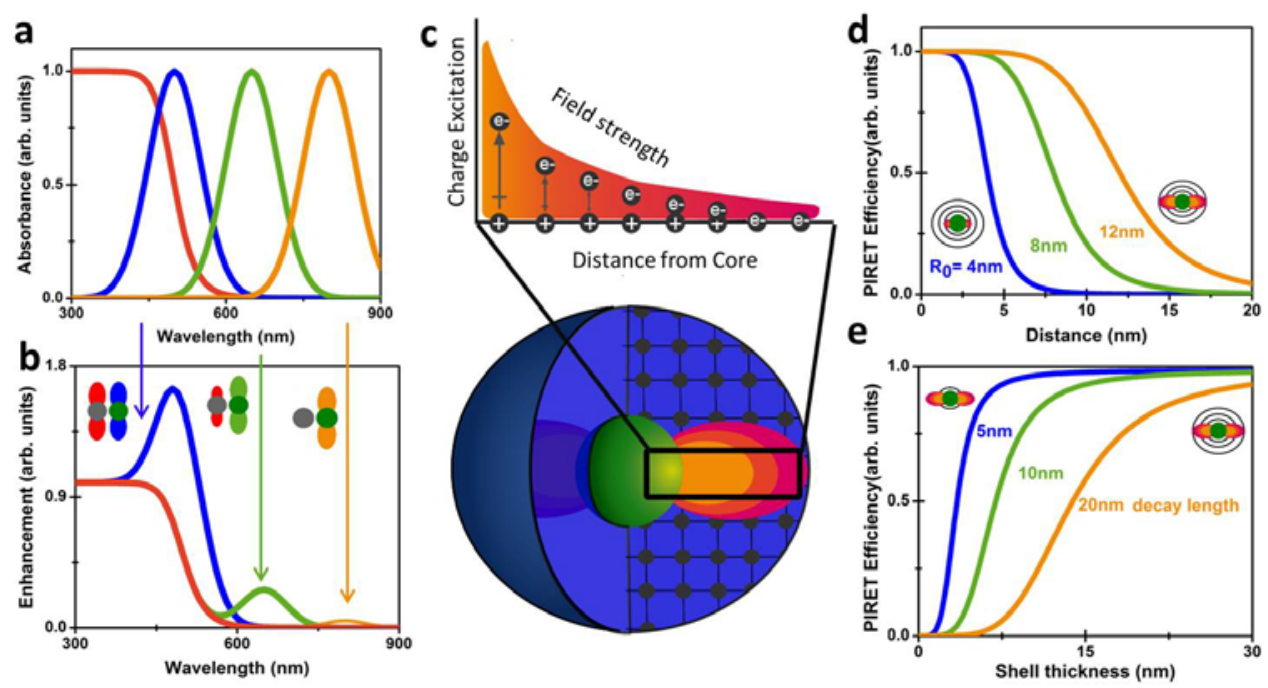

Figure S4.21. Coupling of plasmon and semiconductor through PIRET. a, The efficiency of PIRET depends on the spectral overlap of the LSPR and semiconductor, creating (b) an enhancement that weakens with decreasing spectral overlap. c, The LSPR EM field varies spatially over the extent of the semiconductor, varying the PIRET energy transfer efficiency with (d) the distance from the LSPR to the semiconductor and (e) the thickness of the semiconductor shell. In (e) the coupling is modified by a term representative of the LSPR decay into the semiconductor, see Fig. S4.20a, making

$$
R_{0}{ }^{\prime}=R_{0}\left[1-\exp \left(-\frac{d_{\text {semi }}}{\delta_{\text {LSPR }}}\right)\right] \text {. }
$$

This approximation is the same as ignoring the spatial variation of the LSPR field in the $\mathrm{Cu}_{2} \mathrm{O}$ shell, but limiting the magnitude of the coupling if the $\mathrm{Cu}_{2} \mathrm{O}$ shell thickness $d_{\text {semi }}$ is spatially smaller than the LSPR field decay $\delta_{L S P R}$ or

vice versa. The phenomenological model is validated in that it reproduces the trend of energy transfer being maximized when the semiconductor shell exceeds the LSPR field, and the exponential form is similar to that of the plasmon field decay. The $\mathrm{Cu}_{2} \mathrm{O}$ shell is kept greater than $30 \mathrm{~nm}$ throughout the paper to avoid incomplete coupling.

The non-uniform field may still lead to the excitation of dark modes or other deviations from PIRET theory as presented.
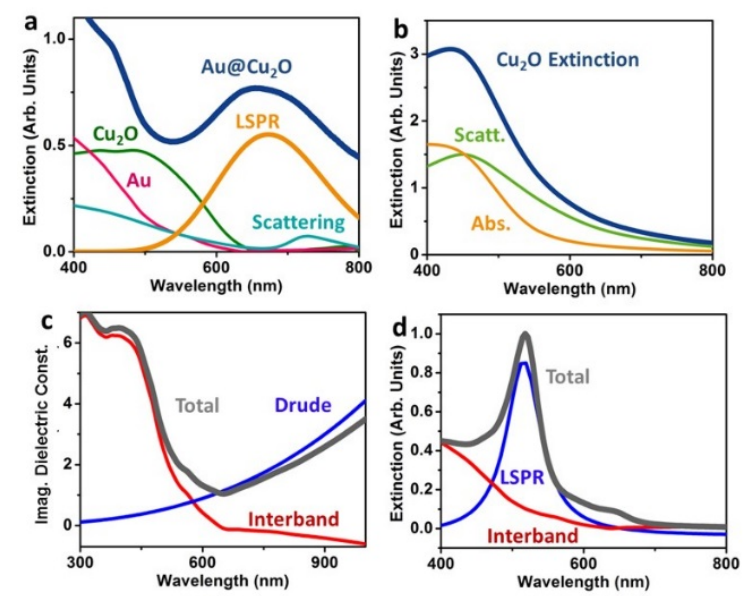

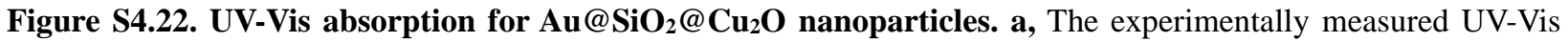
absorption is a combination of scattering, $\mathrm{Cu}_{2} \mathrm{O}$ band-edge absorption, interband transitions in $\mathrm{Au}$, and the LSPR peak. 
b, the $\mathrm{Cu}_{2} \mathrm{O}$ absorption itself includes both scattering and absorption components, shifting the apparent band-edge. c, The dielectric of gold can be split into both free electron Drude contributions and interband transitions, which lead to (d) the LSPR position and a separate absorption, respectively.
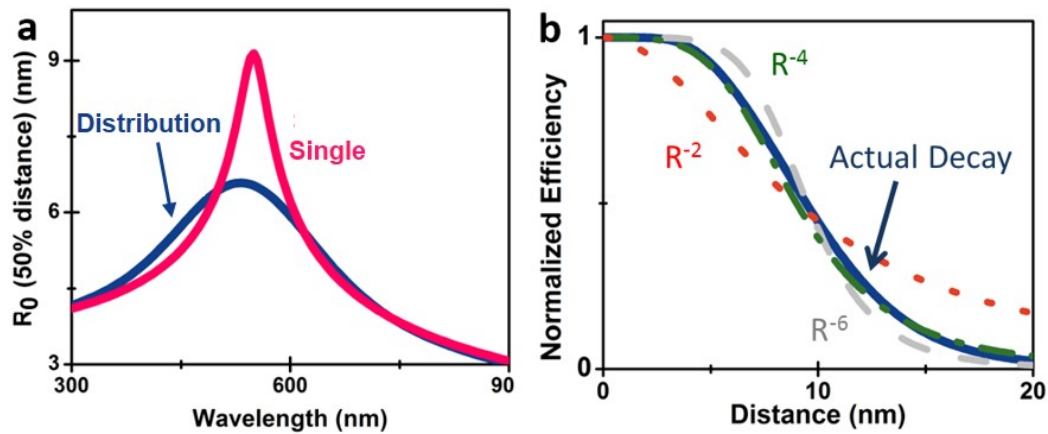

Figure S4.23. PIRET theory for a distribution of LSPR peaks. a, The average value of $R_{o}$ varies with excitation wavelength for both single oscillators and an inhomogeneously broadened distribution of oscillators centered at the same wavelength. The distribution is comprised of oscillators with linewidths comparable to a single plasmon resonance. The distribution's average $R_{o}$ is offset towards the overlap with the semiconductor. b, The excitation wavelength dependence of $R_{o}$ distorts the distance dependence efficiency from $1 / r^{6}$ to $1 / r^{4}$ for excitation wavelengths close to the semiconductor/LSPR overlap.

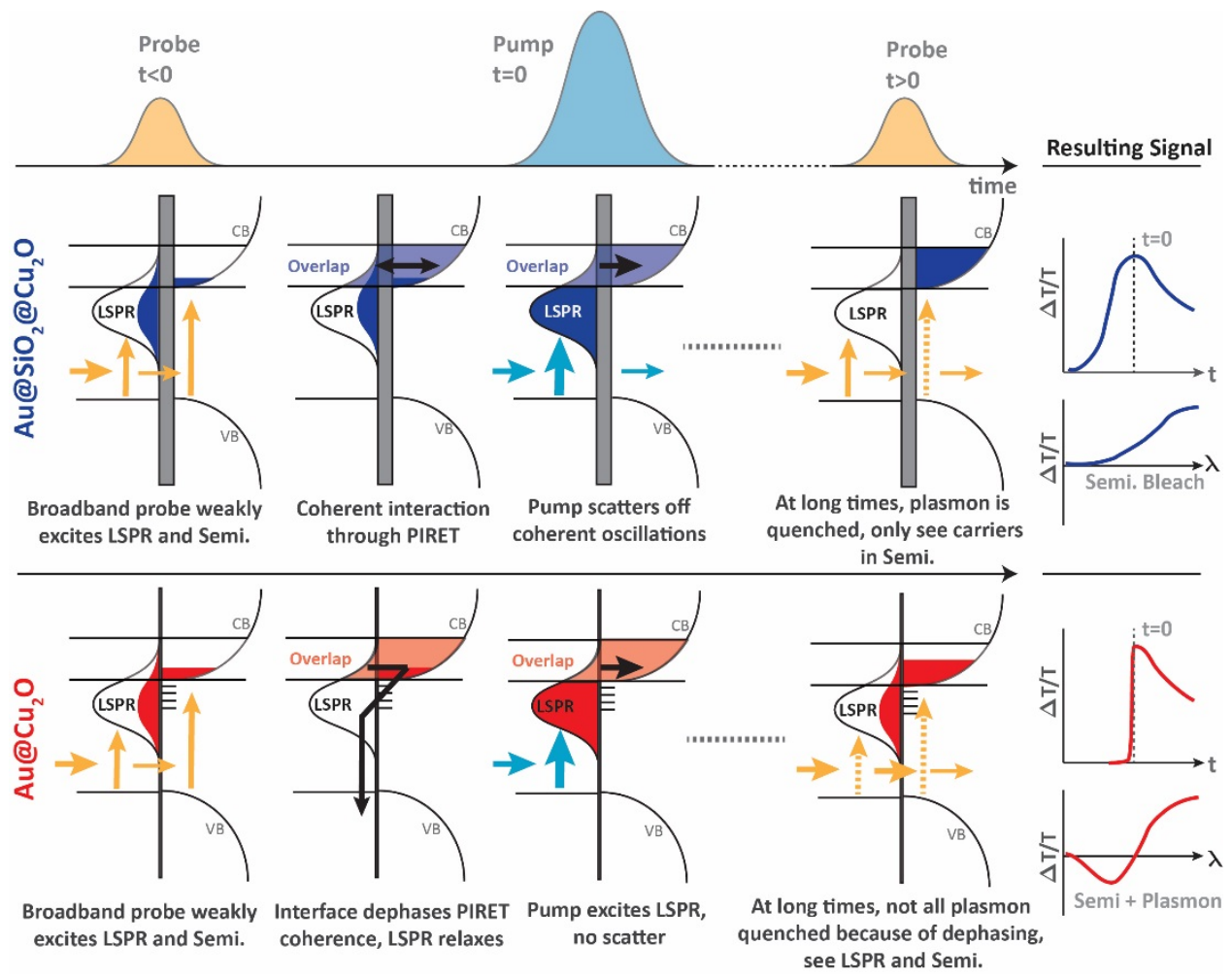

Figure S4.24. Explanation of coherence and measured signals in transient absorption. Before time $=0$ the broadband probe can set up a coherence between the $\mathrm{Cu}_{2} \mathrm{O}$ and plasmon since both are weakly excited. Due to the strong dipoledipole coupling, the two systems are excited in a superposition, or coherent state. If this state lasts long enough, as in $\mathrm{Au} @ \mathrm{SiO}_{2} @ \mathrm{Cu}_{2} \mathrm{O}$, the pump will scatter off the coherence before time=0, leading to oscillations in the broadband probe and a long rise time. At time $=0$ the strong pump fully excites the plasmon, making the probe excited changes negligible. The plasmon can then transfer its energy to the $\mathrm{Cu}_{2} \mathrm{O}$, quenching the transient absorption signal of the plasmon and creating a band-edge bleach in the $\mathrm{Cu}_{2} \mathrm{O}$. In the $\mathrm{Au} @ \mathrm{Cu}_{2} \mathrm{O}$, the plasmonic hot electron transfer dampens the coherence quickly, resulting in no scatter of the pump off the probe before time $=0$. At time $=0$, the pump excites 
the plasmon, resulting in partial transfer by PIRET before the plasmon decoheres because of the interfacial charge transfer. Since the dephasing occurs before all carriers can transfer by PIRET, the plasmon transient absorption signal still exists, in addition to the bleach from carriers transferred to the $\mathrm{Cu}_{2} \mathrm{O}$.

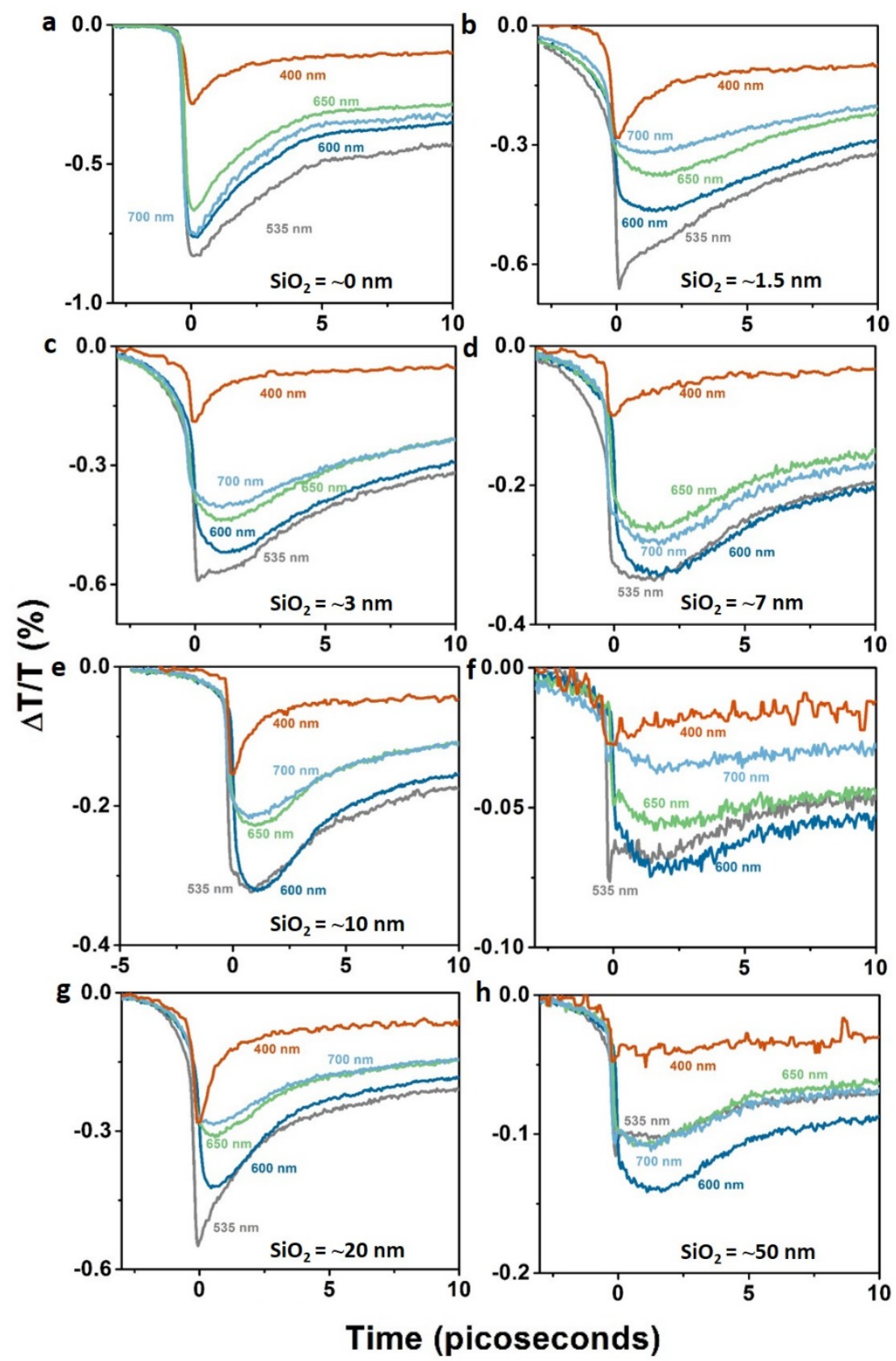

Figure S4.25. Raw data for all relative enhancement calculations. The raw data as labelled is shown for each excitation wavelength and distance. Please note differences in power and spot size are not yet corrected. For presentation purposes, a light averaging filter was used on some of the lower signal data, however all calculations and analysis were done before filtering throughout the paper unless noted otherwise. 


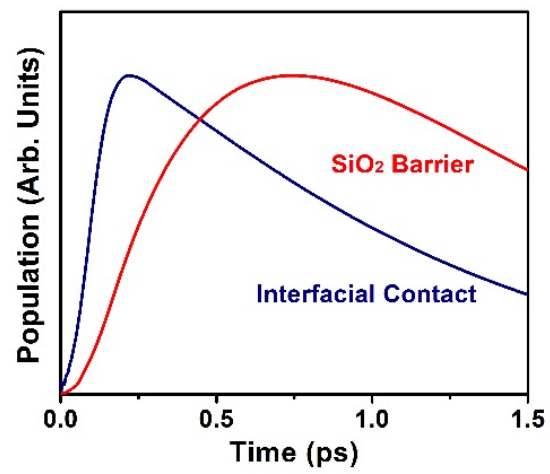

Figure S4.26. Effect of interface on transient population creation. Excitation of the plasmon with the $\mathrm{SiO}_{2}$ barrier in place by a 100 fs pulse ( $\tau_{\text {dephasing }}=5 f s, \tau_{\text {population }}=1 \mathrm{ps}$ ) led to a long rise time consistent with the experimental transients. Reduction of the dephasing and population time by contact $\left(\tau_{\text {dephasing }}=1 \mathrm{fs}, \tau_{\text {population }}=0.5 \mathrm{ps}\right)$ led to a sharper rise time and decay, also consistent with experiment. The other parameters for the model are identical to those outlined at the beginning of the SI.

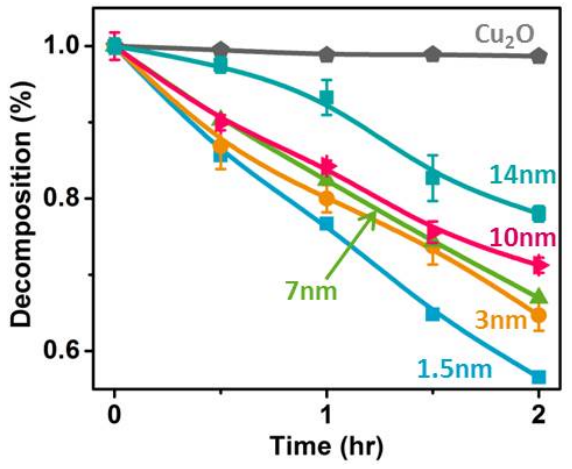

Fig. S4.27. Enhancement of photocatalysis by PIRET. Comparison of the PIRET enhancement verse $\mathrm{SiO}_{2} \mathrm{spacer}$ layer thickness for photocatalysis using visible excitation. The $1.5 \mathrm{~nm} \mathrm{SiO} 2$ spacer layer had the largest increase in photocatalytic activity compared to $\mathrm{Cu}_{2} \mathrm{O}$ alone for below band-edge excitation, with the decreasing rate resembling the measured distance dependence in transient absorption.

\begin{tabular}{|c|c|c|c|c|c|c|c|}
\hline $\begin{array}{l}\text { Thickness } \\
\mathrm{SiO}_{2}(\mathrm{~nm})\end{array}$ & $\begin{array}{l}\text { Lifetime } \\
\tau_{1}(\mathrm{~s})\end{array}$ & $\tau_{2}(\mathbf{s})$ & $\tau_{3}(\mathbf{s})$ & $\begin{array}{l}\text { Amplitud } \\
\mathbf{A}_{1}\end{array}$ & $\mathbf{A}_{2}$ & $\mathbf{A}_{3}$ & $\begin{array}{l}\text { Amplitude } \\
\text { Average (s) }\end{array}$ \\
\hline $\mathbf{0}$ & 1.52E-09 & 4.85E-11 & $1.80 \mathrm{E}-08$ & 2.21E-02 & 1.67 & 7.33E-05 & 6.85E-11 \\
\hline 1.5 & 2.03E-09 & $1.10 \mathrm{E}-12$ & 1.73E-08 & 4.33E-03 & $4.67 \mathrm{E}+21$ & $6.31 \mathrm{E}-05$ & $1.10 \mathrm{E}-12$ \\
\hline 3 & 1.45E-09 & $4.24 \mathrm{E}-11$ & 3.35E-08 & 0.0127 & 2.36 & 1.98E-05 & $5.02 \mathrm{E}-11$ \\
\hline 7 & $1.58 \mathrm{E}-09$ & $2.51 \mathrm{E}-11$ & $1.46 \mathrm{E}-08$ & 9.94E-03 & 7.27 & 8.10E-05 & $2.74 \mathrm{E}-11$ \\
\hline 10 & 1.52E-09 & $1.10 \mathrm{E}-10$ & 2.15E-08 & 2.40E-02 & 0.506 & 4.63E-05 & 1.76E-10 \\
\hline 14 & 1.55E-09 & $1.18 \mathrm{E}-10$ & $1.31 \mathrm{E}-08$ & $1.74 \mathrm{E}-02$ & 0.505 & $1.10 \mathrm{E}-04$ & $1.68 \mathrm{E}-10$ \\
\hline 20 & 2.05E-09 & $5.04 \mathrm{E}-10$ & $1.23 \mathrm{E}-08$ & 1.64E-02 & 0.131 & 2.23E-04 & 6.95E-10 \\
\hline 50 & 1.78E-09 & $4.56 \mathrm{E}-10$ & $1.88 \mathrm{E}-08$ & 2.00E-02 & 0.133 & $9.90 \mathrm{E}-05$ & $6.40 \mathrm{E}-10$ \\
\hline
\end{tabular}

Table 4.1. Fitting coefficients for time resolved fluorescence (TRF) data. The TRF decays required a triexponential fit convoluted with the instrument response function. The amplitude averaged lifetime, $\tau_{\text {avg }}=\sum \tau_{i} A_{i} / \sum A_{i}$ was used to determine the TRF quenching versus $\mathrm{SiO}_{2}$ spacer layer thickness. At $1.5 \mathrm{~nm}$ the amplitude averaged lifetime approached the instrument response function, indicating near complete TRF quenching on the time scales measurable. 


\subsection{Efficiency of PIRET}

Section 4.2 and 4.3 showed that PIRET is capable of creating more carriers in the band-edge region of the semiconductor than light absorption in the semiconductor at energies above the band gap. This was seen to translate into an increase in photocatalysis. However, dye degradation is less energetically demanding than water splitting or other solar-to-fuel processes. To test if PIRET was applicable to extending light absorption in the case of water splitting, PIRET was engineered into a semiconductor heterostructure similar to the test case in Section 3.3 for hot carrier transfer. Perovskite lanthanum titanium oxide $\left(\mathrm{La}_{2} \mathrm{Ti}_{2} \mathrm{O}_{7}\right)$ was nitrogen doped to extend the band gap to $\sim 500 \mathrm{~nm}$ and reduced graphene oxide (rGO) was added as a charge extraction layer along with $\mathrm{Pt}$ as a co-catalyst. ${ }^{77} \mathrm{Au}$ nanoparticles were then added to the composite for the dual roles of extending light absorption and improving charge extraction. The methods and results are outlined in Reference 77, but in brief, the combination of graphene oxide and Au nanoparticles was found to increase the hydrogen production rate from $30 \frac{\mathrm{umol}}{\mathrm{g} * h}$ to $150 \frac{\mathrm{umol}}{\mathrm{g} * h}$.

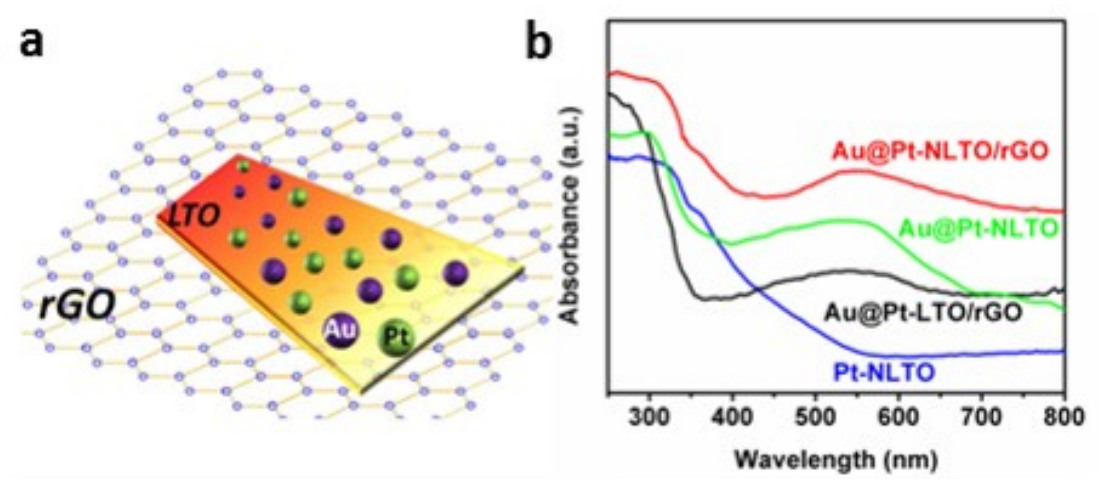

Figure 4.28. a, The Au@Pt-NLTO/rGO composite and b, UV-Visible spectra of the four samples. Reproduced from Reference 77.

To separate the effects of PIRET and charge extraction on the enhancement in hydrogen generation, the IPCE was measured as outlined in Reference 77 and shown in Figure 4.28. As discussed in the previous two Sections, plasmonic energy can transfer from the metal to semiconductor either via DET or PIRET process, inducing the charge separation in the semiconductor. In DET, the spectral enhancement follows the absorption of the plasmon, with a strength dependent on band alignment. In PIRET, the spectral enhancement and strength of transfer both follow the spectral overlap.

For the Au@Pt-LTO/rGO composite, the plasmonic Au nanoparticle can absorb light around $550 \mathrm{~nm}$. However, its IPCE at around $550 \mathrm{~nm}$ was zero (Figure 4.29a), which given that no spectral overlap was present for PIRET, means DET was weak. In the Au@Pt-NLTO/rGO composite, the absorption band of Ndoped LTO was overlapped with the LSPR band of Au nanoparticle (Figure 4.29d), leading to IPCE enhancement (Figure 4.29a). Since the photoconversion enhancement followed the spectral overlap and not the absorption of the plasmon, the dominant plasmonic energy transfer mechanism was most likely PIRET instead of DET. The same trend was seen in LTO and NLTO without the Pt and rGO, confirming these materials did not affect the plasmonic energy transfer or otherwise extend the photoconversion range. The lack of DET may be due to a large Schottky barrier present at the interface, or poor charge transfer kinetics from the plasmon to the LTO due to the ionic conductivity of LTO. PIRET is a nonradiative energy transfer mechanism, and therefore charge separation is not affected by the poor ionic conductivity, with the charge carriers being excited identically to under incident illumination. 

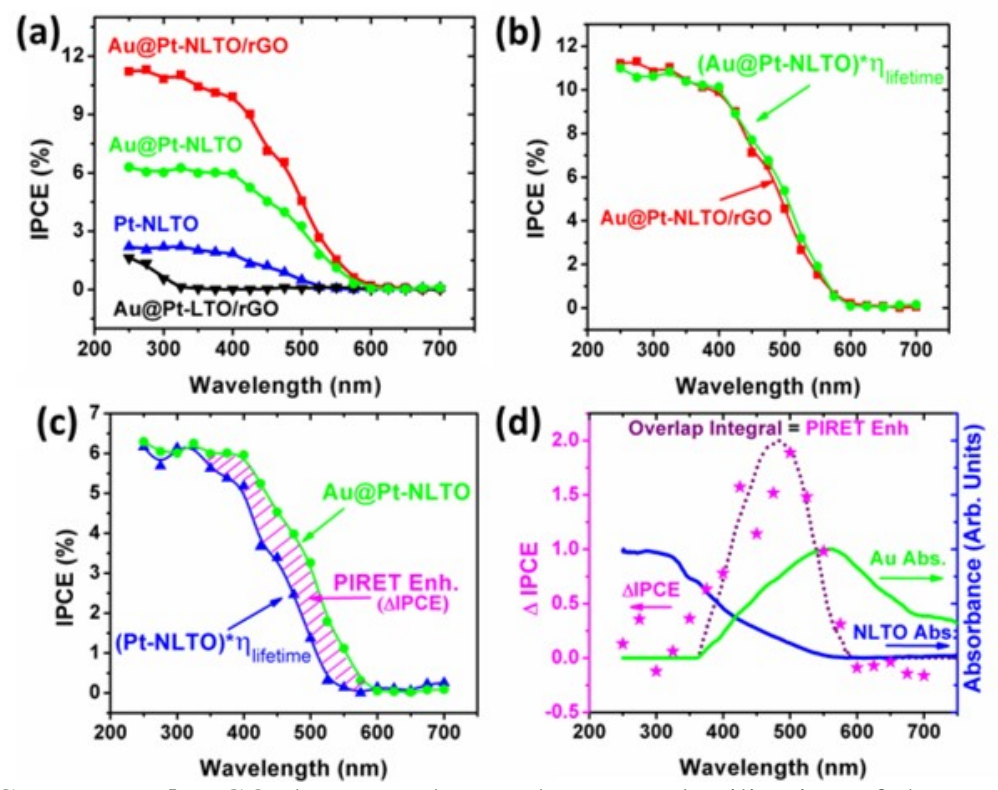

Figure 4.29. a, The IPCE spectra. b, rGO does not change the spectral utilization of the NLTO, and acts only to increase the IPCE by a constant factor $\eta_{\text {lifetime. }}$ c, Plasmonic energy transfer can increase the spectral utilization of the NLTO in addition to the factor $\eta_{\text {lifetime }}$, as shown by the hatched region. $\mathbf{d}$, The increase in the spectral utilization in the IPCE is seen to be proportional to the overlap integral between the Au and NLTO, proving the enhancement is from PIRET. Reproduced from Reference 77.

The overall hydrogen generation rate or photocurrent is proportional to the number of photo-generated charge carriers $(\alpha(h v))$ times the efficiency of carrier extraction ( $\left.\eta_{\text {lifetime}}\right)$, which is determined by the charge recombination, migration, and surface kinetics

$$
\text { Yield } \alpha(h v) \cdot \eta_{\text {lifetim }}
$$

The presence of rGO and the Fermi level equilibration effect of Au nanoparticles are expected to enhance the charge carrier extraction ( $\eta_{\text {lifetime}}$ ) not the number of photo-generated charge carriers $(\alpha(h v))$. Therefore, the IPCE enhancement from these effects is independent of the wavelength of incident light. In other words, the IPCE enhancement can be determined via multiplying by a constant factor. This behavior is seen by comparing the IPCE before and after the addition of rGO and Au nanoparticles (Figure 4.29b and4.29c). When the $\mathrm{Au}$ is added to the Pt-NLTO, it can increase the charge extraction by Fermi level equilibration and charge trapping. ${ }^{78-83}$ These enhancements only affect the lifetime of photo-generated charge carriers, and not the carrier generation versus the wavelength. Hence the IPCE of Au@Pt-NLTO above the band edge is equal to that of Pt-NLTO times the increase in $\eta_{\text {lifetime }}$ (Figure 4.29c), which is equal to $\sim 2.8$ times. Similarly, when rGO is added to the Au@Pt-NLTO, the charge extraction further increases by a factor of 1.8 (Figure 4.29b) although no spectral extension is seen for the addition of rGO into the undoped Au@PtLTO. The combination of Au and rGO increases the charge extraction efficiency by $~ 5$ times that of the PtNLTO alone. The constant increase also rules out a scattering enhancement by rGO, which would also enhance above the band edge but depend on wavelength.

An increase in the charge extraction efficiency completely describes the IPCE enhancement by the presence of rGO (Figure 4.29b). However, an increase in charge extraction efficiency only partially describes the IPCE enhancement by the presence of Au nanoparticles (Figure 4.29c). The remaining 
increase in spectral conversion when comparing the plasmonic sample with the non-plasmonic sample is shown by the hatched section in Figure 4.29c for Au@Pt-NLTO verse Pt-NLTO and for Au@Pt-NLTO/rGO verse Pt-NLTO in Figure 4.30. This enhancement was due to a PIRET-induced increase in the number of photo-generated charge carriers $(\alpha(h v))$ verse the wavelength of incident light. Figure 4.29d shows the extracted spectral enhancement of the IPCE, represented as

$$
\Delta \alpha(h v) \sim \triangle I P C E=I P C E_{\text {Au@NLTO }}-I P C E_{\text {NLTO }} .
$$

The enhancement $\Delta \alpha_{\text {PIRET }}(h v) / \alpha_{0}(h v)$ will be proportional to the overlap integral, which is itself proportional to the overlap of the absorption band of NLTO with the LSPR band of Au nanoparticles

$$
\Delta \alpha(h v) \sim \int \sigma_{N L T O}(\lambda) \cdot F_{A u}(\lambda) \cdot \lambda^{4} d \lambda,
$$

where $\sigma_{N L T O}(\lambda)$ is the absorption cross section of the NLTO, and the emission of the plasmon $F_{A u}(\lambda)$ can be approximated as the absorption cross section $\sigma_{A u}(\lambda)$ of the plasmon. The absorption cross section of the plasmon is extracted from Figure 4.28d by subtracting the absorption of the Pt-NLTO from the Au@PtNLTO sample, and then fitting the LSPR peak with a Gaussian to remove the absorption due to the interband transition in Au. If DET was present, the enhancement would only follow $\sigma_{A u}(\lambda)$ and not the overlap integral.
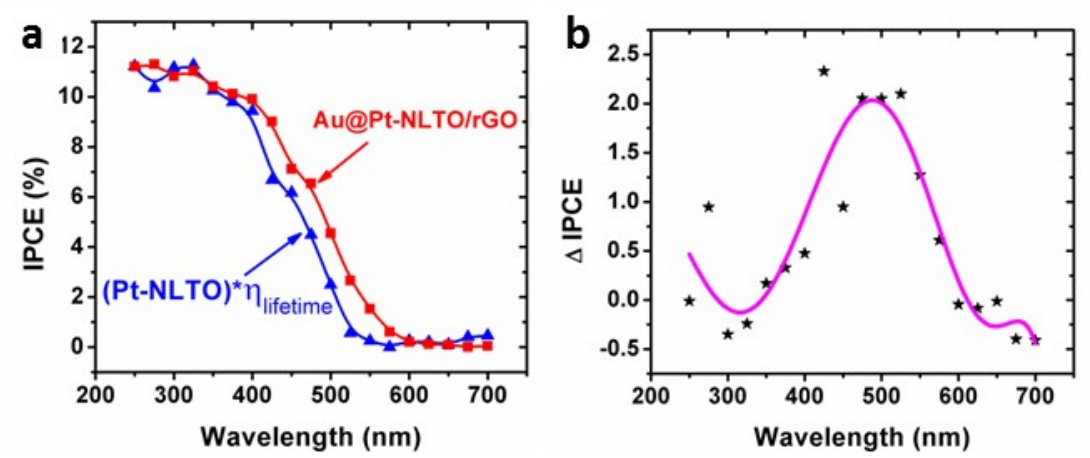

Figure 4.30. a, Increase in spectral utilization between the Pt-NLTO and Au@Pt-NLTO/rGO samples. b, The increase in the IPCE is seen to be identical to that predicted for PIRET. This is expected as the addition of rGO only increases $\eta_{\text {lifetime }}$, and not the spectral utilization. Therefore the plasmoni energy transfer mechanism is PIRET for both Au@PtNLTO and Au@Pt-NLTO/rGO samples. Reproduced from Reference 77.

The resulting overlap integral was calculated as shown in Figure 4.29d, and is seen to fit the spectral enhancement of the IPCE excellently. This further proves that PIRET is the underlying mechanism of carrier creation enhancement, and that the interaction of the doping and plasmonics extends the spectral utilization of the semiconductor. The total enhancement over the measured solar spectrum,

$$
\frac{\Delta \alpha_{\text {PIRET }}(h v)}{\alpha_{0}(h v)}=\int \Delta I P C E_{\text {PIRET }}(h v) / \int I P C E_{\text {NLTO }}(h v),
$$

can be calculated by integrating the corresponding IPCE spectrum. This gives the enhancement as $~ 60 \%$ for the Au@Pt-NLTO (Figure 4.29d) and 68 \% for the Au@Pt-NLTO/rGO (Figure 4.30). Further, using the measured absorption, the maximum possible enhancement by PIRET can be estimated by calculating the increase in the IPCE if all the energy absorbed by the plasmonic Au was transferred to the NLTO,

$$
\frac{\Delta \alpha_{\text {total }}(h v)}{\alpha_{0}(h v)}=\left(\int \Delta \sigma_{\text {Au }} * I_{\text {SUN }}-\int \sigma_{\text {NLTO }} * I_{\text {SUN }}\right) / \int \sigma_{\text {NLTO }} * I_{\text {SUN }} \text {, }
$$

where $I_{\text {sun }}$ is the AM1.5G spectrum. If the measured enhancement is divided by this maximum value, calculated to be $\sim 100 \%$ of the NLTO IPCE, the value is equal to the PIRET efficiency, or how well PIRET transfers absorbed energy in the Au to the NLTO. Using this procedure, the PIRET transfer efficiency was 
found to be 60 \% for the Au@Pt-NLTO. This implies that 60 \% of the solar energy absorbed by the LSPR is being transferred into the NLTO and converted into a photocurrent.

\subsection{Chapter Summary}

In this Chapter the plasmon-induced resonant energy transfer mechanism was shown to be the route through which the plasmon's near field can enhance photoconversion in a semiconductor. The PIRET mechanism was examined in-depth, showing that the dipole-dipole coupling is unlike FRET because the plasmon's coherence must be taken into account. Interestingly, this means that interface damping can highly affect the efficiency of PIRET, and that the most efficient plasmonic enhancement often occurs with no contact between metal and semiconductor. PIRET was shown to be highly efficient when dephasing was taken into account, creating as many carriers in a semiconductor near the band edge as the same flux of UV light could above the band edge.

In the final section it was shown that, unlike in the case of adding Au nanoparticles in Chapter 3, PIRET is able to create an enhancement for solar water splitting that is equal to the effects of the metal nanoparticle on charge separation, leading to a large increase in solar energy conversion efficiency. This high efficiency for PIRET is consistent with Section 4.2 and 4.3 as well as further experiments on $\mathrm{Ag} @ \mathrm{Cu}_{2} \mathrm{O}$, which also had an equal near-band edge to above band edge photocatalysis efficiency enhancement. ${ }^{43}$ Chapters 2 through 4 therefore show that, just as in plasmon-enhanced fluorescence and Raman scattering, the plasmon's near field leads to the largest and most consistent enhancements. ${ }^{1}$ These results indicate that the plasmon's near field is often the best route for solar energy enhancement in both light-trapping and spectralrange extending situations.

\subsection{References}

1. Li, M.; Cushing, S. K.; Wu, N. Analyst 2014, 140, 386-406.

2. Linic, S.; Christopher, P.; Ingram, D. B. Nat. Mater. 2011, 10, 911-921.

3. Warren, S. C.; Thimsen, E. Energy Env. Sci 2012, 5, 5133-5146.

4. Thimsen, E.; Le Formal, F.; Grätzel, M.; Warren, S. C. Nano Lett. 2011, 11, 35-43.

5. Ingram, D. B.; Linic, S. J. Am. Chem. Soc. 2011, 133, 5202-5205.

6. Thomann, I.; Pinaud, B. A.; Chen, Z.; Clemens, B. M.; Jaramillo, T. F.; Brongersma, M. L. Nano Lett. 2011, 11, 3440-3446.

7. Standridge, S. D.; Schatz, G. C.; Hupp, J. T. J. Am. Chem. Soc. 2009, 131, 8407-8409.

8. Awazu, K.; Fujimaki, M.; Rockstuhl, C.; Tominaga, J.; Murakami, H.; Ohki, Y.; Yoshida, N.; Watanabe, T. A. J. Am. Chem. Soc. 2008, 130, 1676-1680.

9. Liu, Z.; Hou, W.; Pavaskar, P.; Aykol, M.; Cronin, S. B. Nano Lett. 2011, 11, 1111-1116.

10. Zhang, X. et al. Nanoscale 2013, 5, 3359-3366.

11. Förster, T. Ann. Phys. (Berlin) 1948, 437, 55-75.

12. Lessard-Viger, M.; Rioux, M.; Rainville, L. \& Boudreau, D. Nano Lett 2009, 9, 3066-3071.

13. Lakowicz, J.R. Principles of Fluorescence Spectroscopy; Springer Academic: New York, 2006.

14. Lunz, M. et al. Nano Lett. 2011, 11, 3341-3345.

15. Hu, C.; Peng, T.; Hu, X.; Nie, Y.; Zhou, X.; Qu, J.; He, H. J. Am. Chem. Soc. 2010, 132, 857-862.

16. Christopher, P.; Ingram, D. B.; Linic, S. J. Phys. Chem. C 2010, 114, 9173-9177.

17. Furube, A.; Du, L.; Hara, K.; Katoh, R.; Tachiya, M. J. Am. Chem. Soc. 2007, 129, 14852-14853.

18. Tian, Y.; Tatsuma, T. Chem. Commun. 2004, 1810. 
19. Kowalska, E.; Mahaney, O.; Abe, R.; Ohtani, B. Phys. Chem. Chem. Phys. 2010, 12, 2344.

20. Mubeen, S.; Hernandez-Sosa, G.; Moses, D.; Lee. J.; Moskovits, M. Nano Lett. 2011, 11, 55485552.

21. Cushing, S. K.; Li, J.; Meng, F.; Senty, T. R.; Suri, S.; Zhi, M.; Li, M.; Bristow, A. D.; Wu, N. J. Am. Chem. Soc. 2012, 134, 15033-15041.

22. Jain, P. K.; Huang, X.; EI-Sayed, I. H.; EI-Sayed, M. A. Acc. Chem. Res. 2008, 41, 1578-1586.

23. Frens, G. Nature Phys. Sci. 1973, 241, 20-22.

24. Liz-Marzan, L. M.; Giersig, M.; Mulvaney, P. Langmuir 1996, 12, 4329-4335.

25. Draine, B.T.; Flatau, P. J. J. Opt. Soc. Am. 1994, A11, 1491-1499.

26. Palik, E. D. Handbook of Optical Constants of Solids; Academic Press: Boston, 1985.

27. Rycenga, M.; Cobley, C. M.; Zeng, J.; Li, W.; Moran, C. H.;. Zhang, Q.; Qin, D.;Xia, Y. Chem. Rev. 2011, 111, 3669-3712.

28. Dressel, M. and Gruner, G. Electrodynamics of Solids; Cambridge University Press: Cambridge, 2002.

29. Rzazewski, K.; Boyd, R. W. Journal of Modern Optics 2004, 51, 1137-1147.

30. Kosuda, K. M.; Bingham, J. M.; Wustholz, K. L.; Van Duyne, R. Comprehensive Nanoscience and Technology 2011, 3, 263-301.

31. Andrews, D. L. Chem. Phys. 1989, 139, 195-201.

32. Jacak, J.; Krasnyj, J.; Jacak, W.; Gonczarek, R.; Chepok, A.; Jacak, L., Phys. Rev. B. 2010, 82, 035418.

33. Jung, J.; Trolle, M. L.; Pederson, K.; and Pederson, T. G. Phys. Rev. B. 2011, 84, 165447.

34. Shockley, W.; Read, W.T. Phys. Rev. 87, 835-842 (1952).

35. Bolton, J.; Strickler, S.; Connolly, J. Nature 1985, 316, 495-500.

36. De Vos, A. J. Phys. D: Appl. Phys. 1980, 13, 839-846.

37. Ross, R.T.; Nozik, A.J. J. Appl. Phys. 1982, 53, 3813-3818.

38. Hanna, M.C.; Nozik, A.J. J. Appl. Phys. 2006, 100, 075410.

39. Vasa, P. et al. Nature Photon. 2013, 7, 128-132.

40. Dai, X. et al. Phys. Rev. Lett. 2012, 108, 193201.

41. Engel, G.S. et al. Nature 2007, 446, 782-786.

42. Tabachnyk, M. et al. Nature Mater. 2014, 13, 1033-1038.

43. Li, J. et al. ACS Catal. 2013, 3, 47-51.

44. Li, J. et al. Nature Commun. 2013, 4, 2651.

45. Meng, F.; Cushing, S.K.; Li, J.; Hao, S.; Wu, N. ACS Catal. 2015, 5, 1949-1955.

46. Li, J.; Cushing, S. K.; Zheng, P.; Meng, F.; Chu, D.; Wu, N. Nat. Commun. 2013, 4, 2651.

47. Li, J.F. et al. Nature 2010, 464, 392-395.

48. Kuo, C.H.; Hua, T.; Huang, M.H.; J. Am. Chem. Soc. 2009, 131, 17871-17878.

49. Nitzan, A. Chemical Dynamics in Condensed Phases; Oxford University Press: New York, 2006.

50. Varada, G.V.; Agarwal, G.S. Phys. Rev. A 1992, 45, 6721-6729.

51. Sadeghi, S.M. Phys. Rev. A 2013, 88, 013831.

52. Zhang, W.; Govorov, A.O.; Bryant, G.W. Phys. Rev. Lett. 2004, 97, 146804.

53. Zayats, A.V.; Maier, S.A. Active Plasmonics and Tuneable Plasmonic Metamaterials; Wiley: New Jersey, 2013.

54. Artuso, R.D.; Bryant, G.W. Phys. Rev. B 2010, 82, 195419.

55. Manjavacas, A.; Nordlander, P.; Garcia de Abajo, F.J. ACS Nano 2013, 6, 1724-1731. 
56. Jang, S.; Cheng, Y.C.; Reichman, D.R.; Eaves, J.D. J. Chem. Phys. 2008, 129, 101104.

57. Becker, P.C. et al. Phys. Rev. Lett. 1988, 61, 1647-1649.

58. Rosencher, E. Optoelectronics; Cambridge University Press: New York, 2002.

59. Li, Y.; Zhao, K.; Sobhani, H.; Bao, K.; Nordlander, P. J. Phys. Chem. Lett. 2013, 4, 1352-1357.

60. Mauritz, O.; Goldoni, G.; Rossi, F.; Molinari, E. Phys. Rev. Lett. 1999, 82, 847-850.

61. Zhang, X. et al. ACS Nano 2012, 6, 9283-9290.

62. Zhang, X. et al. ACS Nano 2014, 8, 1273-1283.

63. Pinchuk, A.; Kreibig, U. New J. Phys. 2003, 5, 151.

64. Olsen, L.C.; Bohara, R.C.; Urie, M.W. Appl. Phys. Lett. 1979, 34, 47-49.

65. Palfrey, S.I.; Heinz, T.F. J. Opt. Soc. Am. B 1985, 2, 674-679.

66. Guenther, T. et al., Phys. Rev. Lett. 2002, 89, 057401.

67. Link, S.; El-Sayed, M.A. J. Phys. Chem. B 1999, 103, 8410-8426.

68. Falasconi, M. et al. Surf. Sci. 2001, 481, 105-112.

69. Kauranen, M.; Zayats, A. V. Nature Photon. 2012, 6, 737-748.

70. Peña-Rodríguez, O.; González Pérez, P. P.; Pal, U. Int. J. Spectrosc. 2011, 583743, 1-10.

71. Liu, X.; Atwater, M.; Wang, J.; Huo, Q. Colloids Surf., B 2007, 58, 3-7.

72. Moskovits, M. Nature Nanotechol. 2005, 10, 6-8.

73. Shah, J. Ultrafast Spectroscopy of Semiconductors and Semiconductor Nanostructures; Springer: New York, 1999.

74. Rashidi-Huyeh, M.; Palpant, B. J. Appl. Phys. 2005, 96, 4475.

75. Rashidi-Huyeh, M.; Volz, S.; Palpant, B. Phys. Rev. B 2008, 78, 125408.

76. Linnros, J. J. Appl. Phys. 1998, 84, 284.

77. Meng, F.; Cushing, S. K.; Li, J.; Hao, S.; Wu, N. ACS Catal. 2015, 5, 1949-1955.

78. Li, J. T.; Cushing, S. K.; Zheng, P.; Senty, T.; Meng, F. K.; Manivannan, A.; Wu, N. Q. J. Am. Chem. Soc. 2014, 136, 8438-8449.

79. Mubeen, S.; Hernandez-Sosa, G.; Moses, D.; Lee., J.; Moskovits, M. Nano Lett. 2011, 11, $5548-5552$.

80. Subramanian, V.; Wolf, E. E.; Kamat, P. V. J. Am. Chem. Soc. 2004, 126, 4943-4950.

81. Jakob, M.; Levanon, H.; Kamat, P. V. Nano Lett. 2003, 3, 353-357.

82. Rosseler, O.; Shankar, M. V.; Karkmaz-Le Du, M.; Schmidlin, L.; Keller, N.; Keller, V. J. J. Catal. 2010, 269, 179-190.

83. Tada, H.; Mitsui, T.; Kiyonaga, T.; Akita, T. Tanaka, K. Nat. Mat. 2006, 5, 782-786. 


\section{Chapter 5. Outlook: Dephasing, Control, and Maximum Efficiency}

\subsection{Introduction}

The ability of plasmonics to manipulate, absorb, and concentrate light to increase photoconversion both above and below the semiconductor band edge, as shown in Chapters 2-4, make it an obvious solution to the band gap and light absorption issues that limit current generation, cheap-to-implement photovoltaics and solar-to-chemical conversion. However, despite the potential shown, outside of use in backreflectors/light-trapping plasmonics and recent demonstrations involving PIRET, plasmonics rarely appears in the top performing solar energy conversion devices. This is not because plasmonics has little potential, but rather because the complex response of the plasmon to incident light and its sub-picosecond lifetime obscures the underlying enhancement mechanisms, making systematic application difficult. For example, in Chapters 2-4 the plasmon's different aspect were specifically isolated to discover how each interaction could occur. However, when a metal nanoparticle is in contact with a semiconductor, any of the three enhancements could exist to different degrees. The plasmon's response is not binary. When light is incident on the metal nanoparticle all three responses exist to some extent.

To gain traction into the problem, it can be realized that the balance between the plasmon's response to incident light, and thus each enhancement mechanisms strength, depends on how quickly the collective electron oscillations cease to be collective. This time scale is known as the dephasing time. The plasmon's dephasing, and thus its optical response and enhancement of the semiconductor, changes with the size, shape, and metal of the nanoparticle, as well as the metal-semiconductor heterostructure band alignment and geometry. This versatility is therefore both plasmonic's strength and its weakness. While the plasmon is easily tuned to where a semiconductor needs to absorb more light, it cannot be assumed that spectral matching and metal nanoparticle integration alone is sufficient for a large solar energy enhancement. As shown in Chapter 4, the plasmon's dephasing is key, with interfacial damping changing the plasmon's response and transfer efficiency drastically.

The collection of results from this thesis have revealed that in order to create high efficiency plasmonic solar energy devices, the plasmon must be viewed beyond its UV-Vis spectroscopic properties. In particular, the dephasing and metal-semiconductor interface/geometry appear to be foremost in optimizing the different enhancement routes. In the following sections it is therefore shown how the plasmonic enhancements are related to dephasing, how the solar energy conversion can be optimized for each enhancement mechanism, and finally how systematic design can be used to achieve this goal.

\subsection{Dephasing And Maximum Efficiency Of Plasmonic Enhancement}

Shockley and Queisser's (SQ) calculations ${ }^{1}$ revolutionized solar materials design by providing a framework to systematically improve photovoltaics and solar-to-chemical conversion by optimizing thermalization losses. The predicted maximum efficiencies are based on a thick-film limit in which all incident solar light is absorbed. Unfortunately, as discussed in Chapter 1, recombination losses necessitate thin films, negating the thick-film limit and preventing the predicted efficiencies from being obtained in practical structures. ${ }^{2}$ The energy required for water splitting is also incongruent with the ideal band gap for solar energy conversion, further constraining realizable efficiencies. ${ }^{3,4}$

Subsequent generations of solar design have proposed to increase efficiencies by further decreasing thermalization losses, ${ }^{5-10}$ but the issue of how to balance recombination and light absorption losses still 
exists. In this thesis it has been shown that plasmonic enhancement offers a different approach, focusing on increasing absorption in the semiconductor above and below the band gap instead of minimizing thermalization losses. When isolated, a metal can only weakly led to photoconversion, with most incident light converted to heat. The metal's plasmon, however, excels at trapping and manipulating light on a per volume basis with a dipole moment orders of magnitude larger than a semiconductor. ${ }^{11,12}$ Plasmonic enhancement can therefore allow thick-film limits to be achieved in thin films and nanostructures.

Chapters 2-4 have proven solar-energy conversion can be enhanced by plasmonics in three ways. To gain further insight into how these enhancements are linked, it is best to examine the progression of the plasmon after excitation. When light is resonant with the localized surface plasmon resonance (LSPR) frequency the conduction electrons of the metal oscillate collectively, concentrating the incident light in the near field. The energy stored in the near field can be re-radiated as scatter, or the collective electron oscillations can dephase, destroying the coherence of the plasmon and leaving a hot-electron distribution. ${ }^{13}$ Scattering from the plasmon can be used for light trapping, increasing the path length of the photon through the semiconductor and the probability for absorption. ${ }^{14-18}$ The plasmonic hot electrons can overcome an interfacial Schottky barrier with a semiconductor, creating excited carriers in the semiconductor but where the plasmon absorbs, similar to a photosensitizer. ${ }^{19-23}$ The plasmon's near field can non-radiatively excite carriers in the semiconductor through plasmon-induced resonance energy transfer (PIRET), enhancing photoconversion where the semiconductor absorbs weakly. ${ }^{24-28}$

The relative strengths of scattering, hot electrons, and PIRET are connected by the rate at which the collective electron oscillations cease, known as the dephasing time. For bulk Au and Ag, the reported dephasing time ranges from 10-30 fs, with damping increasing at energies above the interband transition threshold of 2.3 and $3.9 \mathrm{eV}$ respectively. ${ }^{13}$ Large metal nanoparticles $(R \sim 50 \mathrm{~nm})$ generally have plasmon frequencies below the interband threshold, and given that radiative damping increases with volume, scattering dominates the plasmon's response. ${ }^{29}$ As the size is reduced $(R \sim 15 \mathrm{~nm})$, the radiative efficiency drops and interband damping increases, with the near field dominating the optical response as well-known from surface enhanced Raman scattering (SERS). ${ }^{30}$ If the size is further reduced $(R \sim 3 \mathrm{~nm}$ ), high surface scattering rates cease the collective electron motion almost instantaneously, eliminating the resonance absorption peak, with incident light only heating the metal through hot carrier creation. ${ }^{31}$ The importance of the plasmon's dephasing in determining the efficiency of near-field versus hot electron enhancement was recently seen experimentally, ${ }^{25}$ with the degree of interface damping controlling whether energy absorbed by the plasmon was transferred non-radiatively to a semiconductor or converted to heat. The complex response of the plasmon makes optimization for a given semiconductor's weaknesses difficult, as depending on the size of the metal nanoparticle, the position of the plasmon peak, and the metalsemiconductor interface, ${ }^{26}$ any or all of the three enhancement pathways could be present.

Therefore, in this Section, ${ }^{35}$ a density matrix model ${ }^{25,32-35}$ is extended to include coupling between a plasmon and a semiconductor in SQ theory, relying on the plasmon's dephasing to simultaneously optimize the three enhancement pathways for photovoltaics (PV) and photo-electrochemical cells (PEC). For a single nanoparticle, scattering was found most efficient for dephasing times close to the bulk metal (20-30 fs, $R \sim 50 \mathrm{~nm}$ ), replicating larger metal nanoparticles, with the plasmon increasing absorption above the band edge at energies where thermalization losses were least. Hot electron production rates should increase for small metal nanoparticles ( $<3 \mathrm{fs}, R \sim 3 \mathrm{~nm}$ ), however, hot electron transfer was found optimal at dephasing times (3-10 fs, $R \sim 15 \mathrm{~nm}$ ) which balance increased hot carrier production against the decrease in absorption from linewidth broadening, and at plasmon energies well below the band gap of the semiconductor. PIRET was found most efficient when the plasmon's dephasing (5-10 fs, $R \sim 15 \mathrm{~nm}$ ) is similar to the semiconductor 
and the plasmon's energy overlaps the semiconductor's band edge. Overall, PIRET created the largest enhancement, almost double the PV and PEC efficiency versus the semiconductor alone. PIRET led to the highest enhancement because the plasmon's larger dipole moment allowed energy transfer at dephasing times which broadened the plasmon's linewidth to maximize spectral coverage.

\subsubsection{Theory and Model}

Density Matrix Model The density matrix model is explained in depth in the Supplementary Calculations section after the Discussion and Conclusions for this section. Briefly, the density matrix model was chosen to represent the plasmon since it allows the importance of the dephasing ${ }^{13,25}$ in the plasmon's short-lived, coherent response to be captured. The density matrix treats the plasmon statistically, capturing the average optical dynamics of the ensemble of electron oscillations, both when collective and when dephased relative to each other. ${ }^{25,32-35}$ The density matrix also allows dipole-dipole coupling and scattering to be treated in the coherent and incoherent limits. This is crucial as the collective dipole moment decreases to that of the individual interband and intraband transitions after the plasmon dephases, changing the dipole-dipole coupling strength.

However, the density matrix model does neglect the size-dependent scattering and absorption probabilities given by Mie theory, as well as the size-dependent distribution of interband and intraband hot electrons and holes. ${ }^{22}$ To overcome these omissions, the yield of scattering and hot electron processes are fixed at their theoretical upper limits, ignoring the details of generation, but capturing how the semiconductor can best be enhanced spectrally by these processes. The semiconductor itself is treated under the framework of balancing band gap versus thermalization losses as consistently used for guiding solar design. ${ }^{1-10}$ The maximum efficiency calculations in this paper are therefore intended to place an upper limit on what is possible using the different plasmonic enhancement mechanisms, show how the plasmon's response can be best tailored towards the different enhancement routes, and guide what size and shape nanoparticle could best achieve these goals.

In the density matrix, the diagonal elements represent the mean population of the statistical ensemble of oscillators, and the off-diagonal elements represent the induced polarization. The plasmon (Figure 5.1a) was modeled as an ensemble of dipole oscillators with a recombination time $T_{1}$ and a dephasing time $1 / T_{2}=1 /\left(2 T_{1}\right)+1 /\left(T_{2}^{*}\right)$. The factor of two times $T_{1}$ ensures the correct decay rate of the population and $T_{2}^{*}$ is pure dephasing. The natural linewidth broadening in the absorption and scattering cross sections is given by $T_{2}$. The parameters were transferred into the density matrix (Figure 5.1b) and solved using the quantum master equation. Further details of the density matrix model can be found in the Supplementary Information, and the coupled-dipole model was based on Reference 36.

The excited state population and the polarization represented the optical response of the plasmon, calculated by the expectation of the on and off-diagonal components of the density matrix respectively. The plasmon's evolution is also shown schematically in Figure 5.1d. When light first excites the collective electron oscillations, incident energy is concentrated in the strong local field. The plasmon is initially in a coherence with the incident field and an uncertainty existed in its population. The uncertainty leads to the plasmon's linewidth through the energy-time uncertainty principle. ${ }^{37}$ The collective nature of the plasmon leads to a dipole moment an order of magnitude larger than in a semiconductor, creating large resonance absorption (population, Figure 5.1c), or under collective de-excitation, resonance scattering cross sections (polarization, Figure 5.1c). 


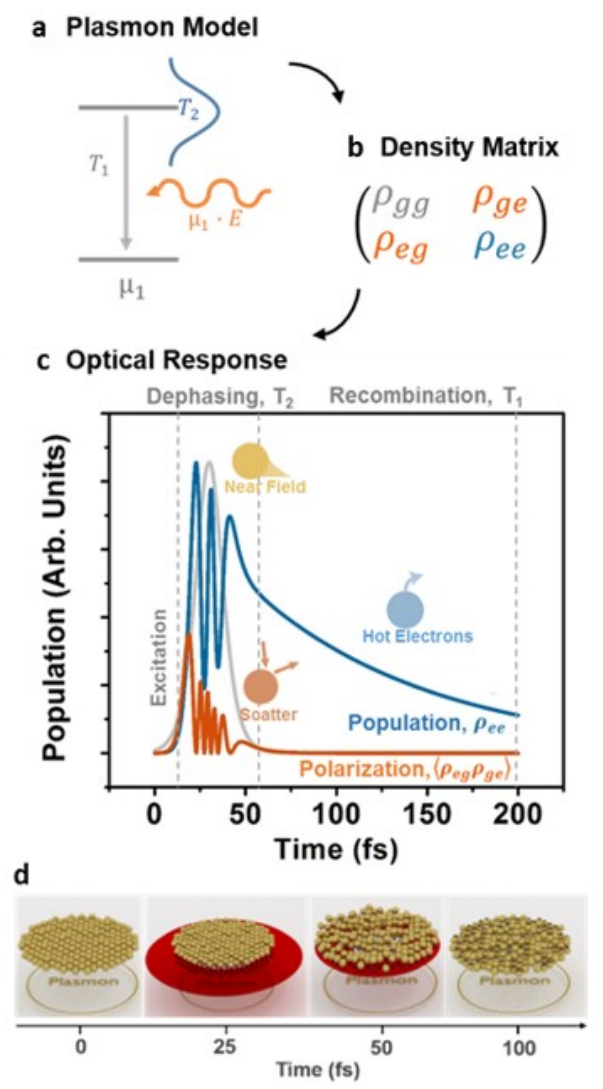

Figure 5.1 Schematic of density matrix model for describing the optical response of a plasmon. a, Dipole model of the plasmon including the dephasing, $T_{2}$, which leads to natural line broadening and the recombination, $T_{1} . \mathbf{b}$, The parameters from the dipole model are input into the density matrix, which describes the ground $\left(\rho_{g g}\right)$ and excited state $\left(\rho_{e e}\right)$ as the diagonal elements, and the coherences between the ground and excited state $\left(\rho_{e g}, \rho_{g e}\right)$ off-diagonal. c, The density matrix is solved using the quantum master equation, giving the time dependences of the plasmon's population and polarization after excitation. d, Schematic evolution of plasmon's progression in part $\mathbf{c}$.

The plasmon dephases less than 20-30 fs after excitation as the electron oscillations lose their collective nature ${ }^{9}$. During dephasing, the collective dipole moment decreases along with the near field interaction and scattering probabilities, with the incident energy converted into a hot carrier distribution. In Figure 5.1, the dephasing process is represented as the plasmon losing its coherence with the incident field, indicating energy is no longer shared between the plasmon's collective electron population and the photon field. This is reflected as a damping of the initial oscillations between polarization and population, and represents the decay of the near-field or scattering probability. After this stage, energy is no longer shared with a photon, only a hot electron population is left. As the plasmon dephases, the optical properties evolve from resonance behavior to that of independent electron-hole pairs, as if the metal had absorbed light away from resonance.

The plasmon's optical properties are therefore directly linked to how quickly and by what route the plasmon dephases. As the metal nanoparticle's size, shape, and constituent metal is switched, different nonradiative (surface, electron-electron, interband damping) ${ }^{13}$ and radiative (scatter) damping mechanisms become dominant. Just as the optical properties can be tuned spectrally by changing the size, shape, and metal of the nanoparticle, so can the balance between the near field, scattering, and hot electron or heat production and the corresponding solar energy conversion enhancement. 

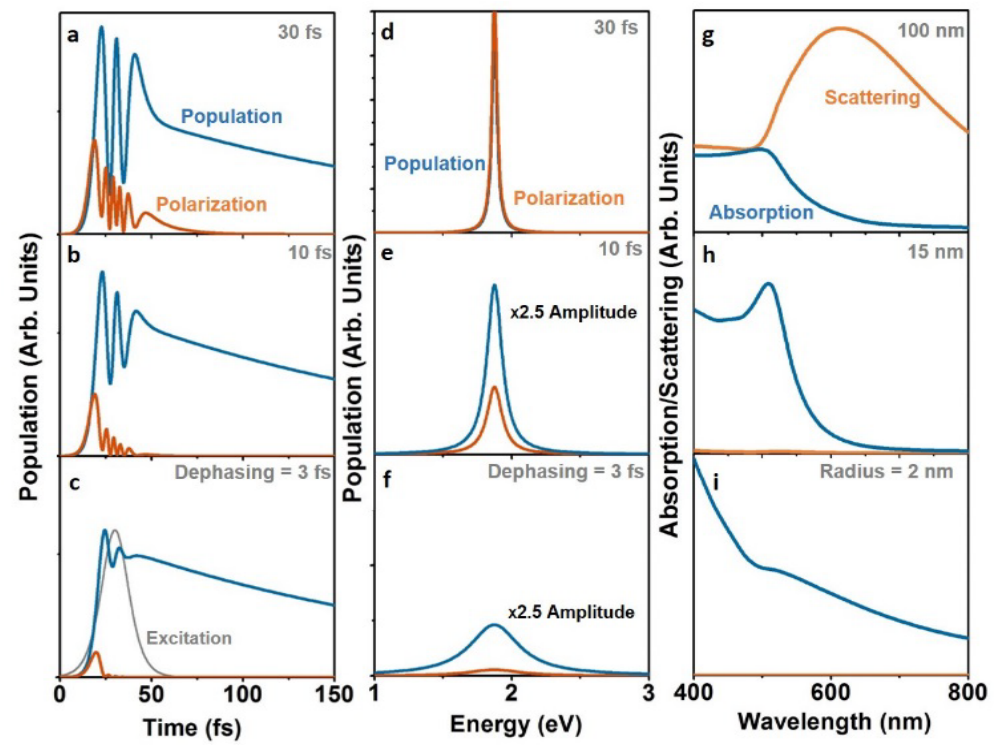

Figure 5.2. Effect of the plasmon's dephasing on optical response. a, The time evolution of the plasmon and the balance between the population and polarization (related to scatter) changes with increasing dephasing rate. b, For long dephasing times, scattering dominates, for short excitation times, the absorption dominates. The peak cross section also decreases and linewidth increases with increasing dephasing rate. c, The change in optical response with dephasing can be related to the change in the plasmon's absorption and scattering strengths with size.

Impact of Dephasing on Plasmon The link between commonly measured UV-Visible optical properties and the underlying dephasing times is demonstrated in Figure 5.2 by correlating the quantum-mechanical density-matrix model and a classical Mie theory model with a damping-modified dielectric constant. The balance between radiative and non-radiative damping processes is seen to determine the probability the plasmon will decay through near-field interactions, in which case no energy is left in the plasmon; scattering, in which case the energy is re-radiated to the far field; or hot carriers, in which case the energy is absorbed by the plasmon.

For example, radiative damping increases with volume and non-radiative interband damping decreases with decreasing resonance energy, so large nanorods and nanospheres (top panel Figure 5.2) have dephasings close to that of bulk metals. ${ }^{13}$ This means that the plasmon's electron oscillations stay collective longer, Figure 5.2a, storing energy in the near field until depolarization leads to scattering, Figure 5.2d, meaning scattering dominates over absorption (Figure 5.2g).

As the size of the nanoparticle is decreased to $\sim 15 \mathrm{~nm}$, the radiative efficiency decreases and nonradiative damping increases (Figure 5.2 middle panel). The plasmon only stays coherent with the incident field for several femtoseconds (Figure 5.2b), the scattering amplitude is decreased (Figure 5.2e), and a larger percentage of the plasmon's energy is likely to decay through the near field or be absorbed (Figure 5.2h). Accordingly, this size nanoparticle is heavily used in SERS for the local EM field enhancement. Further decreasing the nanoparticle's size (bottom panel Figure 5.2) to $3 \mathrm{~nm}$ continues this trend, with non-radiative damping dephasing the plasmon almost immediately into a hot electron distribution (Figure 5.2c) and creating a minimal plasmonic response (Figure 5.2f). In fact, the plasmon's absorption becomes close to the metal's (Figure 5.2i), with little near-field or resonance nature.

An important additional aspect is the impact of changing the plasmon's dephasing on the linewidth and oscillator strength. For solar energy, the plasmon must have a strong dipole moment for efficient scattering and absorption, but must also cover a broad portion of the solar spectrum. Contrary to this goal, the oscillator strength and linewidth have an inverse relationship with dephasing (Figure 5.2), so a balance 
between peak enhancement and spectral coverage must be found. This can be an important but overlooked effect when trying to optimize a plasmonic nanoparticle for solar energy conversion.

Although the density matrix calculations were performed in the dipole approximation, in which scattering cross sections of large nanoparticles are not always adequately described, the optical response was consistent with damping-modified dielectric constant Mie theory calculations (Figure 5.2) and the data of Reference 13 for nanorods and nanospheres.

Modeling in Dephasing Metal-Semiconductor System The interaction pathways between metal and semiconductor therefore do not exist independently. Hot electron transfer, scattering, and dipole-dipole coupling are all possible before the plasmon has dephased and must be treated coherently. After the plasmon dephases, hot electron transfer remains possible, re-emission of light weakly occurs by fluorescence through intraband transitions, and dipole-dipole coupling exists in the incoherent limit.

The density matrix model was thus extended to include interaction with a semiconductor by representing the semiconductor as a sum over interband dipole transitions weighted by the joint density of states (JDOS). ${ }^{38}$ The JDOS was representative of a direct band gap semiconductor (see Supplementary Calculations for further details), but with the finite linewidth of the interband dipole transitions leading to an Urbach tail, mimicking realistic semiconductors. ${ }^{39}$ The JDOS was scaled to give a semiconductor absorption of $50 \%$ of the incident light to represent a thin semiconductor film or nanoparticle. The resulting absorption (Figure 5.3) was representative of the $\mathrm{TiO}_{2},{ }^{26} \mathrm{Cu}_{2} \mathrm{O},{ }^{25}$ and $\mathrm{Fe}_{2} \mathrm{O}_{3}{ }^{27}$ commonly used in plasmonic samples.
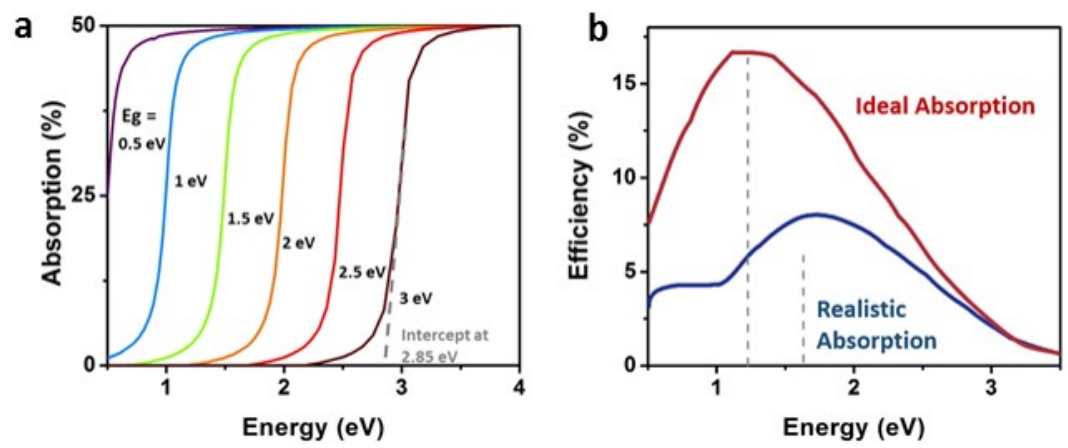

Figure 5.3. Absorption calculated using the density matrix model of the semiconductor for several band gaps. a, The absorption is calculated as the percent of incident light which created an excited state population in the steady-state limit. Note that the band gap is more representative of the initial rise of the absorption than the intersection with the axis due to the Urbach tail modeled, shifting the band gap $\sim 0.15 \mathrm{eV}$ from the intercept with the axis. $\mathbf{b}$, Since an absorption profile is used, and not an assumed $100 \%$ absorption at the band gap, the maximum efficiency calculations for the semiconductor alone are offset in energy. The ideal absorption is calculated using a Heaviside function at the band gap value scaled to $50 \%$ absorption. The realistic absorption is that in $\mathbf{a}$.

In other words, the semiconductor's photoconversion yield with or without the plasmon was found by solving the density matrix in the steady state limit for a spectrum of dipole's covering the incident excitation frequency range, then weighting each excited state population by the JDOS. Near-field interaction between the plasmon and semiconductor interband transitions were treated in a coupled-dipole approximation, scattering yields were used to predict re-absorption probabilities, and hot electron transfer was assumed to occur at a fixed rate from the remaining plasmon population. This approach allowed coherent effects, such as dephasing on resonance energy transfer and scattering versus absorption, as well as incoherent transfer schemes to be included. All possible plasmon-semiconductor enhancement pathways were therefore linked to a single parameter, the dephasing, allowing for optimization to be feasible. 
More specifically, the calculations were as follows for a given dephasing time, summarized in Figure 5.4. First, the incident power and frequency were taken from an AM1.5G spectrum, see Supplementary Information for spectrum reference. The response of the plasmon and semiconductor with and without coupling were then calculated by solving the density matrix using the quantum master equation. ${ }^{36}$ The semiconductor's excited state population versus frequency with dipole-dipole coupling gave the effect of PIRET. A dipole-dipole coupling of $0.001 \mathrm{fs}^{-1}$ was assumed, equivalent to a metal surface to semiconductor separation distance of $\sim 5-10 \mathrm{~nm}$ for a $\sim 10-15 \mathrm{~nm}$ metal nanoparticle. The plasmon's polarization gave the effect of scattering under the assumption that the plasmon re-reflected light not absorbed by the semiconductor. The plasmon's scattering peak at a dephasing of 8-10 fs was scaled so that $100 \%$ of rereflected light was trapped by the semiconductor, equivalent to the plasmon acting as a back-reflector with a dephasing dependent efficiency and linewidth. The plasmon's remaining excited state population after PIRET and scattering was multiplied by a constant transfer rate to represent hot electron transfer. The transfer rate was taken as $10 \%$ from the limiting value in Reference 40 , and the transferred carriers were added to the semiconductor's excited state population where the plasmon absorbed.

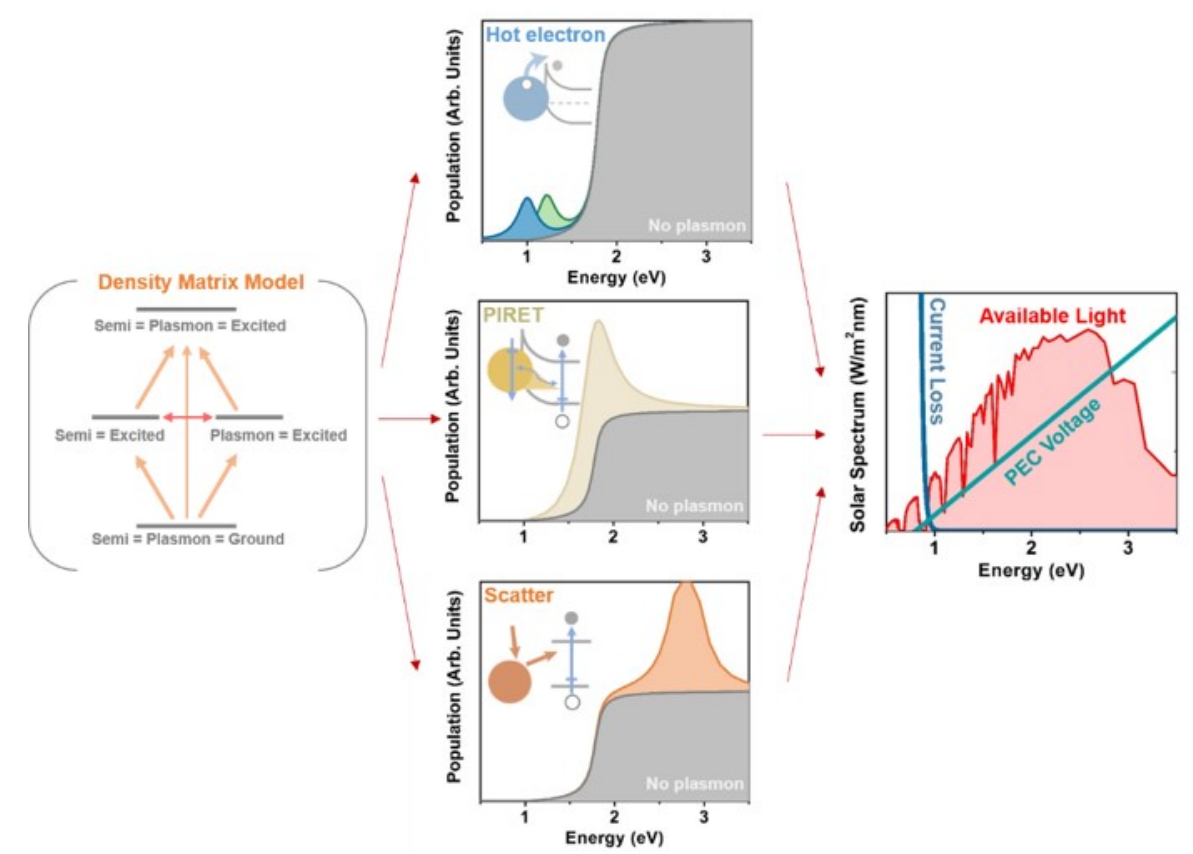

Figure 5.4 Schematic of maximum efficiency calculations using the density matrix.

The calculated plasmonic enhancement was next converted into a combined absorption profile for plasmon and semiconductor. The combined absorption profile was integrated over the solar spectrum to determine the number of photons absorbed, giving the photocurrent after thermalization losses were subtracted. The output power, determined by multiplying the photocurrent by the input voltage for PV and the band gap energy for PEC, then gave the overall efficiency after dividing by the AM1.5G solar spectrum. The thermal distributions of plasmon-excited carriers were assumed identical to the combined absorption for PIRET and scattering. For hot electron transfer, excited carriers were treated as if deposited over a Schottky barrier at energy of $E_{g} / 2+E_{\text {plasmon }}$ under the assumption of an intrinsic semiconductor.

The approximations made in this paper should be further detailed before reviewing the results. First, the effect of hot electron transfer on the population and subsequent effect on PIRET and scattering was 
neglected. This approximation was made because the $\sim 10 \%$ reduction in excited state population did not include the effect of increased interfacial dephasing through electron transfer, which can lead to decreases of $50 \%$ in efficiency, ${ }^{25}$ and in realistic situations hot electron transfer is expected to be closer to $1 \% .{ }^{40}$ Otherwise, the plasmon's population included the competing effects of scattering versus absorption versus dipole-dipole coupling. The change in refractive index of the semiconductor on optical response was also neglected, as well as the necessary sizes for the dipole-dipole treatment and near field effects to be fully valid. Finally, the heating of the plasmon and its effect on PV and PEC was neglected, but could be included in the future by assuming the remaining population of the plasmon is converted to phonons and heat.
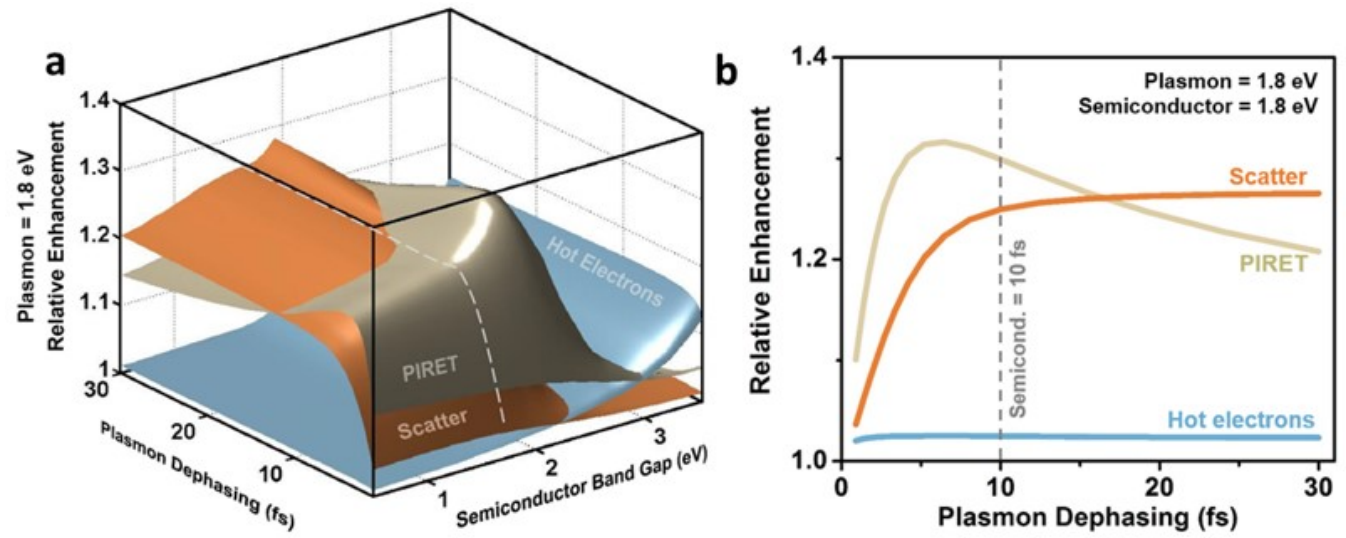

Figure 5.5. Relative enhancement semiconductor photoconversion by the plasmon. a, The relative enhancement versus the plasmon's dephasing and semiconductor band gap, defined by dividing the coupled-plasmonsemiconductor absorption integrated over the solar spectrum by the semiconductor absorption integrated over the solar spectrum. The plasmon energy was fixed at $1.8 \mathrm{eV}$. b, A cut-out of the 3D plot of part a at a semiconductor band gap of $1.8 \mathrm{eV}$, the semiconductor dephasing time is shown for comparison. The dashed line in a shows the approximate position of the cut in part $\mathbf{b}$.

\subsubsection{Results}

Photoconversion Enhancement The plasmonic enhancement pathways were first considered independent of each other, shown in Figure 5.5 for a plasmon energy of $1.8 \mathrm{eV}$ and a range of plasmon dephasings and semiconductor band gaps. The representative $1.8 \mathrm{eV}$ plasmon energy was chosen because it is near the energetic limit for water splitting and the peak intensity of the solar spectrum. The conclusions were the same for other plasmon energies, as reflected later in Figure 5.6 and 5.7. The interplay between dephasing and dominant plasmonic enhancement mechanism can be seen, as well as the ability of the plasmon to increase photoconversion above and below the semiconductor band edge. Figure 5.5 is scaled as the relative enhancement to photoconversion by the plasmon, determined by dividing the plasmoncoupled-semiconductor population by the semiconductor alone, such that an enhancement of 1.4 translates to the plasmon creating 1.4 times more carriers across for a spectrally flat spectrum than the semiconductor by itself.

Figure 5.5a shows scattering was most efficient at increasing photoconversion when the plasmon's dephasing was close to the value of bulk metals (20-30 fs) and the plasmon energy was greater than the semiconductor band edge. These dephasing times increased the scattering yield relative to absorption, as also seen in Figure 5.2, allowing more light to be trapped by reflection instead of being converted to heat in the metal by absorption. Again, this dephasing range corresponds to the metal nanoparticle being larger $\left(R \sim 50 \mathrm{~nm}\right.$ ) since radiative efficiency increases with volume. ${ }^{14-18}$ The plasmon energy was optimized above the semiconductor band edge because scattering only acts to increase absorption where the semiconductor 
can already absorb light. The largest scattering enhancement was therefore seen for a semiconductor with band gap at the $1.8 \mathrm{eV}$ plasmon resonance, matching the most intense portion of the solar spectrum. While these conclusions are for a single particle picture where increased dephasing narrows the plasmon's linewidth, the design conclusions of minimizing plasmon absorption and maximizing reflection are still congruent with those of more complex light-trapping structures.

Opposite to scattering, hot electron transfer created the largest increase in photoconversion when the semiconductor had an ultraviolet (UV) band gap and the plasmon absorbed light below band gap. Although smaller dephasing times mean a larger excited state population is created, with less energy lost to scattering and dipole-dipole coupling, the peak absorption intensity also dropped with increasing dephasing rates, see Figure 5.2, balancing any gains. The hot electron transfer enhancement was therefore relatively constant across the dephasing range tested in Figure 5.5, only decreasing slightly at both extremes. The optimal dephasing time for hot electron transfer was found to be 3-10 fs, consistent with the 10-20 nm nanoparticles most common experimentally. ${ }^{19-23}$ This conclusion does not take into account hot electron generation in the metal away from the plasmon resonance, or the dependence of hot electron and hole energy on metal nanoparticle size and interband transition energy, ${ }^{22}$ but the importance of balancing plasmon dephasing against overall absorption intensity is still a central design criteria for efficient hot electron enhancement.

Figure 5.5 also shows that PIRET increased photoconversion near/below the semiconductor band edge, only limited by the overlap of the semiconductor's and plasmon's absorption profiles. This trend is consistent with incoherent ${ }^{24}$ and coherent ${ }^{25}$ dipole-dipole coupling theory, which show resonance energy transfer is most efficient when the two dipole's linewidths overlap. The largest PIRET enhancement happened when the plasmon energy was slightly sub-band gap in the semiconductor's absorption tail. At this spectral position the plasmon's larger dipole moment allowed strong light absorption and subsequent population transfer to occur, extending the semiconductor's photoconversion.

Figure 5.5b reveals that PIRET was most efficient when the plasmon had a dephasing smaller but similar in magnitude to the semiconductor. Above and below this range, the efficiency dropped off quickly because of a balance of three effects. First, similar to hot electrons, the absorption intensity must be optimized against the linewidth to allow the maximum increase in solar energy conversion. Second, as the dephasing increased, more of the plasmon's energy was re-radiated as scatter, decreasing the available energy for dipole-dipole coupling. Third, during initial excitation the semiconductor and plasmon were in a coherence and shared the captured energy. This means that if the semiconductor and plasmon had identical dephasing times and dipole moments, the final incoherent populations would be equal. As the plasmon's dephasing time was decreased (increased) relative to the semiconductor, however, an energy transfer direction is defined since more (less) energy was trapped on the plasmon on average. The dependence of energy transfer direction on dephasing was experimentally seen in Reference 25.

Overall, PIRET led to the largest enhancement of the three mechanisms, exciting 1.3-1.4 times more carriers than the semiconductor alone, consistent with experimental enhancements. ${ }^{24-26,41,42}$ PIRET created the largest enhancement to the semiconductor's population because dipole-dipole coupling occurred at dephasing rates which correlated with broad plasmon linewidths. At these dephasings, the plasmon could absorb light over a wide spectral range while still transferring population to the semiconductor effectively, converting more of the solar spectrum. This is in contrast to optimized scattering, which in the density matrix model could only be achieved with limited linewidths, decreasing the number of photons captured. Hot electron transfer was maximal at broad linewidths like PIRET, but the weaker transfer rate limited the possible enhancement. 
PV and PEC Enhancement The relative enhancements of Figure 5.5 were next converted into a maximum solar energy conversion efficiency (Figure 5.6a for PV and Figure 5.6b for PEC), using the solar spectrum instead of the assumed flat spectrum. The efficiency of the plasmon-enhanced semiconductor is labelled on the contour lines. The efficiency of a semiconductor alone at the given band gap energy (constant in plasmon energy) is also labeled in parenthesis for comparison. This value is shifted from thickfilm SQ limits because of the semiconductor's absorption profile (Figure 5.3b). The dephasings which led to the maximum efficiency at several points are also marked, including a representative nanorod or nanosphere size from Reference 13. Further, the enhancement mechanism responsible for the given efficiency is shown as the color contour plot. All color contour plots are smoothed over the finite grid of semiconductor and plasmon energies used, so boundaries between mechanisms should not be taken as sharp.
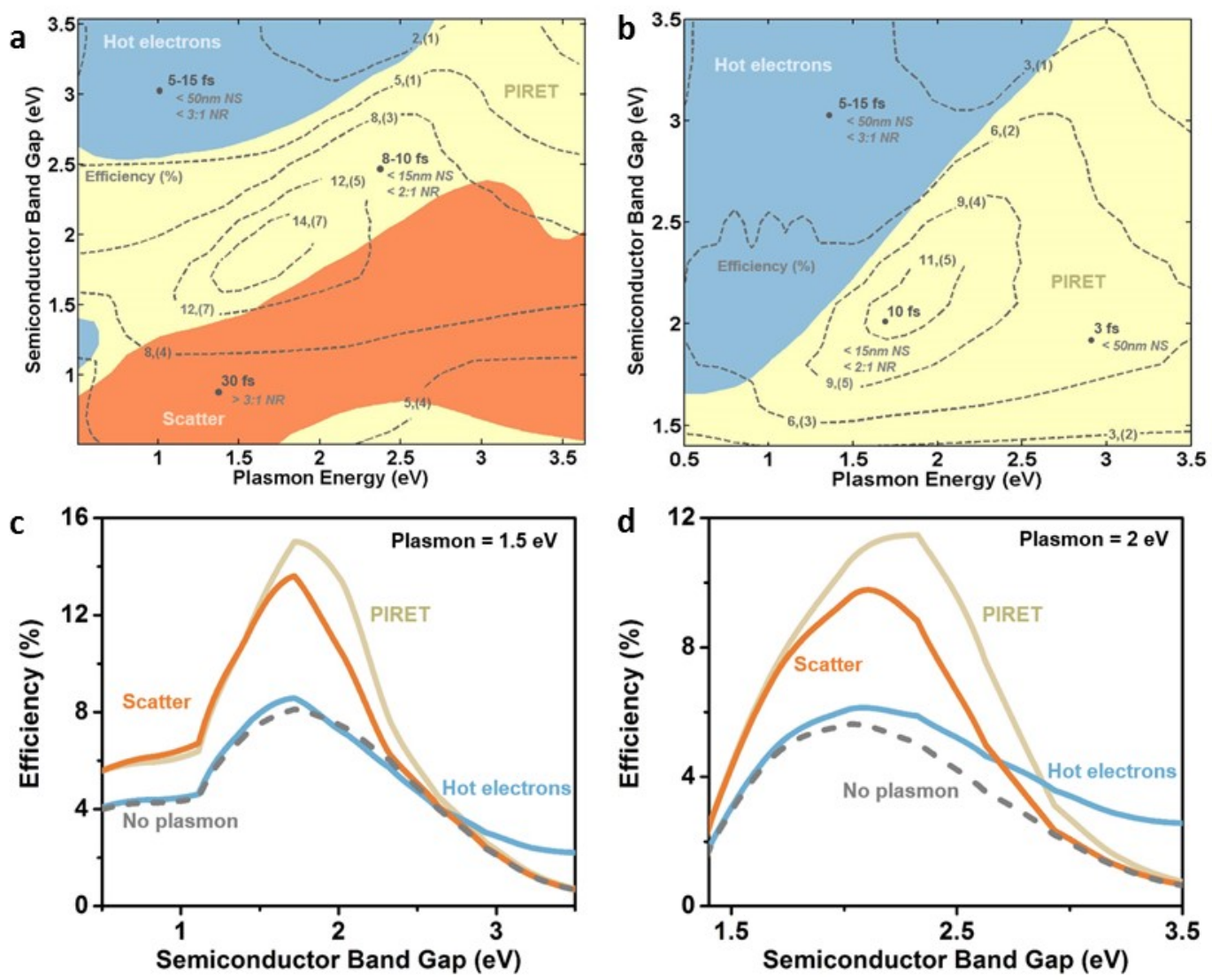

Figure 5.6. Maximum efficiency for solar energy conversion of an AM1.5G spectrum through a photovoltaics and $\mathbf{b}$ photo-to-chemical conversion. The semiconductor scale in $\mathbf{b}$ only goes to $1.4 \mathrm{eV}$ because the assumed $0.8 \mathrm{eV}$ in losses and $0.5 \mathrm{eV}$ offset from the absorption tail. The plasmonic enhancement mechanism responsible for the maximum conversion efficiency at a plasmon-semiconductor energy combination is shown. The maximum efficiency is labeled on each contour, along with the value from the semiconductor alone at that point in parenthesis. The dephasing at several points across the graph which led to the maximum enhancement is also shown. Ranges corresponding to the given dephasing times for Au nanosphere (NS) radius and Au nanorod (NR) aspect ratio are overlaid from Reference 13. A cut-out at $1.5 \mathrm{eV}$ and $2 \mathrm{eV}$ for the a photovoltaic and $\mathbf{b}$ photo-to-chemical conversion calculation is included.

The design conclusions of Figure 5.5 were reflected in the maximum-efficiency calculations of Figure 5.6, with enhancement factors shifted by the shape of the solar spectrum. For both PV and PEC, hot electron transfer was dominate for wide band gap semiconductors coupled to sub-band gap plasmons, with the plasmon's broad linewidth extending spectral coverage. Hot electrons doubled the efficiency of wide band 
gap semiconductors by a factor of 2 to 3 for the assumed $10 \%$ transfer rate. The largest hot electron transfer enhancement occurred for dephasing times which balanced absorption intensity versus linewidth. For PEC, the power was calculated from the short circuit current and the band edge voltage minus $0.8 \mathrm{eV}$ in losses ${ }^{3}$, including an additional $0.5 \mathrm{eV}$ offset to account for the band-edge tail. This translated to a larger hot electron enhancement for PEC than PV because smaller thermalization losses were present at short circuit. In PV a narrow range where a small band gap semiconductor was best enhanced by hot electrons also was found. This occurred because hot electron transfer deposited carriers in the semiconductor at a different energy than direct light absorption, avoiding some thermalization losses.

The balance of light absorption versus thermalization also modified the optimal conditions for PIRET and scattering compared to Figure 5.5. For PV, scattering was found dominant at plasmon energies above the semiconductor band edge, with dephasing times approaching the bulk metal. These dephasing times led to large scattering cross sections which increased semiconductor absorption where thermalization losses were smallest. Near the band edge, PIRET more efficiently captured and transferred light in the semiconductor's weak absorption tail than scattering. For PEC, PIRET dominated both above and near the band edge because the effects of thermalization were diminished at short circuit, and the broad plasmon linewidth better increased spectral coverage than scattering.
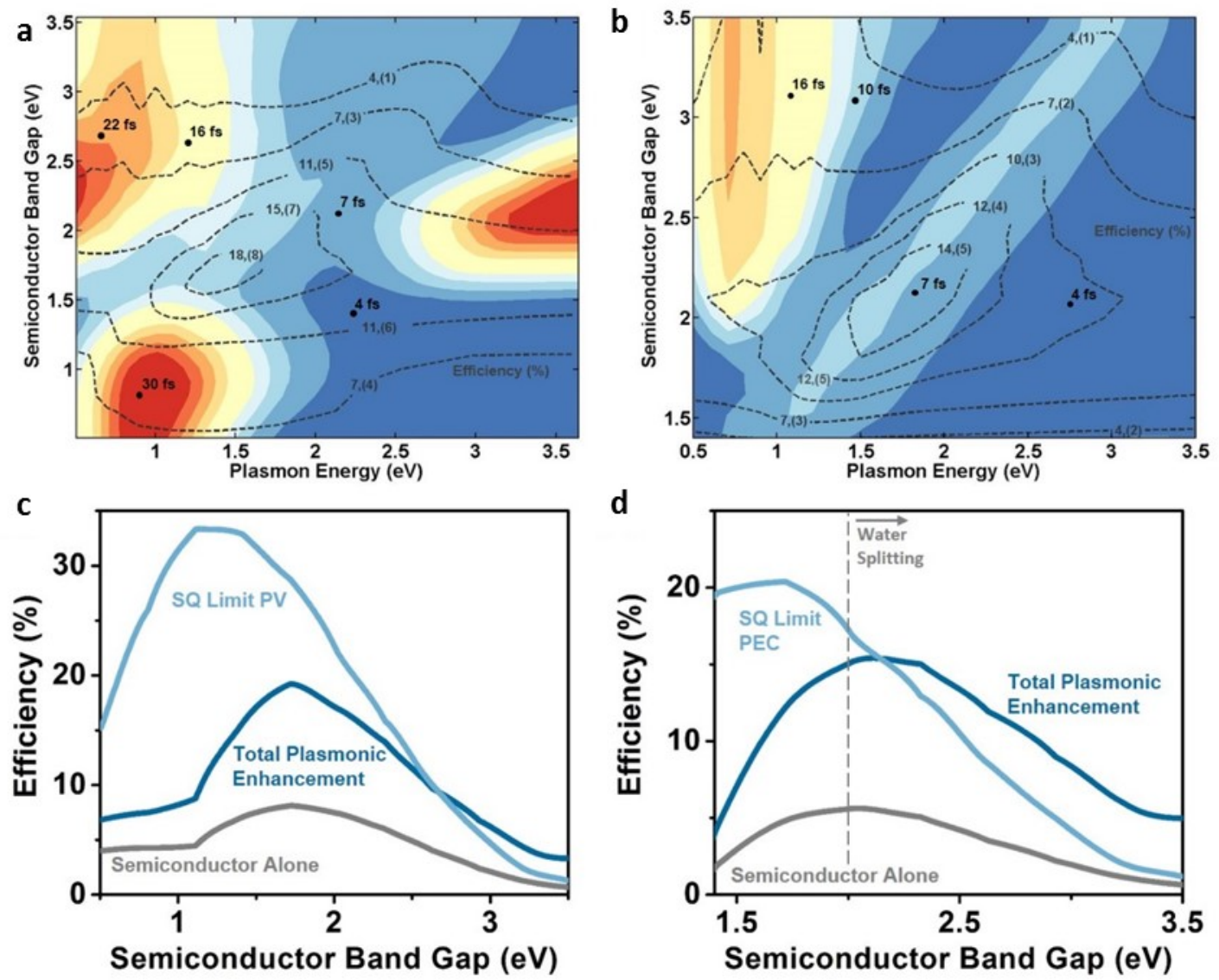

Figure 5.7. Maximum possible enhancement for solar energy conversion for a photovoltaics and $\mathbf{b}$ photo-to-chemical conversion. Whereas in Fig. 5.6 the plasmonic enhancement mechanisms are treated separately, in this figure the enhancement mechanisms act synergistically. The maximum efficiency is labeled on each contour, along with the value from the semiconductor alone at that point in parenthesis. Each color contour corresponds to a dephasing range, as labelled at several points across the graph. In $\mathbf{c}$ and $\mathbf{d}$ the maximum enhancement from an optimal plasmon energy and dephasing is compared to the semiconductor absorbing light by itself in the realistic model of the paper and assuming $100 \%$ absorption at the band edge. The $\sim 2 \mathrm{eV}$ band gap required for water splitting is indicated. 
Overall, PIRET led to the largest solar energy conversion efficiency compared to hot electrons and scattering. The peak PV and PEC efficiency was almost doubled when the plasmon was overlapped with the band edge and the plasmon's dephasing time was close to that of the semiconductor. Reference 13 shows that this condition can be met using Au nanorods tuned across the resonance range tested here and Au nanospheres with resonances tuned to less than $2 \mathrm{eV}(R<50 \mathrm{~nm})$.

It is important to note that the scattering amplitude was scaled to allow $100 \%$ light trapping at 8-10 fs, making scattering in PV slightly more efficient above the semiconductor band edge than PIRET. If the condition was relaxed to $100 \%$ light trapping at $15 \mathrm{fs}$, scattering became less dominant, showing the relative equivalence of PIRET and scattering for enhancements above, but not near, the semiconductor band edge. The equivalence occurs because both mechanisms act to increase the probability with which the semiconductor absorbs a photon, just through different radiative or non-radiative interactions.

In Figure 5.6 the three plasmonic enhancement mechanisms were optimized separately to show the best individual application. However, when a metal nanoparticle is combined with a semiconductor, all three eancement routes can co-exist and enhance photoconversion simultaneously. Figure 5.7 therefore shows the maximum efficiency for PV and PEC obtained considering scattering, hot electrons, and PIRET together. The dephasing that led to the peak enhancement is shown by the colored contours. The nanosphere and nanorod size range which can satisfy these dephasings from Reference 13 is the same as shown in Figure 5.6. When acting synergistically, the plasmon increased the peak maximum efficiency over two times to $18 \%$ for PV and 15\% for PEC from $8 \%$ and 5\% for the semiconductor alone. For PV and PEC, the maximum enhancement occurred for dephasing times of around $10 \mathrm{fs}$, indicating PIRET was still leading to the largest enhancement but with scattered light further increasing photoconversion. Inclusion of thermalization losses in PV again altered the optimal dephasing times and linewidths from PEC.

The ability of the plasmon to restore a weakly absorbing semiconductor to the SQ limit is seen in Figure 5.7c and 5.7d. For photovoltaics the thick-film limit was approached at band gaps larger than $\sim 1.5 \mathrm{eV}$. For photoelectrochemical cells, the thick-film limit was actually surpassed at band gaps larger than $1.8 \mathrm{eV}$, the energy necessary for water splitting. Plasmon-coupled-semiconductors can outperform the thick-film limit because the plasmon increases the light absorption spectral range, acting similar to a two semiconductor heterostructure. The thick-film limit is not surpassed in PV because thermalization losses balance the increase in spectral absorption.

\subsubsection{Discussion}

Figure 5.6 and 5.7 show the unique approach plasmonics gives to solar energy harvesting. A plasmon alone cannot efficiently drive the solar energy conversion process due to the short lifetime of excited intraband transitions. Instead, it very effectively acts as an antenna, absorbing a large amount of light in a small volume, and then using this energy to create carriers in the semiconductor both above and below the band gap. SQ limit calculations usually serve as an upper limit, as recombination losses require thin films

which cannot absorb all incident light. With the inclusion of plasmonics, thick-film like absorption can be reached while still using thin films and nanostructures that balance recombination losses. 


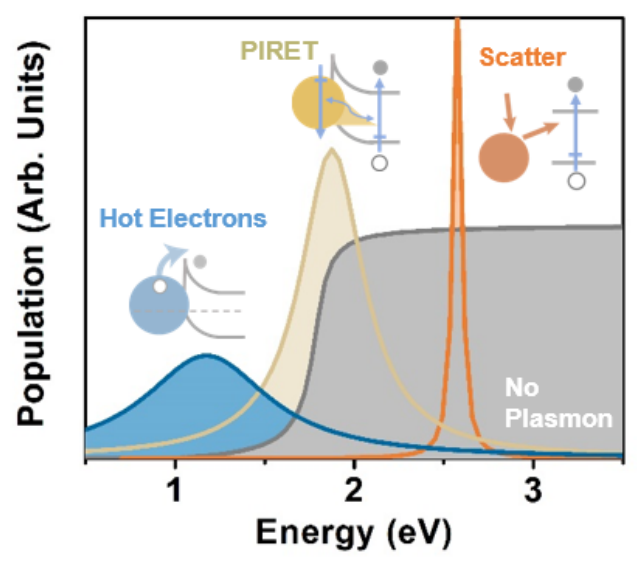

Figure 5.8. Representative schematic for where each plasmonic mechanism will lead to the largest enhancement in semiconductor photoconversion. The linewidths have been exaggerated in the schematic relative to the semiconductor absorption.

This goal, however, can only be achieved by selecting the proper enhancement mechanism and plasmon dephasing based on semiconductor band gap. The design guidelines gained from Figures this section are summarized in Figure 5.8, wherein the optimal use of each plasmonic enhancement mechanism is represented. Again, it must be remembered these conclusions are for a single particle, or a per-volume, based enhancement. In more complex architectures light trapping can lead to 100\% trapping across the solar spectrum, ${ }^{14-18}$ and although the transfer efficiency of hot electrons remains around $1 \%$ in most plasmonic nanoparticles, ${ }^{19-23}$ possibilities to reach $30 \%$ have been shown ${ }^{43}$. However, the same argument can be made for applying more complex structures to dipole-dipole coupling, increasing its transfer rate as well. Therefore, the important conclusion of Figure 3-5 is that when considered on a per-volume basis, the plasmon's near field will lead to the largest enhancement in photoconversion.

How to optimize plasmonic nanoparticles for a particular dephasing should also be commented on. The dephasing range used in this paper covers 0.1-30 fs, which at the high side is on the scale of bulk Au or Ag, indicating a plasmonic nanoparticle with little additional damping, and on the low side covers heavy damping on length scales where quantum effects could occur. Within this range, the dephasing is most easily selected by tuning nanoparticle size, shape, and metal. Nanoparticle size determines dephasing by the balance between surface damping effects in small nanoparticles versus the increase in radiative efficiency with metal volume. The size also determines the resonance position relative to the interband transition threshold of a given metal. For a similar resonance and size, Reference 13 showed the nanoparticle shape is equally important, with nanospheres having a quicker dephasing time than nanorods.

These guidelines can be used to qualitatively select a nanoparticle shape, however more complex geometries require direct measurement of dephasing times. This is difficult, as single-particle measurements or similar complex experimental techniques are necessary to accurately determine dephasing times within the inhomogenously broadened experimental linewidth. As a substitute, finite difference time domain (FDTD) or other classical EM calculations can be used to estimate the dephasing time of complex structures through the linewidth $(\Gamma)$ by $T_{2}=2 \hbar / \Gamma$ where $2 \hbar=1316 \mathrm{fs} \cdot \mathrm{meV} .{ }^{13}$ Simulation can be used to predict an optimal structure to match the dephasing times given in this paper, then verified by comparison to experimental absorption and characterization. This procedure is already common in predicting spectral range and local electromagnetic field enhancements for semiconductor photoconversion, but should be extended to selecting the appropriate dephasing and plasmonic enhancement mechanism. 
The predictions were also compared against representative results for experimental systems to verify the model. It is difficult to compare the absolute performance because it depends on the complete solar system's efficiency and not just the plasmon. Instead, the ratio of the total solar energy conversion efficiency with and without the plasmon was compared against Figure 5.6 and 5.7. An estimated dephasing of 1-3 femtoseconds was used to be consistent with most plasmonic geometries. For photovoltaic cells with band gaps of $\sim 1.8 \mathrm{eV}$ and plasmon energies of $\sim 1.8 \mathrm{eV}^{44}$ and $\sim 3 \mathrm{eV}^{45}$ in a scattering configuration, enhancements of $\sim 1.2$ were reported, consistent with the 1.2-1.4 times enhancement predicted from Figure 5.6 when the $60 \%$ reflection efficiencies from the experimental geometries is taken into account. For a PEC using hot electrons, usually relying on $\mathrm{TiO}_{2}$ with a band gap of $3.2 \mathrm{eV}$ and a $\mathrm{Au}$ plasmon with energy $\sim 2 \mathrm{eV}$, the enhancements have consistently been less than $1 \%^{21,23}$. If the upper-limit $10 \%$ transfer rate used in calculating Figure 5.6 is adjusted to the $1 \%$ or less reported, this changes the enhancement predicted from 3 times to $<1.1$ times, in line with experimental values. For PIRET with a plasmon energy $\sim 1.9 \mathrm{eV}$ and semiconductor band gap of $\sim 1.8 \mathrm{eV}^{25}$, an enhancement of 2.3 was reported by integrating the action spectrum photocatalysis over the solar spectrum. Although photocatalysis was not calculated, this is close to the unassisted PEC shown in Figure 5.6, which predicts an enhancement of 2.2 times, in line with experimental value.

These experimental results validate the model, but also point to a larger theme. Only PIRET is optimized in the 1-3 fs dephasing range of most plasmonic nanoparticles, and correspondingly the experimental enhancement is the largest, congruent with the theoretical predictions. For scattering and hot electrons, the optimal dephasing range is not reached. Therefore, if plasmonic enhancements are to be maximized, more attention must be paid to tuning the dephasing, in addition to choosing the appropriate plasmon energy and enhancement mechanism.

In summary, the possible plasmonic enhancement mechanisms were unified in a predictive model using a density matrix approach, allowing the relative scattering, dipole-dipole, and hot electron enhancements to be calculated including the effects of dephasing and coherence. This approach revealed the optimal dephasing for each enhancement mechanism, as well as what plasmon energy and mechanism will lead to the largest enhancement in photoconversion for a given semiconductor band gap. Scattering dominated at dephasings close to the bulk metal when the plasmon had an energy above the semiconductor's band gap. Hot electrons dominated for wide band gap semiconductors when the plasmon had a small energy and a dephasing that balanced absorption intensity and linewidth. PIRET led to the largest enhancement when the plasmon's dephasing was close to the semiconductor and the plasmon was overlapped with the band edge. In general, the largest enhancement is possible by using PIRET and scattering in conjunction to increase photoconversion near the band edge. The results presented will simplify design and optimization of plasmonics for solar energy enhancement in PV and PEC by allowing the best plasmon dephasing and energy to be selected for a given semiconductor band edge.

\subsubsection{Supplementary Calculations}

\section{Extending Density Matrix to Semiconductor}

In order to calculate the plasmonic enhancement the density matrix was solved using the quantum master equation as outlined in Reference 36. The treatment included the recombination time $T_{1}$ and the dephasing

time $\frac{1}{T_{2}}=\frac{1}{2 T_{1}}+\frac{1}{T_{2}^{*}}$. For the dephasing time, the factor of two times $T_{1}$ ensures the correct decay rate of the population and $T_{2}^{*}$ is pure dephasing, see Ref. 37. Following the formalism of Reference 36, the single particle correlations are solved in terms of spin operators in the time domain using a numerical procedure, 
or in the steady state by solving the resultant linear system of equations. The relevant quantities for calculation of the enhancement to solar energy conversion is then the excited state population $<\frac{1}{2}+S_{i}^{z}>$ of the plasmon and the semiconductor, as well as the polarization of the plasmon $\left\langle S_{i}^{+} S_{i}^{-}\right\rangle$which is proportional to the scattered field intensity.

The formalism of Reference 36 is for a single plasmon and semiconductor interband dipole. To replicate a semiconductor's absorption, a range of excited state populations at frequencies covering the full solar spectrum was first calculated, and then the excited state population at each frequency was multiplied by the joint density of states (JDOS) after the manner of Ref. 38. The JDOS was taken as

$$
\operatorname{JDOS}(\hbar \omega)=A * \Theta\left(\hbar \omega-E_{g}\right)
$$

where $\hbar \omega$ is energy, $\Theta(x)$ is the Heaviside function, $E_{g}$ is the band gap, and the scaling factor $A=30$ taken to make the plasmon and semiconductor absorption close at 3 fs plasmon dephasing, similar to the coreshell nanoparticles often used in plasmonics. The absorption tail of the Lorentzians which represent individual interband transitions in the semiconductor naturally creates an Urbach-like tail, modeling singlesemiconductor absorption profiles common in literature. However, a $0.2 \mathrm{fs}^{-1}$ cut-off is necessary for each individual interband transition to avoid excessive absorption beyond the semiconductor band edge. These parameters can be modified as needed depending on the desired semiconductor absorption profile.

\section{Calculating Enhancement from Plasmon}

The plasmonic enhancement was calculated from the density matrix results. First the populations and polarization of the semiconductor and plasmon were output for a frequency range covering the AM1.5G spectrum with the incident power given by the AM1.5G spectrum. At each frequency or energy to be tested the populations and polarizations were solved with and without dipole-dipole coupling in order to calculate the relative enhancements.

Resonant Energy Transfer- The excited state population created by resonant energy transfer was taken as the excited state population of the semiconductor with dipole-dipole coupling to the plasmon. This was converted into an effective absorption by dividing the excited state population versus frequency by the incident power, scaled by a factor that makes the semiconductor without coupling have a peak absorption of $50 \%$. In equation form this reads

$$
\alpha_{\text {PIRET }}(\hbar \omega)=B * \frac{<\frac{1}{2}+S_{\text {semi }+ \text { plasm }}^{Z}>}{N_{A M 1.5 G}}
$$

where $B=-\ln (0.5) / \max \left(<\frac{1}{2}+S_{\text {semi }}^{z}>/ N_{A M 1.5 G}\right)$ is the calibration factor which makes

$$
A b s_{\text {semi }}=1-\exp \left(-\alpha_{\text {semi }}\right)=50 \%
$$

at the maximum of the semiconductor's absorption. The effective absorption of the plasmon is then

$$
\operatorname{Abs}_{\text {PIRET }}(\hbar \omega)=1-\exp \left(-\alpha_{\text {PIRET }}(\hbar \omega)\right)
$$

with $S_{\text {semi+plasm }}^{Z}$ corresponding to the spin operator for the semiconductor coupled to the plasmon, $S_{\text {semi }}^{Z}$ corresponding to the spin operator for the semiconductor without coupling, and $N_{A M 1.5 G}$ the energy dependent photon density from the AM1.5G spectrum. The 50\% absorption at peak of the semiconductor corresponds to a specific absorption cross section times a thickness.

Hot electrons- The excited state population created by hot electrons was taken as the plasmon excited state population with dipole-dipole coupling times a transfer rate, plus the excited state population of the semiconductor without dipole-dipole coupling or scattering. Again, this was converted into an effective absorption by dividing the combined population versus frequency by the incident power, scaled by a factor that made the semiconductor without coupling have a peak absorption of $50 \%$. In equation form this reads 


$$
\alpha_{H E}(\hbar \omega)=B * \frac{<\frac{1}{2}+S_{\text {plasm }+ \text { semi }}^{Z}>* \Gamma_{H E}+<\frac{1}{2}+S_{\text {semi }}^{Z}>}{N_{A M 1.5 G}}
$$

giving

$$
A b s_{H E}(\hbar \omega)=1-\exp \left(-\alpha_{H E}(\hbar \omega)\right)
$$

with $S_{\text {plasm }+ \text { semi }}^{Z}$ corresponding to the spin operator for the plasmon coupled to the semiconductor, $\Gamma_{H E}$ corresponding to hot electron transfer rate taken as $10 \%$ from Ref. 40 , and $S_{\text {semi }}^{Z}$ corresponding to the spin operator for the semiconductor without coupling.

Scattering- The enhancement in semiconductor absorption by scattering was calculated by outputting the polarization of the plasmon when coupled by dipole-dipole interactions to the semiconductor, then scaling the amplitude of the polarization. The scaling factor, $C=1500 * B$, was chosen so that at 8-10 fs plasmon dephasing the semiconductor without dipole-dipole coupling would absorb almost all light scattered by the plasmon, representing multiple reflections or a light trapping efficiency of $100 \%$ at the plasmon's scattering peak. In equation form this reads

$$
\alpha_{\text {scatt }}(\hbar \omega)=C * \frac{<S_{\text {plasm }+ \text { semi }}^{+} S_{\text {plasm }+ \text { semi }}^{-}>}{N_{A M 1.5 G}}
$$

where $<S_{\text {plasm }+ \text { semi }}^{+} S_{\text {plasm }+ \text { semi }}^{-}>$is the polarization of the plasmon coupled to the semiconductor. Equation S7 therefore corresponds to some given number of reflections, of which can allow the semiconductor to re-absorb light not converted on the first pass, as given by

$$
A b s_{\text {Scatt }}(\hbar \omega)=\left[1-\exp \left(-\alpha_{\text {semi }}(\hbar \omega)\right)\right]+\left[\begin{array}{c}
\left(1-\left(1-\exp \left(-\alpha_{\text {semi }}(\hbar \omega)\right)\right)\right) * \ldots \\
\left(1-\exp \left(-\alpha_{\text {scatt }}(\hbar \omega) * \alpha_{\text {semi }}(\hbar \omega)\right)\right)
\end{array}\right]
$$

It should be noted $C=1000$ was used for $100 \%$ light trapping at 15 fs.

Through this method, an effective absorption was created that considered the enhancement of each mechanism independently on the semiconductor but through a single plasmon source. As noted in the previously, the losses from hot electrons were neglected because interface damping can have a much larger effect.

For the combined calculation in Figure 5.7 the same approach was taken, but now combining the hot electron population to the semiconductor absorption with dipole-dipole coupling, and taking this as the initial absorption in Equation 5.8. The scattering then causes multiple reflections

$$
\begin{gathered}
A b s_{\text {overall }}(\hbar \omega)=\left[1-\exp \left(-\left(\alpha_{\text {PIRET }}(\hbar \omega)+\alpha_{H E-\text { only }}(\hbar \omega)\right)\right)\right]+\cdots \\
{\left[\left(1-\left(1-\exp \left(-\left(\alpha_{\text {PIRET }}(\hbar \omega)+\alpha_{H E-\text { only }}(\hbar \omega)\right)\right)\right) *\left(1-\exp \left(-\alpha_{\text {scatt }}(\hbar \omega) * \alpha_{\text {semi }}(\hbar \omega)\right)\right)\right]\right.}
\end{gathered}
$$

where $\alpha_{H E-\text { only }}(\hbar \omega)=B * \frac{<\frac{1}{2}+S_{\text {plasm+semi }}^{Z}>* \Gamma_{H E}}{N_{A M 1.5 G}}$ to avoid double counting the semiconductor from Equation 5.5.

\section{Calculating Solar Energy Conversion}

Once the effective absorption of the plasmon was calculated, this was turned into a conversion efficiency by first determining the number of photons absorbed

$$
N_{\text {photons }}=\int d(\hbar \omega) A b s_{i}(\hbar \omega) * N_{A M 1.5 G}(\hbar \omega)
$$

where $A b s_{i}(\hbar \omega)$ is the effective absorption to be used from the above enhancement mechanisms and $N_{A M 1.5 G}(\hbar \omega)$ is again the photon density per energy in the AM1.5G spectrum. The loss due to carrier thermalization was then included as 


$$
R_{0}=\frac{2 \pi}{c^{2} h^{3}} * \int d(\hbar \omega) A b s_{i}(\hbar \omega) * \frac{(\hbar \omega)^{2}}{\exp \left(\frac{\hbar \omega}{K_{b} T}\right)-1}
$$

where $c$ is the speed of light, $h$ is Plank's constant, $K_{b}$ Boltzmann's constant, and $T$ the temperature of the solar cell. For heating losses the effective absorption was used, except in the case of hot electrons where the absorbed carriers were offset $E_{g} / 2$ from the plasmon frequency when added to the semiconductor to represent an intrinsic Schottky barrier.

For photovoltaics the current density and maximum efficiency were determined by

$$
J(V)=q *\left(N_{\text {photons }}-R_{0} * \exp \left(\frac{V}{K_{b} T}\right)\right)
$$

and

$$
E f f_{P V}=\max (V * J(V)) / P_{A M 1.5 G} \text { total }
$$

where $P_{A M 1.5 G_{\text {total }}}$ is the total power in the AM1.5G spectrum. For solar to chemical conversion this is modified to

$$
E f f_{P E C}=J(0) *\left(E_{g}-0.8-0.5\right) / P_{A M 1.5 G} \text { total }
$$

where the $0.8 \mathrm{eV}$ loss is intrinsic losses including thermodynamics effects as justified in Reference 3 , and the $0.5 \mathrm{eV}$ factor takes into account the absorption tail.

\section{Overall Calculation Flow and Input Parameters}

The overall calculation flow is represented in Figure 5.4 and was repeated for a range of plasmon energies, semiconductor band gaps, and plasmon dephasings. The resulting data set was then used to find the maximum conversion efficiencies as reported in the main text.

The input parameters were unless otherwise noted: $\mu_{\text {plasmon }}=1.5 e-27 \mathrm{Cm}$ estimated from Ref. 46 and $T_{1 \text { plasm }}=1 \mathrm{ps}$ estimated from average electron-phonon relaxation times in Ref. $47 ; \mu_{\text {semiconductor }}=$ $1 e-28 \mathrm{Cm}$ estimated from Ref. 48 and also as roughly charge times the lattice constant, with $T_{1_{\text {semi }}}=$ $1 p s$ estimated from Ref. 49 and $T_{2}^{*}=10 f s$ estimated from Ref. 50 and 51 . The input field strength varied as the AM1.5G spectrum. The close distance of the plasmon and semiconductor allowed the cooperative emission term to be neglected as justified in Ref. 38. The dipole-dipole coupling was taken as $V=0.001 \mathrm{fs}^{-}$ ${ }^{1}$, which given the dipole moments used, corresponds to an orientation averaged separation of $\sim 20 \mathrm{~nm}$ between the plasmon and semiconductor centers. Given that most plasmonic nanoparticles with 10-15 nm in radius have a few fs dephasing time, and that the size effects are being neglected in this approximation, this corresponds to a plasmon to semiconductor surface distance of $\sim 5-10 \mathrm{~nm}$.

\subsection{Engineering and Controlling Plasmonic Enhancement Mechanisms}

If the goals of Section 5.2 are to be met to create high efficiency plasmonic devices, it must first be known how to control the different plasmonic enhancement mechanisms. One route, as introduced in the previous section, is by using the plasmon's dephasing to adjust the dominant optical response. However, even once this is done, as shown in Chapter 2-4, it can still be best to physically isolate the chosen enhancement mechanism by structure design to prevent unwanted recombination or dephasing routes. Given that scattering can be treated as an independent metal and semiconductor, and thus is easily separated by large insulating barriers or even free space, in this section we focus on how to control hot carrier injection and PIRET which depend on the coupled metal-semiconductor system and are known to co-exist in the metal nanoparticles commonly used in literature. 
It is well known that hot electrons can overcome the metal-semiconductor Schottky barrier. Efficient hot electron injection requires direct contact between the plasmonic metal and the semiconductor (Chapter 3). In contrast, PIRET is possible as long as spectral overlap exists between the LSPR and the absorption band of the semiconductor, and the semiconductor is located spatially within the plasmon's near-field (Chapter 4). Spectral overlap and physical contact are therefore the two key factors governing the hot electron injection and PIRET processes.

In this Section ${ }^{26}$ a set of metal@ $\mathrm{TiO}_{2}$ core-shell nanoparticles with varying spectral overlap and contact between the metal and the semiconductor are thus designed to show that the different plasmonic energy transfer processes can be controlled. This is shown in four different types of metal@ $\mathrm{TiO}_{2} \mathrm{systems} \mathrm{as}$ follows: In $\mathrm{Au@} \mathrm{TiO}_{2}$ core-shell nanoparticles, charge carrier injection follows the plasmon's absorption, indicating hot electron transfer to $\mathrm{TiO}_{2}$ from $\mathrm{Au}$. In $\mathrm{Ag} @ \mathrm{SiO}_{2} @ \mathrm{TiO}_{2}$, hot electron injection is impossible, but the charge carrier creation follows the spectral overlap of PIRET. Both the hot electron injection and PIRET could happen in $\mathrm{Ag@} \mathrm{TiO}_{2}$, while neither hot electron injection nor PIRET can occur in $\mathrm{Au} @ \mathrm{SiO}_{2} @ \mathrm{TiO}_{2}$, preventing plasmon-enhancement of photoconversion in $\mathrm{TiO}_{2}$. Using these results, a chart is developed to map the possible plasmonic enhancement mechanisms in metal-semiconductor heterojunctions.

\subsubsection{Methods}

Au nanoparticles were synthesized based on a citrate-reducing methodology. ${ }^{23}$ Typically, $100 \mathrm{~mL}$ $0.01 \mathrm{wt} \% \mathrm{HAuCl}_{4}$ aqueous solution was heated to boil and then $0.7 \mathrm{~mL} 1 \mathrm{wt} \%$ sodium citrate aqueous solution was added. The mixture quickly changed to wine red color, and was kept boiling for 30mins to ensure the complete reduction of $\mathrm{HAuCl}_{4}$. Ag nanoparticles were next synthesized with a similar reduction method. ${ }^{41} 150 \mathrm{~mL}$ of $1 \mathrm{mM} \mathrm{AgNO}_{3}$ aqueous solution was heated to a boil, and $10 \mathrm{~mL}$ of $1 \mathrm{wt} \%$ sodium citrate solution was added. The mixture was the kept boiling for $30 \mathrm{~min}$.

The metal@ $\mathrm{TiO}_{2}$ core-shell nanoparticle was also made following a published procedure. ${ }^{52}$ Assynthesized metal nanoparticles were wrapped with a polymer layer by adding $0.5 w t \%$ hydroxypropyl cellulose and stirring for overnight. The modified metal nanoparticles were then dispersed in 4-time volumes of isopropanol. A proper amount of ammonia and $10 \mathrm{mM}$ titanium oxide precursor isopropanol solution were added. The reaction occured for 6 hours with the products collected by centrifuge and washed with isopropanol and ethanol. Metal@SiO 2 was synthesized by the Stöber method. ${ }^{53}$ Metal nanoparticles were first modified with Polyvinylpyrrolidone (PVP, 55 000), and then dispersed in an isopropanol solution. A proper amounts of ammonia and TEOS was then added, with the $\mathrm{SiO}_{2}$ shell thickness controlled by varying the TEOS concentration and reaction time. The synthesis of the sandwich structure then followed the same procedure as that for metal@ $\mathrm{TiO}_{2} .^{52}$ As-synthesized metal@SiO 2 particles were modified with HPC, and then reacted in the titanium oxide precursor solution. The products were collected with a centrifuge and washed with isopropanol and ethanol.

The light absorption spectra were obtained with a Shimadzu 2550 spectrometer in aqueous solution. The morphology for core-shell structure was confirmed with a JEOL 7600F field transmission electron microscope (TEM). The transient absorption spectroscopy was measured using pulses from a $1 \mathrm{kHz}$ Ti:Sapphire Amplifier. The samples were tested in solution and stirred. The range of pump wavelengths were made using sum frequency generation or frequency doubling of an optical parametric amplifier output. The pump power was kept at $\sim 8 \mathrm{~mJ} / \mathrm{cm}^{2}$ for all wavelengths, with each measurement normalized for small changes in power as discussed in the text. The transient absorption signal was measured in a stirred cuvette using a chopper and a lock-in amplifier. The maximum transient signal was determined by using a robust 
second order polynomial fit, with error bars shown as the functional predictive bounds at a 95\% confidence level.

\subsubsection{Results}

Figure 5.9a show a schematic of the samples. TEM images and characterization are in Reference 26. As seen in the extinction spectra in Figure 5.9b, a Ag core allowed spectral overlap between the plasmon and the absorption tail of $\mathrm{TiO}_{2}$. Spectral overlap existed both with and without the $\mathrm{SiO}_{2}$ insulating barrier, whose $10 \mathrm{~nm}$ thickness is sufficient to block hot electron transfer but still allowed coupling through the plasmon's near field. ${ }^{24}$ Similarly, a Au core had no spectral overlap with $\mathrm{TiO}_{2}$, with the spectral response below $500 \mathrm{~nm}$ dominated by interband transitions.
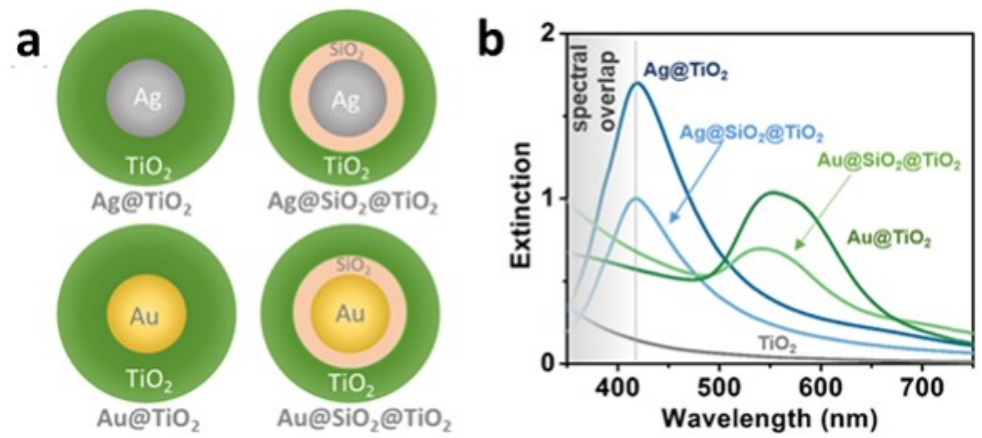

Figure 5.9. Core@shell metal nanoparticles for controlling plasmonic enhancement. a, Schematic of metal core@ $\mathrm{TiO}_{2}$ shell nanoparticles. b, Extinction of each metal core@ $\mathrm{TiO}_{2}$ shell nanoparticle as well as $\mathrm{TiO}_{2}$ alone. The cut-off region for significant spectral overlap is marked. The existence or lack of spectral overlap or insulating $\mathrm{SiO}_{2}$ barrier allows the different plasmonic enhancement mechanisms to be controlled.

Transient absorption spectroscopy was used to experimentally determine the plasmonic enhancement mechanism. In this technique, a strong pump pulse first excited the plasmon, and then the amount of charge carriers created by the plasmon in the semiconductor was determined by probing the $\mathrm{TiO}_{2}$ with an $800 \mathrm{~nm}$ pulse. The $800 \mathrm{~nm}$ probe pulse measured electron trap states in the $\mathrm{TiO}_{2}$. The trap states' filling after excitation has been shown to correlate well with PEC and photocatalysis performance. ${ }^{54}$ Transient absorption spectroscopy was chosen since it allows the transfer and creation of carriers to be measured on a picosecond time scale, before recombination can reduce the carrier population. In comparison to action spectrum or IPCE measurements, this guarantees that all carriers created in the semiconductor by the plasmon are measured, instead of only the carriers capable of driving a solar energy process.

It is difficult to remove the background contributions in transient absorption spectra obtained from the plasmonic metal-semiconductor heterojunctions. In this work, this problem was overcome by probing the complex equilibration that existed for nanoparticles in solution when additional charge was excited in the trap states of the $\mathrm{TiO}_{2}$. When $\mathrm{TiO}_{2}$ or Au alone was excited by the pump pulse, the transient signal was negative (Figure 5.10b). The negative change in transmission corresponded to more light being absorbed from the probe beam. For $400 \mathrm{~nm}$ excitation of $\mathrm{TiO}_{2}$, the increase in absorption occurred because more carriers were promoted into the trap states probed. For $510 \mathrm{~nm}$ excitation of $\mathrm{Au}$, thermalized carriers were created from electron-phonon scattering, leading to an increase in free carrier absorption. The $\mathrm{Au}$ and $\mathrm{TiO}_{2}$ curves were smoothed for comparison in Figure 5.10b. 

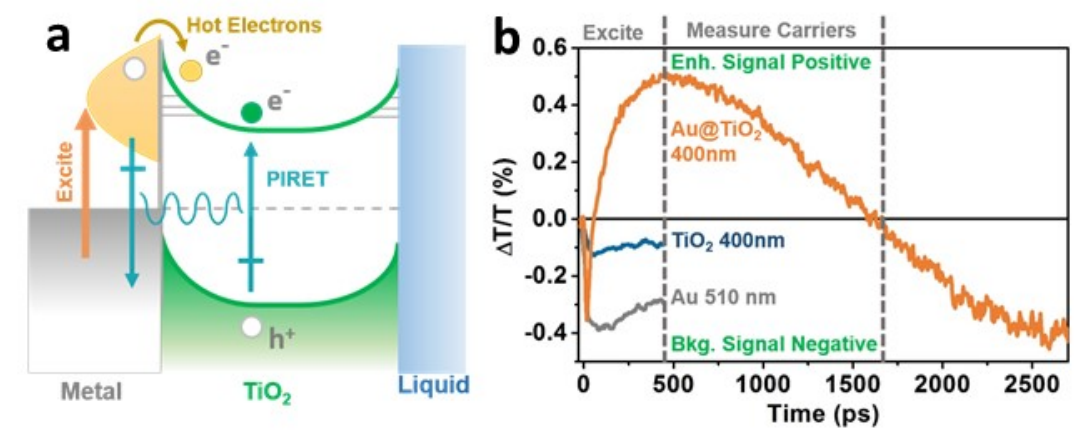

Figure 5.10. Determining plasmonic enhancement mechanism by transient absorption spectroscopy. a, After excitation of the plasmon by the pump laser the plasmon can enhance carrier creation in the $\mathrm{TiO}_{2}$ by hot electron injection or PIRET. b, The complex equilibration present in trap states after excitation allows separation of plasmonic enhancement from excitation of the semiconductor or metal alone.

Figure 5.10b also shows the transient response for exciting the plasmon and probing the $\mathrm{TiO}_{2}$ in $\mathrm{Au} @ \mathrm{TiO}_{2}$. The data for $400 \mathrm{~nm}$ excitation is shown, but the response was similar across the pump wavelength range tested as shown in Figure 5.11. Immediately following excitation, the transient absorption signal was negative, similar to the $\mathrm{TiO}_{2}$ or Au background signal. However, the signal quickly switched to positive, corresponding to less carriers being present in the trap states. On longer time scales, the signal recovered to an absorption. The switch in transient absorption sign after exciting the plasmon corresponds to the equilibration that existed in the trap states and the metal/semiconductor and metal/liquid interfaces. While complex in origin, this effect allowed for the magnitude of the added carrier density to easily be separated, since without plasmonic enhancement both $\mathrm{TiO}_{2}$ and $\mathrm{Au}$ alone showed only an increased absorption.

Therefore, based on the difference in transient signal, the plasmonic enhancement was determined by scanning the pump from $400 \mathrm{~nm}$ to $700 \mathrm{~nm}$ and recording the maximum bleach (positive signal) attained at each wavelength. The relative change in the bleach verse the excitation wavelength then corresponds to the relative change in plasmon-enhanced carrier creation, and can be compared to theoretical predictions to determine the mechanism. For hot electron transfer in small metal nanoparticles, the energy of the hot electron distribution is proportional to the plasmon's absorption, and the relative increase in carrier density should follow this trend. ${ }^{22}$ For PIRET in the ensemble averaged transient absorption measurements, the relative increase in charge carrier density should follow the spectral overlap between the plasmon and the semiconductor absorption. ${ }^{25}$ By comparing the maximum signal instead of the peak signal at a given time delay, the possible change in lifetime due to the metal/semiconductor interface is avoided, and the plasmon enhancement mechanisms can be isolated.

The data corresponding to this analysis is shown in Figure 5.12. The pump pulse had a fluence of $\sim 8$ $\mathrm{mJ} / \mathrm{cm}^{2}$ for each excitation wavelength used, but in order to ensure accurate relative transient signals for each core@shell nanoparticle, the signal was scaled by spot size and power. The un-processed data is shown in Figure 5.11. The maximum bleach was obtained by fitting the positive portion of each curve with a second order polynomial, with the error bars reported as the functional prediction intervals. This method was used instead of a simple average of the peak signal to reduce the effect of noise on the extracted enhancements. 

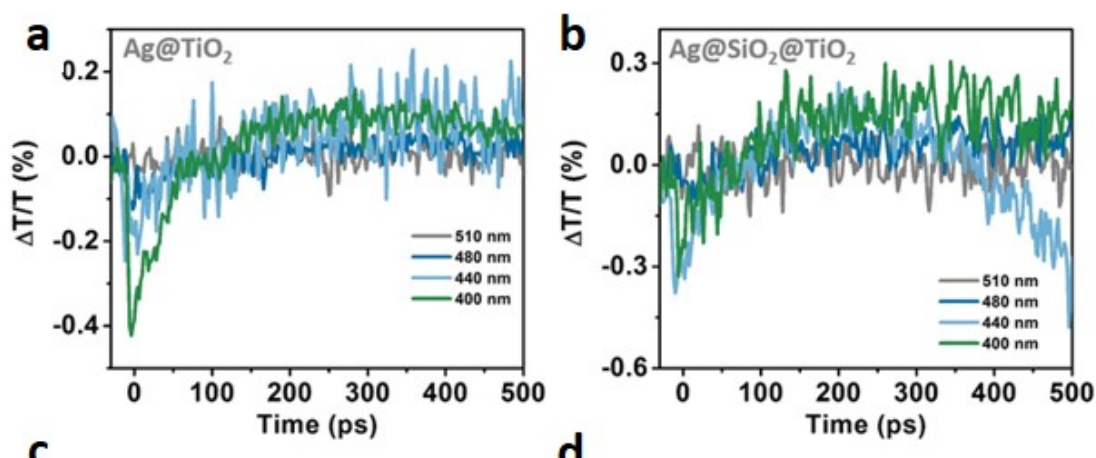

C

d
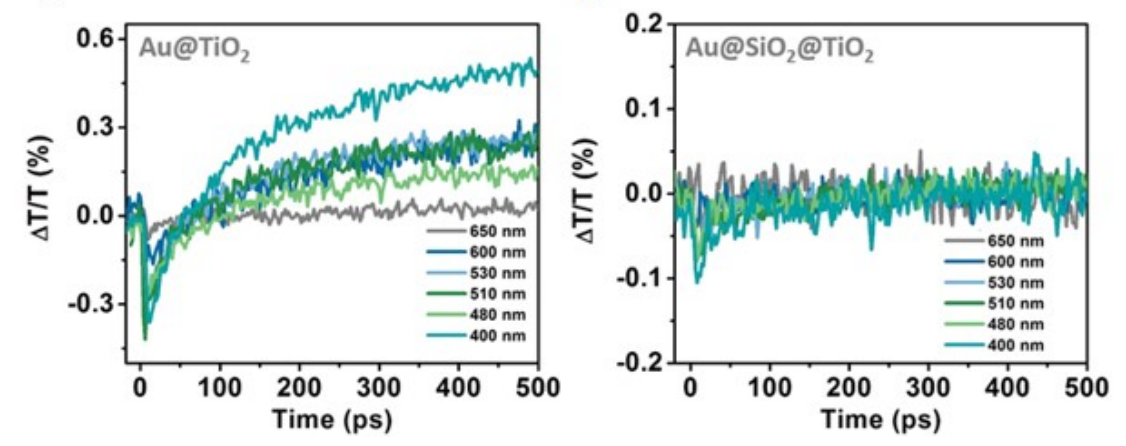

Figure 5.11. Transient absorption signals at different excitation (pump) wavelengths for a, $\mathrm{Ag} @ \mathrm{TiO}_{2}, \mathbf{b}$, Ag@SiO $\mathrm{STiO}_{2}, \mathbf{c}, \mathrm{Au} @ \mathrm{TiO}_{2}$, and d,Au@SiO $2 @ \mathrm{TiO}_{2}$.
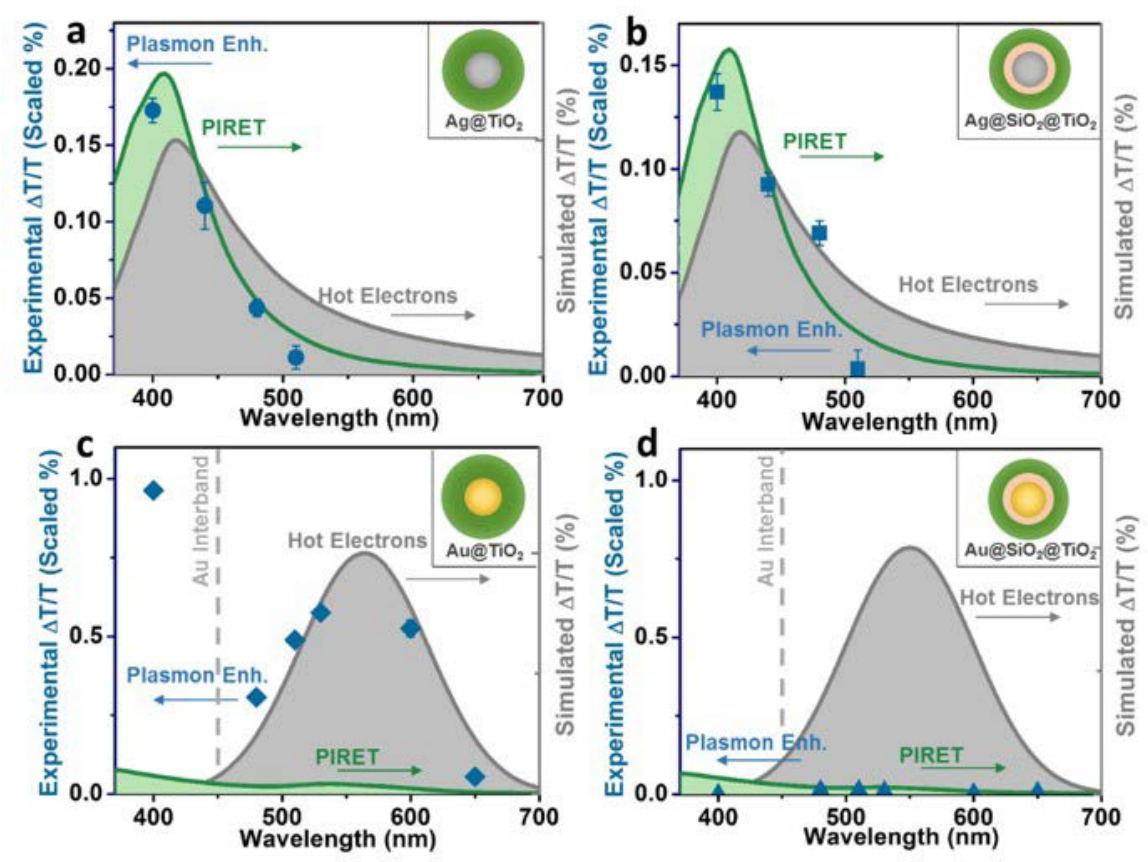

Figure 5.12. Control of plasmonic enhancement mechanism. Data points are taken from transient absorption measurements. The theoretical enhancement predicted for PIRET and hot electrons are shown as filled curves. a, In $\mathrm{Ag} @ \mathrm{TiO}_{2}$ contact and spectral overlap exists between metal and semiconductor, and PIRET and hot electron injection are both measured. b, Addition of a $\mathrm{SiO}_{2}$ barrier to Ag@ $\mathrm{TiO}_{2}$ eliminates hot electron injection. c, Switching the metal core to Au eliminates spectral overlap and PIRET. d, Inserting a $\mathrm{SiO}_{2}$ barrier in $\mathrm{Au} @ \mathrm{TiO}_{2}$ eliminates both hot electron injection and PIRET despite strong light absorption by plasmonic Au. The transient absorption percentage is scaled 
to correct for incident power fluctuations at each wavelength. The simulated data is fit to the experimental data in amplitude but not spectral dependence.

\subsubsection{Discussion}

In order to predict the possible enhancement from each mechanism, the absorption of the plasmon alone in $\mathrm{Ag} @ \mathrm{TiO}_{2}$ and $\mathrm{Ag} @ \mathrm{SiO}_{2} @ \mathrm{TiO}_{2}$ was extracted from the extinction by subtracting the $\mathrm{TiO}_{2}$ background. For Au@TiO ${ }_{2}$ and $\mathrm{Au} @ \mathrm{SiO}_{2} @ \mathrm{TiO}_{2}$, the $\mathrm{TiO}_{2}$ background was subtracted and a Gaussian fit was additionally used to separate the plasmon absorption from the interband transitions that occur at higher energies. After this procedure, the extracted absorption was taken as the possible enhancement from hot electrons, and the spectral overlap representing PIRET was obtained by multiplying the plasmon's extracted absorption by the $\mathrm{TiO}_{2}$ absorption. The theoretical predictions were then plotted with an amplitude that best fit the measured transient signal verse excitation wavelength in Figure 5.12. On each plot, the plasmon enhancement range is reported until further changing the pump wavelength no longer produced a measurable transient absorption signal above background. Further, the negative background signals in Figure 5.10 could only be measured by using larger concentration and pump fluences, ensuring the negative background signal did not detract from the positive plasmon enhanced signal in Figure 5.12. The disappearance of the positive transient signal at long wavelengths also confirmed that background contributions were not changing the measured enhancement.

The relative change in transmission $|\Delta T / T|$ in Figure 5.12 was directly proportional to the relative number of charge carriers created in $\mathrm{TiO}_{2}$ by plasmon-induced photoconversion. Theoretically, the hot electron injection and the PIRET processes can be distinguished by plotting $|\Delta T / T|$ as a function of the excitation wavelength. If hot electron injection dominates, the wavelength-dependent photoexcited carrier density in $\mathrm{TiO}_{2}$ will follow the lineshape of the plasmon absorption. ${ }^{22}$ If PIRET dominates, the wavelength-dependent photoexcited carrier density in $\mathrm{TiO}_{2}$ will depend on the spectral overlap. ${ }^{25}$ Figure 5.12a shows the resulting

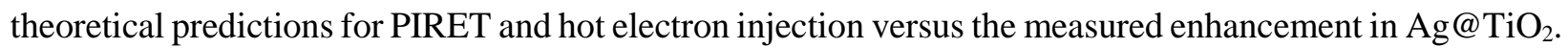
The measured enhancement, which corresponded to the scaled change in transient transmission, was observed to follow both the curve corresponding to PIRET and hot electrons, but weighted towards the spectral overlap representing PIRET. This suggested that both hot electron transfer and PIRET could be present but the overall enhancement was dominantly from PIRET, consistent with previous results for samples with large spectral overlaps. ${ }^{23-26}$ The enhancement extended to almost $500 \mathrm{~nm}$ before becoming negligible versus the noise of the measurement.

After insertion of an insulating $\mathrm{SiO}_{2}$ barrier in the $\mathrm{Ag@SiO}{ }_{2} @ \mathrm{TiO}_{2}$ sample (Figure 5.12b), similar

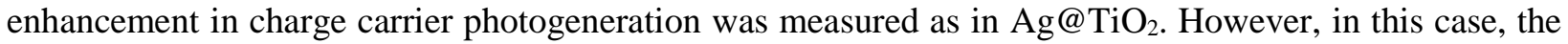
$\sim 10 \mathrm{~nm} \mathrm{SiO}_{2}$ barrier blocked hot electron transfer, making PIRET the only possible enhancement mechanism. Comparing Figure 5.12a with Figure 5.12b, the similarity in signal therefore confirmed that PIRET was dominant in Ag@ $\mathrm{TiO}_{2}$. This result was not surprising, given the lower possible maximum enhancement predicted for hot electrons in this geometry versus the strong enhancement known to be possible with non-radiative coupling. ${ }^{25.40}$

In $\mathrm{Au} @ \mathrm{TiO}_{2}$, the metal core was switched from $\mathrm{Ag}$ to $\mathrm{Au}$ and the $\mathrm{SiO}_{2}$ insulating barrier was removed. Since there was no spectral overlap between the SPR band of Au core and the absorption band of $\mathrm{TiO}_{2}$, PIRET was impossible. But the intimate contact between the Au core and the $\mathrm{TiO}_{2}$ shell made hot electron injection possible. This was confirmed in Figure 5.12c, wherein the relative transient absorption signal was observed to follow the plasmon's absorption band shape, consistent with theoretical prediction for hot electron transfer. ${ }^{22}$ The calculated spectral overlap was insignificant and weighted towards the $\mathrm{TiO}_{2}$ band 
edge, not describing the data. Interestingly, large photoconversion enhancement was found under excitation at $400 \mathrm{~nm}$. This energy corresponded to the interband transitions in Au, where it has been predicted that hot hole generation may be enhanced. ${ }^{22}$ A larger overall signal was measured for the $\mathrm{Au@} \mathrm{TiO}_{2}$ than Ag structures, as reflected in the smaller error bars relative to scaled transient absorption signal. This was indicative of the $\mathrm{Au} @ \mathrm{TiO}_{2}$ having trap states with a larger excited state absorption cross section for the 800 nm probe.

The presence of hot electron transfer in $\mathrm{Au@} \mathrm{TiO}_{2}$ was further confirmed by inserting the $\mathrm{SiO}_{2}$ spacer layer. Figure 5.12d shows that no plasmonic photoconversion enhancement was detected in $\mathrm{Au} @ \mathrm{SiO}_{2} @ \mathrm{TiO}_{2}$ above the background of the experiment, also seen in Figure 5.11. The lack of any enhancement mechanism was further verified by the negligible positive signal in the transients and similarity in signal with a thin and thick $\mathrm{SiO}_{2}$ barrier (Figure 5.13). In $\mathrm{Au} @ \mathrm{SiO}_{2} @ \mathrm{TiO}_{2}$, the silica spacer layer prevented hot electron transfer. The lack of spectral overlap between the metal and semiconductor disenabled the PIRET process. Hence no plasmonic enhancement was observed below the band edge of $\mathrm{TiO}_{2}$ although the Au core showed strong light absorption.

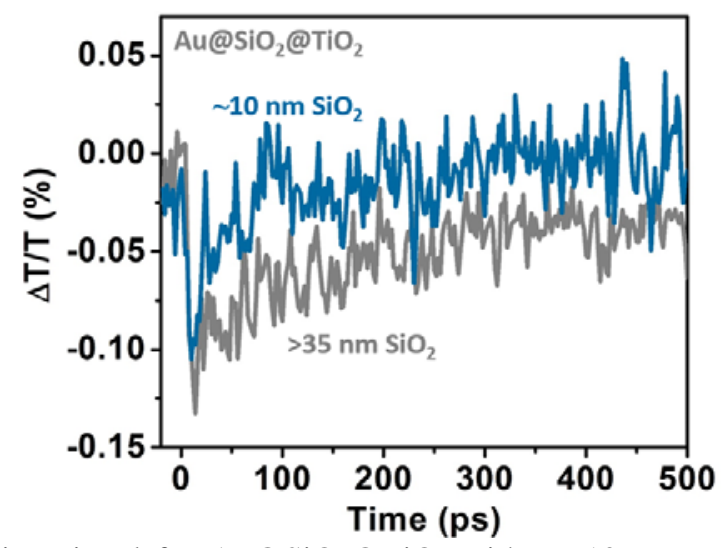

Figure 5.13. Transient absorption signal for $\mathrm{Au} @ \mathrm{SiO}_{2} @ \mathrm{TiO}_{2}$ with a $\sim 10 \mathrm{~nm}$ and $>35 \mathrm{~nm} \mathrm{SiO}_{2}$ insulator barrier thickness between metal and semiconductor. The pump wavelength is $400 \mathrm{~nm}$.

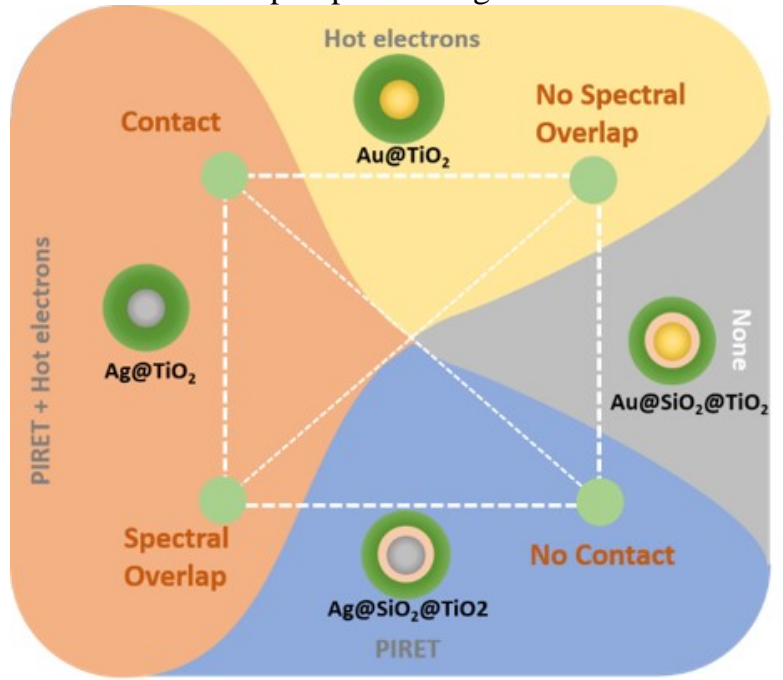

Figure 5.14. The variables that control the possible plasmonic enhancement mechanisms for extending photoconversion. Connecting the line between the properties of the given core@shell nanoparticle gives the corresponding enhancement mechanisms present. 
The metal@core shell nanoparticles confirmed that the plasmonic enhancement mechanism can be engineered by the metal-semiconductor spectral properties and relative proximity. The sample space covered is summarized in Figure 5.14. The chart can be used to quickly determine which enhancement mechanism will most likely be present in a given sample. For Ag@SiO $\mathrm{S}_{2} \mathrm{TiO}_{2}$, no physical contact was present but spectral overlap was. Connecting these points in Figure 5.14 predicts only PIRET is present, in agreement with Figure 5.12b. Similarly, for $\mathrm{Au@} \mathrm{TiO}_{2}$, only hot electron transfer was possible due to the lack of spectral overlap, and both enhancement mechanisms were present in Ag@ $\mathrm{TiO}_{2}$. If the spectral overlap and contact are both not present, the plasmon cannot extend the range over which photoconversion is possible.

\subsection{Chapter Summary}

In this Chapter, the three mechanisms discovered in Chapter 2-4 were related to the plasmon's dephasing time, allowing a unified outlook as well as the ability to use plasmonics in a predictive manner for maximum efficiency calculations. These calculations showed how the plasmon is best used in photovoltaics and photocatalysis. The predictions matched the results of Chapter 2-4 in showing that the plasmon's near field, whether used in light trapping or near-band edge enhancement, almost always leads to the largest enhancement. Hot carrier injection is promising, but more work must be done on optimizing injection beyond $10 \%$ if efficiency is to reach that of the near-field enhancements. It was also proven that plasmonics can restore a weakly absorbing semiconductor to thin film limits - allowing recombination and migration issues to be lessened or manufacturing constraints relaxed while still allowing high efficiency solar to fuel or solar to electrical conversion.

Towards achieving the guidelines given by these calculations, the parameters that control the presence of hot electron injection and PIRET were experimentally confirmed, setting up a design chart (Figure 5.14) to predict which mechanism is present for a given plasmonic architecture. While the plasmon's dephasing is critical to achieving high conversion efficiencies, it is still important to systematically design the semiconductor-metal system to avoid the deleterious effects brought up in Chapter 2-4.

\subsection{References}

1. Shockley, W.; Queisser, H. J. J. Appl. Phys. 1961, 32, 510.

2. Shah, A.; Torres, P.; Tscharner, R.; Wyrsch, N.; Keppner, H. Science 1999, 285, 692.

3. Bolton, J.; Strickler, S.; Connolly, J. Nature 1985, 316, 495.

4. Weber, M. F.; Dignam, M. J.; J. Electrochem. Soc. 1984, 131, 1258.

5. a De Vos, J. Phys. D: Appl. Phys. 1980, 13, 839.

6. Ross, R. T.; Hsiao, T. L. J. Appl. Phys. 1977, 48, 4783.

7. Ross, R. T.; Nozik, A. J. J. Appl. Phys., 1982, 53, 3813.

8. Nozik, A. J. Chem. Phys. Lett., 2008, 457, 3.

9. Trupke, T.; Green, M. A.; Würfel, P. J. Appl. Phys. 2002, 92, 4117.

10. Hanna, M. C.; Nozik, A. J. J. Appl. Phys. 2006, 100, 074510.

11. Willets, K. A.; Van Duyne, R. P. Annu. Rev. Phys. Chem. 2007, 58, 267.

12. Atwater, H. A.; Polman, A. Nature Mater. 2010, 9, 205.

13. Sonnichsen, C.; Franzl, T.; Wilk, T.; Plessen, G.; Feldmann, J.; Wilson, O.; Mulvaney, P. Phys. Rev. Lett. 2002, 88, 077402.

14. Catchpole, K. R.; Polman, A. Opt. Express 2008, 16, 21793. 
15. Aydin, K.; Ferry, V. E.; Briggs, R. M.; Atwater, H. A. Nature Commun. 2011, 2, 517.

16. Schuller, J. A.; Barnard, E. S.; Cai, W.; Jun, Y. C.; White, J. S.; Brongersma, M. L. Nature Mater. 2010, 9, 193.

17. Pala, R. A.; Liu, J. S. Q.; Barnard, E. S.; Askarov, D.; Garnett, E. C.; Fan, S.; Brongersma, M. L. Nature Commun. 2013, 4, 2095.

18. Ferry,V. E.; Verschuuren, M. A.; Li, H. B. T.; Verhagen, E.; Walters, R. J.; Schropp, R. E. I.; Atwater, H. A.; Polman, A. Opt. Express 2010, 18, 237.

19. Furube, A.; Du, L.; Hara, K.; Katoh, R.; Tachiya, M. J. Am. Chem. Soc. 2007, 129, 14852.

20. Brongersma, M. L.; Halas, N. J.; Nordlander, P. Nature Nanotechnol. 2015, 10, 25.

21. Mubeen, S.; Lee, J.; Singh, N.; Krämer, S.; Stucky, G. D.; Moskovits, M. Nature Nanotechnol. 2013, 8, 247.

22. Govorov, A. O.; Zhang, H.; Gun'ko, Y. K. J. Phys. Chem. C 2013, 117, 16616.

23. Li, J.; Cushing, S. K.; Zheng, P.; Senty, T. R.; Meng, F.; Bristow, A. D.; Manivannan, A.; Wu, N. J. Am. Chem. Soc. 2014, 136, 8438.

24. Cushing, S. K.; Li, J.; Meng, F.; Senty, T. R.; Suri, S.; Zhi, M.; Li, M.; Bristow, A. D.; Wu, N. Q. J. Am. Chem. Soc. 2012, 134, 15033.

25. Li, J.; Cushing, S. K.; Meng, F.; Senty, T. R.; Bristow, A. D.; Wu, N. Q. Nature Photonics 2015, 9, 601-607.

26. Cushing, S. K.; Li, J.; Bright, J.; Yost, B.; Zheng, P.; Bristow, A. D.; Wu, N. Q. J. Phys. Chem. C 2015, 119, 16239.

27. Thimsen, E.; Formal, F. L.; Grätzel, M.; Warren, S. C. Nano Lett. 2010, 11, 35.

28. Standridge, S. D.; Schatz, G. C.; Hupp, J. T. J. Am. Chem. Soc. 2009, 131, 8407.

29. Kelly, K. L.; Coronado, E.; Zhao, L. L.; Schatz, G. C. J. Phys. Chem. B 2003, 107, 668.

30. Campion, A.; Kambhampati, P. Chem. Soc. Rev. 1998, 27, 241.

31. Scaffardi, L. B.; Pellegri, N.; De Sanctis, O.; Tocho, O. J. Nanotechnology 2005, 16, 158.

32. Sadeghi, S. M. Phys. Rev. A 2013, 88, 013831.

33. Zhang, W.; Govorov. A. O.; Bryant, G. W. Phys. Rev. Lett. 2004, 97, 146804.

34. Artuso, R. D.; Bryant, G. W. Phys. Rev. B 2010, 82, 195419.

35. Cushing, S. K.; Bristow, A. D.; Wu, N. Physical Chem. Chem. Phys. 2015, DOI: 10.1039/C5CP04512F

36. Varada, G. V.; Agarwal, G. S. Phys. Rev. A 1992, 45, 6721.

37. Mandel, L.; Wolf, E. Optical Coherence and Quantum Optics; Cambridge University Press: New York, 1995.

38. Becker, P. C.; Fragnito, H. L.; Brito Cruz, C. H.; Fork, R. L.; Cunningham, J. E.; Henry, J. E.; Shank, C. V. Phys. Rev. Lett. 1988, 61, 1647.

39. John, S.; Soukoulis, C.; Cohen, M. H.; Economou,E. N. Phys. Rev. Lett. 1986, 57, 1777.

40. Leenheer, A. J.; Narang, P.; Lewis, N. S.; Atwater, H. A. J. Appl. Phys. 2014, 115, 134301.

41. Li, J.; Cushing, S. K.; Bright, J.; Meng, F.; Senty, T. R.; Zheng, P.; Bristow, A. D.; Wu, N. ACS Catal. 2013, 3, 47.

42. Meng, F.; Cushing, S. K.; Li, J.; Hao, S.; Wu, N. ACS Catal. 2015, 5, 1949.

43. Giugni, A.; Torre, B.; Toma, A.; Francardi, M.; Malerba, M.; Alabastri, A.; Zaccaria, R. P.; Stockman, M. I.; Di Fabrizio, E. Nature Nanotechnol. 2013, 8, 845.

44. Kim, S. S.; Na, S. I.; Jo, J.; Kim, D. Y.; Nah, Y. C. Appl. Phys. Lett. 2008, 93, 073307.

45. Tan, H.; Santbergen, R.; Smets, A. H. M.; Zeman, M. Nano Lett. 2012, 12, 4070. 
46. Liu, X.; Atwater, M.; Wang, J.; Huo, Q. Colloids Surf. B 2007, 58, 3.

47. Link, S.; El-Sayed, M. A. J. Phys. Chem. B 1999, 103, 8410.

48. Rosencher, E. Optoelectronics; Cambridge University Press: New York, 2002.

49. Shah, J. Ultrafast Spectroscopy of Semiconductors and Semiconductor Nanostructures; Springer: New York, 1999.

50. Peña-Rodríguez, O.; Pérez, P. P. G.; Pal, U. Int. J. Spectrosc. 2011, 583743, 1-10.

51. Scaffardi, L. B;.Pellegri, N.; de Sanctis, O.; Tocho, J. O. Nanotechnology 2005, 16, 158.

52. Seh, Z.; Liu, S.; Low, M.; Zhang, S.; Liu, Z.; Mlayah, A.; Han, M. Adv. Mater. 2012, 24, 23102314.

53. Lu, Y.; Yin, Y.; Li, Z.; Xia, Y. Nano Lett. 2002, 2, 785-788.

54. Yoshihara, T.; Katoh, R.; Furube, A. J. Phys. Chem. B 2004, 108, 3817-3823. 


\section{Chapter 6. Conclusion}

\subsection{Summary of Thesis}

In this thesis, the mechanisms of plasmon-enhanced solar energy harvesting have been isolated, including the discovery of a new near-field mediated enhancement, and the viability of plasmonics for efficient photovoltaics and photocatalysis has been explored. The plasmon's response was broken into scattering of incident light, by which multiple reflections can increase light absorption in the semiconductor at energies above the band gap; light absorption in the metal for hot carrier generation, for which subsequent transfer to the semiconductor can create excited carriers even at energies below the semiconductor's band gap; and PIRET, in which the plasmon's near field is used to non-radiatively excite interband transitions in the semiconductor both above and near the band edge. When light is incident on a metal nanoparticle, all three responses are present, but to differing degrees based on the balance of non-radiative to radiative dephasing processes, themselves determined by the metal nanoparticle size, shape, constituent metal, and metal-semiconductor interactions. The metal nanoparticle was also shown to trap and transport excited carriers, in a manner which can be advantageous or deleterious depending on metal nanoparticle location within the device.

This thesis has shown the complex response of the plasmon will not be adequately utilized by only matching the plasmon resonance to where more light needs to be absorbed and integrating metal nanoparticles with the semiconductor, explaining the many low peak efficiencies in plasmon-enhanced solar energy conversion to date. Instead, the proper plasmonic enhancement mechanism must be selected to compensate a given semiconductors weaknesses, whether in absorption, spectral range, or lifetimes. Given the near field, scattering, and hot electron response of the plasmon is linked by the dephasing time, one mechanism or all three can be used. However it is easiest to design a structure to isolate one effect. Even before the link between dephasing and plasmonic response was known, this strategy allowed a high performance solar water splitting architecture to be created for each of the three enhancements in Chapters 2-4. As seen in Section 4.3, however, ignoring dephasing was still decreasing performance by up to $50 \%$. Given the already high efficiency achieved compared to stand-alone semiconductor designs of similar materials, this gives great promise for plasmonic enhancement, but only if the following dephasing-based design guidelines from Chapter 5 are used:

Hot electrons were predicted best utilized for large band gap semiconductors, selecting the plasmonic structure to produce the largest hot carrier yield. This includes balancing spectral position and geometry to achieve the optimal dephasing, as well as considering the energy of the created hot carriers. The metalsemiconductor geometry should also be constructed to prevent charge equilibration problems. Scattering is best used above the band gap of semiconductors with ideal band gaps, allowing thinner films which decrease recombination losses while maintaining absorptions strengths. The plasmonic structure should allow maximum reflection and minimal absorption. PIRET was predicted to create the largest overall enhancement, and is best used with a thin barrier between the metal and semiconductor to prevent interfacial dephasing. PIRET should be used with non-radiative semiconductors to prevent back-transfer by FRET, and can lead to large increases below the band gap where the semiconductor absorbs weakly. In particular, PIRET can extend the photoconversion range with efficiencies similar to above-band edge, making it promising for combatting large band gaps. Regardless of the chosen enhancement mechanism, the geometry must be constructured so that the plasmon is encased in the semiconductor, but still near enough to the active area to promote charge seperation where needed spatially. 


\subsection{Future Research}

While plasmonics has a remarkable ability to control and manipulate light, it must be remembered that the plasmon is not the solar cell, rather a patch to compensate a given semiconductor's weaknesses. Whether scattering, hot electrons, or PIRET, just because the plasmon strongly absorbs light does not guarantee the energy will be transferred to the semiconductor. In order to use plasmonics effectively, the appropriate enhancement mechanism must be selected, such as hot carrier transfer for wide band gaps, scattering for weak absorption coefficients, or PIRET for both. The spectral needs must be balanced against recombination, both in considering the metal-semiconductor interface and overall device geometry. This leads to a large set of parameters to optimize for a given plasmonic structure, and the complex metalsemiconductor interactions cannot be optimized simply by tuning the plasmon's resonance and local field.

To simplify design, the parameters controlling the plasmonic enhancement can be distilled to the dephasing time. The dephasing time can be calculated using the same FDTD simulations already common in planning plasmonic solar cells through the predicted plasmon linewidth $(\Gamma)$ as $T_{2}=2 \hbar / \Gamma$, where $2 \hbar=1316 \mathrm{meV} \cdot \mathrm{fs}$, giving a central criteria that is easily predicted and optimized. On this basis, and selecting the best enhancement for the needs of the semiconductor, the plasmonic geometry can be further refined to optimize hot carrier transfer, near-field coupling, or light trapping. Remembering that a solar cell already operating near the Shockley-Queisser limit cannot be greatly enhanced by plasmonics, as the plasmon only increases photoconversion in the semiconductor, plasmonics still has great promise to allow cheap to construct materials to match single crystalline performance levels, ushering in a renewable energy economy. 


\section{Appendix: Publication Record}

(two bolded names and * indicates shared first authorship)

1. Investigation of Band Gap Narrowing in Nitrogen-Doped $\mathrm{La}_{2} \mathrm{Ti}_{2} \mathrm{O}_{7}$ with Transient Absorption Spectroscopy,

B. T. Yost, S. K. Cushing, F. Meng, J. Bright, D. A. Bas, N. Wu, A. D. Bristow, Physical Chemistry Chemical Physics (2015), DOI: 10.1039/c5cp05637c

2. Maximum Efficiency Calculations for Plasmonic Enhancement of Solar Energy Conversion by Scattering, Hot Electrons, and the Near Field,

S. K. Cushing, A. D. Bristow, N. Wu, Physical Chemistry Chemical Physics (2015), DOI: 10.1039/C5CP04512F

3. Harvesting Sunlight below the Semiconductor Band-Edge by Plasmonic Energy Transfer, J. Li,* S. K. Cushing, * F. Meng, T. R. Senty, A. D. Bristow, N. Wu, Nature Photonics, 9 (2015), 601-607.

4. Controlling Plasmon-Induced Resonance Energy Transfer and Hot Electron Injection Processes in Metal@TiO ${ }_{2}$ Core-Shell Nanoparticles,

S. K. Cushing, J. Li, J. Bright, B. Yost, P. Zheng, A. D. Bristow, N. Wu, Journal Physical Chemistry C, 119 (2015), 16239-16244.

5. A Surface-Enhanced Raman Scattering Sensor Integrated with Battery-Controlled Fluidic Device for Capture and Detection of Trace Small Molecules Q. Zhou, G. Meng, P. Zheng, S. K. Cushing, N. Wu, Q. Huang, C. Zhu, Z. Zhang, Z. Wang, Scientific Reports, 5 (2015), 1-10.

6. A Gold Nanohole Array Based Surface-Enhanced Raman Scattering (SERS) Biosensor for Detection of $\mathrm{Ag}(\mathrm{I})$ and Mercury(II) in Human Saliva,

P. Zheng, M. Li, R. Jurevic, S. K. Cushing, Y. Liu, N. Wu, Nanoscale, 7 (2015), 11005-11012.

7. Inverting Transient Absorption Data to Determine Transfer Rates in Quantum Dot- $\mathrm{TiO}_{2}$ Heterostructures,

T. R. Senty,* S. K. Cushing, * C. Wang, C. Matranga, A. D. Bristow, Journal of Physical Chemistry C, 119 (2015), 6337-6343.

8. Enhancement of Solar Hydrogen Generation by Synergistic Interaction of $\mathrm{La}_{2} \mathrm{Ti}_{2} \mathrm{O}_{7}$ Photocatalyst with Plasmonic Gold Nanoparticles and Reduced Graphene Oxide Nanosheets, F. Meng, S. K. Cushing, J. Li, S. Hao, N. Wu, ACS Catalysis, 5 (2015), 1949-1955.

9. Band gap narrowing in nitrogen-doped $\mathrm{La}_{2} \mathrm{Ti}_{2} \mathrm{O}_{7}$ predicated by density-functional theory calculation, J. Zhang, W. Dang, Z. Ao, S. K. Cushing, N. Q. Wu, Physical Chemistry Chemical Physics, 17 
(2015), 8994-9000.

10. Tailoring plasmonic properties of gold nanohole arrays for surface-enhanced Raman scattering, P. Zheng, S. K. Cushing, S. Suri, N. Wu, Physical Chemistry Chemical Physics, (2015), DOI: 10.1039/С4CP05291A

11. Above and Below Band Edge Light Recovery with Plasmonics, S. K. Cushing, J. Li, A. D. Bristow, N. Q. Wu, SPIE OPTO, 9358 (2015), 935811.

12. Plasmon-enhanced Optical Sensors: A Review, M. Li, S. K. Cushing, N. Q. Wu, Analyst, 140 (2015), 386-406.

13. Solar Hydrogen Generation by a CdS-Au-TiO 2 Sandwich Nanorod Array Enhanced with Au Nanoparticle as Electron Relay and Plasmonic Photosensitizer, J. Li,* S. K. Cushing, * P. Zheng, T. Senty, F. Meng, A. D. Bristow, A. Manivannan, N. Q. Wu, Journal of the American Chemical Society, 136 (2014), 8438-8449.

14. Origin of Strong Excitation Wavelength Dependent Fluorescence of Graphene Oxide, S. K. Cushing, J. Li, F. Huang, N. Q. Wu, ACS Nano, 8 (2014), 1002-1013.

15. Photoluminescence Spectroscopy Of $\mathrm{YVO}_{4}: \mathrm{Eu}^{3+}$ Nanoparticles with Aromatic Linker Molecules: A Precursor to Biomedical Functionalization, T. R. Senty, M. Yalamanchi, Y. Zhang, S. K. Cushing, M. S. Seehra, X. Shi, A. D. Bristow, Journal of Applied Physics, 115 (2014), 163107.

16. Plasmon-induced Photonic and Energy Transfer Enhancement of Solar Water Splitting by a Hematite Nanorod Array,

J. Li,* S. K. Cushing,* P. Zheng, F. Meng, D. Chu, N. Q. Wu, Nature Communications, 4 (2013), 2651.

17. Enhancing Charge Separation in Semiconductors through Plasmon Induced Resonant Energy Transfer,

S. K. Cushing and N. Q. Wu, Interface, 22 (2013), 63-67.

18. Asymmetric Silver “Nanocarrot” Structures: Solution Synthesis and their Asymmetric Plasmonic Resonances, H. Liang, D. Rossouw, H. Zhao, S. K. Cushing, H. Shi, A. Korinek, H. Xu, F. Rosei, W. Wang, N. Q. Wu, G. A. Botton, D. Ma, Journal of the American Chemical Society, 135 (2013), 9616-9619.

19. Three-dimensional Hierarchical Plasmonic Nano-Architecture Enhanced Surface-Enhanced Raman Scattering Immuno-Sensor for Cancer Biomarker Detection in Blood Plasma, M. Li, S. K. Cushing, J. Zhang, S. Suri, R. Evans, W. P. Petros, L. F. Gibson, D. Ma, Y. Liu, and N. Q. Wu, ACS Nano, 7 (2013), 4967-4976. 
20. Solar Hydrogen Generation by Nanoscale P-N Junction of P-Type Molybdenum Disulfide/NType Nitrogen-Doped Reduced Graphene Oxide,

F. Meng, J. Li, S. K. Cushing, M. Zhi, N. Q. Wu, Journal of the American Chemical Society, 135 (2013), 10286-10289.

21. Photocatalytic Hydrogen Generation Enhanced by Band Gap Narrowing and Improved Charge Carrier Mobility in $\mathrm{AgTaO}_{3}$ By Compensated Co-Doping, M. Li, J. Zhang, W. Dang, S. K. Cushing, D. Guo, N. Q. Wu, P. Yin, Physical Chemistry Chemical Physics, 15 (2013), 16220-16226.

22. Photocatalytic Water Oxidation by Hematite/Reduced Graphene Oxide Composites, F. Meng, J. Li, S. K. Cushing, J. Bright, M. Zhi, J. Rowley, Z. Hong, A. Manivannan, A. D. Bristow, N. Q. Wu, ACS Catalysis, 3 (2013), 746.

23. Ag@ $\mathrm{Cu}_{2} \mathrm{O}$ Core-shell Nanoparticles as Visible-Light Plasmonic Photocatalysts, J. Li,* S. K. Cushing,* J. Bright, F. Meng, T. R. Senty, P. Zheng, A. D. Bristow, N. Q. Wu, ACS Catalysis, 3(2013), 47-51

24. Photocatalytic Activity Enhanced by Plasmonic Resonant Energy Transfer from Metal To Semiconductor, S. K. Cushing, J. Li, F. Meng, T. R. Senty, S. Suri, M. Zhi, M. Li, A. D. Bristow, N. Q. Wu, Journal of the American Chemical Society, 134 (2012), 15033-15041.

25. Plasmonic Nanorice Antenna on Triangle Nano-Array for Surface-Enhanced Raman Scattering Detection of Hepatitis B Virus DNA, M. Li, S. K. Cushing, H. Liang, S. Suri, D. Ma, N. Q. Wu, Analytical Chemistry, 85 (2013), 2072.

26. Shape-dependent Surface-enhanced Raman Scattering in Gold-Raman-Probe-Silica Sandwiched Nanoparticles for Biocompatible Applications,

M. Li, S. K. Cushing, J. Zhang, J. Lankford, Z. P. Aguilar, D. Ma, N. Q. Wu, Nanotechnology, 23 (2012), 115501.

27. Fingerprinting Photoluminescence of Functional Groups in Graphene Oxide, M. Li, S. K. Cushing, X. Zhou, S. Guo and N. Q. Wu, Journal of Materials Chemistry, 22 (2012), 23374-23379.

28. Size-dependent Energy Transfer between CdSe/ZnS Quantum Dots and Gold Nanoparticles, M. Li, S. K. Cushing, Q. Wang, X. Shi, L. A. Hornak, Z. Hong, N. Q. Wu, The Journal of Physical Chemistry Letters, 2 (2011), 2125-2129.

29. Origin of Localized Surface Plasmon Resonances in Thin Silver Film over Nanosphere Patterns, S. K. Cushing, L. A. Hornak, J. Lankford, Y. Liu, N.Q. Wu, Applied Physics A, 103 (2011), 955958. 
30. Electrodeposition of Poly(phenylene oxide) Nanoscale Patterns with Nanosphere Lithography, J. Kang, H. Li, S. K. Cushing, J. Wang, N. Wu, ECS Trans., 19 (2009) 159. 